\title{
A preliminary catalogue of Hikurangi, New Zealand, Slow Slip Earthquakes, from January 2000 to February 2014
}

\author{
B. Peter Baxter \\ A thesis submitted to Victoria University of Wellington in fulfillment of \\ requirements for the degree of Master of Science
}

School of Geography, Environment and Earth Science

Victoria University of Wellington

March 2015 



\section{Contents}

Contents .. $\mathrm{i}$

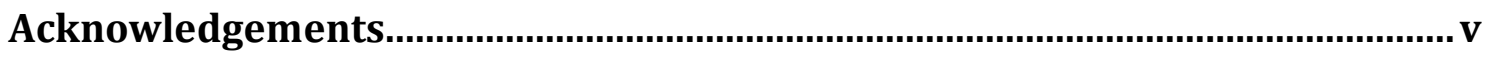

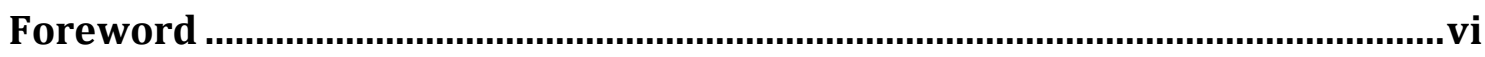

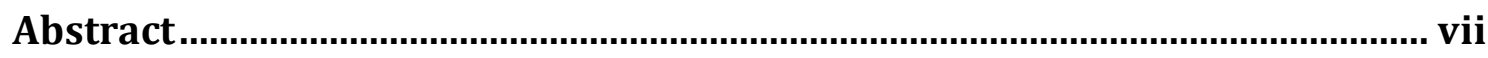

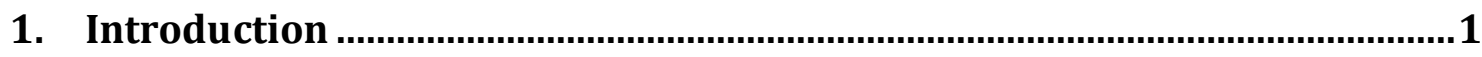

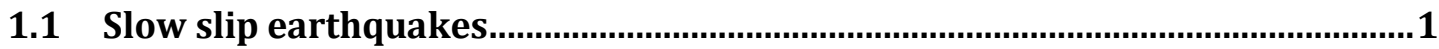

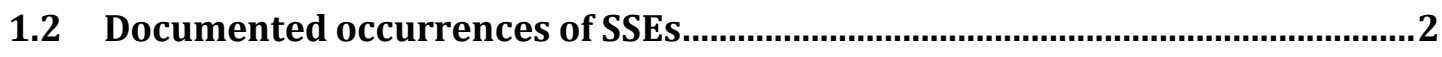

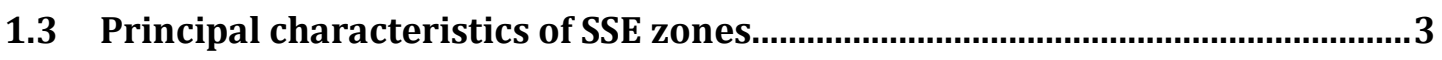

1.4 Tectonic setting of the Hikurangi margin ...............................................................5

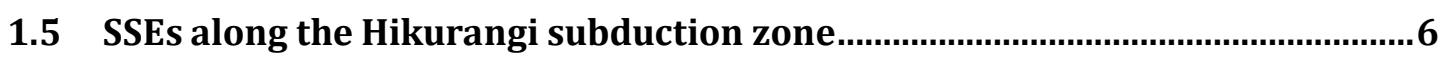

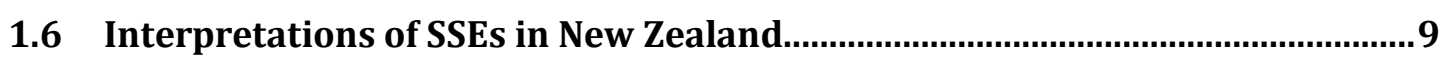

1.7 Thrust earthquakes on the Hikurangi subduction interface.......................... 13

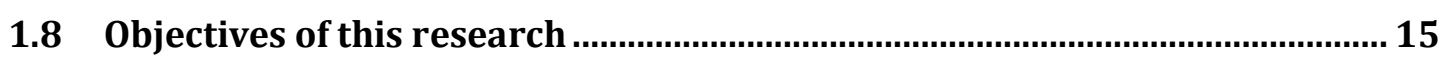

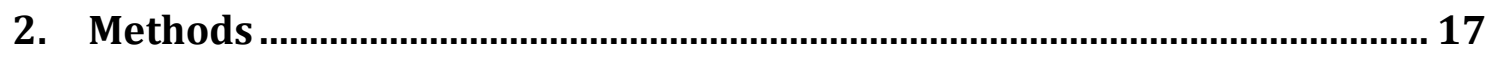

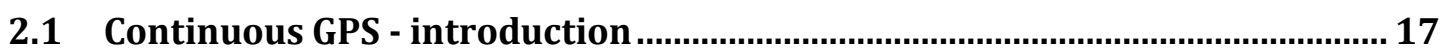

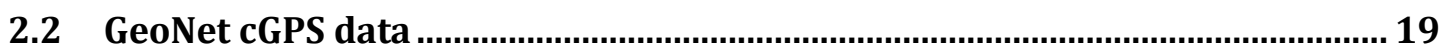

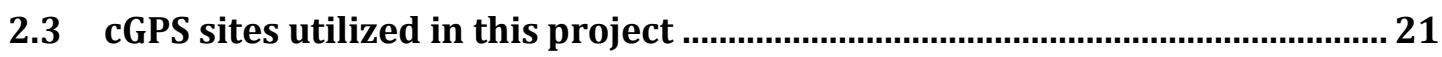

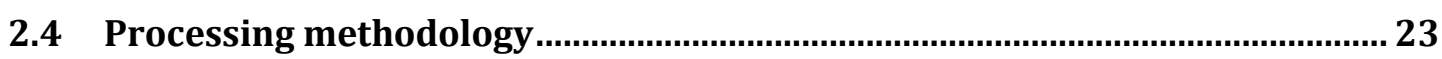

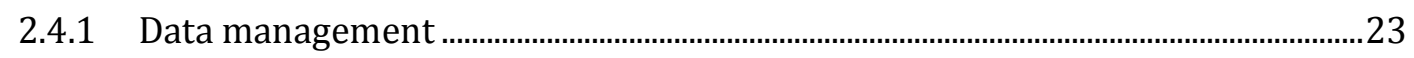

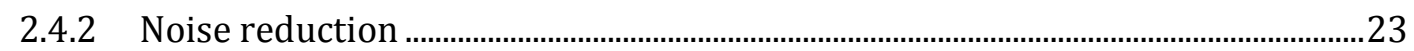

2.4.3 SSE identification and picking .....................................................................................

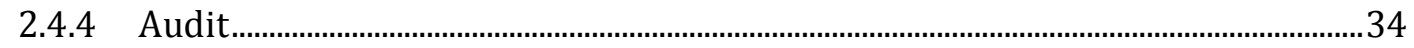

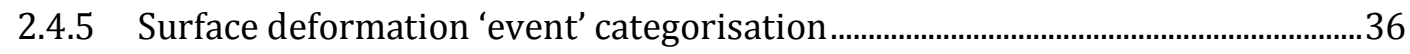

2.5 Temporal evolution: deformation-time shape analysis.................................36

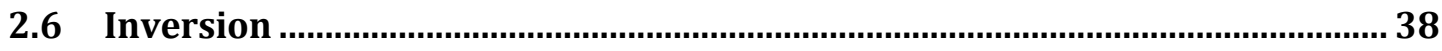

2.6.1 The Hikurangi subduction interface model .....................................................................38

2.6.2 Elastic response to source displacements - Inversion ............................................... 40

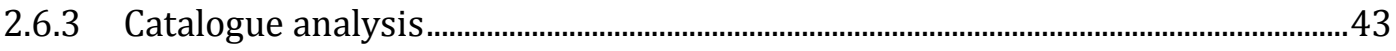

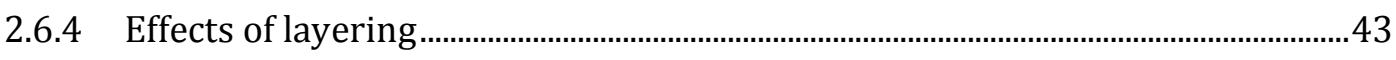

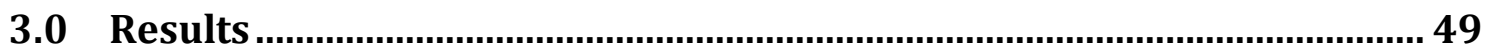




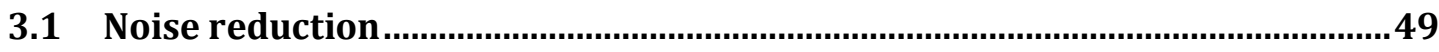

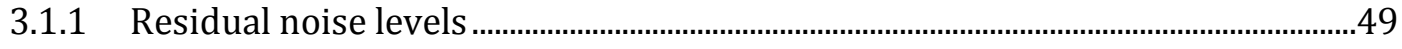

3.1.2 Factors affecting residual noise …………............................................................... 51

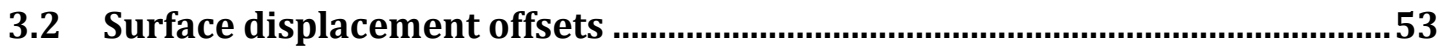

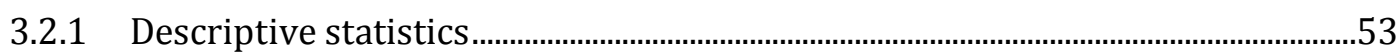

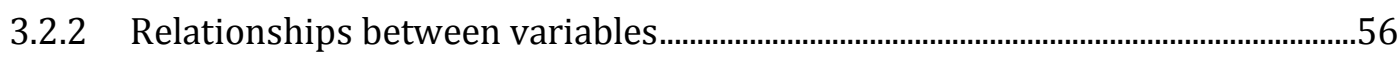

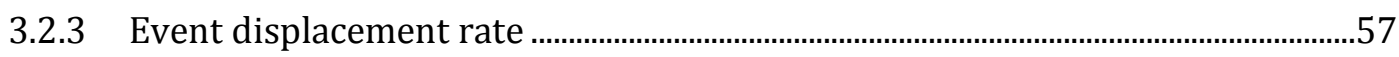

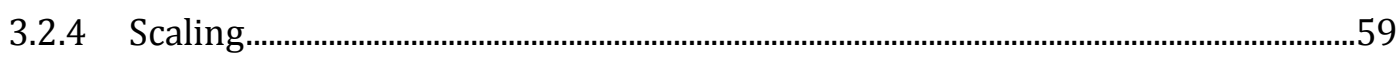

3.2.5 Temporal evolution: shape of surface displacement-time curves ........................61

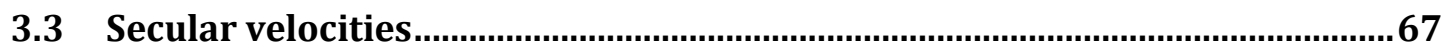

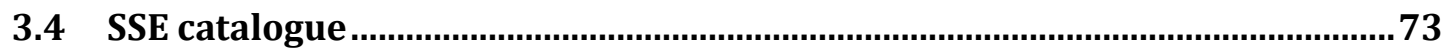

4.0 Effects of layering

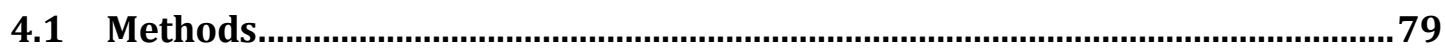

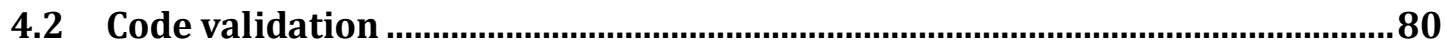

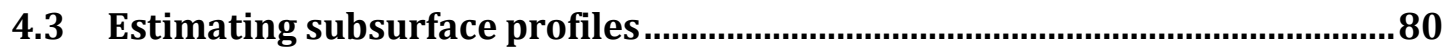

4.4 Tests on models of the Hikurangi subduction interface .....................................83

4.5 Systematic parametric study of the effects of layering .....................................85

4.6 Proposal for $1^{\text {st }}$ order correction to the Okada model .........................................86

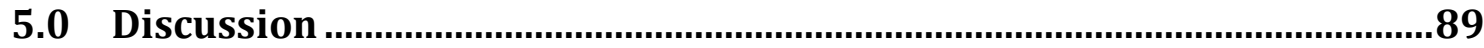

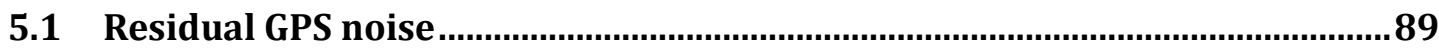

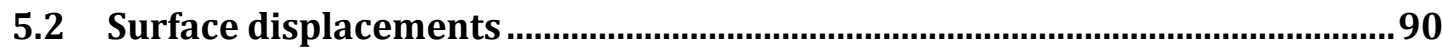

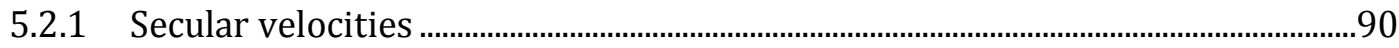

5.2.2 Displacements due to SSEs or other discrete events ...............................................95

5.2.3 'Negative' short-term displacements ……………………………………………….....95

5.3 Event displacement-time shapes (temporal evolution) .....................................97

5.3.1 Theoretical and observational background .................................................................98

5.3.2 Improving detection................................................................................................104

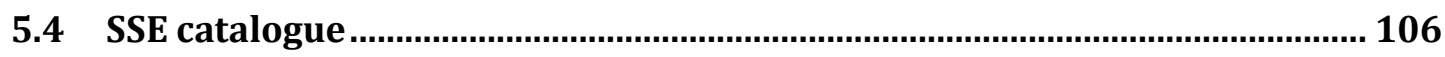

5.4.1 Comparison with published results ...................................................................106

5.4.2 Statistical analyses .............................................................................................107

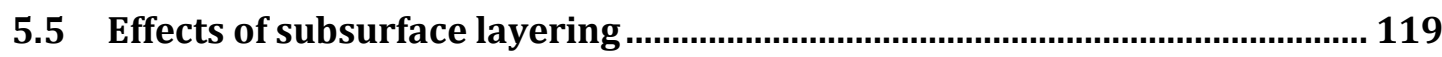

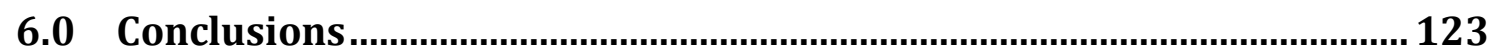

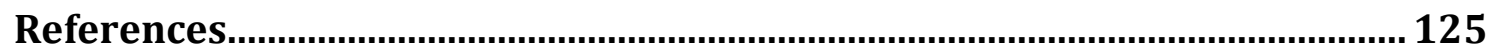


Appendix 1 - Geological legend for Fig. 2.3 ................................................ 143

Appendix 2 - Noise processing schema (ref. 2.4.2).......................................144

Appendix 3 - Spectral components of the regional stack ................................145

Appendix 4 - Jeffreys' Weighting and outlier treatment.................................146

Appendix 5 - Inversion methods used for SSEs in other subduction zones (ref.

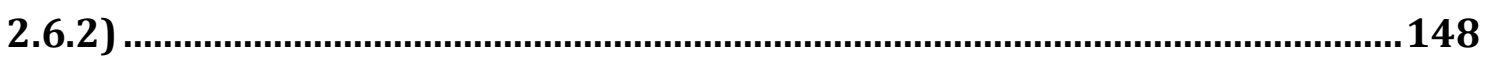

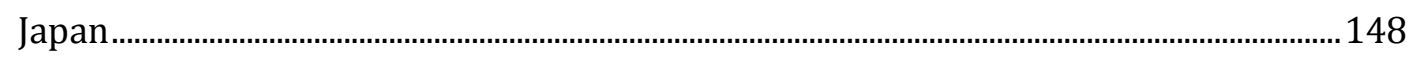

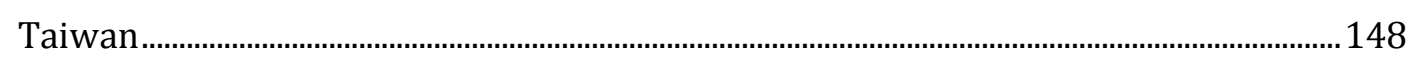

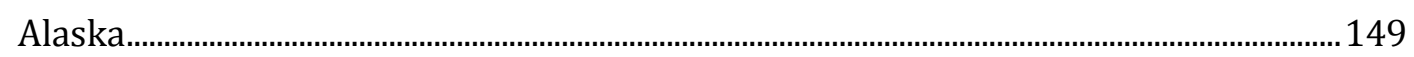

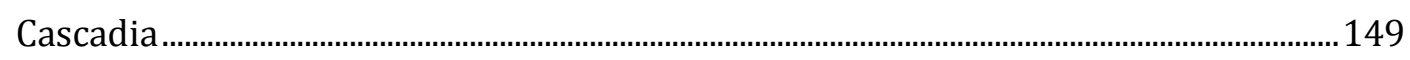

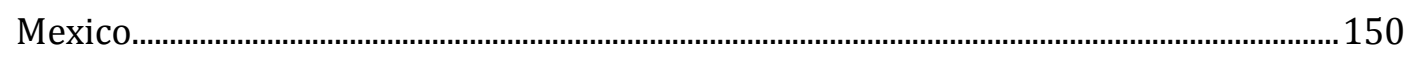

Ecuador, Peru and Chile ........................................................................................................ 150

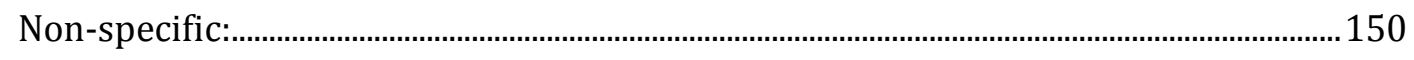

Appendix 6 - Comparison of interface models (ref. 2.6.1)............................151

Appendix 7 - Validation of Matlab implementation of Okada code, and EDGRN/EDCMP code (ref. 2.6.2) ......................................................................... 153

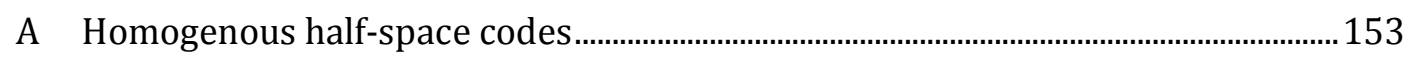

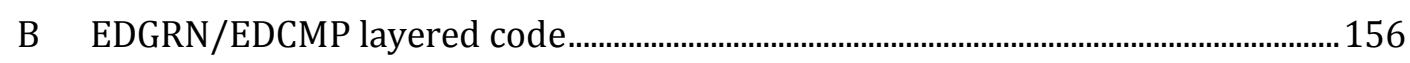

Appendix 8 - Factors affecting cGPS residual noise ......................................161

Appendix 9 - Evaluation of surface deformation event categorization (ref.

3.2.1)

Appendix 10 - Example of Tablecurve 2D fit to stacked normalized negative event 13 (ref. 3.2 .5 ) ......................................................................................... 167

Appendix 11 - Table of secular velocities (ref. 3.3)....................................168

Appendix 12 - Regional strain fields from secular velocity data (ref. 3.3) .. 172 Appendix 13 - Parametric study of the effects of layering ..............................182

Part 1: Shallow thrust fault (NE of South Island)...................................................183

Part 2: Deep strike-slip fault (SW of North Island) ..............................................187

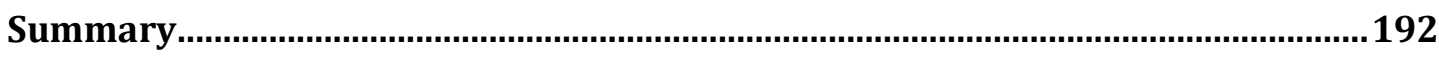


Appendix 14 - Modeling 'negative' surface displacements (ref. 5.2.3)......... 195

A. Subduction thrusts........................................................................................................................195

B. Imbricate faults and back-thrusts ............................................................................................198

Appendix 15 - Comparison of inversion results with Table 2 of Wallace and

Beavan (2010) (ref 5.4.1)...............................................................................199

Appendix 16 - Comparison of displacements at sites WGTN and WGTT...... 201

Note that figures and tables are numbered with the section in which they are first referenced. 


\section{Acknowledgements}

Firstly I must gratefully acknowledge financial support from the Frank Evison Research Scholarship and the EQC.

The New Zealand GeoNet project and its sponsors, EQC, GNS Science, and LINZ provided the GPS data used in this study.

Professor Euan Smith provided the inspiration for the study, and developed, and when requested, cheerfully modified most of the MATLAB code used extensively in this project. He has been a continual source of wisdom and encouragement throughout. Without his supervision and guidance I would have wandered off on too many sidetracks that attracted my curiosity.

Rafael Benites was responsible for re-awakening my long-subdued interest in all matters geotechnical and geophysical, and provided long hours of useful discussion over a number of years prior to my returning to university, and at odd times during this project.

Rachel Barrett was responsible for preliminary work on the cGPS processing for this project and sunnily inducted me into the ways of 'driving' Prof. Smith's code.

I would also like to thank the two examiners of this thesis, A/Prof. Lamb and A/Prof Lowry, for many useful comments that have allowed me to improve the manuscript.

Finally I should acknowledge the deep well of patience and support of my wife Mercedes, without which I would never have got to the end. 


\section{Foreword}

Most of the data processing and routine analysis for this project has utilized code developed by Prof. E. Smith using MATLAB [Mathworks, 2014].

Ancillary data manipulation has frequently been carried out using various versions of EXCEL [Microsoft, 2013].

Statistical analyses have usually been carried out using either Excel (for basic analyses), STATISTICA [StatSoft, 2013], or occasionally UNISTAT [Unistat, 2014]. Where Statistica graphical output has been utilized in figures, which are readily identified by the pale yellow border area, they may include the following default text in the title area: the type of plot with datasheet column headers, the name of the spreadsheet and number of variables (v) and cases (c), any inclusion or exclusion conditions, any regression or distribution parameter estimates where the plot includes a regression or distribution function, together with the type of confidence interval, or mean and standard deviation in the case of a distribution. In all cases the figure caption identifies the salient information and these default annotations may be ignored.

Curve fitting for analyses of event temporal evolution was carried out using Tablecurve 2D [Systat, 2012]. Where output has been used as illustrative figures (distinguished by purple border and black background), there are also a number of default statistical annotations that can be ignored.

Tests on inversion procedures have been carried out using source software identified in the text, usually running under GNU-FORTRAN [Approximatrix, 2014].

Some maps have been produced with the assistance of GoogleEarth [Google, 2013] and any $3^{\text {rd }}$ party overlays or templates have been identified. GE Graph [Sgrillo, 2014] has been utilized to produce graphical overlays in a number of cases.

Frederick W. Vollmer's software, Orient, was used for the production of figure 5.4.2.2c.

The term 'rms' is used frequently for the root mean squares of residuals from regression - the units should be clear from the context in each case. With the exception of Appendix 11, capitalized 'RMS' is reserved for the root mean squares of combined orthogonal displacement residuals arising from inversions. 


\section{Abstract}

This thesis documents processing carried out on cGPS data from 115 sites in the North Island and the top of the South Island of New Zealand in order to produce a catalogue of slow slip events (SSEs) for the Hikurangi Margin covering the period Jan 2000 to Feb 2014. It covers the background to the concept of SSEs and the reporting to date on their occurrence along the Margin, the methods used in the processing and analysis, the results of each significant step, and discussion of the results.

It has been shown that the processing route adopted in this work has reduced the average noise levels in the cGPS data by up to $67 \%$, and has eliminated virtually all correlated ("pink") noise, thus enabling the detection of small-amplitude events $(\sim 2 \mathrm{~mm}$ in cGPS signals).

One hundred and fifty events are catalogued in total, of which 137 are considered likely to be SSEs or similar. The catalogue includes estimates of the uncertainty in each parameter and is thus considered the most comprehensive to date. Sixteen of the inversion results were able to be directly compared with published information and showed satisfactory agreement on location and equivalent moment magnitudes.

The important aspects of the project that have been developed further than has been documented to date in the literature include: partitioning of the secular velocity field over the margin to allow the underlying tectonic signal to be better understood; detailed characterization of the temporal evolution of the SSEs; the identification of approximately 40 events that show slips in the opposite direction to that expected; and some preliminary conclusions concerning event scaling.

One of the objectives of the project was to identify whether there were fundamental differences in the characteristics of SSEs in the northeast and southwest of the margin. On the basis of the analyses to date, it appears that the events form a continuum, at least in terms of depth, temporal evolution, source slip rates and scaling, but in general terms the events in the southwest have been confirmed to be of longer duration than those in the northeast.

The project has identified further work that needs to be carried out or is ongoing in order to maximize the value of these new results. 



\section{Introduction}

\subsection{Slow slip earthquakes}

At a subduction zone, where the plate interface is 'locked', the down-going slab "drags" the overlying slab with it, producing a displacement field above the interface that approximately parallels the convergence direction. This strain accumulates during the inter-seismic period, and when the failure strength of some segment of the interface is exceeded, some of the strain is recovered by backward slip on the interface. In the case of a conventional earthquake this recovery takes place in a matter of seconds. The cycle then begins again. Slip can, however, take place on parts of the interface over a much longer period, lasting from hours to months, and in this case there is no strong-motion seismic radiation (and none of the consequent destruction). These slow slips can, however, release similar or even greater amounts of strain to conventional earthquakes, and therefore may play a significant role in strain-relief.

Detailed observations of Slow Slip Events (SSE), or slow earthquakes, have only been documented over the last two decades. Initially, most of these SSEs were detected by observations of anomalous geodetic trends in continuous Global Positioning System (cGPS) data [Douglas et al., 2005; McGuire and Segall, 2003; Melbourne, 2002], or using high-sensitivity borehole strainmeters [Sacks et al., 1978; 1982]. Fig. 1.1 illustrates typical signals in de-trended noise-reduced cGPS records indicative of at least three SSE events on the Hikurangi margin, one of which (occurring in 2007-08) is targeted in this plot.

Subsequently, associations with other seismic phenomena such as tremor have been used to extend catalogues of slow slip events [Bartlow et al., 2014; Holtkamp and Brudzinski, 2011; Ide et al., 2008; Obara, 2002; Peng and Gomberg, 2010; Schwartz and Rokosky, 2007; Wech, 2010; Wech et al., 2012]. However, recent work [Vidale et al., 2011; Wech and Bartlow, 2014] has thrown some doubt on the reliability of this proxy. 


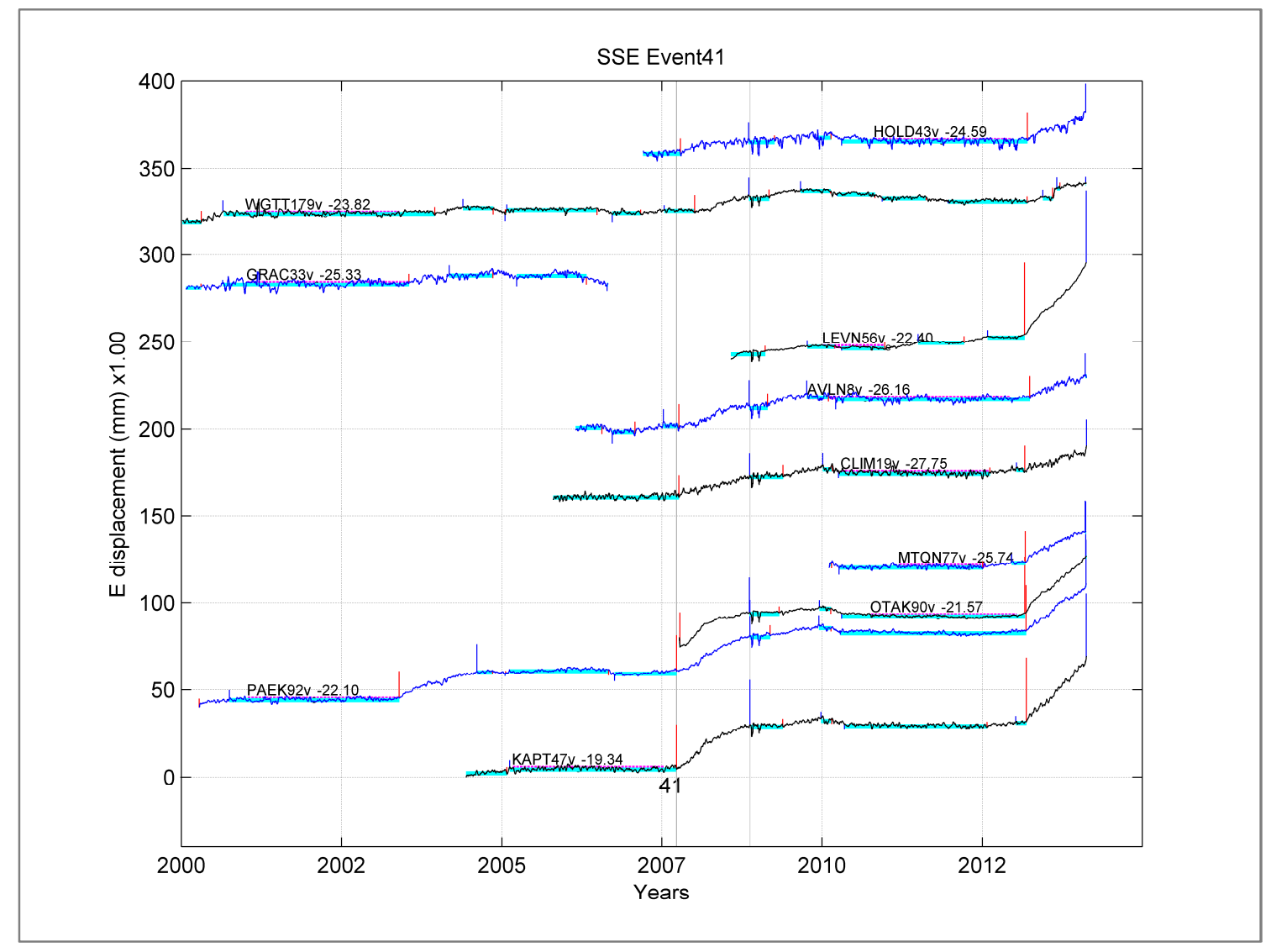

Figure 1.1: Example of a plot targeting an SSE occurring over the period 2007-08, evidenced in detrended east component measurements at seven cGPS sites. Each trace is offset by $50 \mathrm{~mm}$ for clarity and is ordered by distance from the site with the largest displacement, which is plotted closest to the abscissa, and alternate between black and blue for ready trace identification. Vertical grey lines indicate the earliest start and latest end-times for this event, which is identified by the event number (41) shown on the $1^{\text {st }}$ trace. Each trace is identified by the site's GeoNet code, accompanied by the secular velocity of the segment initially used to de-trend the trace. Short red and blue lines respectively indicate picked start and finish times of events. Alternating azure and cyan horizontal lines mark sections deemed to be SSEfree - see Section 2.4.

\subsection{Documented occurrences of SSEs}

SSEs have been increasingly documented around the Pacific Rim subduction zones. By 2007, Schwartz and Rokosky [2007] were able to catalogue published data on events in Costa Rica, Mexico, the West Coast of USA and Canada (Cascadia), Alaska, Japan, and New Zealand. Since then, slow slip events have also been documented in Taiwan $[C$ Liu et al., 2009], Ecuador [Nocquet et al., 2009; Vallée et al., 2013], and Peru [Villegas Lanza et al., 2012]. Additionally, slow slip associated with earthquake preslip and 
afterslip has been documented in some of these locations, plus Kamchatka [Bürgmann et al., 2001], and suspected to be present in the central Philippines Fault system [Besana and Ando, 2005]. Despite the suggestion of slow slip preceding the 1960 M8.3 Chile earthquake, Kanamori and Cipar [1974] attempts to discover aseismic slip in Chile using data from Interferometric Synthetic Aperture Radar (InSAR) appeared to have been unsuccessful [Holtkamp et al., 2011]; but more recently an SSE was detected just prior to the April $2014 \mathrm{M}_{\mathrm{w}}$ 8.1 Iquique event [Ruiz et al., 2014].

As indicated in section 1.1, SSEs have been observed to be associated with nonvolcanic tremor, and initially were called 'Episodic Tremor and Slip' (ETS). Tremor is detected in seismograms and has been known to be associated with volcanic activity. It has the general characteristics, compared with tectonic earthquakes, of long duration (lasting minutes to hours), lack of impulsive arrivals, lower dominant frequencies, and an often harmonic character. The "new" tremor was not associated with volcanoes and was usually recurrent (episodic), and generally not harmonic. Whilst tremor and slow slip are closely associated in Cascadia [Wech, 2010] and in parts of Japan [Obara, 2011], in other areas tremor occurs without detectable slip [E F Smith and Gomberg, 2009], or slip occurs without detectable tremor [Delahaye et al., 2009], and hence the term ETS is not now preferred. In areas where there is adequate data resolution, SSEs can be detected at varying depths and distributions along subduction interfaces, and may be shown to be migratory, and heterogeneous in areal extent (patchy) [Bartlow et al., 2014].

\subsection{Principal characteristics of SSE zones}

Cartoons of subduction interfaces (e.g. Obara [2011] ) tend to show (Fig. 1.3):

- A "locked" zone of "brittle" interface - the conventional earthquake zone - down to approximately $20 \mathrm{~km}$

- Very low frequency earthquakes (VLF) in the depth range 0 to $5 \mathrm{~km}$ (in the accretionary prism)

- Short-term SSEs (lasting days) in the depth range 10 to $20 \mathrm{~km}$ (Boso Peninsula, Gisborne-Hawkes Bay, Costa Rica, Ecuador)

- Short-term SSEs associated with tremor in the depth range 30 to $45 \mathrm{~km}$ (Cascadia, Nankai) 
- Long-term SSEs (lasting months) in the depth range 35 to $60 \mathrm{~km}$ or more (Cascadia, Kapiti-Manawatu, Alaska)

- A zone of intra-slab earthquakes extending from $25 \mathrm{~km}$ downwards.

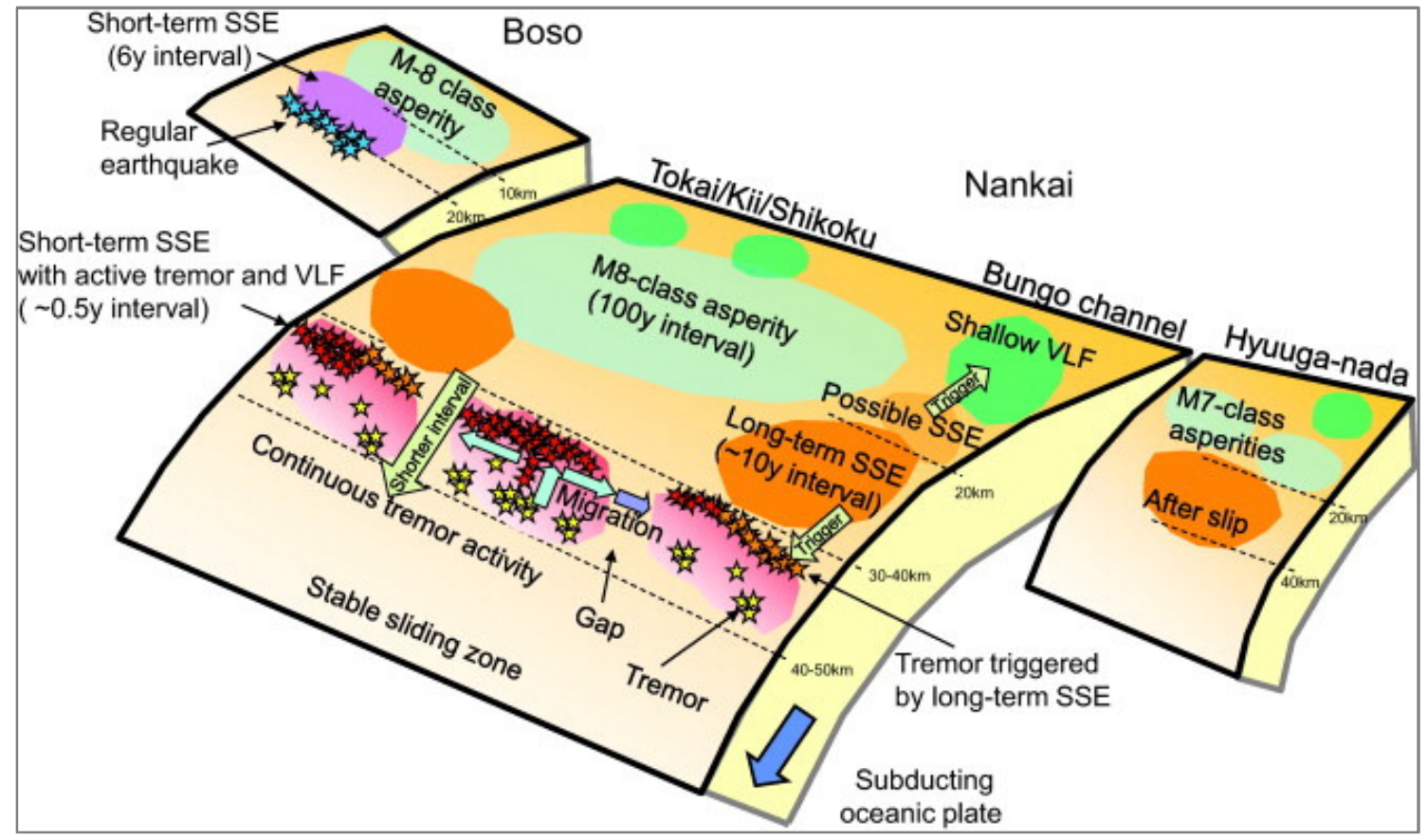

Figure 1.3 (from Obara, 2011): Schematic illustration of slow earthquake activity along the subducting Philippine Sea Plate. The plate boundary is divided into three regions, representing different combinations of slow earthquakes. In the Boso region, short-term SSEs recur at intervals of 6 years, at the downdip side of the asperity associated with regular earthquake swarms. Along the Nankai trough, shallow VLF earthquakes occur in spatial clusters. From the Tokai to Bungo Channel regions, at the deepest part of the transition zone, short-term SSEs recur at intervals of 6 months, associated with active tremor and deep VLF earthquakes. The coupled phenomena are divided into segments. Within each segment, active tremor bursts are mainly concentrated at the updip edge of the tremor zone; downdip tremor, on the other hand, is characterized by frequent recurrence. Tremor migration usually starts at the downdip edge of the tremor zone and propagates laterally. On the updip side of the tremor zone, longterm SSEs are located on both edges of the tremor zone; the long-term SSEs trigger tremor activity in adjacent locations. In the Bungo Channel region, long-term SSEs might extend to the shallower side of the subduction zone and trigger shallow VLF earthquakes. In the Hyuga-nada region, after-slip events were detected at the downdip side of the asperity, following an M 6.7 interplate earthquake. (Note that this conceptual depiction predates the M9 Tohoku-oki event and understanding continues to evolve, e.g. Yokota and Koketsu [2015].)

However, this superficial view hides much detail that currently precludes generalisation, and is still being updated (see, for example, a plot of widely-varying depth ranges, Fig. 8 in Fu and Freymueller [2013]. It has also been suggested that both SSEs and megathrust earthquakes can occur on the same fault area [Noda and Lapusta, 2013; Yokota and Koketsu, 2015]. 
In many cases, depths of SSEs are poorly constrained by the existing instrumental coverage, and assumptions are made based on interface models that have primarily been inferred from seismic data. There is, for example, some indication that aseismic slip can occur in the shallow (accretion) zone at the depths over which most thrust earthquakes on subduction interfaces occur [E Davis et al., 2006; Hyndman, 2007].

\subsection{Tectonic setting of the Hikurangi margin}

The Hikurangi subduction margin comprises a segment of the Pacific-Australia plate interface situated between the ocean-ocean subduction along the Tonga-Kermadec trench to the north, and the continent-continent transpression along the Alpine Fault in the South Island [Seebeck et al., 2013], see Fig 1.4.

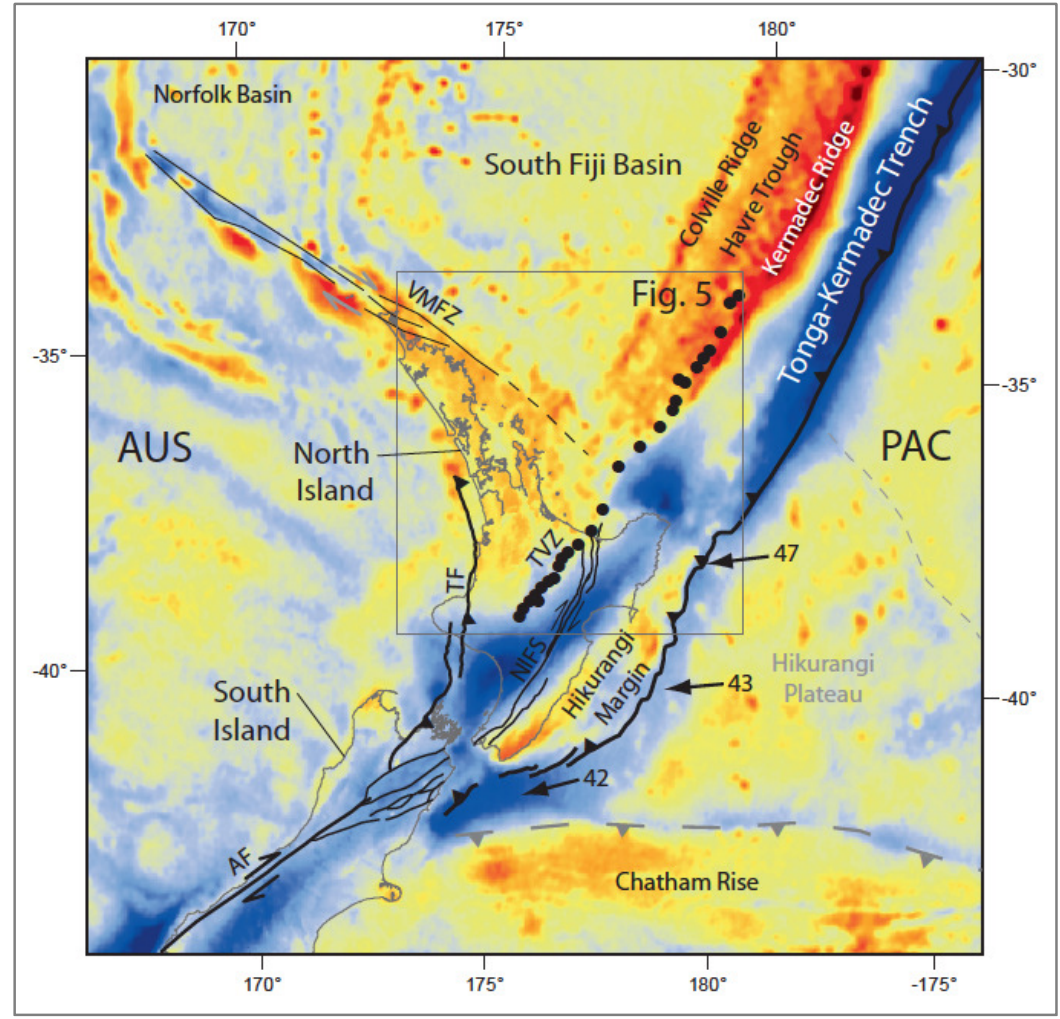

Figure 1.4 (from Seebeck et al., 2013): Present tectonics and arc-front volcanism of the New Zealand plate boundary superimposed on satellite free air gravity [Sandwell and Smith, 2009]. The Hikurangi subduction margin forms a transition from oblique westward ocean-ocean subduction along the TongaKermadec trench to continental collision and transform motion along the Alpine Fault. Miocene to Recent crustal-scale faults (black lines): AF, Alpine Fault; TF, Taranaki Fault; VMFZ, Vening Meinesz Fracture Zone; NIFS, North Island Fault System. Present arc-front volcanic centres (black filled circles): TVZ, Taupo Volcanic Zone. Black arrows indicate Pacific plate (PAC) motion relative to a fixed Australian plate (AUS; in mm a-1; Beavan et al. 2002). 
Along the east coast of the North Island, thickened oceanic crust of the Hikurangi Plateau is subducting approximately westwards at a rate varying from approximately 47 $\mathrm{mm} /$ year in the East Cape region (margin normal), to approximately $42 \mathrm{~mm} / \mathrm{year}$ in the Marlborough region (margin parallel) [Nicol and Wallace, 2007]. Extension in the Taupo Volcanic Zone and the Havre Trough further north, results in clockwise rotation of the North Island forearc.

Subduction occurs along the Hikurangi Margin interface, which is relatively young (post $30 \mathrm{Ma}$, [Furlong and Kamp, 2009]) compared with most subduction zones. The interface was first characterised by seismic data, described in [Ansell and Bannister, 1996], subsequently updated in C A Williams et al. [2013]. Both models use a degree of smoothing that may overlook important detail [Salmon et al., 2011] that might help explain apparent anomalies in deformation fields. An alternative but similar geometry has been proposed and described by Lamb and Smith [2013]. The two geometries are compared in Appendix 6.

\subsection{SSEs along the Hikurangi subduction zone}

Short ( 10 days), shallow ( 10 to $15 \mathrm{~km})$ SSEs with a recurrence period of 2 to 3 years were first documented near the Raukumara Peninsula by Douglas et al. [2005]. This discovery was shortly followed by that of a large (equivalent to $\mathrm{M}_{\mathrm{W}} \sim 7.0$ ), long ( 18 mths), and deep ( 35 to $60 \mathrm{~km}$ ) SSE in the Manawatu region [Wallace and Beavan, 2006].

Subsequently, Beavan et al. [2007] described further shallow $(<15 \mathrm{~km})$ events detected beneath Hawkes Bay, and another deep (30 to $40 \mathrm{~km}$ ) event beneath the Kapiti Coast. The latter was hypothesized to have triggered a swarm of earthquakes 25 to $30 \mathrm{~km}$ below Upper Hutt [Reyners and Bannister, 2007].

Delahaye et al. [2009] looked for tremor linked with the Raukumara peninsula events but found only an increased level of reverse fault micro-seismicity. Their analyses 
included sensitivity assessment of the network and concluded that this should have been capable of detecting tremor of similar amplitude to that found in Cascadia.

Wallace and Beavan [2010] updated the list of SSEs detected in the Hikurangi subduction zone. They summarised that the Gisborne-Hawkes Bay events were similarly shallow $(10-15 \mathrm{~km})$, short $(<2$ weeks), with recurrence in the range 1 to 2 years, and of equivalent magnitudes in the range $\mathrm{M}_{\mathrm{W}} 6.3$ to 6.8. They suggested that these events took place in a region of weak, shallow inter-seismic coupling. In the Marlborough-Kapiti-Manawatu region they summarised that the events were deeper (25 to $60 \mathrm{~km}$ ), of longer duration (> 200 days), with longer recurrence (4.5 to 6.5 years), and larger in terms of moment release $\left(\mathrm{M}_{\mathrm{W}} 6.6\right.$ to 7.2). They suggested that all events were taking place downdip of strongly locked portions of the interface, consistent with the along-strike variation of interseismic locking proposed by Beavan and Wallace [2008] and Wallace et al. [2009].

Fry et al. [2011] searched for events triggered by the Feb 2010 Maule Chile $\mathrm{M}_{\mathrm{W}} 8.8$ event, and detected tremor associated with the region and depth of the Manawatu SSE. Kim et al. [2011] reported detection of tremor associated with the 2010 Gisborne SSE, and hypothesized that tremor always accompanies SSE, but may not always be detectable where there are highly-attenuating sediments present. Chao [2012] reported detecting triggered tremor beneath a point approximately midway between Lake Taupo and Hawkes Bay following the $2011 \mathrm{M}_{\mathrm{w}} 9.0$ Tohoku-Oki earthquake. Ide [2012] reported detecting almost 100 small tremor events at 20 to $50 \mathrm{~km}$ beneath the central North Island.

Wallace et al. [2012a] reported apparent triggering of a series of small shallow earthquakes in 2010-11 by a slow, deep, mainly eastwardly-migrating SSE beneath Manawatu. The earthquakes commenced updip beneath Castlepoint (south of Hawkes Bay), and appeared to migrate northwards up the coast to offshore Gisborne. Significant migrating microseismicity was also associated with periods of this SSE migration. They suggested that prior earthquake cluster activity in 2006, and perhaps 2001, might also have been associated with SSEs in the southern Hawkes Bay region, although there was sparse or no GPS coverage in the earlier periods. They suggest that the small static Coulomb stress changes likely to have been associated with the larger 
SSE could be of similar magnitude $(1-10 \mathrm{kPa})$ to dynamic stress changes suggested to be associated with triggering of tremor by distant earthquakes.

Tremor lasting 12 days was detected by Wech et al. [2012] 25 to $45 \mathrm{~km}$ beneath the Alpine Fault zone, and was inferred to imply an SSE on a plate boundary transitional fault, similar to tremor events described for the San Andreas Fault, where geodetic signals were also absent.

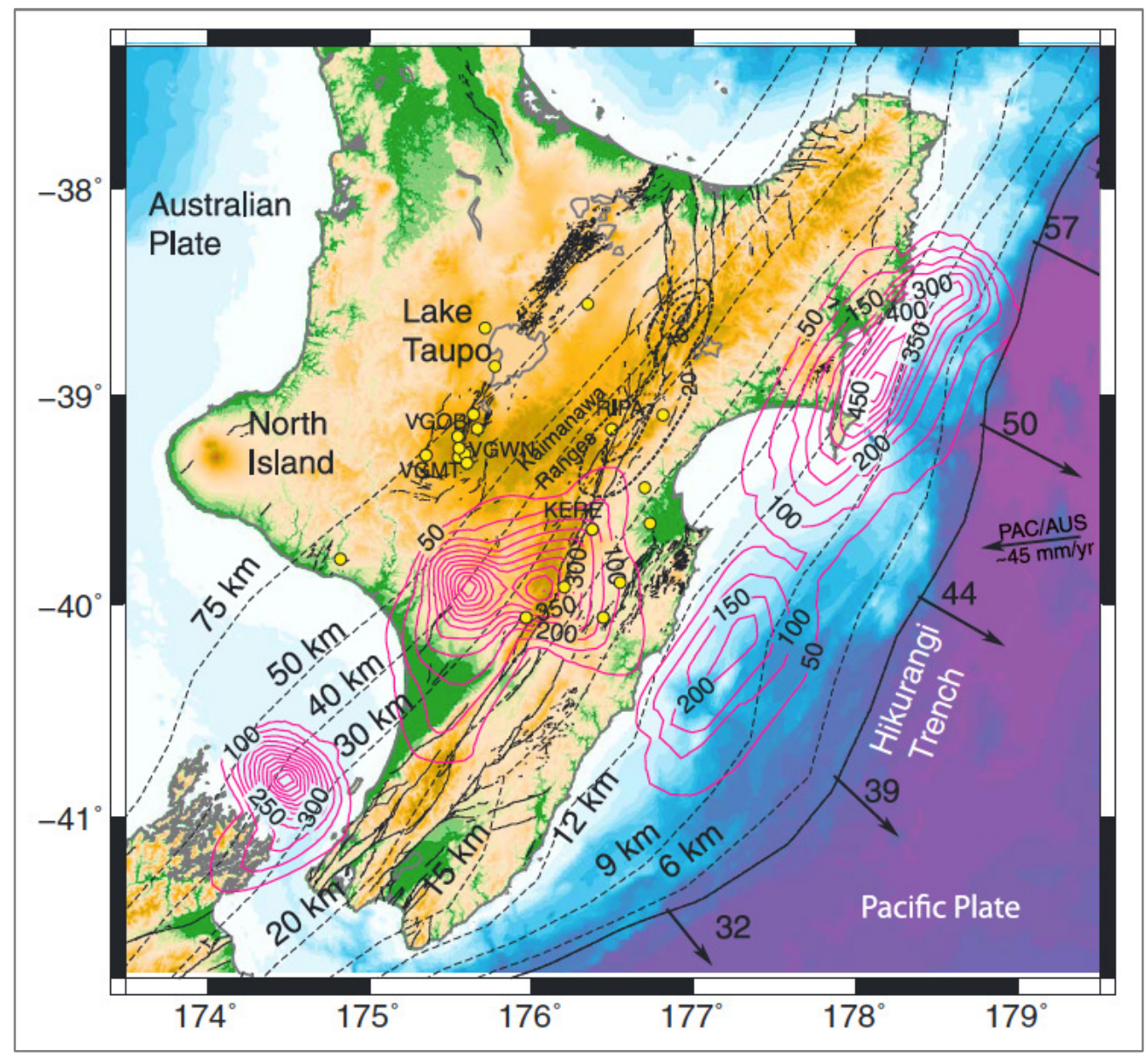

Figure 1.5 (from Wallace and Eberhart-Phillips, 2013): Locations of larger SEEs on Hikurangi margin. Convergence rates at the Hikurangi trench are shown in mm/yr. [Wallace et al., 2012b]; dashed black lines show the subduction interface geometry and are labeled with depth (in kilometers) [C A Williams et al., 2013]. The cumulative slip in slow slip between 2002 and 2012 (with the exception of the deep SSEs discussed in this paper) from Wallace et al. [2012a] shown as magenta contours (contours labeled in $\mathrm{mm}$ ). Dotted black contours near the Kaimanawa Ranges show the position of the 2008 deep SSE that Wallace \& Eberhart-Phillips introduce in their paper; note its position well downdip of previously recognized shallow SSEs at the central Hikurangi margin. Yellow dots show the cGPS sites used to identify the 2006 and 2008 deep SSEs. The single arrow, centre right, indicates the overall secular velocity of the Pacific Plate with reference to the Australia Plate. Note that in 2006-2008, the GeoNet cGPS network was in the process of being developed and had fewer sites compared to the large number of sites that are now operating. 
Deep events occurring in 2006 and 2008, downdip of the shallow East Coast sequence, were described by Wallace and Eberhart-Phillips [2013], together with a more recent large shallow event offshore of southern Hawkes Bay. Fig. 1.5, taken from their paper, illustrates the locations and context of the more significant events.

The dynamic nature of East Coast SSEs was illustrated in Bartlow et al. [2014]. The authors modeled the time-dependent behavior of 12 SSEs occurring during 2010 and 2011, and in the supporting material to the publication, showed animations of the apparent temporal evolution and movement of the slip patches.

In summary, the North Island SSEs described to date in the literature include:

- Deep, slow events in at least 4 zones along a northeast trend from offshore Marlborough through to the Kaimanawa Ranges and Raukumara peninsula.

- Shallow, more rapid events along a north-east trend from offshore Castlepoint to Gisborne

The locations of the events recorded to date do not now strictly follow the outline of the 'locked zone', as had been intimated in some of the earlier publications.

\subsection{Interpretations of SSEs in New Zealand}

Clearly the interpretation of the New Zealand events has evolved over time as more data became available. This brief review therefore concentrates on the later publications.

As indicated above, the published data to date show both deep long-duration events and shallow short events, with a gap between (see, for example, Fig. $4 \mathrm{~b}$ in Wallace and Eberhart-Phillips [2013], reproduced as Fig. 1.6). 


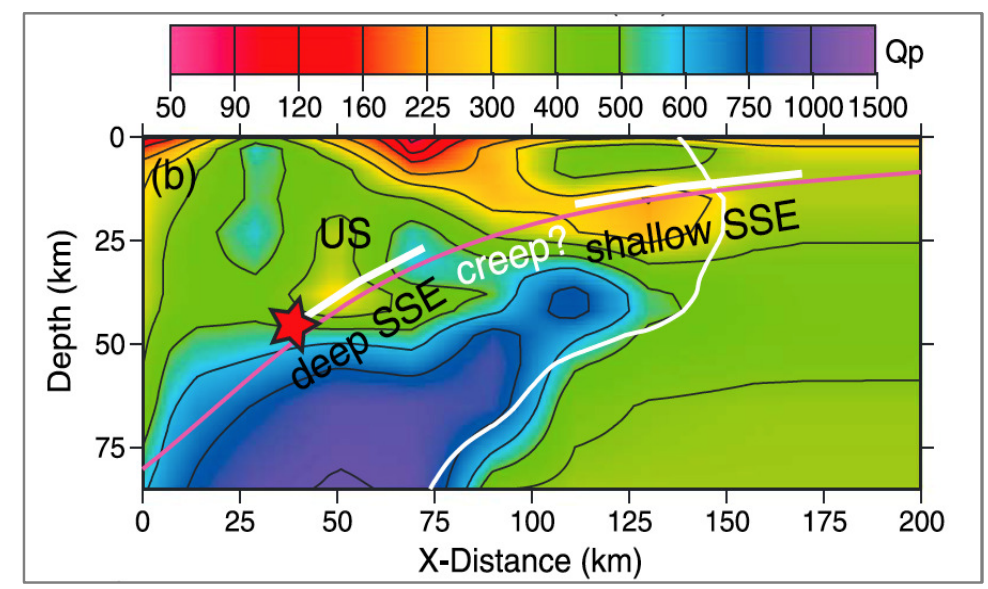

Figure 1.6 (reproduced from Wallace \& Eberhart Phillips, 2013): Cross section of inverse attenuation, Qp, across the Hikurangi SSE source region (from Eberhart-Phillips et al., 2008). Both deep and shallow SSE slip areas on the interface are shown with heavy, white line, and the subduction interface (from Williams et al., 2013) with a magenta line. The cross-section runs perpendicular to the trench, along a line from approximately 50km SE of Lake Taupo, through Cape Kidnappers (at $\sim 90 \mathrm{~km}$ along X) to the trench at $X=200 \mathrm{~km}$.

With the assumption that the events take place on or close to the subduction interface, as modeled in C A Williams et al. [2013], Wallace and Eberhart-Phillips imply that in the north-east, between approximately 15 and $25 \mathrm{~km}$ depth, there is a band that creeps aseismically. It is unclear whether this is continuous creep or a series of SSEs that so far have been too small to be detected. Depths are generally not well constrained, especially where the events are indicated to have taken place below offshore regions. For the shallow events, the $2013 \mathrm{M}_{\mathrm{W}} 7.0$ equivalent magnitude SSE is indicated to have filled what was earlier noted as a gap between the shallow events along the east coast.

For the deeper events, associated ambient and triggered tremor has been documented. At this stage, the tremor has not been associated in an episodic manner similar to ETS described in other Pacific regions. Tremor has been located downdip of SSEs, perhaps suggestive, according to Wallace and Eberhart-Phillips [2013], of pressure-temperature constrained dehydration reactions or specific rheological conditions. The lack of temporal association with geodetically-determined SSEs is suggested as implying the possibility of deeper small SSEs that cannot be resolved by the GPS network, similar to Cascadia, where there appears to be a continuum of slip size and periodicity - however, in contrast, in Cascadia there is a continuum of event depth, which, to date, does not 
seem to be the case in New Zealand. It is concluded that the subduction interface is highly heterogeneous in frictional properties.

In the source areas of the SSEs, several studies indicate conditions that would support the hypothesis of the presence of high-pressure fluids - high Poisson's ratios (high $\mathrm{V}_{\mathrm{p}} / \mathrm{V}_{\mathrm{s}}$ ) [Bannister et al., 2007; Reyners et al., 2006]; high attenuations (low $\mathrm{Q}_{\mathrm{p}}$ ) [Eberhart-Phillips et al., 2008]; and low to intermediate resistivity [Heise et al., 2012]. It has also been proposed that correlation between high rates of subducting slab seismicity and the spatial extent of rhyolitic volcanic systems suggest that elevated fluxes of slab-derived fluids into the mantle wedge is a key driver for the high volcanic production rates observed in the TVZ [Seebeck, 2012]. However, a cautionary note is suggested by modeling studies [Mitsui and Hirahara, 2008], in which it is commented that whilst high Poisson's ratio may be produced by high fluid saturation (as might be expected from metamorphic dehydration), this doesn't necessarily mean higher pore pressures (which depend also on low permeability). (Higher pore pressures have been suggested as a mechanism for lowering effective stress and promoting slip [Kodaira et al., 2004].) There appears to be no clear consensus on what happens to fluid carried down into subduction zones, although accumulation of serpentinite in the forearc mantle, and silica accumulation in the lower crust are common themes [Ramachandran and Hyndman, 2012].

The subducting plate at Hikurangi is old [E G C Smith et al., 1989; $R$ Wood and Davy, 1994] and therefore relatively cold. Thermal conditions have been investigated and dismissed as probable explanatory variables of variations in coupling along the margin [Fagereng and Ellis, 2009; McCaffrey et al., 2008].

Active seismic data have been used to characterise the subduction thrust geometry along the east coast of the North Island. On the basis of reflection seismic data indicating the presence of several subducted seamounts, Bell et al. [2010] suggested a conceptual model for the Gisborne region:

"low effective mean stress (could be produced) due to enhanced under-thrusting of fluid-rich sediment, reduced overburden stresses where the interface has been warped to shallower depths to accommodate seamount subduction; and 
potential fluid flow concentration effects leading to overpressure along these shallower interface corrugations." (p. 46)

Fagereng [2011] examined subducted wedge geometry down the east coast, and concluded:

"based on critical Coulomb wedge theory, the shallow megathrust appears relatively weak in the locked segment, and stronger in the weakly coupled regions. (abstract)

Thus no casual attempts should be made at correlating 'strongly' coupled faults, capable of large to great earthquakes, with faults experiencing high shear stress. I therefore suggest that in some subduction zones, high fluid pressure and low temperature allow for frictional failure to depths at least down to the Moho (e.g. Cascadia; Wang et al. (1995), and Southern Hikurangi; this study), but if fluid pressure is low, high effective stress causes other failure mechanisms, such as distributed cataclasis coupled with dissolution-precipitation creep, to be dominant over frictional faulting (e.g. northern Hikurangi). In this case, frictionally weak subduction megathrusts are strongly coupled, and possibly fail in episodic large earthquakes, while frictionally strong faults tend to deform by distributed microseismicity or aseismic sliding." (p. 29)

Similar observations to those of Fagereng have also been made by X Gao and Wang [2014] and K Wang and Bilek [2014]. Gao and Wang suggested that:

"extreme ruggedness of the subducting sea floor such as at the northern Hikurangi gives rise to heterogeneous stress and structural environments that promote creep and small earthquakes". (p. 1040)

Conversely, they suggest that, from heat-flow measurements and modeling, that smooth interfaces, accompanied by deeper sediments and weak gouge, may give rise to characteristic stick-slip behavior that results in large earthquakes.

Whilst not referring specifically to the Hikurangi, Kaneko et al. [2010]; Wei et al. [2013] suggested that spatial frictional heterogeneity may play a large part in explaining variation in seismic and aseismic behaviour on large faults, and that regions of low interseismic coupling may reveal barriers to large earthquakes.

No clear explanation for the totality of observed events has therefore yet emerged. 


\subsection{Thrust earthquakes on the Hikurangi subduction interface}

It has been widely observed that there have been no megathrust earthquakes along the Hikurangi within historical time. Instrumental records only became available from the 1930's, and reliable eyewitness accounts from about the 1840's.

The largest regional earthquake within historical time was the $M_{w} 8.21855$ Wairarapa event, which ruptured $120 \mathrm{~km}$ of the Wairarapa fault but was primarily strike-slip motion within the overlying plate [Rodgers and Little, 2006] - although there has been some suggestion that it could have reached down to the subduction interface [Beavan and Darby, 2005]. There have been no interface thrust events on the Hikurangi, documented in the last 170 years, that have exceeded $\mathrm{M}_{\mathrm{w}} 7$ [Doser and Webb, 2003; Heuret et al., 2011; Webb and Anderson, 1998], and whilst there have been a number of intraplate events greater than $\mathrm{M}_{\mathrm{w}} 6$, few of these involved pure thrust [Downes et al., 1997; Hamling et al., 2014; Robinson, 1994].

Fig. 1.7 indicates the locations of subduction thrust events recorded in the North Island within historical time, taken from Wallace et al. [2014].

Drawing parallels with the March 2011 Tohoku-Oki earthquake and the nature of coupling along the Japan subduction interface, Wallace et al. [2014] suggest that a megathrust event of up to $\mathrm{M}_{\mathrm{w}} 9$ would not be unimaginable for the Hikurangi margin. Whilst not indicating a potential magnitude, Webb and Anderson [1998] expressed a similar view that, whilst the margin has a relatively low level of seismicity, it is accumulating elastic strain that will eventually be released in large earthquakes. Scholz and Campos [2012] model seismic coupling for a generic subduction margin using a parameter called the 'seismic flux accumulation rate', and in their discussion of specific margins argue that for the northern Hikurangi, where seismic coupling is low, megathrust earthquakes are unlikely, and whilst not stated, one may therefore surmise that for the southern part of the margin, where coupling is said to be high, this implies that such large earthquakes are feasible. McCaffrey [2008] argued that, in time, all subduction zones should be capable of generating $\mathrm{M}_{\mathrm{w}} 9$ earthquakes, but other authors, e.g. Heuret et al. [2011], seem not as convinced. 


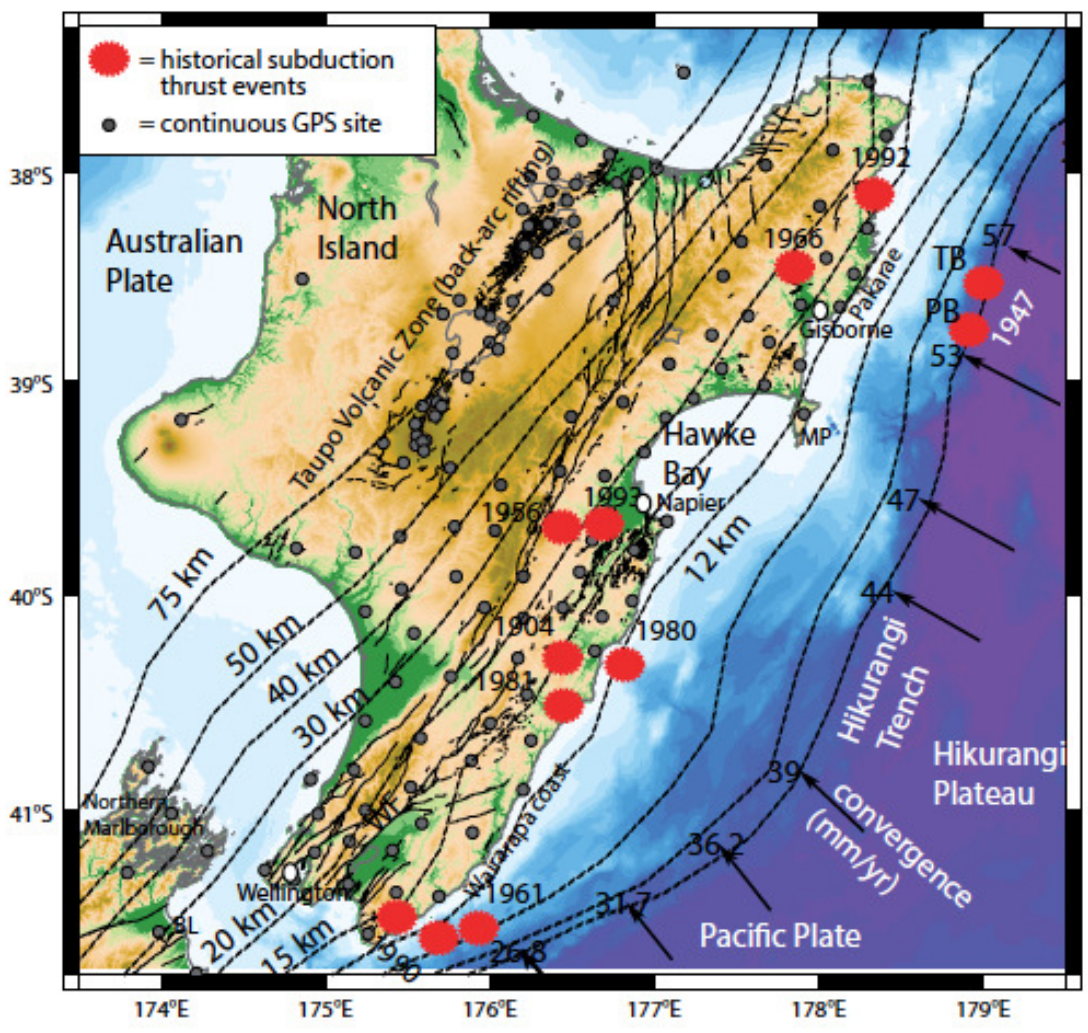

Figure 1.7 (as Fig. 1 in Wallace et al. [2014]): Tectonic setting of the Hikurangi subduction zone at the boundary between the Pacific and Australian Plates. Black contours show the depth to the subduction interface [C A Williams et al., 2013]. Red dots = historical subduction thrust events (all $5.6<\mathrm{Mw}<7.2$ ). Gray dots = continuous GPS sites (http://www.GeoNet.org.nz). Arrows show convergence rates at the trench in $\mathrm{mm} \mathrm{yr}^{-1}$ [Wallace et al., 2012a]. PB = 1947 Poverty Bay earthquake. $\mathrm{TB}=1947$ Tolaga Bay earthquake. $\mathrm{WF}=$ Wairarapa Fault, the site of the 1855 earthquake. BL $=$ Big Lagoon. MP $=$ Mahia Peninsula. Black lines onshore are active faults (http://www.data.gns.cri.nz/af). In the forearc, most of these faults are either right lateral strike-slip or reverse. The strike-slip faults help to accommodate the margin-parallel component of relative plate motion.

Prior to 1840 , there is some evidence in Maori oral tradition of a large earthquake in 1460 causing uplift in the Wellington area [D N T King et al., 2010], but geological evidence for this having been a megathrust event is sparse [Clark et al., 2011]. To date, the best estimate of when the last megathrust event took place, based on widespread terrace uplifts and lagoon subsidence along the east coast from Turakirea Head (SE of Wellington) to Pakarae (NE of Gisborne), was about 7100 years ago [Wallace et al., 2014]. 


\subsection{Objectives of this research}

The Hikurangi subduction margin presents some unique features that are not well explained. Observations of SSEs span a wide range of depths, durations, periodicity and magnitudes, and with this in mind, the generation of a catalogue of events as complete as possible can only assist in future investigations, including understanding temporal stress changes along the Hikurangi margin.

The objectives of the research are to:

1) Develop methods for the routine processing of GeoNet continuous GPS data for determining the location, moment, mechanism and duration of Slow Slip Earthquakes (SSEs) along the Hikurangi margin, and the uncertainties of these quantities. These methods build on work done by a number of authors in New Zealand and worldwide to quantify SSEs, but will focus on routine application to the New Zealand cGPS dataset.

2) Process the existing cGPS catalogue to produce a catalogue of SSEs that is as complete and homogenous as possible.

3) Use the SSE catalogue to address two questions:

(a) What are the fundamental differences in SSE properties in the north-eastern and south-western Hikurangi margin?

(b) In particular, is the difference in temporal evolution of NE SSEs (shortduration) and SW SSEs (long-duration) controlled by a simple timescale variable?

The catalogue as produced contains limitations that are discussed later. It has not been possible within the time constraints of the project to carry out a full analysis of the catalogue, nor was that one of the primary objectives. Whilst some preliminary analyses have been carried out, further work is ongoing to address the questions posed above in greater detail. 



\section{Methods}

\subsection{Continuous GPS - introduction}

The Global Positioning System (GPS) arose from the NAVSTAR System, and comprises a satellite-based radio-positioning and time-transfer system, designed, financed, deployed, and operated by the U.S. Department of Defense [Misra and Enge, 2006].

In the face of continued growth and diversification of GPS applications, the worldwide scientific community promoted the development of international standards for GPS data acquisition and analysis, and to deploy and operate a common, comprehensive global tracking system.

As part of this effort, the International GPS Service (IGS) was formally recognized in 1993 by the International Association of Geodesy (IAG), and began routine operations on January 1, 1994, providing GPS orbits, tracking data, and other data products in support of geodetic and geophysical research. In particular, since January 1994, the IGS has made available to its user community the IGS official orbit based on contributions from the seven current IGS Analysis Centers.

The IGS collects, archives, and distributes GPS observation data sets of sufficient accuracy to meet the objectives of a wide range of scientific and engineering applications and studies. These data sets are used to generate the following products [IGS, 2013]:

- GPS satellite ephemerides

- GLONASS satellite ephemerides

- Earth rotation parameters

- IGS tracking station coordinates and velocities

- GPS satellite and IGS tracking station clock information

- Zenith tropospheric path delay estimates

- Global ionospheric maps

Continuous Global Positioning System (CGPS) networks for geophysical applications began in the early 1990's on a global scale with the IGS, to provide a global terrestrial 
reference frame, and on a regional scale to monitor plate boundary deformation and the hazards that they pose. Campaign-mode GPS measurements during the 1980's proved valuable for studies of seismic cycle deformations; however, the limited temporal resolution of the measurements, generally obtained at any one site over acquisition periods of the order of hours, motivated the transition to continuous operation [Crowell, 2013].

Continuous measurement allows signal-to-noise levels to be much improved by averaging - raw data, acquired at rates up to 1 measurement per second and with relative precision of metre scale, are reduced to daily solutions with a relative precision on the centimeter scale, which, when analysed over periods of days, reduces the precision to millimetre scale, assuming that the location remains stationary. Fig. 2.1 shows typical cGPS station setups at Nelson and Cape Palliser in New Zealand.

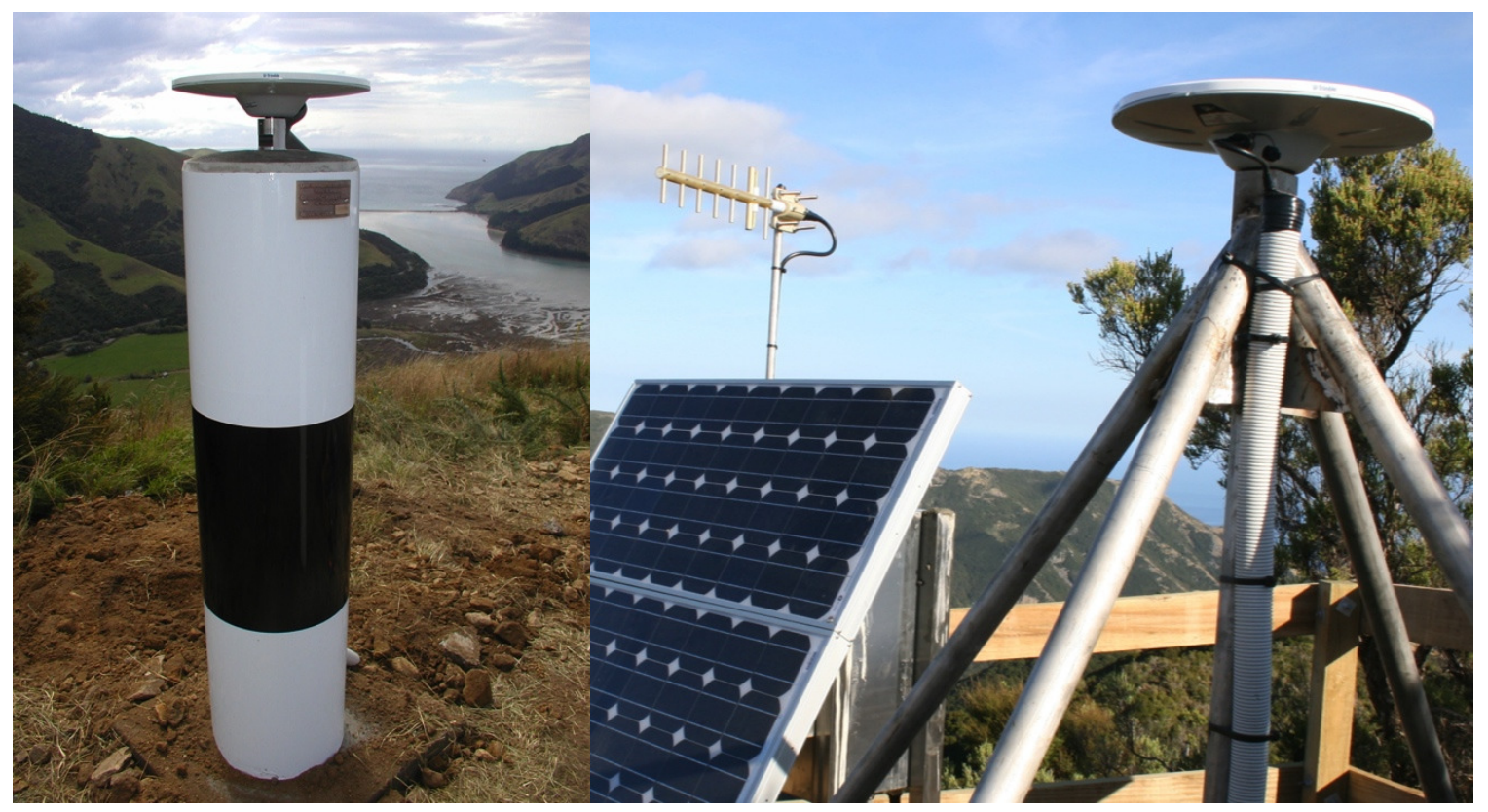

Figure 2.1 (courtesy GeoNet): Two typical cGPS station setups - at Nelson, showing the concrete monument and antenna; and at Cape Palliser, showing the antenna mounted on a grouted braced monument, the solar power supply, with signal processing and data transmission unit in the rear. 


\subsection{GeoNet cGPS data}

In New Zealand, cGPS data are provided by GeoNet, a service comprising a network of geophysical instruments, operated by Geological and Nuclear Sciences (GNS), and funded by the Earthquake Commission (EQC) and Land Information New Zealand (LINZ). Fig. 2.2 shows most of the extent of the onshore GeoNet cGPS network as at Feb 2014.

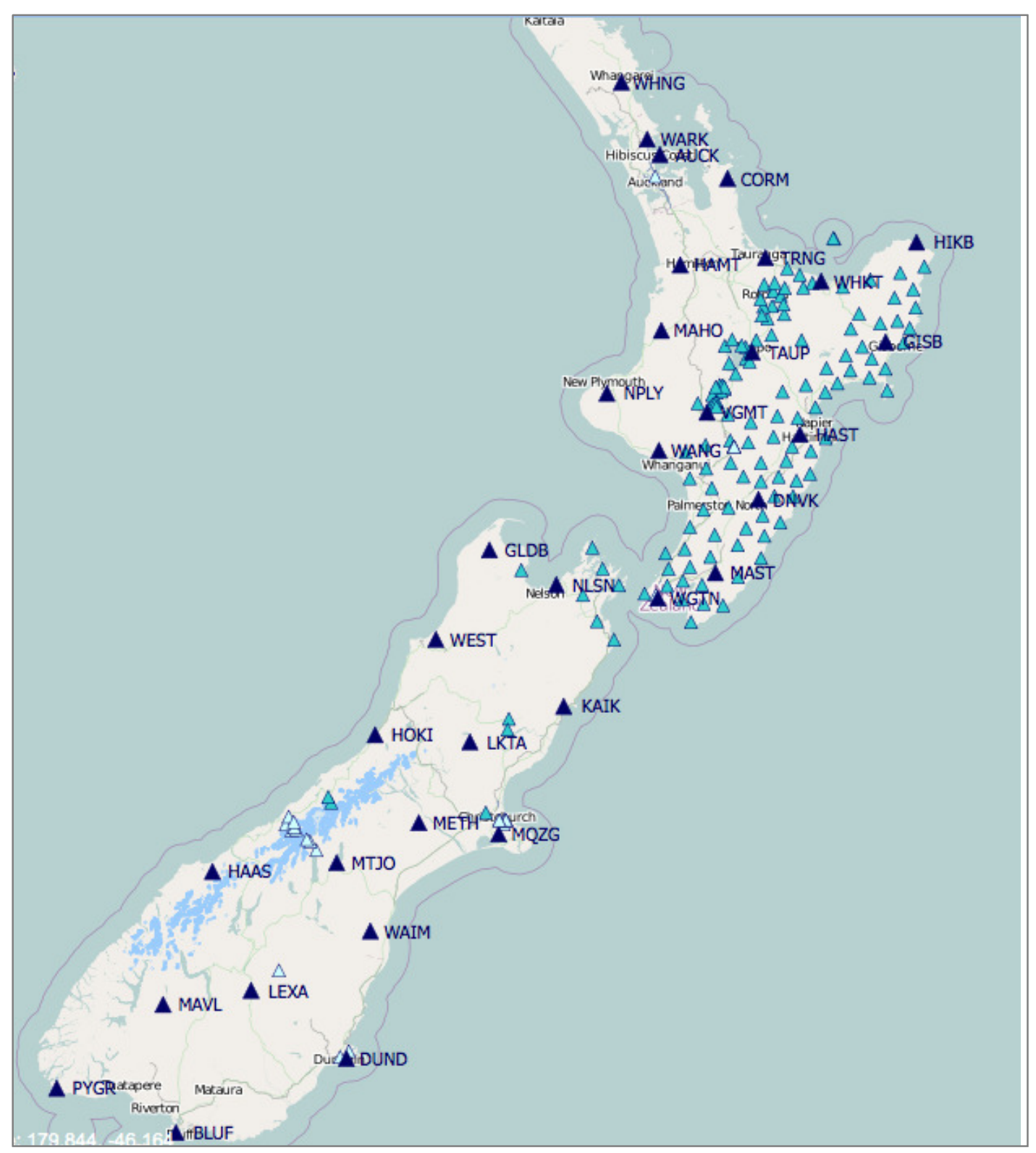

Figure 2.2 (courtesy GeoNet): Approximate extent of the cGPS network in New Zealand at Feb 2014. Named sites (dark blue triangles) are part of the LINZ permanent geodetic network. Cyan triangles indicate the locations of cGPS sites, and pale blue triangles indicate temporary installations. 
According to GeoNet [Geonet, 2013], the preliminary GPS processing is carried out:

"using Bernese v5.0 software developed by the Astronomical Institute of the University of Bern (AIUB), using relatively modern models. See Wallace and Beavan (2010) and references within for a more detailed processing explanation. In summary, the processing strategies are as follows:

- Solutions calculated each day for data 1, 3, 30 and 90 days behind real time;

- IGS final orbits and earth orientation parameters for data older than 2 weeks;

- IGS rapid orbits and earth orientation parameters for data more recent than 2 weeks;

- Orbits are not solved for:

IGS relative antenna phase patterns from PHAS_COD.IO8 (downloaded from AIUB);

Satellite antenna phase patterns;

- Ocean loading from ocean tide model TPXO.7.1 calculated by Onsala Space Observatory;

- Global ionospheric model downloaded daily from AIUB;

- Solution done as $\sim 11$ clusters, each of $<\sim 20$ stations, that are combined using ADDNEQ2;

- Solution aligned to ITRF2008 realisation, using a 3-parameter Helmert transformation onto a subset of IGS sites in central and eastern Australia, the southwest Pacific and the Antarctic. (For an explanation of the relationship between IGS and the ITRF2008 global reference frame, see [Bosy, 2013])

Three files that are essential for the New Zealand GPS processing are the a priori coordinate and velocity files, and the site information file.

The coordinates and their formal uncertainties are extracted from daily solution files, and converted to (east, north, up) displacement in millimetres from an initial point.

- For stations in the LINZ PositioNZ network, the initial point is LINZ's NZGD2000 position of the GPS station.

- For other New Zealand stations, the initial point is either the NZGD2000 position of the station, or is the first point in the time series (a long-term aim is to use the NZGD2000 position for all stations).

Some "outlier" data points, which are far different to the neighbouring data points, are removed from the time series using an automated procedure. The resulting "raw" time series have no other adjustments made to them, so they may, for example, contain offsets due to earthquakes, offsets due to equipment changes at individual sites, and seasonal signals due to various causes. They also contain a "common-mode" noise signal that is reasonably constant across a wide region. This may arise from the use of slightly erroneous satellite orbits, regional-scale or global-scale mass redistributions (in the ocean, atmosphere or groundwater), the use of non-optimal models in the daily processing, and perhaps other causes." 
This raw daily time series data has been utilized for all further processing undertaken as part of this project.

\section{3 cGPS sites utilized in this project}

At the time of commencing this work, there were 224 cGPS site names listed by GeoNet. Of these, 29 had been closed for some time, 6 were located overseas, and 14 were temporary installations, leaving 175 permanent installations. In order to focus on the Hikurangi margin, sites were selected in a swath from NE to SW of the N. Island, and including a number of sites in the NE of the S. Island. Most sites in the Taupo Volcanic Zone (TVZ) were excluded, since these are known to be affected by regional tectonics (Taupo back-arc and Hikurangi fore-arc spreading) [Darby and Meertens, 1995], although sites around Ruapehu were included. In all, 115 sites were included in the processing.

An additional 17 sites were included in a western group, from both islands, which were judged stable and used for reference to reduce regional effects.

The 115 selected sites are shown in Fig. 2.3a against a background of the surface geology [NZGS-GNS, 2011]. Appendix 1 shows the geological legend for this figure.

It should be remembered throughout this work that the number of cGPS sites has increased from 2000, and therefore coverage in the early part of the period was much less dense than towards the end of the $1^{\text {st }}$ decade. This has a bearing on both the increased ability to detect SSEs over time, and on the precision of average secular velocities measured at each site. Fig. $2.3 \mathrm{~b}$ summarises the growth in the number of sites available during the period covered by the project. 


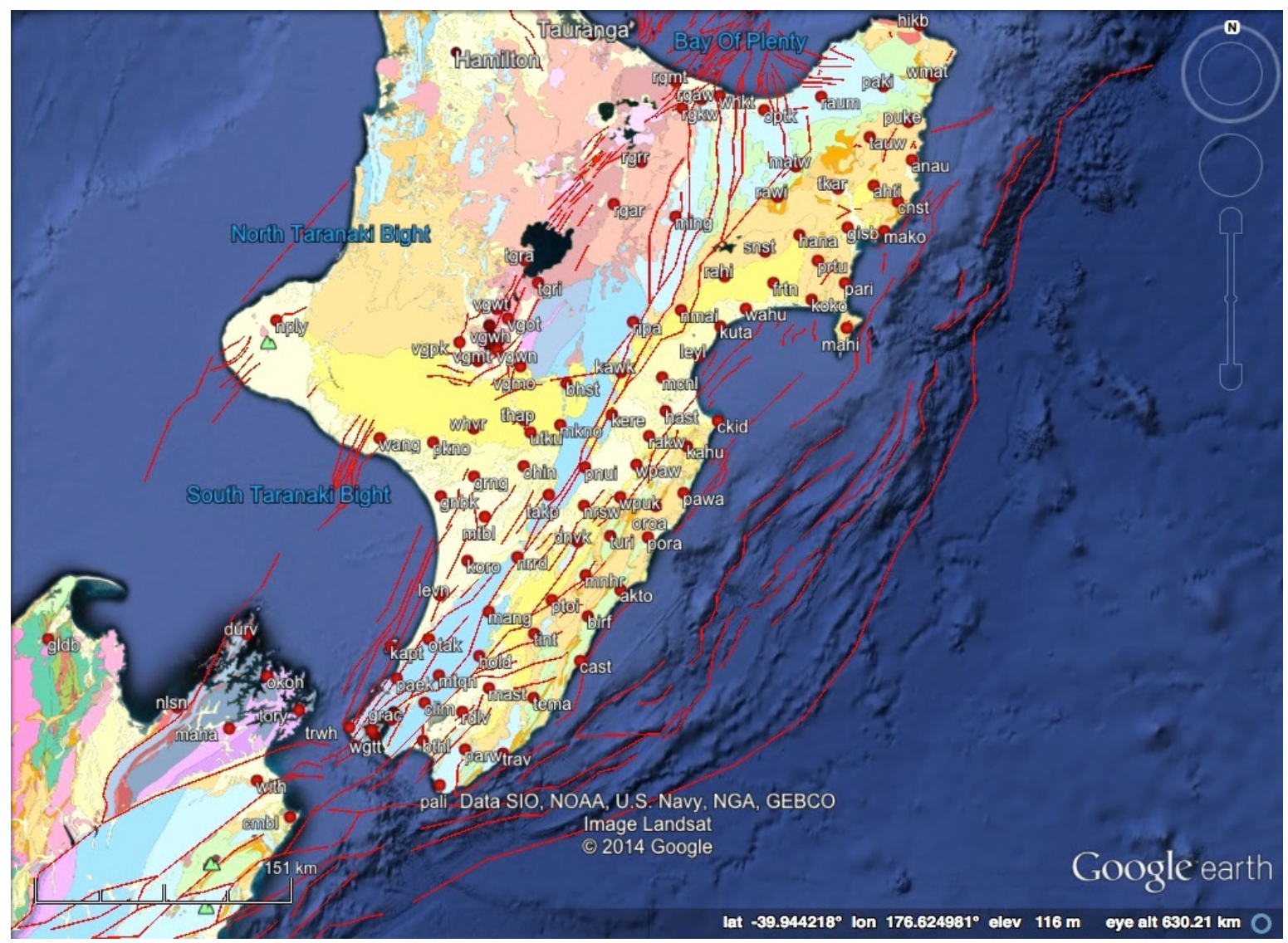

Figure 2.3a: cGPS sites used in this study, shown against the surface geology (see Appendix 1 for lithology) and active faulting [Litchfield et al., 2014]. Background image courtesy Google Earth, Surface geology courtesy GNS Science.

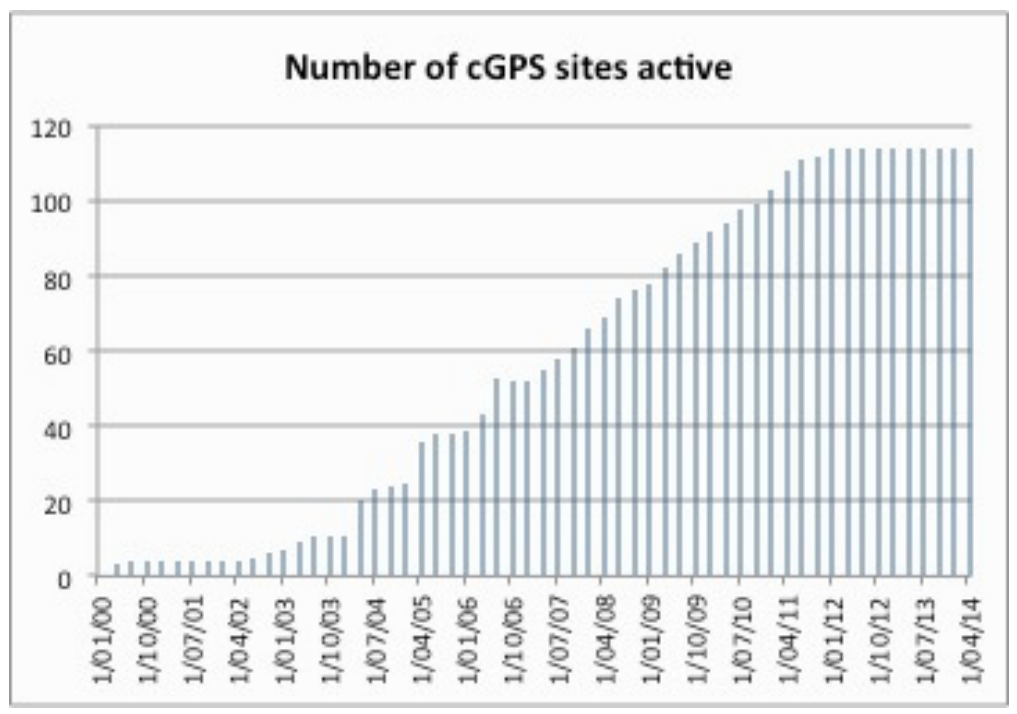

Figure 2.3b: Growth by quarter in the number of cGPS sites available in the area shown in Fig. 2.3a during the period covered by this project. 


\subsection{Processing methodology}

\subsubsection{Data management}

Daily solution files for the 3 components for all cGPS sites were downloaded from the GeoNet site on 16 Feb 2014. These comprise the raw data for this project. Each file, containing the mean daily displacement solution and standard deviation for each day, was loaded to a Matlab array, indexed by site and component, with a filter to generate an additional file which indexed all missing data (or, to be more precise, an index of all 'good' data). GPS clock times were converted to Gregorian calendar days and then indexed from 01 Jan 2000, which was taken as the first day on which cGPS monitoring commenced in New Zealand (although, in fact, the first site was brought on line on 03 Jan 2000).

\subsubsection{Noise reduction}

Fig. 2.4.2 shows an example of the raw data for the east component from site AUCK.

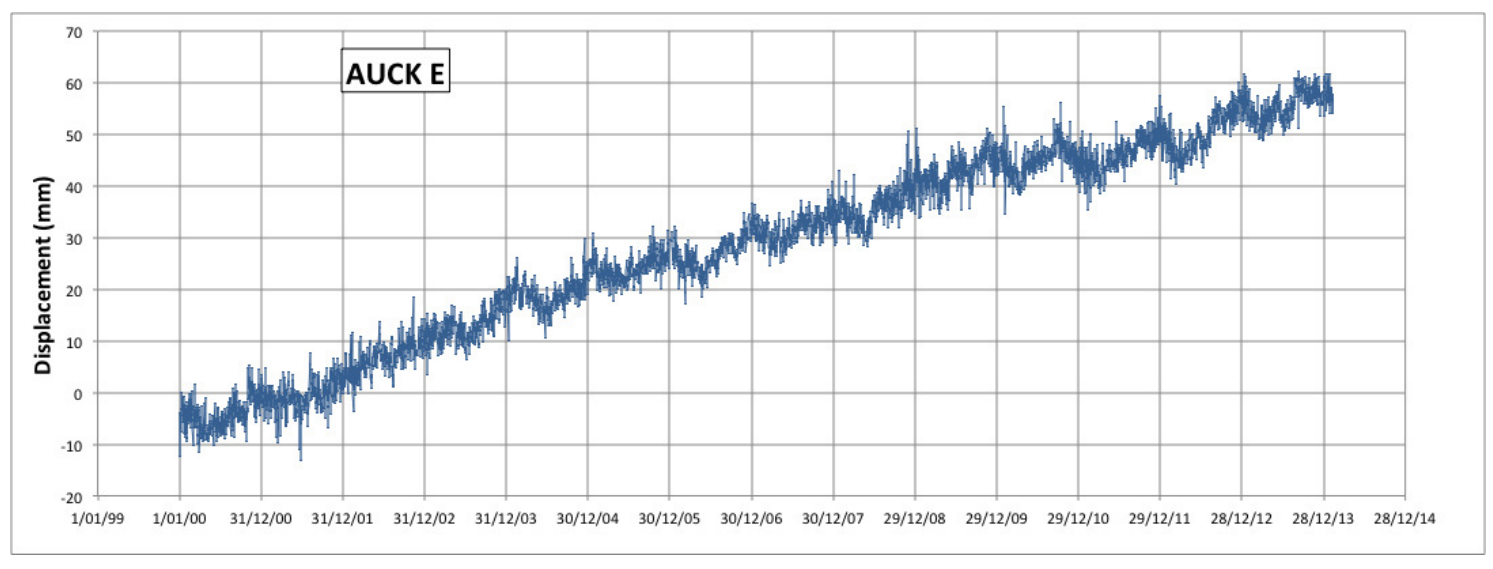

Figure 2.4.2: An example of raw daily cGPS data - east component, site AUCK

It can readily be seen that the raw daily solution data show an approximately linear trend, together with seasonal deviations. Additionally, the data are relatively noisy.

GPS data may be affected by many potential sources of noise, including mis-modeling of satellite orbits, un-modeled atmospheric effects, correlation through estimated parameters within the GPS data processing, monument instability and environmental 
factors, multipath effects, mis-modeled antenna phase centre effects and instrumentation noise [M A King and Williams, 2009; Lidberg et al., 2007; Mao et al., 1999; Santamaría-Gómez et al., 2011]. Whilst some noise is purely random (white noise), other components are time-correlated (coloured noise) [Dermanis and Kotsakis, 2005; S D P Williams, 2003], such as seasonal periodicity (which may have a variety of potential causes [J L Davis et al., 2012]). Meertens et al. [2012] also highlight some possible contributory factors:

- mountain sites - changes due to surface water loading - subsurface moisture changes - these should be seasonal, and should affect $\mathrm{U}$ rather more than $\mathrm{N}$ or $\mathrm{E}$ components - however, this is also true of many other sites subject to changes in hydrological conditions. Snow loading may be much more variable.

- antenna deterioration - this is likely to show as diurnal noise due to poorer temperature compensation, but where signals are averaged over 24 hours, effects will be variable.

- monument stability noise - this was still being studied by Meertens et al. [2012], but deep drilled \& braced monuments were said to be the most stable. Beavan [2005] indicated that NZ concrete geodetic monuments were also stable and probably as good. There is no evidence in our data of any systematic difference. Both deep braced and concrete pillars are used in the NZ network.

In order to resolve geophysical phenomena, it is therefore important to eliminate or reduce as many of these noise sources as possible without reducing the SSE signals [Beavan, 2005; Calais, 2010].

A generalized schema of the noise processing code is shown in Appendix 2. This has been implemented in Matlab by Prof. E. Smith. An example of the output from this processing was shown above in Fig. 1.1.

Several aspects of this processing deserve further examination:

\subsubsection{Noise components}

As indicated above, there are a number of potential sources of noise in cGPS data, as also indicated in the description of the GeoNet processing in 2.2 above. Noncorrectable tropospheric delay is a major factor. Misra and Enge [2006] indicated that tropospheric delay cannot be compensated by dual frequency instruments and relies on 
modeling of atmospheric conditions. This is, of course, complicated in windy mountainous areas, where weather is known to vary within a few kilometres, and correction is probably not very practical in many locations in New Zealand. It is therefore unlikely to be removed by regional network bias.

\subsubsection{Regional noise}

Some models for regional noise assume fixed annual and semi-annual cycles - this assumption requires that whatever causes these signals must be fixed in amplitude and phase, which therefore cannot allow fully for (stochastic) environmental effects that are caused primarily by weather cycles [J L Davis et al., 2012]. Alternative methods for reducing seasonal noise in GPS data have been documented by Bennett [2008] and Borsa et al. [2014]

In the Matlab code, the removal of common-mode noise or 'regional filtering' is accomplished by stacking the de-trended data from 17 stations situated along the western side of the network (which are relatively free of influence from SSEs), for the period 2000 to 2014, excluding periods where the effects of large earthquakes are noted (Fiordland M7.2 Aug 2003, George Sound M6.7 Oct 2007, Dusky Sound M7.8 Jul 2009, and Darfield M7.1 Sep 2010).

Outliers in each of the data series incorporated in the stack are down-weighted using a Jeffreys' weighting algorithm (see below). Fig. 2.4.2.2 shows the three component time series in the stack. This stack is then removed (subtracted) from all time series in subsequent processing.

The composition of noise in the stack is of interest. This was examined using Fourier time series analysis [StatSoft, 2013]. Appendix 3 shows the spectral content for each component. It can be observed that the greatest amplitude in the E component is at close to an annual period, with much lower amplitudes at semi-annual and 120 day periods. The $\mathrm{N}$ component shows a much weaker annual and semi-annual peak. The U component illustrates a wide range of peaks, at bi-annual, annual, the strongest at semiannual, 3 months, and possibly 2 -month periods. The character of the noise, as implied by the spectral index (gradient of the log-log plot), would in each case be described as mostly 'pink' or 'flicker' noise, with only a small amount of white noise evident in the E component for periods below approximately 10 days. 

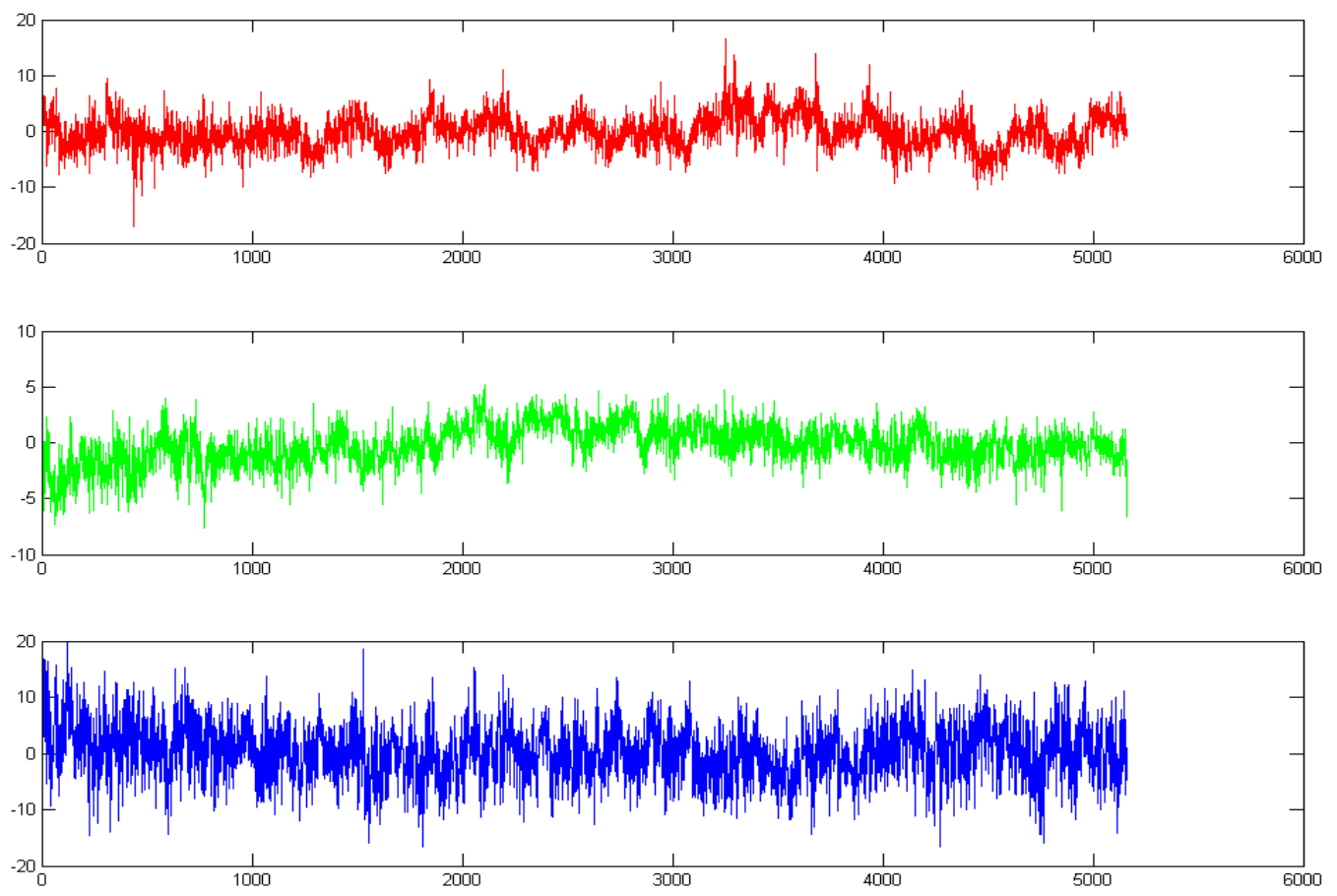

Figure 2.4.2.2: Regional noise levels in the 17-site stack, with the 3 components, top to bottom being east $(\mathrm{E})$, north $(\mathrm{N})$ and vertical $(\mathrm{U})$. The ordinates are in $\mathrm{mm}$, and the abscissa is in days from $01 \mathrm{Jan}$ 2000 .

\subsubsection{Residual seasonal noise}

Once each time series has been regionally filtered, there remains some residual 'seasonal' signal that also needs to be removed. This is illustrated in Fig. 2.4.2.3, which shows the 30-day moving average residual 'seasonal' signal for the two sites in Auckland.

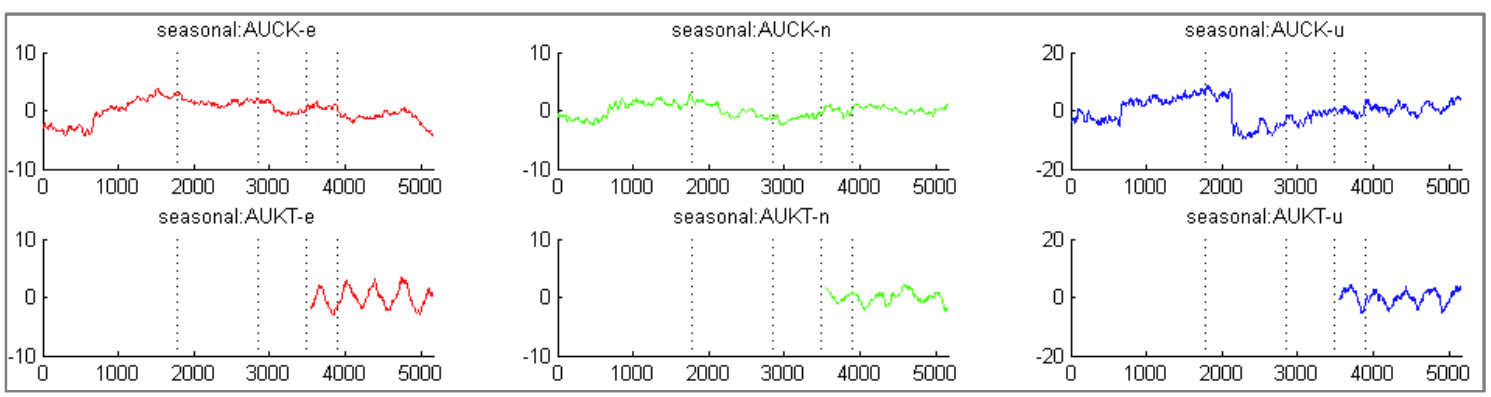

Figure 2.4.2.3: Residual seasonal time-series for the 3 components at the 2 Auckland sites after removal of the regional effects, but before removal of seasonal effects. 
It can be observed that despite elimination of a significant annual component by the regional filtering, there still remained an annually-periodic residual at AUKT, which is removed in this step. In the Matlab code, this is achieved by calculating the 9-day moving average for each de-trended time series, and deducting this from each series. Earlier work had showed that this step did not affect the ability to detect SSEs [Barrett, 2014].

\subsubsection{Outlier removal}

Outlier removal is especially important in GPS processing. Whilst major outliers have been removed in the daily solution processing by GNS, other outliers remain, and an objective method is required for assessment and removal. Again, care is required to ensure that SSE signals are not compromised.

Whilst most authors accept that outliers exist in GPS time series, there is no general consensus as to how they should be treated. In many papers, whilst acknowledging that outliers have been removed, the methods of detection, and decision processes, are often not detailed by the authors.

Outliers can arise from GPS data quality problems, multipath and other site-specific errors, atmospheric refraction, and the incorrect choice of integer-cycle phase ambiguities. Large spikes can arise when satellites just rise above the elevation cut-off, since atmospheric refraction models at low elevation are relatively poor. Incorrect choice of integer-cycle phase ambiguities result in stepwise systematic offsets in horizontal coordinate time series with little effect on the vertical time series [Bock et al., 2000], and so should be relatively easy to pick, although both of these effects apply more to sub-daily than daily time-series. Complex interplay of multipath and tropospheric refraction results in more random scatter in both horizontal and vertical series, but with greater amplification in the vertical.

A number of authors refer to a simple model of the form:

$$
f\left(t_{i}\right)=a+b t_{i}+c \sin \left(2 \pi t_{i}\right)+d \cos \left(2 \pi t_{i}\right)+e \sin \left(4 \pi t_{i}\right)+f \cos \left(4 \pi t_{i}\right)+\sum_{j=1}^{n_{g}} g_{j} H\left(t_{i}-T_{g j}\right)+\varepsilon_{i}
$$

where: $\boldsymbol{f}(\boldsymbol{t})$ is the observed displacement in each of the 3 directions, $\boldsymbol{t}_{\boldsymbol{i}}$ are the daily solution epochs (in yearly format), $\boldsymbol{a}$ is an initial offset, $\boldsymbol{b}$ is the linear rate 
(in tectonic studies, this secular velocity component is often regarded as the "answer"), $\boldsymbol{c}$ and $\boldsymbol{d}$ are coefficients with respect to an annual periodicity, and $\boldsymbol{e}$ and $\boldsymbol{f}$ similarly for a semi-annual periodicity; and the penultimate component is the Heaviside function for any number $\left(\boldsymbol{n}_{\boldsymbol{g}}\right)$ of offsets with amplitude $\boldsymbol{g}$ and epochs $\boldsymbol{T}_{g}$, and the final term $\varepsilon_{i}$ is the assumed white noise component.

It will be noted that this model implies fixed seasonal effects, which may not be completely appropriate - in long-term series, it will be observed that there appears to be phase drift on some of these seasonal signals, which is to be expected if they are due to a combination of draconitic ${ }^{\underline{1}}$ harmonics and environmental effects.

In processing data from disparate European networks in order to examine long-term trends in Italy, Serpelloni et al. [2006] refer to the "Robust" outlier procedures included in the QOCA software [Bock et al., 2000]. However, the latter authors recommend that GPS data be processed instantaneously (i.e. on each 30 second interval) rather than on a daily batch-wise basis, claiming that outliers are easier to deal with at that stage. (This also confers the advantage that noise data can be examined above the limiting daily Nyquist frequency of $0.006 \mathrm{mHz}$, and thus allowed the authors to be able to attribute flicker noise to tropospheric effects.) Their "Robust" method, again referring to baseline comparisons, is simply based on defining outliers as anything within a single epoch lying outside the range median $+/-3 x$ interquartile range, which appears rather crude, but they indicate that over a 12 week period this eliminates only $1.8 \%$ of the data on a $50 \mathrm{~m}$ baseline, $4.6 \%$ for a $14 \mathrm{~km}$ baseline, and $16.8 \%$ for a $37 \mathrm{~km}$ baseline. They then used temporal stacking to minimise common mode effects. They consider that using instantaneous processing would allow campaign GPS to occupy sites for much shorter periods for equivalent precision. (They also mention that the 11-year sunspot activity cycle can also affect GPS data.)

Tolvi [1999] summarised the issues with outliers in time series, principally looking at economic data. However, amongst a wide range of options, he recognised the existence

\footnotetext{
$\underline{1}$ Wikipedia definition: The draconitic period, or draconic period, is the time that elapses between two passages of the object (in this case GPS satellite) through its ascending node, the point of its orbit where it crosses the ecliptic from the southern to the northern hemisphere. This period differs from the sidereal period because both the orbital plane of the object and the plane of the ecliptic precess with respect to the fixed stars, so their intersection, the line of nodes, also precesses with respect to the fixed stars. Although the plane of the ecliptic is often held fixed at the position it occupied at a specific epoch, the orbital plane of the object still precesses causing the draconitic period to differ from the sidereal period.
} 
of mixture models, "where the deviant observations can be thought of as outliers originating from a contaminating distribution", which is a similar idea to that of Jeffreys [1932]. He mentioned that early ideas revolved around examining higher moments such as kurtosis. He noted that there are many methods of dealing with outliers and it is hard to make an objective choice between them, since, to a large extent, they all depend on a subjective assessment of what constitutes an outlier. However, he did stress that early identification of outliers is important in any modelling situation.

Gökalp et al. [2008] evaluated conventional and robust methods as well as fuzzy logic for detecting outliers in GPS data, but principally in geodetic baseline measurements. They concluded that all the robust methods (except Yang-II M-estimation) produce more outlying observations than conventional statistical test methods.

Wright [2008] set out to maximise resolution in seismic studies of deep structure in South Africa. In determining station corrections, he examined use of weighted data computed using an adaptation of the method of uniform reduction [Bullen, 1963; Jeffreys, 1932; 1961] in order to minimise the effects of outliers. In a lengthy discussion, the author proposed using kurtosis of the weighted distribution as a test of the degree of weighting required.

Jeffreys [1932] initially proposed the following error mixture model (which was subsequently simplified to the "method of uniform reduction", in order to simplify the calculations, which at that time had to be undertaken manually):

The distribution of the probability of error is therefore taken to be

$$
f\left(x_{r}\right)=(1-m) \frac{h}{\sqrt{ } \pi} e^{-h^{2}\left(x_{r}-x\right)^{2}}+m \frac{k}{\sqrt{ } \pi} e^{-k^{3}\left(x_{r}-x-y\right)^{\mathrm{a}}} .
$$

This expresses the conditions (1) that if $x$ is the true value the probability, given $x$, that an observation will lie between $x_{r}$ and $x_{r}+d x_{r}$ is $f\left(x_{r}\right) d x_{r} ;(2)$ the probability that an observation will be affected by the abnormal error is $m$; (3) the normal observations follow a normal law with modulus of precision $h$; (4) the abnormal ones follow another normal law with modulus $k$, but are subject also to an unknown systematic error $y$. Our problem is to find the most probable value of $x$, and incidentally $y$, from a given set of observed values $x_{1}, x_{2}, \ldots, x_{n}$.

The advantage of using Jeffreys' weighting is that data are not deleted, but simply down-weighted. An immediate task is therefore to find an optimum value of $\boldsymbol{h}$ in the 
Jeffreys' model. In the Matlab code, $\boldsymbol{h}$ is expressed via a multiplier of the root mean square (rms) of the residuals of each time series $\left(\mathbf{h}^{*}=\mathbf{h} \sqrt{ } 2\right)$. In practice, the distribution of sorted residuals is split into two halves, so that each can be separately treated, thereby also reducing any significant skewness.

Kurtosis gives an indirect measure of the outlier content of a distribution, in that it parameterises the deviation from normality of the distribution, both in the 'peakedness' of the distribution and 'heaviness' of the tails.

The optimum value of the Jeffreys' weighting parameter, $\boldsymbol{h}$, has been calculated by determining the kurtosis of the residuals following application of the weighting. A normal distribution has a kurtosis value of 3.0, and therefore this is the target outcome. The value of the multiplier $\boldsymbol{h}^{*}$ was varied from 0.85 through to 2.0 and at each level, the entire data set was run through the weighting process and the kurtosis calculated for each site. For sites with the full complement of data (5160 days), it was calculated that the standard error of the kurtosis distribution should be $+/-0.068$, and therefore any value of kurtosis outside the range 2.86 to 3.14 for these sites could be considered to be significantly different to 3.0 at the $95 \%$ confidence level.

Kurtosis should be normally distributed, but there still remained some 'outliers'. Figure 2.4.2.4a shows the kurtosis of Jeffrey-weighted residuals for the sites plotted against the kurtosis of the residuals prior to weighting, for three different values of the weighting parameter $\boldsymbol{h}^{*}$. A value of $\boldsymbol{h}^{*}=2.0$ was effectively the same as applying unity weighting for most of the sites. It was consequently decided to use the median of the kurtosis values as a measure of location, rather than the mean, for the purpose of tracking the consequence of changing the value of $\boldsymbol{h}$.

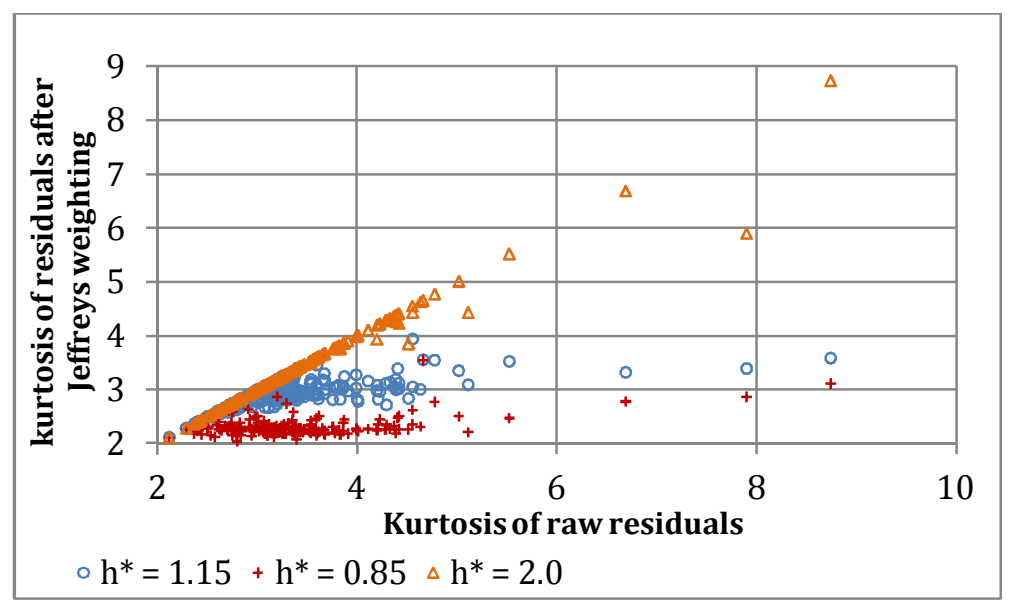


Figure 2.4.2.4a: Comparison of kurtosis of E-component residuals on sites before and after application of the Jeffreys' weighting, for 3 different values of the weighting coefficient $\mathbf{h}^{*}$

It was observed that the effect was different on the $\mathrm{E}$ component to the $\mathrm{N}$ and $\mathrm{U}$ components, as can be seen in Figure 2.4.2.4b.

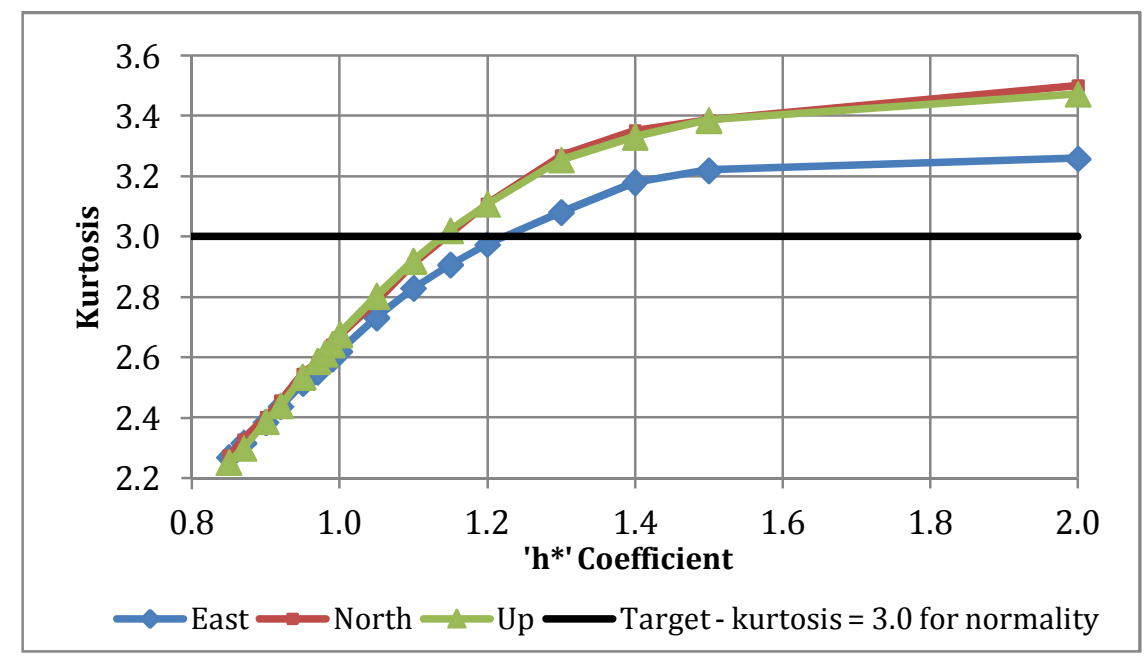

Figure 2.4.2.4b: Median value of kurtosis as a function of the coefficient $\mathbf{h}^{*}$ for all sites for 3 components. The target value of 3.0, which represents the kurtosis value for a normal distribution, is shown as a heavy black line.

From Fig. 2.4.2b, it was decided that the value of $\boldsymbol{h} *$ to be applied would be 1.22 for the E component and 1.14 for the $\mathrm{N}$ and $\mathrm{U}$ components (equivalent to Jeffreys' $\mathbf{h}$ values of 0.863 and 0.806 respectively).

Appendix 4 illustrates the Jeffreys' weighting application on an example site, and the overall effect on rms residuals.

\subsubsection{Smoothing}

Smoothing is carried out by the simple application of a 9-point moving average, weighted to avoid missing data. This span had been determined previously [Barrett, 2014] as suitable to avoid disguising the onset of any significant slow slip event.

\subsubsection{SSE identification and picking}

The identification of SSEs and other discrete events has been carried out manually using Matlab 'Display' codes written by Prof. E Smith and described in detail by Barrett 
[2014]. At the start of this project, approximately 55 events had already been picked by Rachel Barrett.

In summary, the de-trended, noise-reduced east-component traces are displayed in groups. The east component traces are used for picking since they have the highest signal-to-noise ratios. For each site, a section of trace that displays no sign of an SSE is chosen in order to initially set the non-slip secular velocity (see Fig. 1.1). The groups of sites are indexed by detected events, and the number of sites displayed is then determined by a criterion based on the distance of the surrounding sites from the one showing the largest amplitude for that event. Clearly this is an iterative process in which events showing at different sites need to be matched across sites.

Matching was carried out based on when the event was first identified in the time series. The identification was aided by the use of cursors and trace magnification, with the aim being to tag the times at which the event appears to commence and finish, or stabilize, on a particular trace. This then generated an index of events at each site, which were assessed for correspondence with events at other sites. Figure 1.1 shows a typical set of traces. The start and completion of each event on each trace is marked with red and blue spikes respectively, so that when traces from nearby sites are shown together, it can be ascertained as to whether these are likely to be have been generated by the same source. The radius of assessment can be altered, so that initially, relatively large numbers of traces can be scanned to ensure that displacements from a single source event have been captured out to the furthest distance, and to ensure that the same source event does not get identified twice in two adjacent geographic areas. To assist in this process, sites associated with each SSE were mapped so that anomalies could be more easily identified. Fig. 2.4.3 shows an example of such a set of maps for a group of events.

It should be noted that the early attempts to identify SSEs at multiple sites assumed logarithmic time dependence (loosely based on a conceptual parallel with the temporal distribution of aftershocks from earthquakes). It soon became clear that an exponential fit to the data was just as feasible, and hence these two functions were used in all the early code. Only later did it transpire that some events showed clear evidence of slow starts rather than abrupt take-off, and hence further work was later carried out to examine the temporal development of events (section 2.5). 


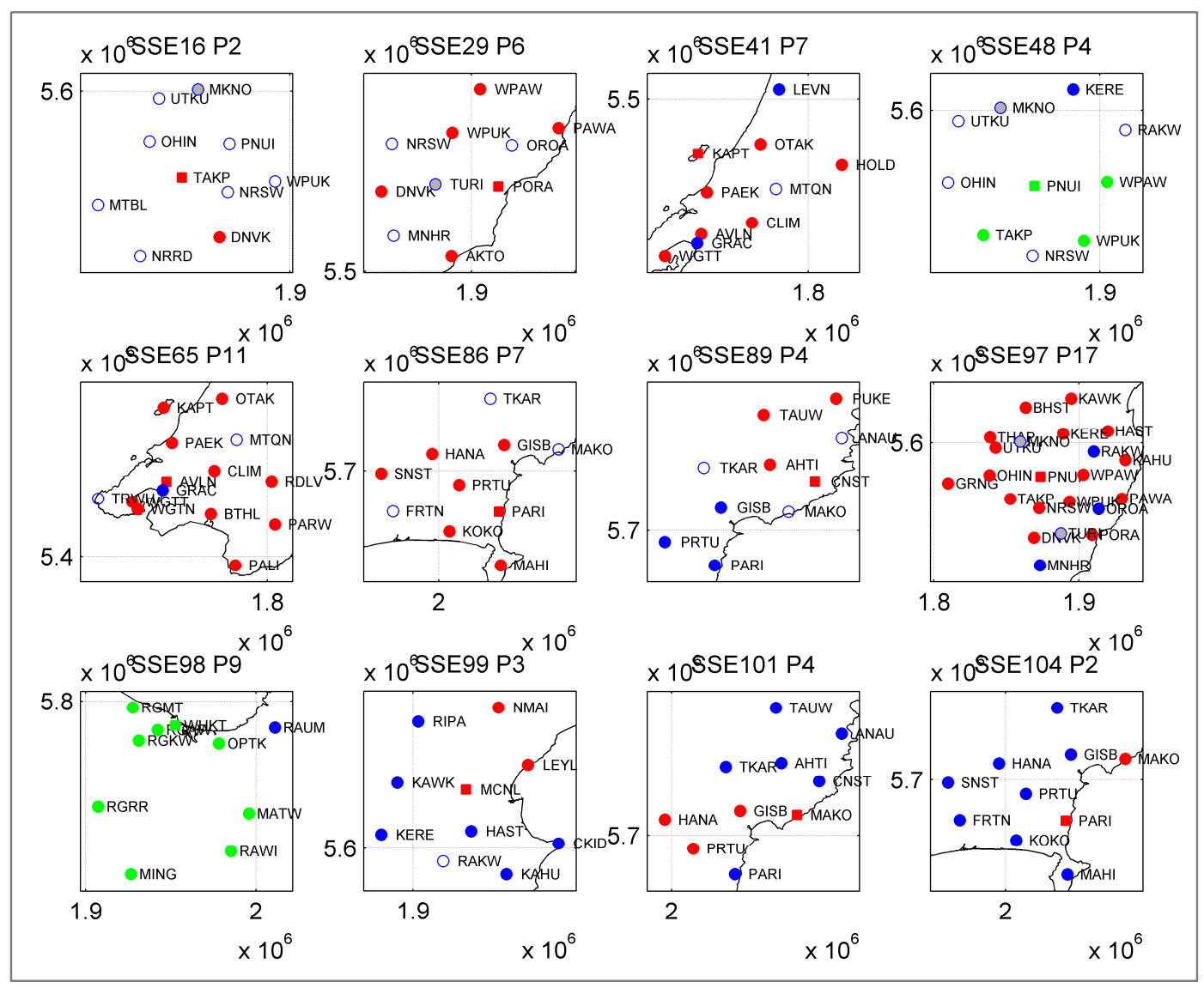

Figure 2.4.3: An example of a 'minimap' array used to find anomalies or sites that may have been missed for specific events. Red dots indicate sites at which the event has been detected; blue dots are sites that were active at the time but where the specific event has not been detected; green dots refer to the detection of "negative" events (see 2.4.5); and grey dots indicate sites at which no SSEs have been detected. Empty circles show cGPS sites that were not active at the time of the event, and red squares indicate the sites at which the surface displacement was largest for the specific event. Coordinates are in the NZTM2000 projection [LINZ, 2000]. Each map title includes the event number and the number of sites picked.

In practice, the index of events was copied to an Excel spreadsheet, so that renumbering of source events could be carried out expeditiously. A number of iterations were required, because, particularly for the smaller or slower events, the commencement date was often not particularly clear, and re-assignment of events to an alternative grouping may have been considered necessary. Re-assignment may have had to be carried out if, for example, multiple events occurred in close proximity in both time and space, as was commonly observed along the NE coast area. In some cases, it appeared that the source may have been moving, and a decision would have had to have been made as to whether to treat such a situation as a single or multiple source event. Generally, if start times 
could be more tightly grouped by dividing apparently similar or adjacent events in this way, then this was the approach taken.

\subsubsection{Audit}

Events that were detected at 4 or more sites were audited using Matlab code, designed by Prof. E. Smith, entitled 'SSE_cuts'. This code plots all the individual site picks for each event ('cut' from the traces) on a common basis and attempts to fit both an exponential and a logarithmic function to the trace - this was done purely to facilitate stacking and to allow visual comparison rather than for modeling (see below, section 2.5). Root mean square residuals contours are then plotted against time and scaling factor so that the quality of the fit for $\mathrm{c}$ and $\mathrm{t}_{0}$ can be assessed, where $c$ is a scaling factor and $t_{0}$ is the reference time in either of the following models:

$$
\begin{aligned}
& \text { either: } \quad d=\log \left(1+\frac{t-t_{0}}{c}\right)
\end{aligned}
$$

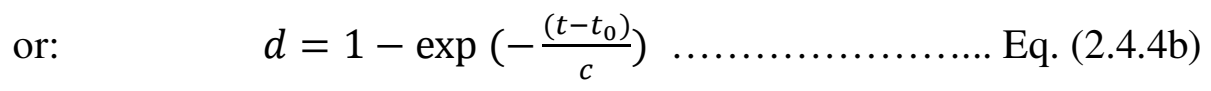

Fig. 2.4.4a illustrates an example of the use of 'cuts' for event 104, which was identified at 4 sites, and Fig. 2.4.4b illustrates the use of the corresponding error plotting. In this event, $\boldsymbol{t}_{\boldsymbol{0}}$ is well constrained in both models, but $\boldsymbol{c}$ is less well constrained for the logarithmic model. 

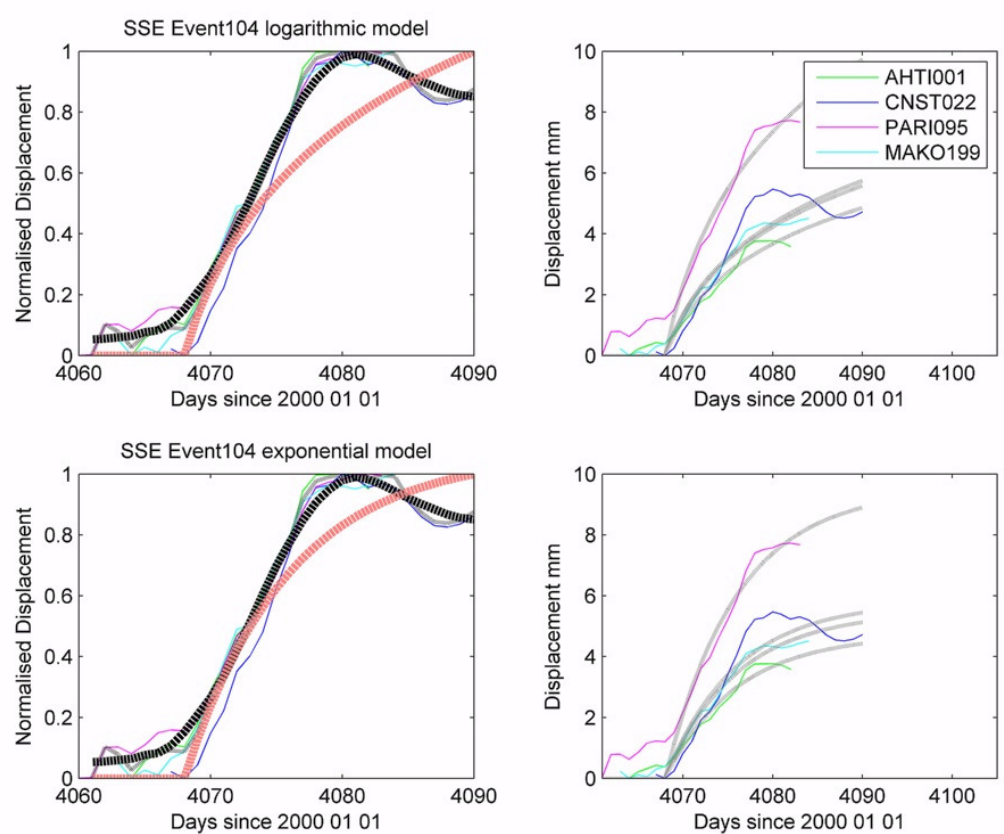

Figure 2.4.4a: Extracted 'cuts' from site records AHTI, CNST, PARI and MAKO for event 104, showing the absolute magnitude of the displacements on the right, with the best fit models for each trace in grey; and normalized displacements on the left, together with the stacked mean (black) and the best fit model for the mean (red).

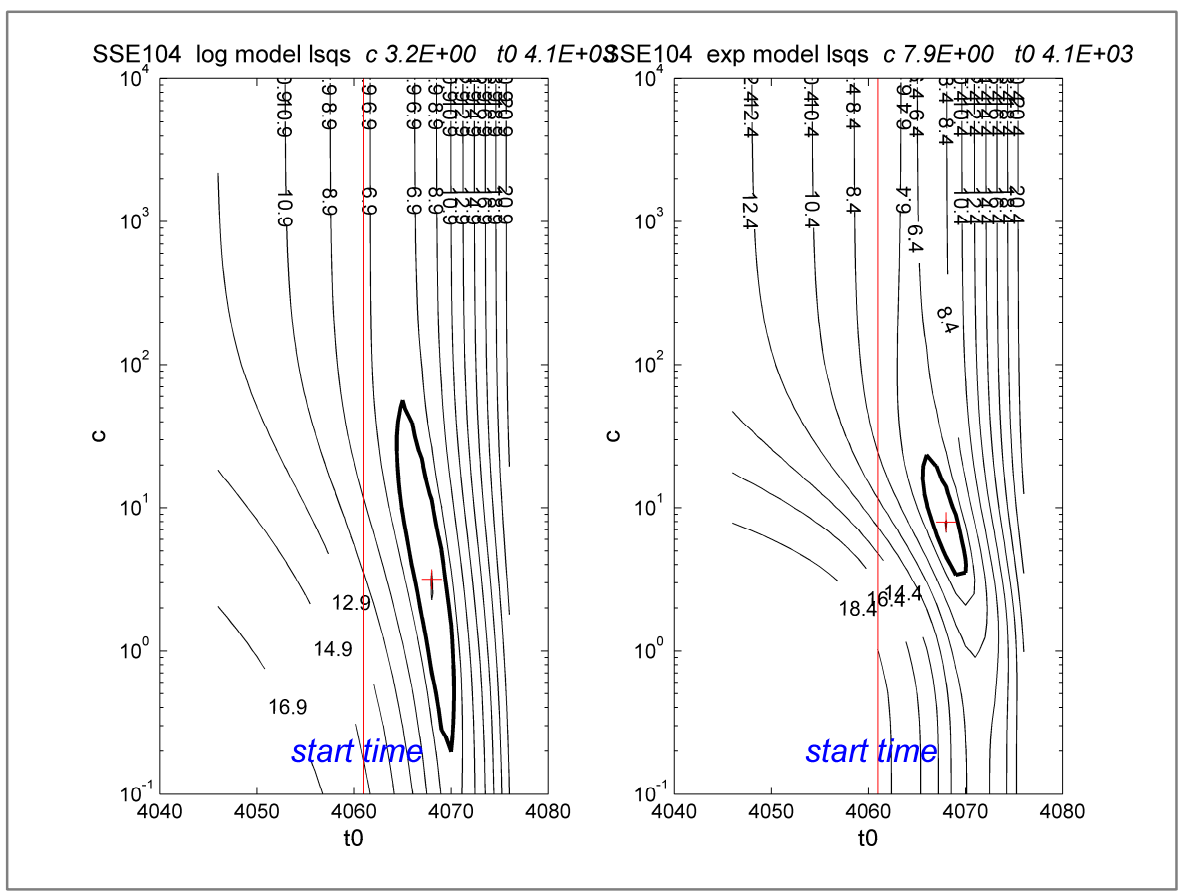

Figure 2.4.4b: Error plot for the data shown in Fig. 2.4.4a, illustrating the picked earliest start time (vertical red trace), the best estimate of the values for $\boldsymbol{t}_{\boldsymbol{0}}$ and $\boldsymbol{c}$ (red cross), and the rms contours for the fit to each model ( $\log$ on left, exp on right). The bold contour indicates the $95 \%$ confidence level. 


\subsubsection{Surface deformation 'event' categorisation}

Use of "SSE_cuts" allowed the "quality" of the data for each event to be assessed subjectively:

- Category 1: Events recorded at 4 or more sites for which $t_{0}$ and $c$ were wellconstrained in the error plots

- Category 2: Events recorded at less than 4 sites, but which had clearly-defined shapes on the displacement-time plots, or events recorded at 4 or more sites for which either $\mathrm{t}_{0}$ or $\mathrm{c}$ was poorly-constrained in the error plots

- Category 3: Events where the total displacement was less than $4 \mathrm{~mm}$ at all sites

- Category 4: Events observed at only 1 or 2 sites, with low amplitude and poor signal to noise ratio

- Negatives: Events that showed displacement in the opposite direction to that expected - i.e. westwards rather than eastwards on the east-component trace.

\subsection{Temporal evolution: deformation-time shape analysis}

One of the aims of the project is to give recommendations for automated or semiautomated detection of SSEs. One option under consideration required characterization of the "typical" shape of an SSE in the displacement-time domain. In the GNS Science papers it has been documented that the SSEs were temporarily characterised by a ramp function of variable duration, which comprised a linear ramp from 0 to 1 over a number of days [Beavan et al., 2008]. Wallace and Beavan [2010] subsequently describe an alternative method, which appears to require subjective assessment of a smoothing factor, followed by final assessment of start and finish times by eye.

It was initially considered that either a logarithmic or an exponential fit, as mentioned in 2.4.4 above, would be adequate. Assessment of the fits produced in "SSE_cuts" indicated that when a reasonable fit could be achieved, there wasn't much to choose between these two functions. As more data was analysed, it became clear that in some cases there appeared to be a precursory displacement (see Fig. 2.4.4a as an example) that was not fitted adequately by either option, and that the initial curvature was usually different to the final curvature. 
Both stacked "cuts" and individual site displacement-time "cuts" were examined using curve-fitting software [Systat, 2012]. This software treats the data in a purely statistical manner and can examine many options, but the range of equation types was constrained to "transition" or growth functions. This generic class of curves allows inclusion of the 5 "birth and death" stages: birth, growth, maturity, decline and death. Birth and Death Processes (BDP) are an important sub-class of Markov chains with continuous time parameter space, where the state space is the non-negative integers - these processes are characterised by the property that if a transition occurs, then this transition leads to a neighbouring state [Parthasarathy, 2007]. There have been many attempts in the literature to find simplistic forms that can be used for biological processes [Birch, 1999; Yin et al., 2003]. A number of growth curves are compared in Karkach [2006].

Whilst Larson et al. [2004] also acknowledged the curved start and finish aspects of the temporal development of the Guerrero SSEs, by use of a tanh function, this is symmetric in shape. The asymmetric sigmoid model seemed visually to be a better fit to the stacked mean curve shown in Fig. 2.4.4a, and has the essential characteristics of allowing different curvature at the beginning and end of the process. It is a special case of a growth curve based on the logistic distribution [Malik, 2006]. The rate of growth depends on the multiplicative interaction between the accelerating and decelerating parts of the function:

$$
\frac{d F}{d x}=c[F(x)-A][B-F(x)]
$$

where: $\boldsymbol{c}, \boldsymbol{A}$ and $\boldsymbol{B}$ are constants, with $\boldsymbol{c}>0$, and $\boldsymbol{B}>\boldsymbol{A}$

Growth curves of this type are commonly used in population studies and econometrics as well as bioassay and chemical reaction dynamics. Logistic functions of one type or another are commonly used for modeling the evolution of a wide range of systems over time [Höök et al., 2011; Kucharavy and De Guio, 2011]. The lognormal distribution is similar to the logistic distribution. The cumulative lognormal is asymmetric sigmoidal in shape. Wherever there are effects that act in a multiplicative manner (as, for example in equation (2.5) above) the result is a lognormal distribution [Limpert et al., 2001].

Hence the subsequent curve-fitting exercise was primarily focused on asymmetric sigmoids. Further details are given in 3.2.5. 


\subsection{Inversion}

The key elements involved in inversion of surface movements to estimate source displacements are:

1. A model of the source fault or fault zone

2. Basis functions describing the elastic behaviour of the affected subsurface volume

3. A search and smoothing algorithm

\subsubsection{The Hikurangi subduction interface model}

In all the work on SSEs along the Hikurangi subduction zone to date, it has been assumed that the source displacement takes place over an area on the subduction interface, or close to it.

The Hikurangi subduction interface has been modeled by Ansell and Bannister [1996]. This model has been updated by $C A$ Williams et al. [2013], and is depicted in Fig. 3 in their paper, reproduced as Fig. 2.6.1a below.

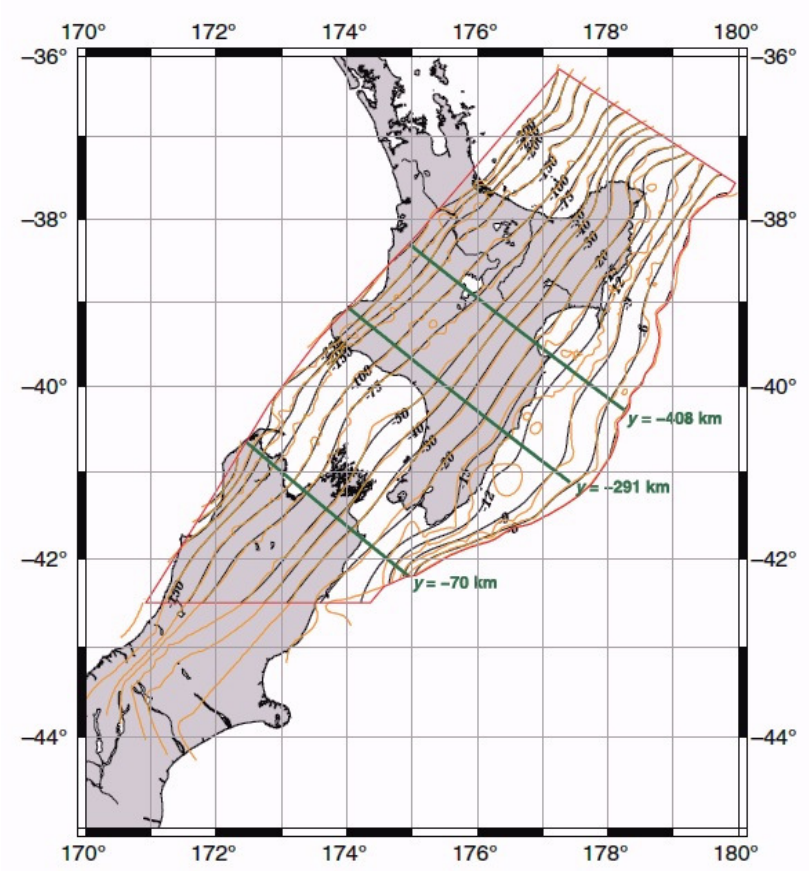

Figure 2.6.1a: (reproduced from C A Williams et al. [2013]), Hikurangi subduction interface model, with orange lines indicating interface contours predicted by radial basis function interpolation, and black lines indicating the bi-cubic-spline-smoothed contours. 
Lamb and Smith [2013] used cGPS data and Okada's code to model the hanging wall on the Hikurangi subduction zone as a continuous elastic block (as compared with the multiple block models used by other authors). They commenced with a model of the subduction megathrust defined by the top of the region of deep intensive microseismicity in the Benioff zone, which, as they state, is well-constrained by tens of thousands of microearthquakes recorded in the GeoNet catalogue over the past 30 years [Reyners et al., 2011].

Lamb \& Smith's model is encoded in a Matlab routine 'Benioff.m', which allows an interface model to be extracted from the catalogue data using pre-defined statistical techniques. Fig. 2.6.1b illustrates the pseudo 3D distribution of detected earthquakes, and fig. 2.6.1c shows an example of one slice through the model at 525000 northing on the rotated transverse NZ Mercator projection (NZTM2000 [LINZ, 2000]). (This projection is rotated to run approximately parallel to the Hikurangi trench offshore of the North Island.)

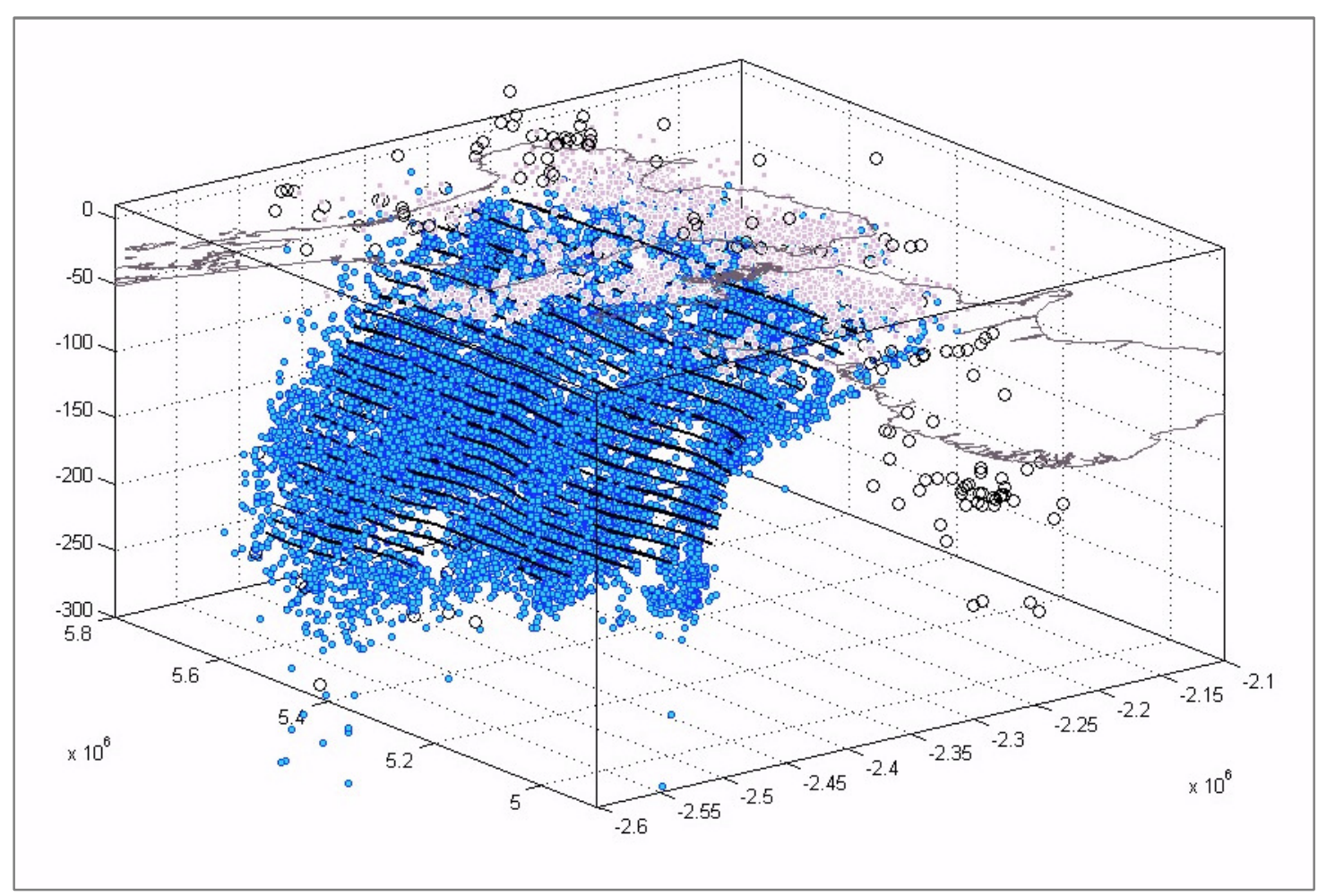

Figure 2.6.1b: Pseudo-3D visualization of the seismicity in the Benioff zone of the Hikurangi subduction. Black lines indicate the position of contours at $10 \mathrm{~km}$ depth increments. Events shallower than $30 \mathrm{~km}$ are shown in grey, for clarity. 


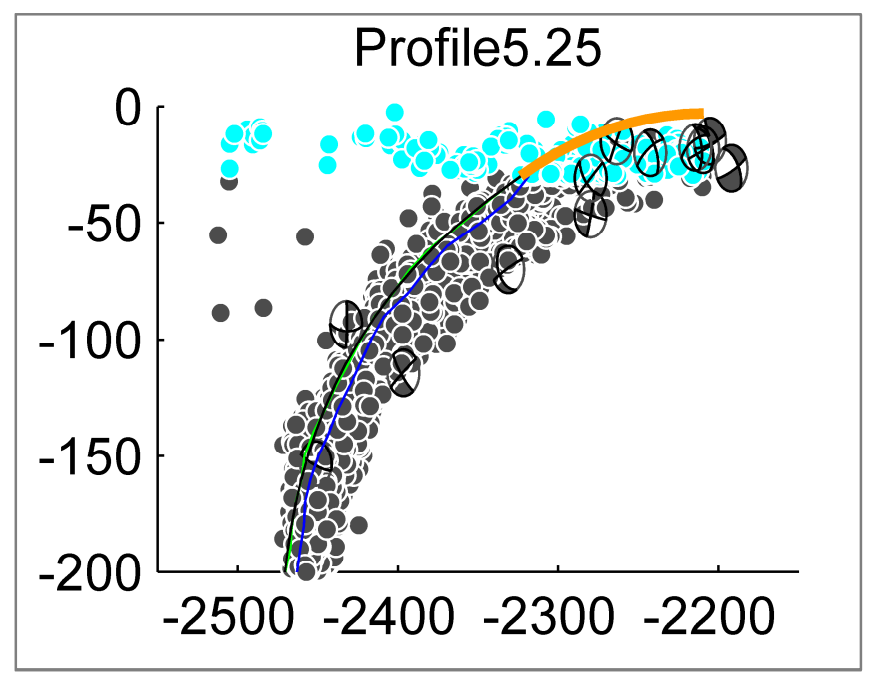

Figure 2.6.1c: Example cross-section down to $200 \mathrm{~km}$ along northing 525000 (located approximately along the line Kapiti to Martinborough) on the rotated NZTM projection of the Benioff zone. Individual seismic events are shown as closed circles, with seismicity above $30 \mathrm{~km}$ depth in cyan, and moment tensor 'beachballs' shown where available. The median of the distribution (ignoring events more than $20 \mathrm{~km}$ from the Australian side of the Benioff zone) is shown as a blue line; the green line indicates the upper $7.5^{\text {th }}$ percentile of the distribution; the black line is a $4^{\text {th }}$ order polynomial fitted to that surface; and the orange line is a circular arc matched in radius to the upper part of the polynomial and terminating at the Hikurangi trench.

The Williams and Lamb and Smith interface models are compared in Appendix 6.

It may be noted in passing that due to smoothing, neither of the models appear to have sufficient resolution to detail the Taranaki-Ruapehu discontinuity described by Salmon et al. [2011].

\subsubsection{Elastic response to source displacements - Inversion}

In New Zealand, the GNS-based teams [Douglas et al., 2005; McCaffrey et al., 2008; Wallace and Beavan, 2006; 2010; Wallace and Eberhart-Phillips, 2013; Wallace et al., 2012a], have, in the past, largely used McCaffrey's 'DEFNODE' code [McCaffrey, 2007], or 'disloc99' [Darby and Beavan, 2001]. 'DEFNODE' uses the Okada dislocation functions, whereas 'disloc99' uses derivations by Masinha and Smylie [1971], both of which can be traced back to work by Steketee [1958] on the Volterra formula [Volterra, 1907]. Until 2013 the GNS team modeled 3-parameter Gaussian processes on sections, but in the more recent papers this technique was abandoned in 
favour of inversion directly onto the nodes. The SSE source events are all assumed to be located on the subduction interface, and the fault strike is assumed to follow the strike of the margin. In the cases where ' $D E F N O D E$ ' has been used, the relative motions on the interface have been assumed to be parallel to the long-term relative motion derived from the Wallace et al. [2004] block models, whereas where 'disloc99' has been used, the rake has been unconstrained [Wallace and Beavan, 2010].

In their elastic modeling of the subduction zone, Lamb and Smith [2013], for their 2D sections, used Fortran code based on an elastic half-space in 2D, calling on the dislocation functions of Okada [1985; 1992].

Procedures for calculating elastic displacements resulting from point and discrete sources, both at the surface and at depth, have been outlined by a number of authors (see Appendix 5), but work by Okada is the most commonly referenced [Okada, 1985; 1992] and forms the basis of a number of inversion codes. In this method, displacements are calculated from discontinuities embedded within an homogenous elastic half-space.

Inversion procedures written in Matlab by Prof. E. Smith also use the Okada method. These have been validated using the original Fortran code published by Okada (see Appendix 7). The primary process used in the code is as follows:

i. The subduction interface model is discretized into $5 \times 5 \mathrm{~km}$ segments (as measured on the surface projection).

ii. For each segment, sets of Basis functions are calculated (based on Okada's DC3D) for each displacement component separately for $1 \mathrm{~m}$ slip in the dip-slip (ds) and strike-slip (ss) direction. A database is then generated of the 6 elastic responses ( 3 components $\mathrm{x}$ ds and ss) at each cGPS location for each segment. Thus the rake of the slip is not constrained and the inversion code calculates for both dip-slip and strike-slip components.

iii. For each surface 'event', the user seeks a least-squares fit to the observed cGPS data (weighted inversely by the SSE-free rms values), initially starting with a specified number of source segments centred on the site with the largest surface displacement. The source configuration is the spiralled away from its starting point by a specified number of fine grid steps, with the RMS misfit being calculated at each gridpoint. The gridpoint with the minimum RMS fit defines the trial centre of the slip. In some cases more than one minimum is identified, and user intervention may then be required to decide the minimum that best fits the expected location pattern. In most cases the one automatically chosen by the algorithm could not be improved upon.

iv. The code then allows the area of the slip and the number of spiral steps to be varied and the user seeks to find a centre and fault area that minimises overall the RMS residuals. 
In general terms, whilst the fit is sensitive to event moment, it is not very sensitive to modelled slip area, and hence slip areas have been adjusted to produce slip components of less than an arbitrarily chosen value of approximately $30 \mathrm{~cm}$. As at Feb 2015 the code was limited to slip patches that are square on the surface projection.

v. The code is capable of dealing with observations from any number of sites, but clearly, the uncertainties increase for events that were only detected at a limited number of sites (or even 1 site). No events have been deleted - users can assess the reliability of each event based on the confidence limits. Sites that might have been expected to have contributed to a solution, but show no evidence of an event, are included with null values. In order to constrain the location of source slips in which observations are limited to 1 or 2 sites, the minimum number of null sites is 3 , otherwise available null sites are added on a radially-expanding basis, up to a maximum equalling the number of sites at which there are event observations. Data from sites remote enough from the source to be expected to have zero measurable displacement do not provide independent data, and hence one degree of freedom is counted for each null site, in contrast to $3 \mathrm{DoF}$ for the non-null sites (one for each component).

vi. For each source event, the code produces a map (in rotated NZTM coordinates) that shows the modelled and observed horizontal and vertical cGPS displacements with their uncertainties, together with the estimated source location, direction and amount of slip. Each map is accompanied by a contour plot of the RMS residuals from the inversion, which allows the quality of the fit to be visually assessed. An example is shown in Fig. 2.6.2.

vii. The main characteristics of each SSE or source event are summarised in the catalogue, together with the estimated uncertainties in each parameter. Uncertainties in the moments are estimated by Monte Carlo methods (with $\mathrm{N}=500$ ) using the appropriate uncertainties in site displacements shown in Appendix 11 as the primary source of variance. In the case of the source location, the $95 \%$ confidence limits are based on the RMS residual value plot, and can only be established where these form a closed contour within the search area - one exception is calculated for events where the search area would include slip patches east of the trench, where the confidence interval contour would otherwise be expected to close.

viii. The equivalent seismic moment $\mathrm{M}_{0}$ is calculated using a rigidity value of $40 \mathrm{GPa}$ (to be consistent with the calculations produced by GNS):

$\mathrm{M}_{0}=$ rigidity $\mathrm{x}$ area $\mathrm{x}$ slip

where slip $=$ sqrt $\left(\mathrm{ds}^{2}+\mathrm{ss}^{2}\right)$, and ds and ss are slip components in the dip and strike direction respectively

$\mathrm{M}_{\mathrm{W}}$ is calculated using Kanamori's formula [Kanamori, 1977; Kanamori and Anderson, 1975]:

$$
\mathrm{M}_{\mathrm{w}}=(2 / 3) * \log \mathrm{M}_{0}-6.05
$$

ix. Residuals and weights for each component at each cGPS site are written back to file for further analysis. 


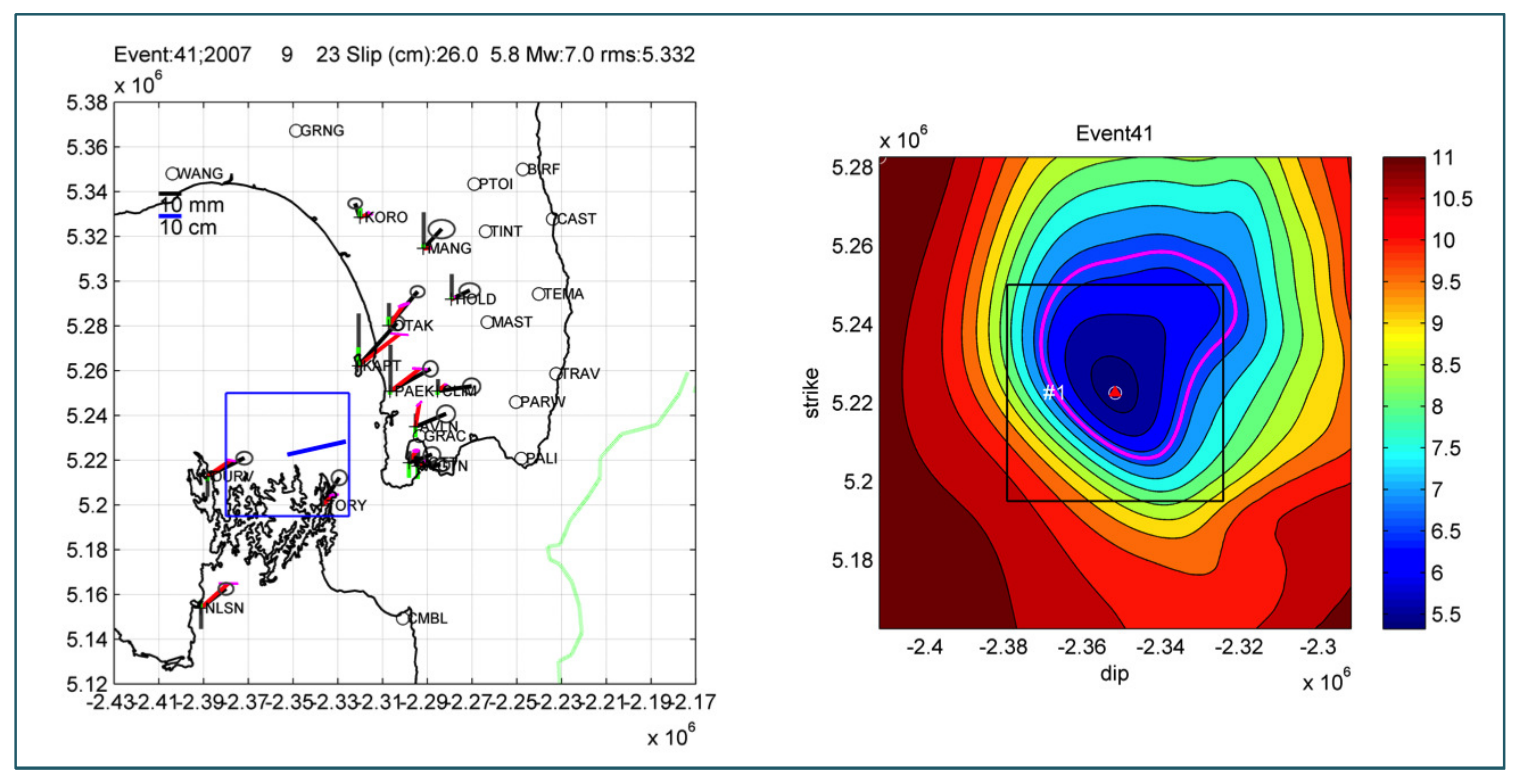

Figure 2.6.2: An example of an SSE inversion plot showing the modeled (red) and observed (black) horizontal cGPS displacements and their confidence ellipses, the modeled and observed vertical displacements (grey and green), and the estimated source area and slip; together with the RMS residuals contours from the inversion process. Open circles on the map indicate cGPS sites at which the event was not observed ("nulls") and which were included in the inversion. The magenta contour on the residuals plot indicates the $95 \%$ probability region for the source slip location. The coordinate system is rotated NZTM.

\subsubsection{Catalogue analysis}

Detailed analysis of the catalogue was not part of this project. Preliminary statistics are, however, discussed in chapter 5.

\subsubsection{Effects of layering}

In an analysis of the 1966 Parkfield earthquake, Rybicki [1971] indicated that a simple half-space homogenous elastic model is inadequate to describe far-field displacements. It was stated that in the case of a buried strike slip fault with a softer layer overlying the source, calculations based on an homogenous half-space would tend to under-estimate the depth of the fault. This was again noted by J C Savage [1987].

Whilst acknowledging this issue, Okada [1985] considered that the homogenous halfspace model was adequate for inversion purposes since it was a good first approximation, the source model was inherently non-unique, and the quality of crustal elastic data was often generally poor. 
In analyzing the 1989 Lomo Prieta earthquake Eberhart-Phillips and Stuart [1992] carried out finite element analyses using data from detailed active seismic exploration to try and explain the apparent anomaly between source locations estimated by geodetic data and by seismology. They showed that the geodetic slip locations from the FE analysis would be offset in both depth and location from previously estimated locations based on homogenous half-space assumptions, and hence closer to the San Andreas fault.

J C Savage [1998] examined the effect of layering on surface displacements from a dipslip fault, as did Cattin et al. [1999]. Subsequently Wald and Graves [2001], in their modeling of the Northridge earthquake fault geometry, bluntly concluded that "the commonly-used half-space approximation is inadequate".

Hearn and Burgmann [2005]; $R$ Wang et al. [2003] both examined the effect of layering on analyses of the 1999 Izmit earthquake and came to similar conclusions. Montgomery-Brown et al. [2009] found that SSEs assumed to be on a shallow dipping thrust fault on the south flank of Kilauea, inverted using uniform half-space, gave depths significantly at variance with associated seismicity locations. In analyzing coseismic GPS data for sedimentary basins in China, Chen et al. [2011] concluded that by ignoring layering and using simple half-space models for inversion could result in seismic moments being over-estimated by up to a factor of two. A similar magnitude of error was suggested by Masterlark [2003] after assessing 6 finite element models of coseismic displacement from the $1995 \mathrm{M}_{\mathrm{w}}$ 8.0 Jalisco-Colima, Mexico, subduction zone earthquake and associated measurements from a nearby GPS array. Cavalié et al. [2013]; Radiguet et al. [2012] used layering in their inversion models for analysis of both GPS and InSAR data on long-period slip on the Guerrero Gap 2006 SSE. Sato et al. [2007] carried out extensive three-dimensional modeling of displacements arising from slip on buried faults offshore northern Japan and concluded that ignoring inhomogeneity could lead to errors of up to $40 \%$ in source slip inversion results.

The subject of the effect of layering on displacement fields from buried faults occupies an entire chapter in Segall [2010], and it seemed clear to this author that the apparent potential magnitudes of the effects on estimates of moments (of the order of up to tens of percent) could not be ignored. 
The relevance of this issue hinges on whether there are significant differences in the stiffness profiles between the various GPS sites and the detected SSEs along the Hikurangi margin. Profiles perpendicular to the margin have been published for various locations.

Seismic velocity $\mathrm{V}_{\mathrm{P}}$ is probably the simplest proxy for stiffness, for which there are significant published data. An early summary is shown in E G C Smith et al. [1989]. Reyners et al. [2006] produced $\mathrm{V}_{\mathrm{P}}$ and $\mathrm{V}_{\mathrm{P}} / \mathrm{V}_{\mathrm{S}}$ profiles for the central North Island at approximately $30 \mathrm{~km}$ intervals along strike from just NE of the Mahia peninsula to just NE of the line running through Wanganui. One segment of their Fig. 6 (reproduced as Fig 2.6.4a) illustrates $V_{P}$ variability at $11 \mathrm{~km}$ depth over dip and strike distances $(X$ and $\mathrm{Y}$ in the figure) of 275 by $275 \mathrm{~km}$.

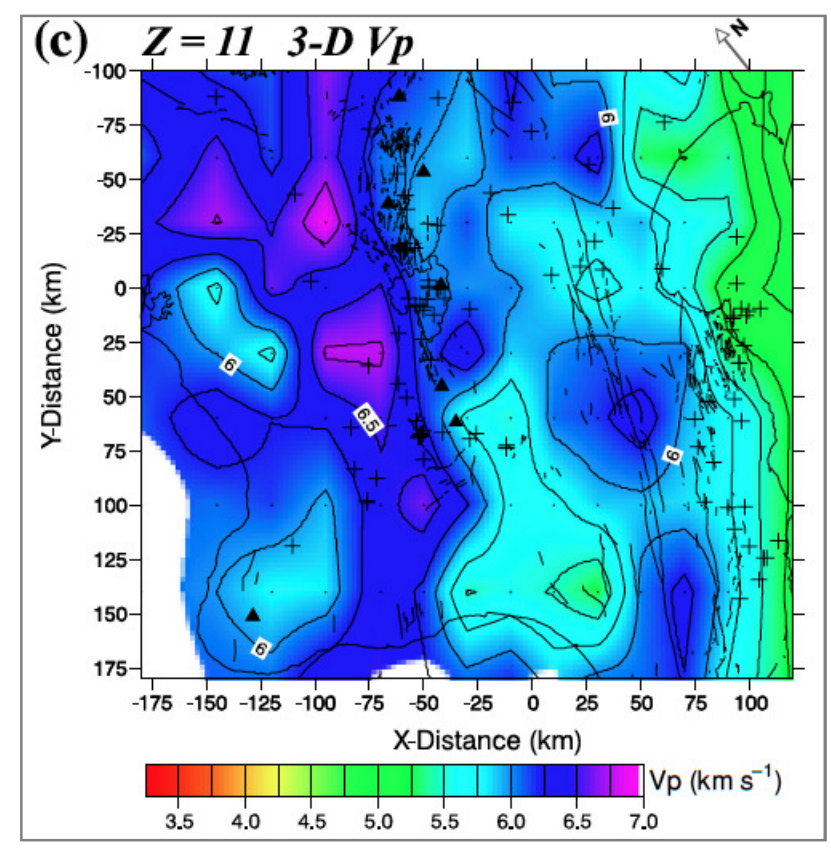

Figure 2.6.4a (reproduced from Reyners et al. [2006]): Variation in seismic velocity $\mathrm{V}_{\mathrm{P}}$ at $11 \mathrm{~km}$ depth in the central part of the North Island. Mount Taranaki is indicated by the black triangle lower left, and Mahia Peninsula is outlined at top right.

The most northerly profile in the literature is that of Bassett et al. [2010], situated approximately $50 \mathrm{~km}$ offshore of the Raukumara peninsula. As reproduced in Fig. 2.6.4b, this indicates significant layering above the subduction interface. 


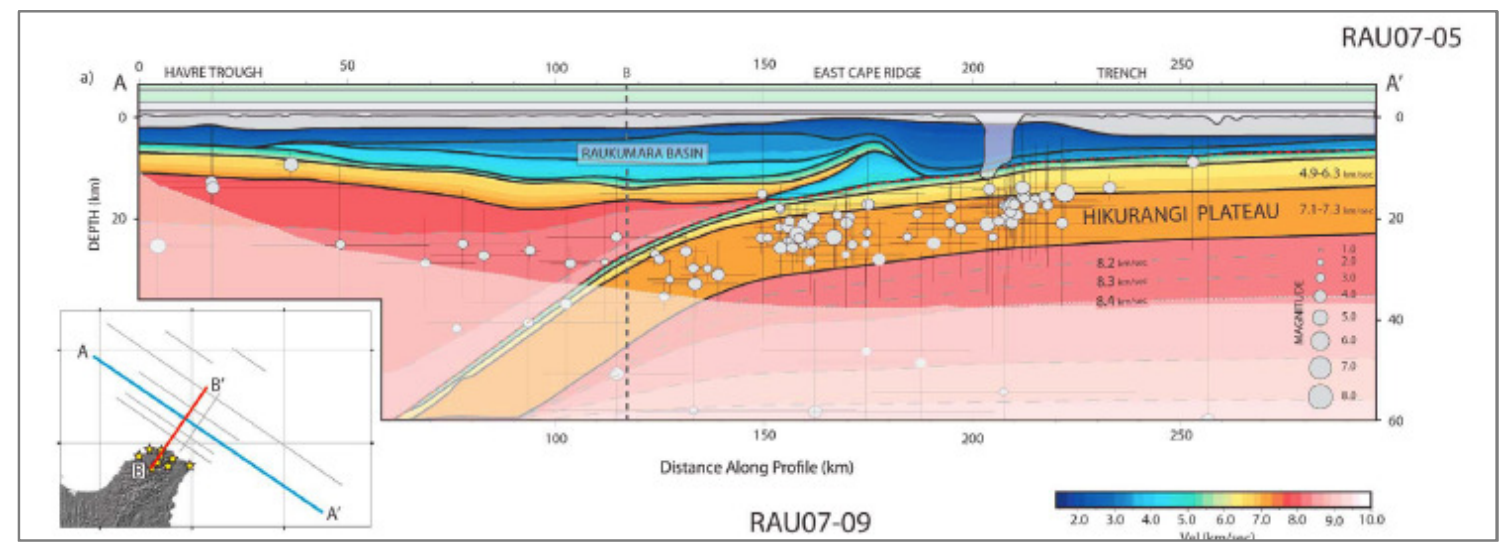

Figure 2.6.4b (reproduced from part of Fig. 6 of Bassett et al. [2010]): Cross-section along RAU07-05 (blue line, inset) displaying the dip parallel $\mathrm{V}_{\mathrm{P}}$ structure through their velocity forward model. Masked areas illustrate regions not sampled by seismic data. Earthquakes with $\mathrm{M}_{\mathrm{W}}>2$ and hypocentres within $5 \mathrm{~km}$ of the profile are plotted and scaled to magnitude. Earthquake locations were downloaded from GeoNet and not located using this velocity model.

These authors analysed the geometry and thickness of the southward continuation of a prismatic-shaped low velocity $(3.5$ to $5.0 \mathrm{~km} / \mathrm{s})$ anomaly, and indicated that the thickness appeared to vary significantly over this region. Davey and Ristau [2011] also discussed mantle seismicity below the Raukumara peninsula, and suggested that the crust is thin in the fore-arc region and underlain by altered mantle.

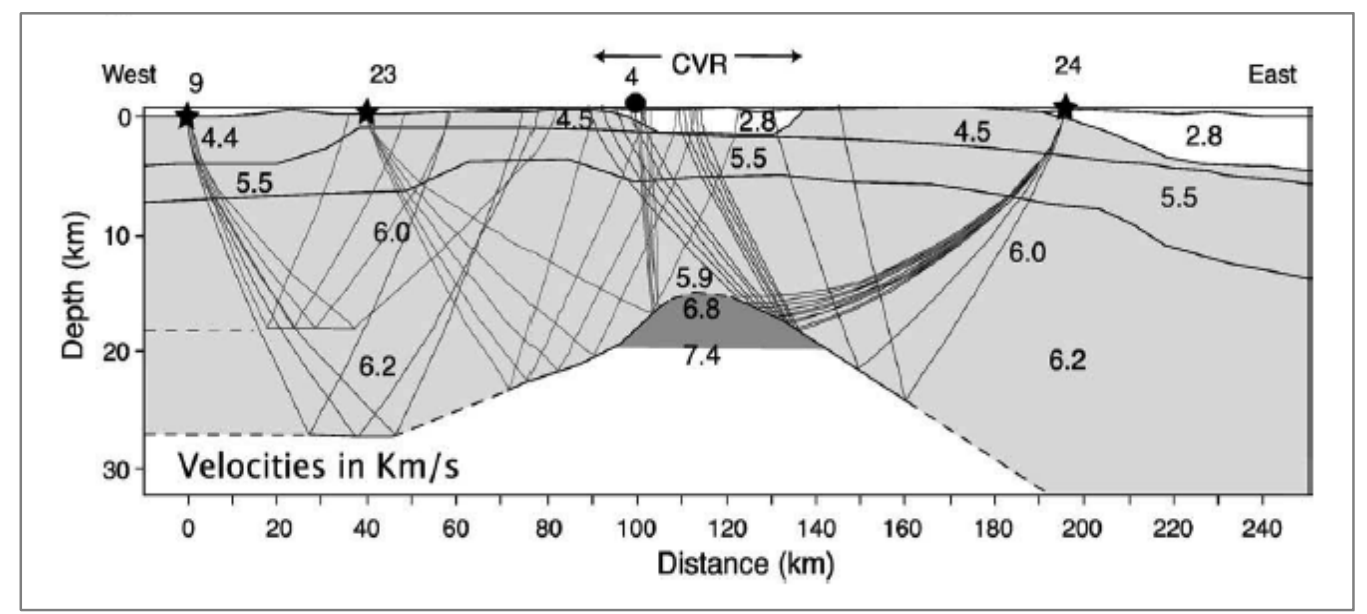

Figure 2.6.4c (reproduced from Fig. 6 of Stern et al. [2010]): Ray-tracing model for a series of seismic shots across the east-west line of the NIGHT project. Ray paths where the crust mantle boundary and lower crust are sampled from shots are shown. Other boundaries without rays on them were derived from refraction phases that are not shown. Dashed lines are inferred boundary continuations. Velocities are shown in $\mathrm{km} / \mathrm{s}$ and the velocities within the crust-mantle transition zone are taken from a north-south line. 
Towards the centre of the region with which we are concerned, Stern et al. [2010] also indicate a structure with significant velocity layering, with a substantial low velocity $(2.8 \mathrm{~km} / \mathrm{s})$ layer just offshore Hawkes Bay, as reproduced in Fig. 2.6.4c.

Whilst data from Taupo Volcanic Zone (TVZ) cGPS stations have currently been excluded from the analyses, it should be noted that significant velocity layering was also documented in this zone [Stern and Benson, 2011; Stern et al., 2010].

Further south, data from the SAHKE transect gave information closer to the Wellington-Kapiti area. This is covered by Henrys et al. [2013], but a pictorial schematic in Fig. 65 of C. Hall's MSc thesis [Hall, 2013] and reproduced as Fig. 2.6.4d illustrates this most clearly.

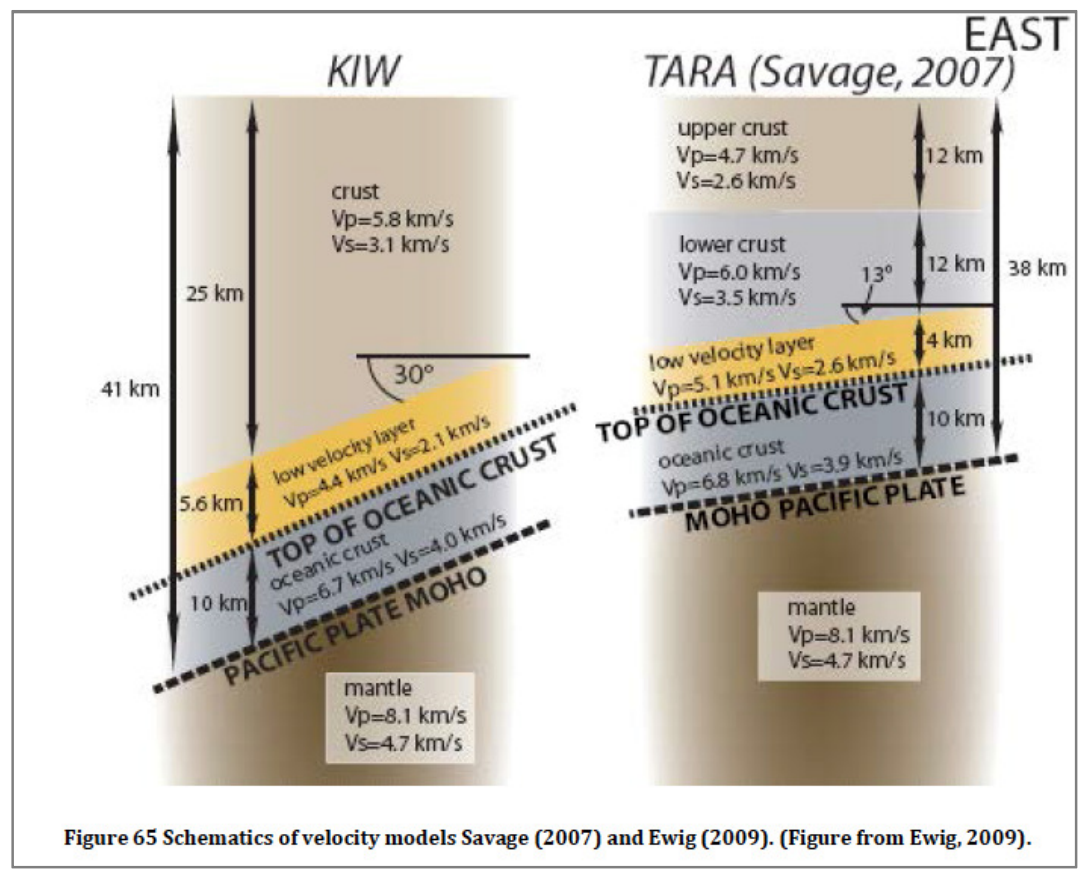

Figure 2.6.4d (reproduced from Fig 65 of Hall [2013]): Schematics of velocity models by $M$ K Savage et al. [2007] and Ewig [2009]

There thus appears to be adequate cause to investigate the subject further. The issue has been explored using modeling based on EDGRN and EDCMP code [ $R$ Wang et al., 2003], compiled under GNU Fortran, and is discussed in detail in Chapter 4. 



\subsection{Results}

\subsection{Noise reduction}

\subsubsection{Residual noise levels}

The effectiveness of the noise reduction can be judged in several ways. Figure 3.1.1a shows comparisons for all sites between the rms values of the residuals of linearly detrended series from both the raw daily solutions, and the final regionally-filtered locally de-seasoned series for the east component (note that the east component is most sensitive to SSEs along the Hikurangi margin).

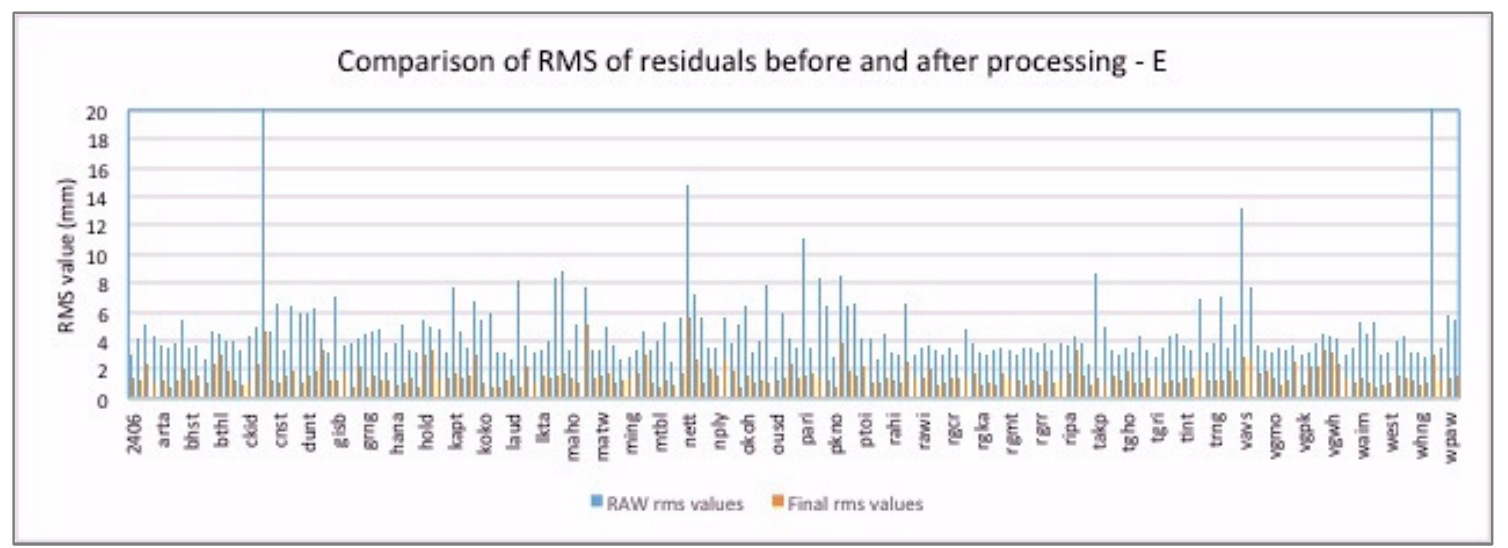

Figure 3.1.1a: Comparisons of rms values of linearly de-trended residuals for all sites on eastcomponent raw data (blue) and after noise processing (orange)

The average reduction in rms noise levels was 63 (sd 14)\%, 49 (sd 15)\% and 37 (sd $11) \%$ for the $\mathrm{E}, \mathrm{N}$ and $\mathrm{U}$ components respectively.

The spectral content of the data was also examined. Fig. 3.1.1b shows spectral density plotted against period (in days) for raw data from a range of sites. It can be seen that in the raw data, there are many spectral peaks that largely correspond with draconitic harmonics. The average period and amplitude of these peaks are summarized in Fig. 3.1.1c. 


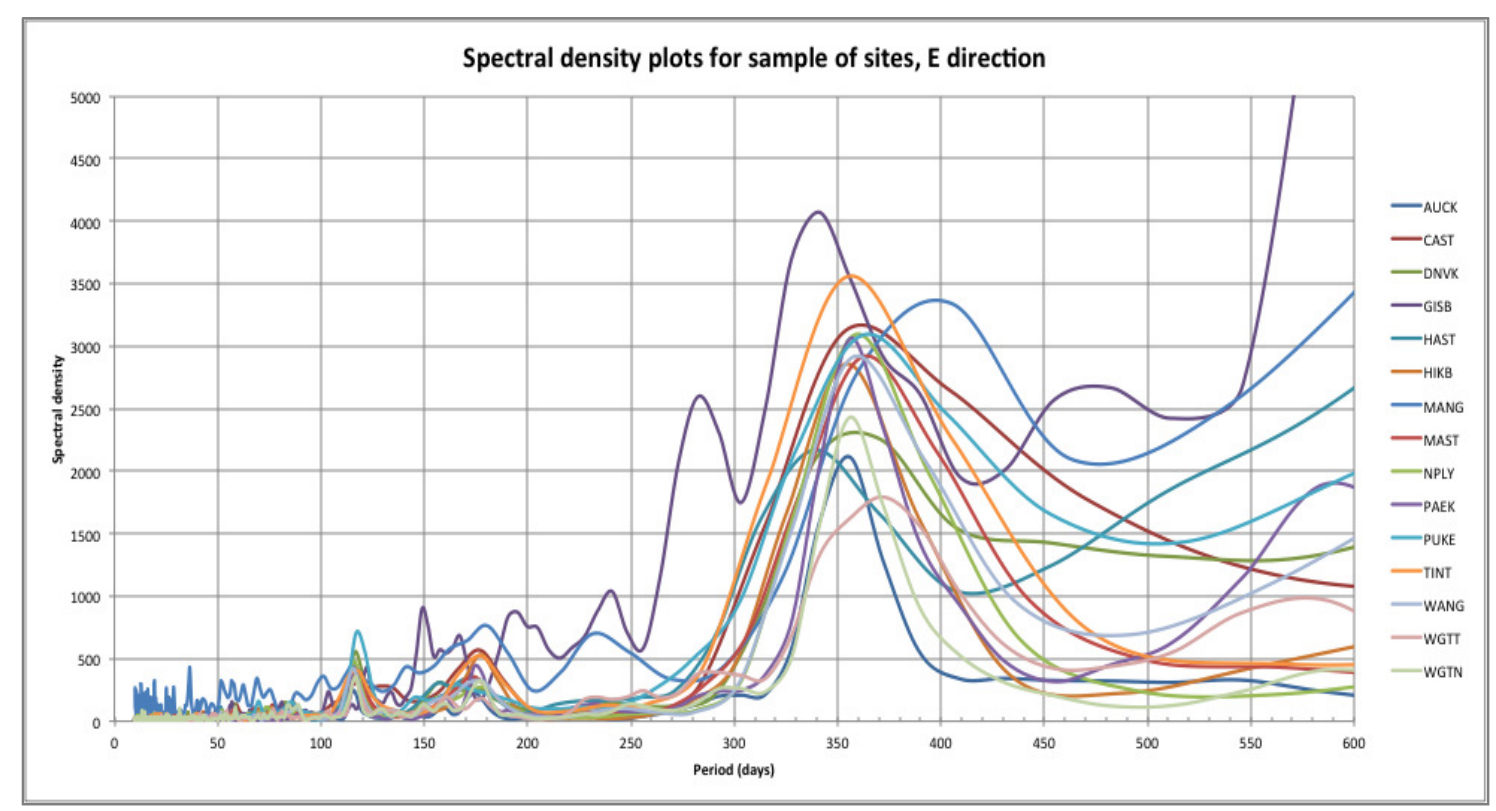

Figure 3.1.1b: Spectral density against period plots for raw cGPS data from a range of North Island sites

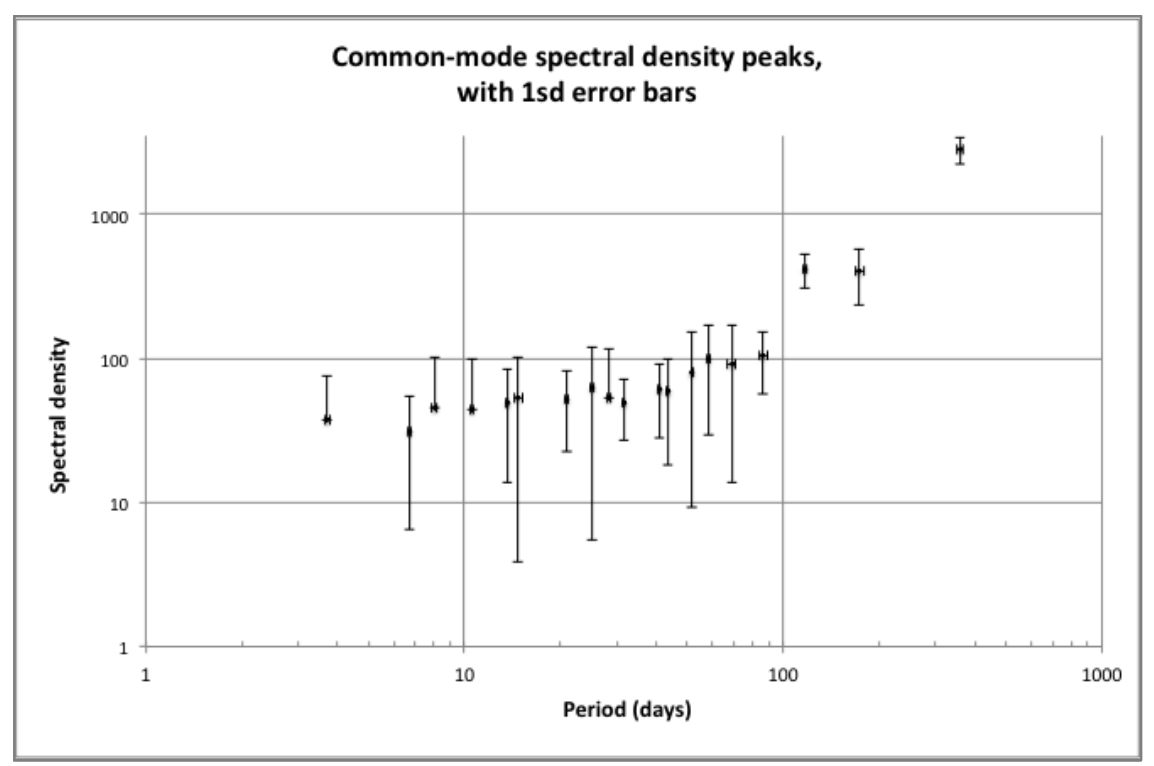

Figure 3.1.1c: Periods and 1 standard deviation uncertainties for spectral peaks appearing in typical raw cGPS series. Negative ordinate error bars that would extend below zero have not been plotted, thus highlighting points that may not be significantly different to zero spectral density at the $67 \%$ level.

It is observed that the noise processing completely changes the spectral characteristics. Fig. 3.1.1d shows the power spectral density plots for AUCK, E component, before and after noise processing. Note the different amplitude scales. It can be observed that the character changes from 'pink + white' (correlated + random) noise to 'blue + white' 
(random) noise. All of the flicker (correlated) or relatively low-frequency noise has been removed. The remaining noise is all relatively high-frequency and random.
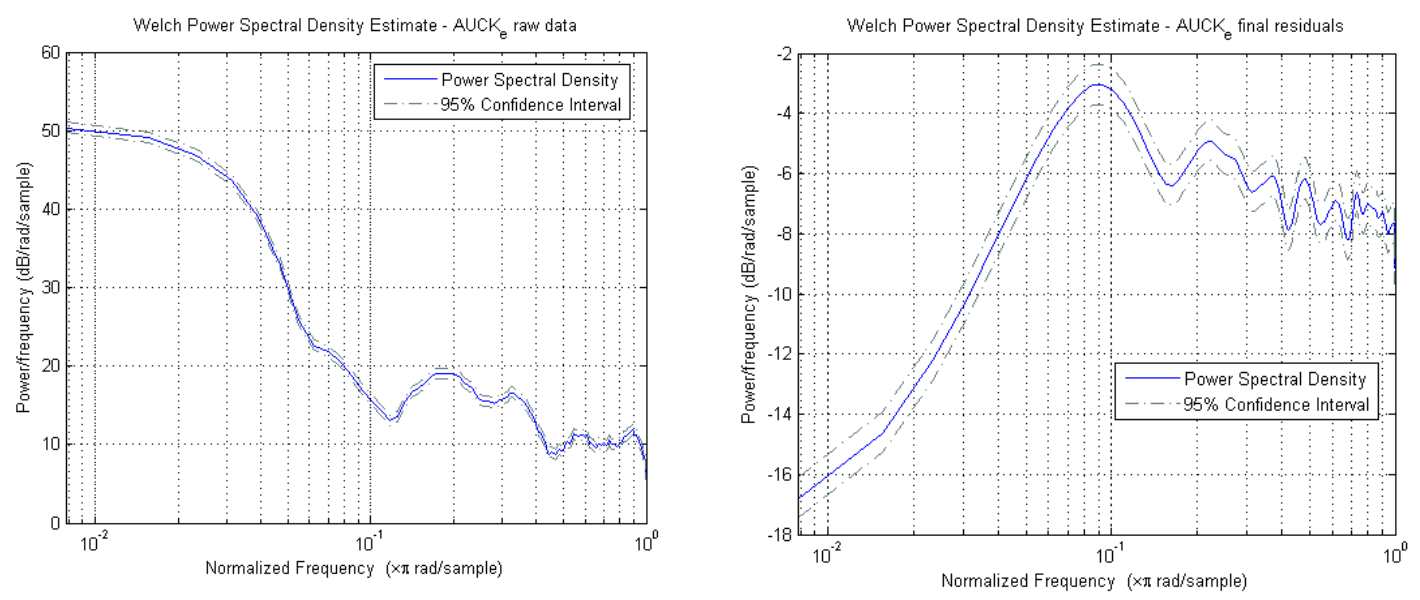

Figure 3.1.1d: Power spectral density functions for AUCK_e before (left) and after (right) noise processing - note the significantly different amplitude scales.

\subsubsection{Factors affecting residual noise}

It will be noted from Fig. 3.1.1a that even after noise reduction, some sites were still much noisier than others. A number of factors were investigated as potential contributory causes: monument type, antenna model, local subsoil conditions, geographic location and height above sea level.

Residual noise values were obtained from an output file, Velbitout.xls, in which the rms values of residuals from the SSE-free sections of de-trended data are tabulated for each site and component. (SSE-free sections are indicated on displacement-time plots by alternating azure and cyan horizontal lines, as, for example, shown in Figure 1.1)

The correlations between rms values for each component were examined - see Fig 3.1.2a. This indicates that, since the noise levels are highly correlated between the components, there was no reason to use one or another component to characterize the noise levels, and therefore the rms 'average' of 3 components has been used as the best estimate of overall noise levels at each site. 


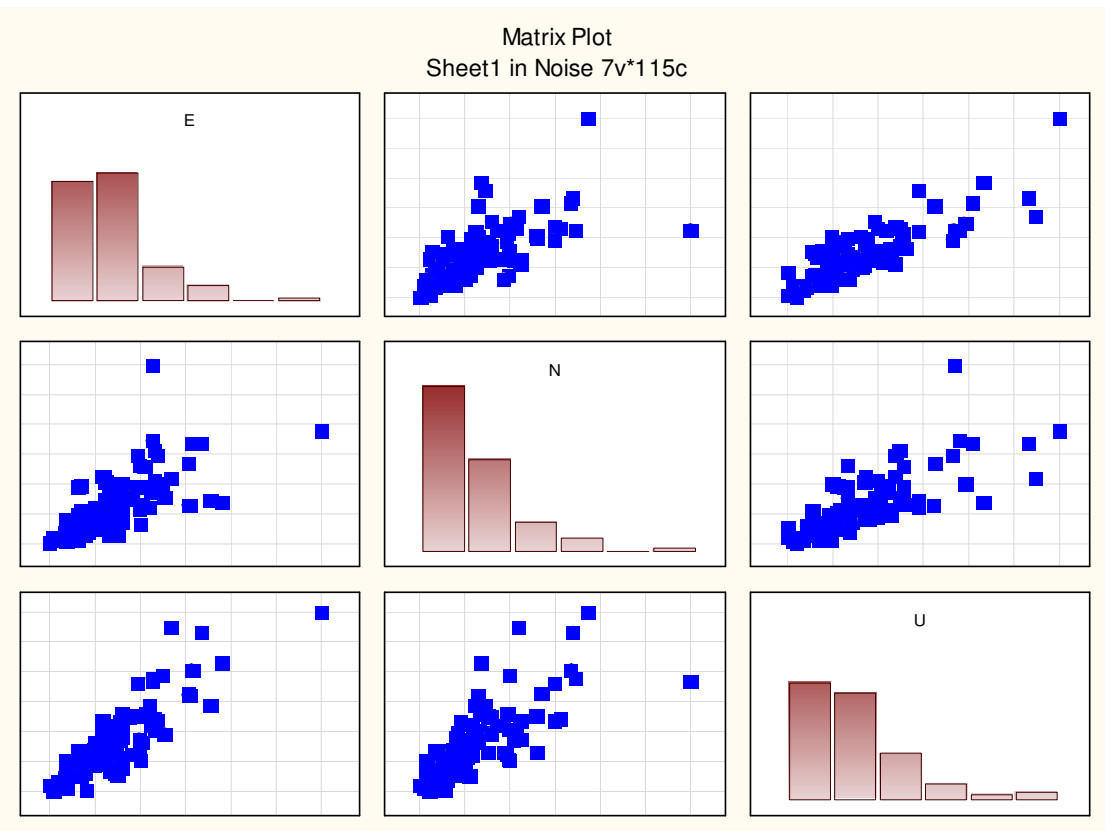

Figure 1a: Correlation matrix plot for rms noise levels for $\mathrm{E}, \mathrm{N}$ and $\mathrm{U}$ components for all cGPS sites. The diagonal histograms show the shapes of the distributions for each component. In each of the scatter plots, the abscissa is the component shown in the column, and the ordinate is the component shown in the row. Each plot is scaled to the maximum value in each component.

The influence of potential contributory causes listed above was examined using analysis of variance, with locations and elevations as covariates - see Appendix 8 . This suggested that only latitude and site elevation were significant covariates at the 0.01 level. It is unclear why latitude should be a significant variable, unless atmospheric water vapour is more variable in the southeast than in the northwest.

Fig. 3.2.1b illustrates the average residual noise levels on the sections between SSEs by the diameter of the circles, for the cGPS sites used in this study, superimposed on GoogleEarth imagery. 


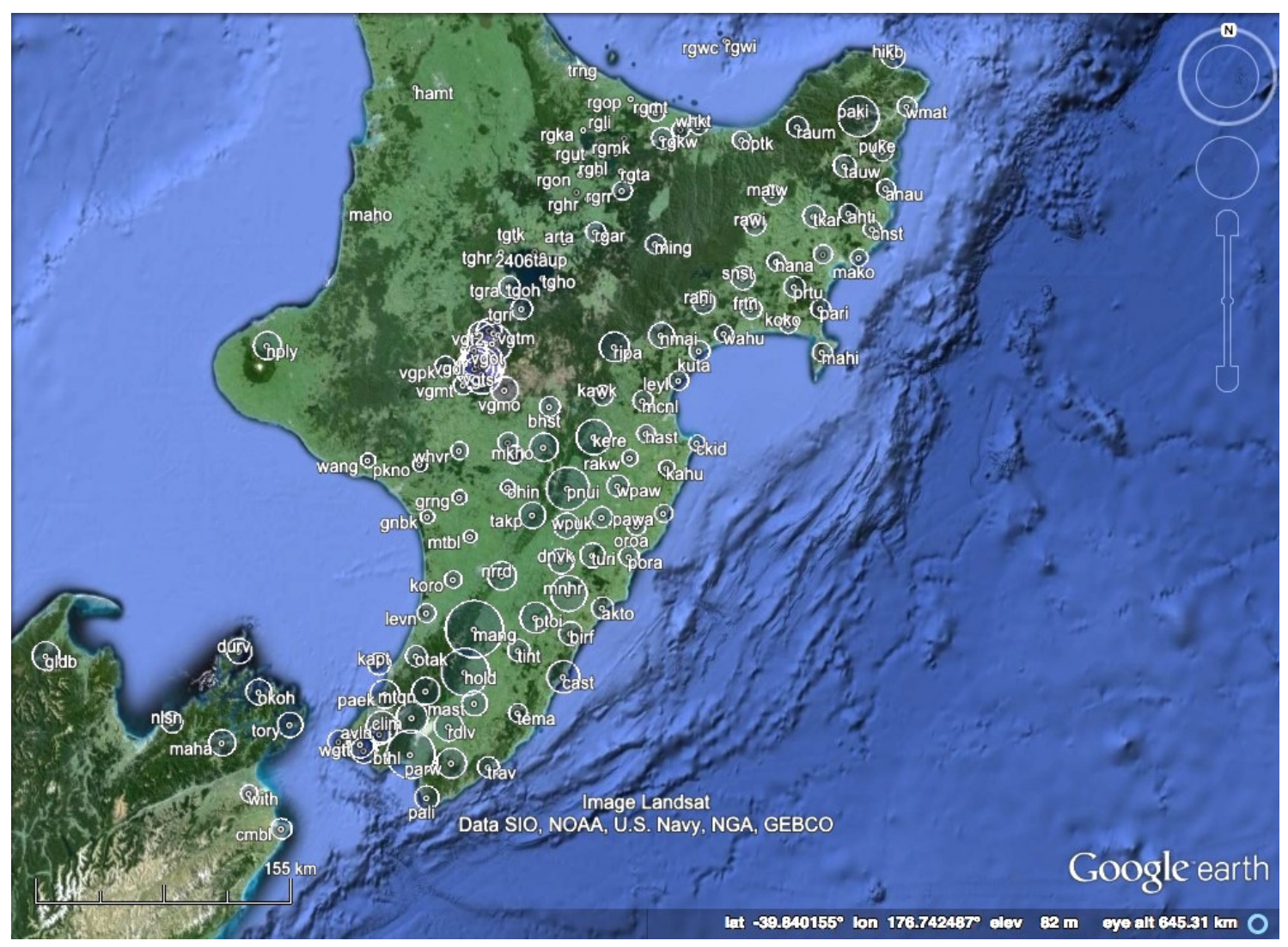

Figure 3.2.1b: Comparative rms average residual cGPS site noise levels, indicated by circle diameters, superimposed on GoogleEarth imagery. The largest circle, at MANG, represents an rms average of 6.7 $\mathrm{mm}$.

\subsection{Surface displacement offsets}

\subsubsection{Descriptive statistics}

The primary outcome of the processing of cGPS data is a list of dates and displacements at cGPS sites where it appears that an SSE or other discrete event has been detected (known as the 'answerout.xlsx' file). For the period from 01 Jan 2000 to 15 Feb 2014 this comprises 825 rows of data classified into 150 discrete 'events'. 151 rows (cGPS sites) (18\%) and 44 individual 'events' (29\%) showed displacements that appeared to move in a 'negative' direction (i.e. in a westerly direction on the east component). Displacements at individual sites on the E component varied from $-13 \mathrm{~mm}$ to $+56 \mathrm{~mm}$, and event durations at individual sites varied from 3 to 513 days. 
As indicated in section 2.4.5, each 'event' was categorized in terms of its 'quality', with Cat 1 being assigned to events showing clear shape development at more than 4 sites, and Cat 4 assigned to poor quality events of generally low amplitude and observed at a small numbers of sites. The events were also classified by the region in which they occurred, as indicated in Fig. 3.2.1a.

Figure 3.2.1a: Regions used for classifying event locations

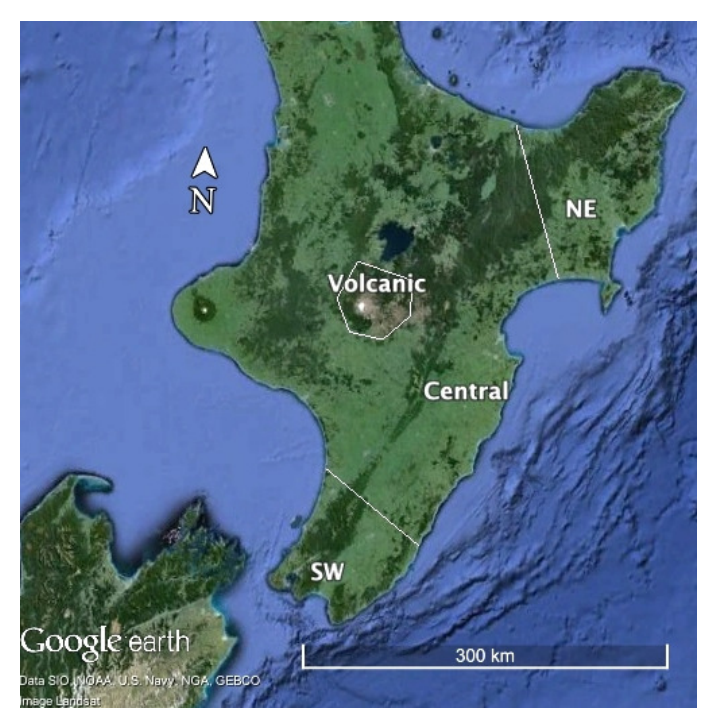

Surface displacement 'events', using E component data, are summarized in Table 3.2.1

\begin{tabular}{|c|c|c|c|c|c|c|}
\hline Region & Cat & $\begin{array}{c}\text { MeanDuration } \\
\text { days }\end{array}$ & Number & $\begin{array}{c}\text { meanD } \\
\text { Std.Dev. }\end{array}$ & $\begin{array}{c}\text { Mean MaxAmpl_E } \\
\text { mm }\end{array}$ & $\begin{array}{c}\text { MaxAmpl } \\
\text { Std.Dev. }\end{array}$ \\
\hline sw & 1 & 183.0 & 4 & 151.2 & 4.8 & 15.6 \\
\hline sw & 2 & 179.3 & 6 & 112.1 & 10.8 & 17.4 \\
\hline sw & 3 & 125.4 & 12 & 54.6 & 2.2 & 7.0 \\
\hline sw & 4 & 114.9 & 9 & 164.0 & $12.4^{*}$ & 25.1 \\
\hline ne & 1 & 67.5 & 12 & 69.6 & 10.8 & 16.0 \\
\hline ne & 2 & 35.2 & 14 & 19.3 & 10.3 & 8.0 \\
\hline ne & 3 & 41.5 & 35 & 31.6 & 3.4 & 5.4 \\
\hline ne & 4 & 37.8 & 18 & 30.9 & 1.4 & 3.0 \\
\hline c & 1 & 97.3 & 5 & 88.3 & 22.6 & 8.3 \\
\hline c & 2 & 77.5 & 9 & 78.8 & 5.6 & 6.3 \\
\hline c & 3 & 33.1 & 14 & 24.6 & 3.5 & 4.4 \\
\hline c & 4 & 22.9 & 5 & 16.1 & -3.0 & 6.5 \\
\hline v & 1 & & 0 & & & \\
\hline v & 2 & 77.4 & 3 & 22.5 & 1.1 & 9.2 \\
\hline v & 3 & 99.2 & 4 & 39.3 & -2.4 & 9.9 \\
\hline v & 4 & & 0 & & & 10.9 \\
\hline All Groups & & 67.8 & 150 & 76.1 & 5.5 & \\
\hline
\end{tabular}

Note: *Cat 4 in sw includes events connected with Cook Strait and Lake Grasmere earthquakes

Table 3.2.1: Breakdown of duration and maximum surface displacements in $E$ direction by region and "quality" of event (Cat $1=$ best, Cat 4 likely to be noise or disqualifying factors such as poor shape, small number of cGPS sites, or directly connected with earthquakes)

Categorization was based primarily on subjective assessment of event shape and closure of the error plots in 'SSE_cuts' (as in Fig 2.4.4b) and Appendix 9 examines whether 
categorization had any objective value. It suggests that there is some correlation between objective measures and the subjective categorization. Cat 1 'events' tended to best fit an exponential displacement-time model, of the 2 options used, whereas Cat 3 'events' tended to best fit a linear or 'ramp' model. Cat 2 contained approximately equal numbers of 'events' that fitted either a linear or exponential model.

The distributions of maximum surface displacements for Cat 1 to Cat 3 in the $\mathrm{E}$ direction, for positive and negative 'events' are shown in Fig. 3.2.1b. Fig. 3.2.1c shows the distribution of all median event durations for Cat 1 through Cat 3.

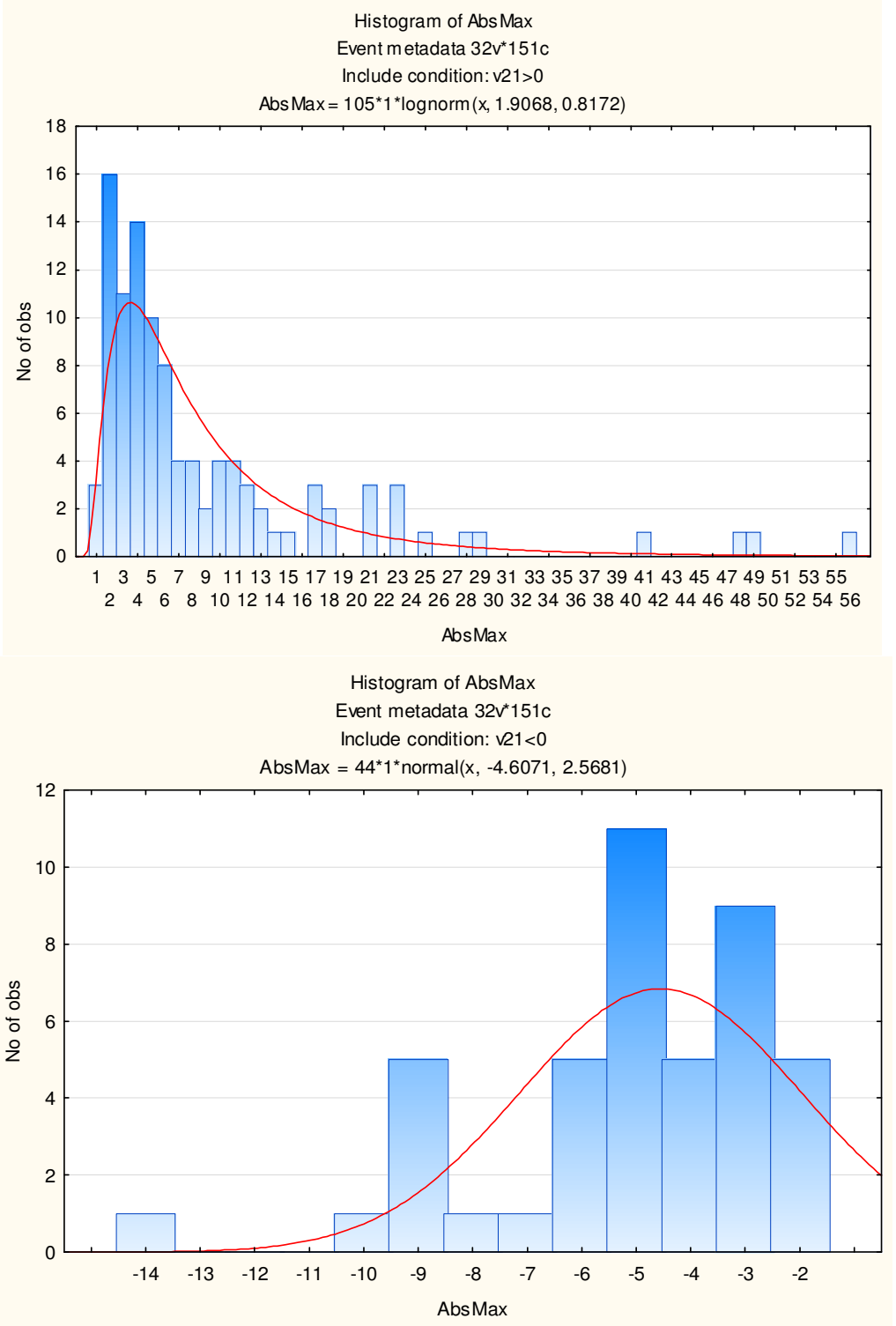

Figure 3.2.1b: Distribution of maximum E component displacements (mm) for "positive" events (with superimposed lognormal distribution), and "negative" events (with superimposed normal distribution), respectively. 


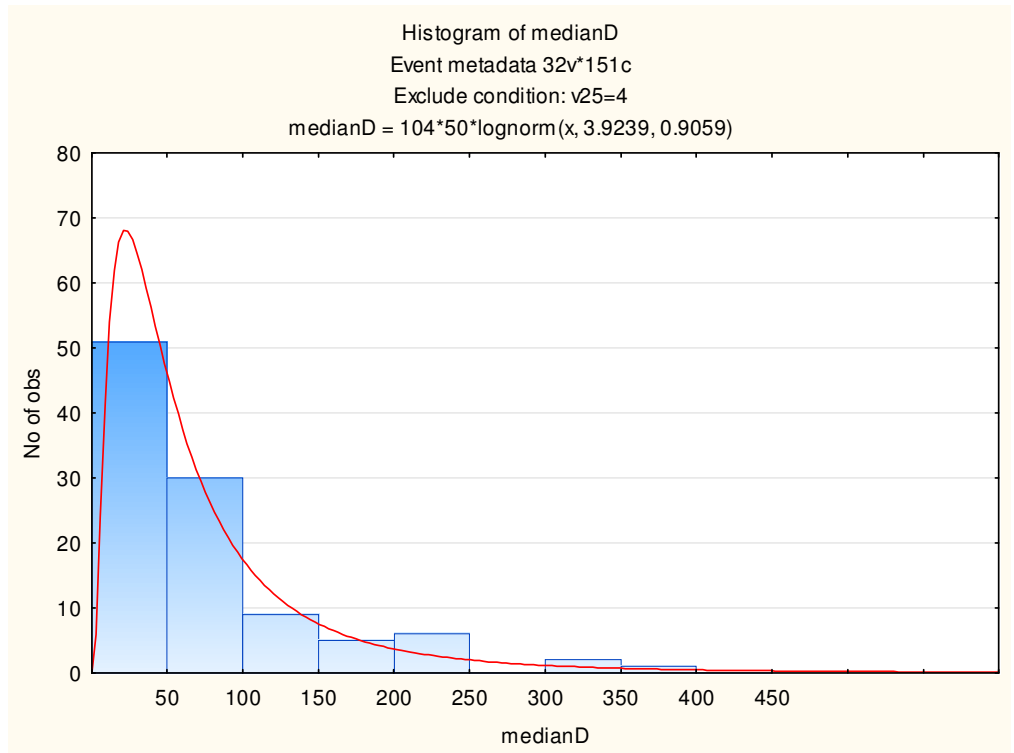

Figure 3.2.1c: Distribution of median event durations (days) for all Cat 1 through Cat 3 events, with superimposed lognormal distribution.

\subsubsection{Relationships between variables}

Fig. 3.2.2 illustrates a moderate degree of association between maximum E component displacements and mean 'event' durations for Cat 1 through Cat 3.

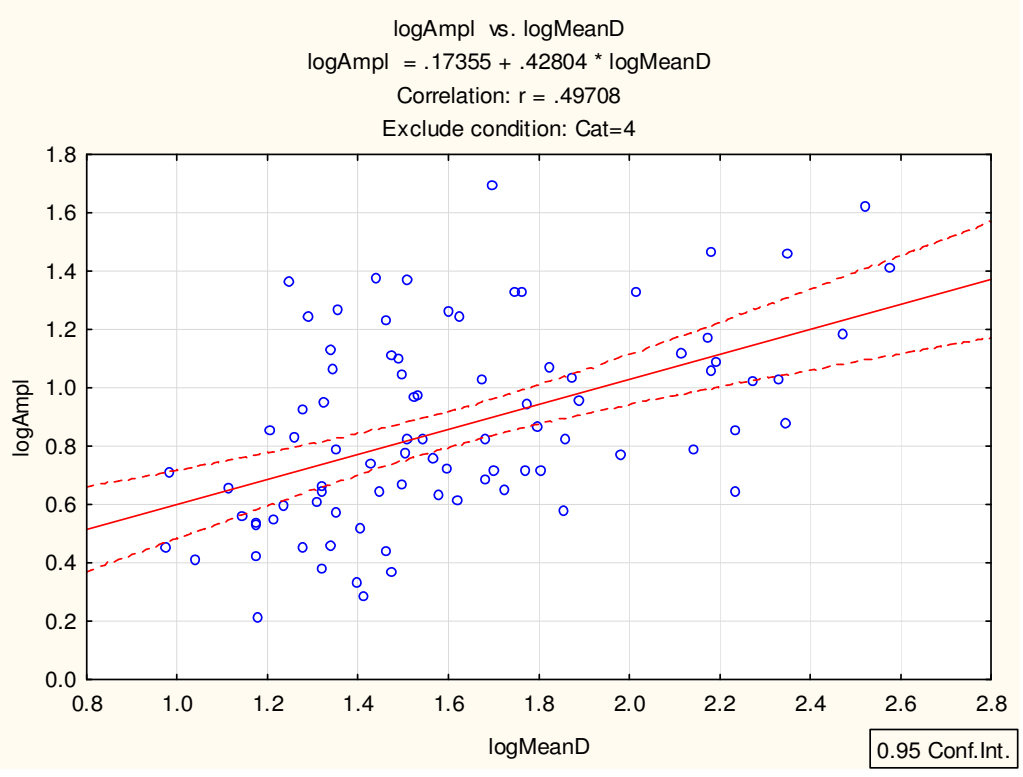

Figure 3.2.2: $\log$ (maximum E displacement amplitude) plotted against log (mean 'event' duration) for all Cat 1 through Cat 3 'events'. 
As is shown in Appendix 9, no correlation could be found between the time constant 'c' in the exponential model (see 2.4.4) and either amplitude or duration, or the variability of either. This is touched on further in section 5.3.

\subsubsection{Event displacement rate}

A new variable, the surface event displacement rate, was calculated simply as the amplitude of displacement at the cGPS site in mm divided by the duration of the 'event' in days. This is an approximation to the slope of the ramp in a linear model, in that it ignores any start and end processes. Fig. 3.2.3a shows the distribution of E component displacement rates (multiplied by 100) at individual sites. There is a strong tendency for approximately $75 \%$ of $E$ displacement rates to fall in the range 0.02 to $0.1 \mathrm{~mm} / \mathrm{day}$.

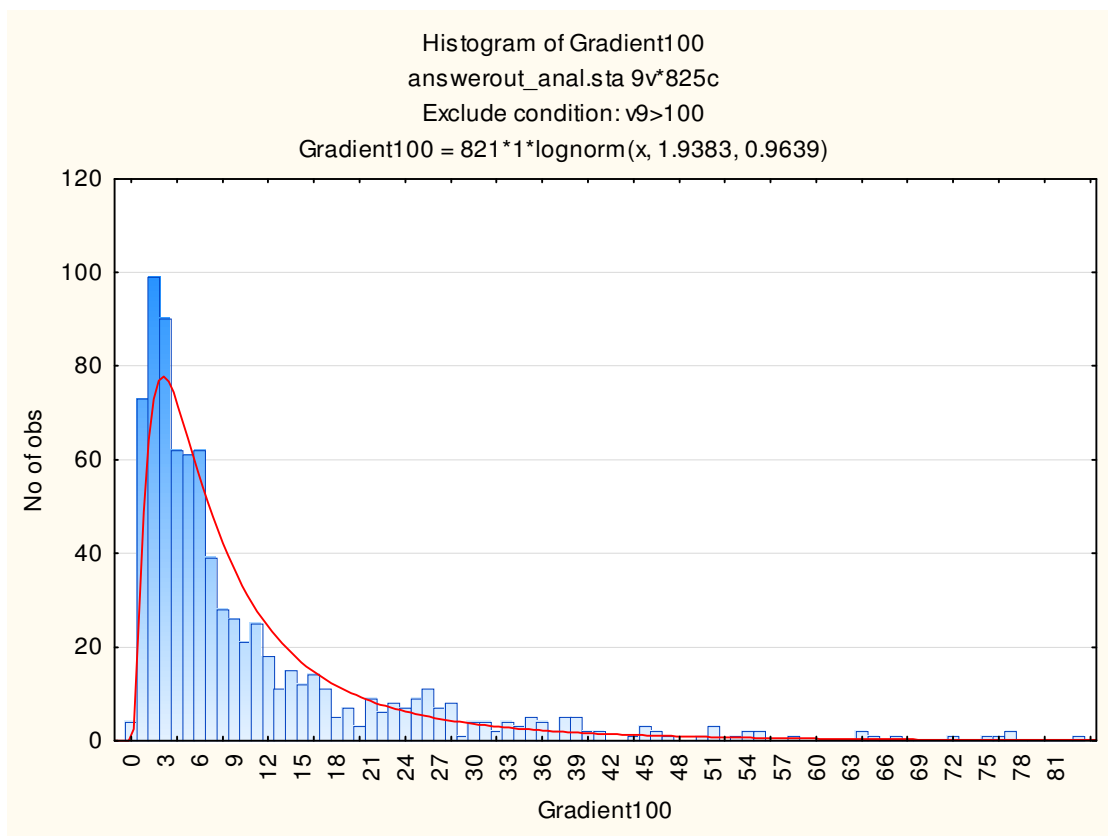

Figure 3.2.3a: Distribution of surface displacement rates in the easterly direction during SSE events together with superimposed lognormal distribution. The rates (in $\mathrm{mm} /$ day) have been multiplied by 100 for ease of display. Outliers (rate $>1 \mathrm{~mm} /$ day) have been removed.

If Cat 4 events are excluded (because they may, in some cases, simply be noise), the average displacement rates for positive 'events' are similar across all regions, except Cat 1 and Cat 2 events in the northeast, which appear to have event displacement velocities almost twice as those in the other regions - see Fig. 3.2.3b. The average 
displacement rates for the negative 'events' appear to be the same in all cases in all regions. Source slip rates are discussed in 5.4.2.5.
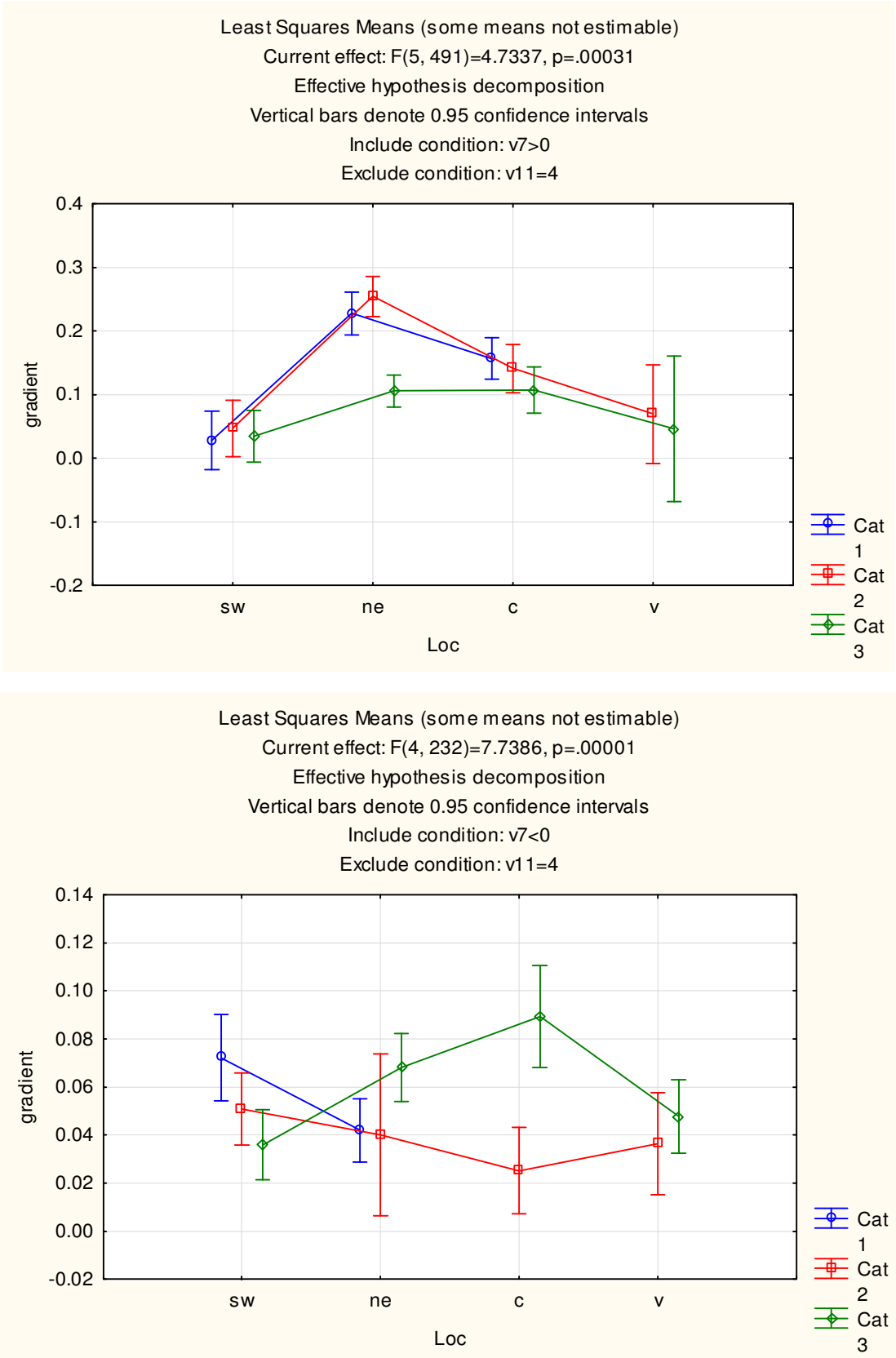

Figure 3.2.3b: Displacement rates ("gradient") in mm/day for Cat 1 through Cat 3 'events' grouped by region, for all positive (upper) and negative (lower) 'events'. Note different ordinate scales. Vertical bars indicate $95 \%$ confidence intervals.

It was observed that, particularly in the two regions with the highest number of 'events', there was a tendency for displacement rate to increase with amplitude of displacement. 
This tendency was greater in the northeast region - see Fig 3.2.3c. This was not apparent for negative 'events'.

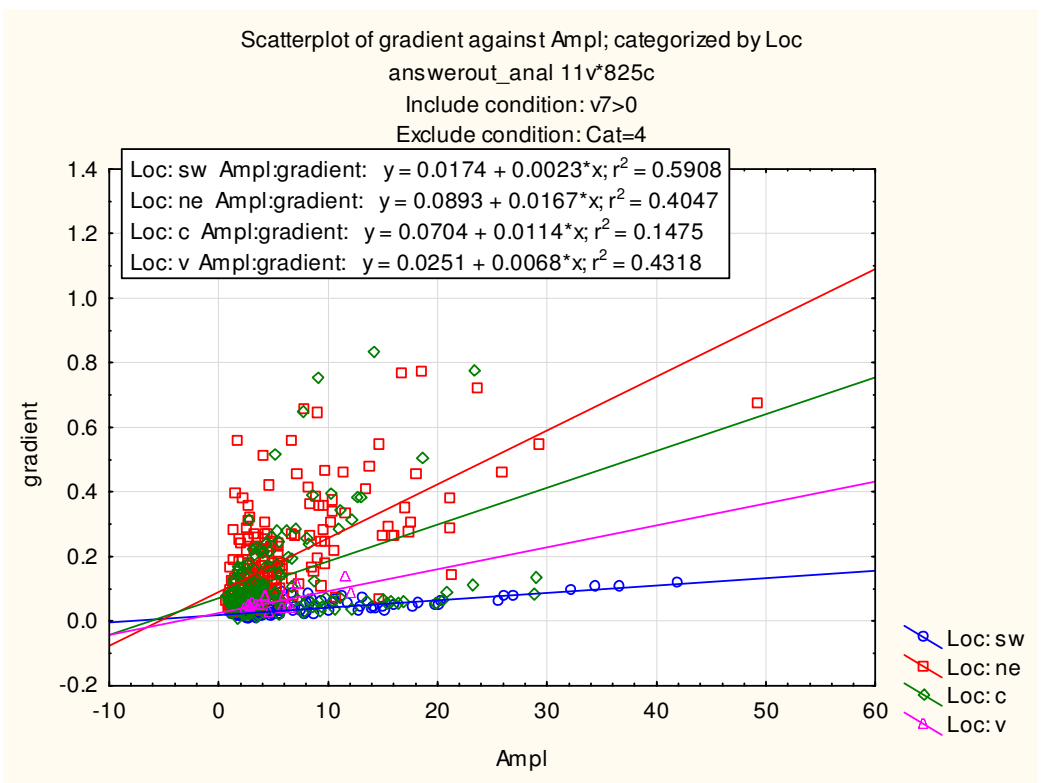

Figure 3.2.3c: Event displacement rate ('gradient' in $\mathrm{mm} / \mathrm{day}$ ) as a function of total event displacement ('Ampl', in the easterly direction) for all positive events, categorized by region.

\subsubsection{Scaling}

For the Cat 1 through Cat 3 surface displacement events, there was indication of a power law scaling effect, as shown in Fig 3.2.4a and b for displacement amplitude and duration respectively. Source event scaling is discussed in detail in section 5.4.2. 


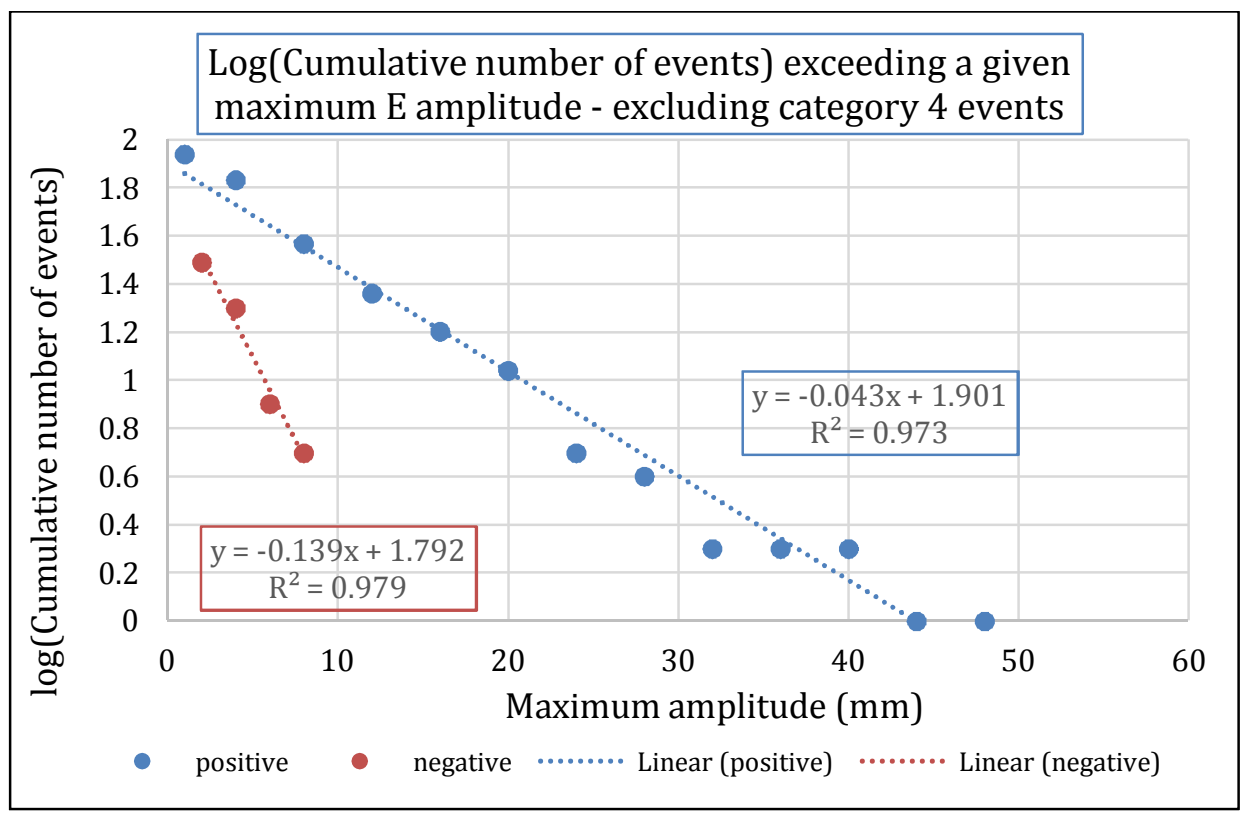

Figure 3.2.4a: $\log$ (cumulative number of events) versus maximum surface amplitude (E component) for both negative and positive displacements

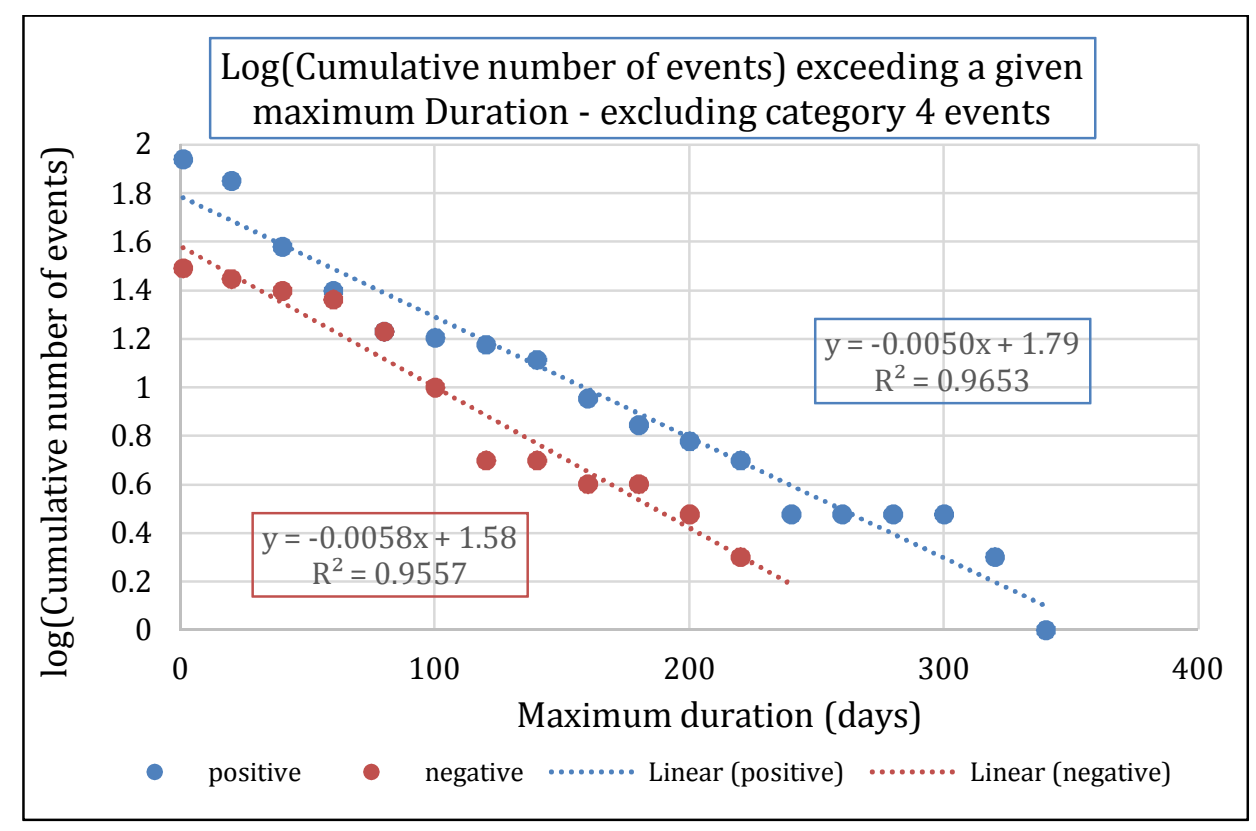

Figure 3.2.4b: $\log$ (cumulative number of events) versus maximum duration (E component) for both negative and positive displacements 


\subsubsection{Temporal evolution: shape of surface displacement-time curves}

As indicated in section 2.5, 'SPSS Tablecurve $2 D$ ' was used to assess the fit of stacked normalized displacements for Cat 1 'events' (i.e. those with the "best" or well-defined shapes) against 10 asymmetric transition functions:

Logistic Dose Response (LDR)

Log Normal Cumulative

Extreme Value Cumulative

Pulse Cumulative

Pulse Cumulative with Power Term

Weibull Cumulative

Asymmetric Sigmoid

Asymmetric Sigmoid Reverse Asymmetry

Cascade Formation

Impulse Transition

Cumulative EMG (Exponentially Modified Gaussian)

For each of these 'events' the asymmetric sigmoid reverse regression (AsymSigR) appeared to be the optimum from these models. An example of the fit for 'event' 86 is shown in Fig. 3.2.5a.

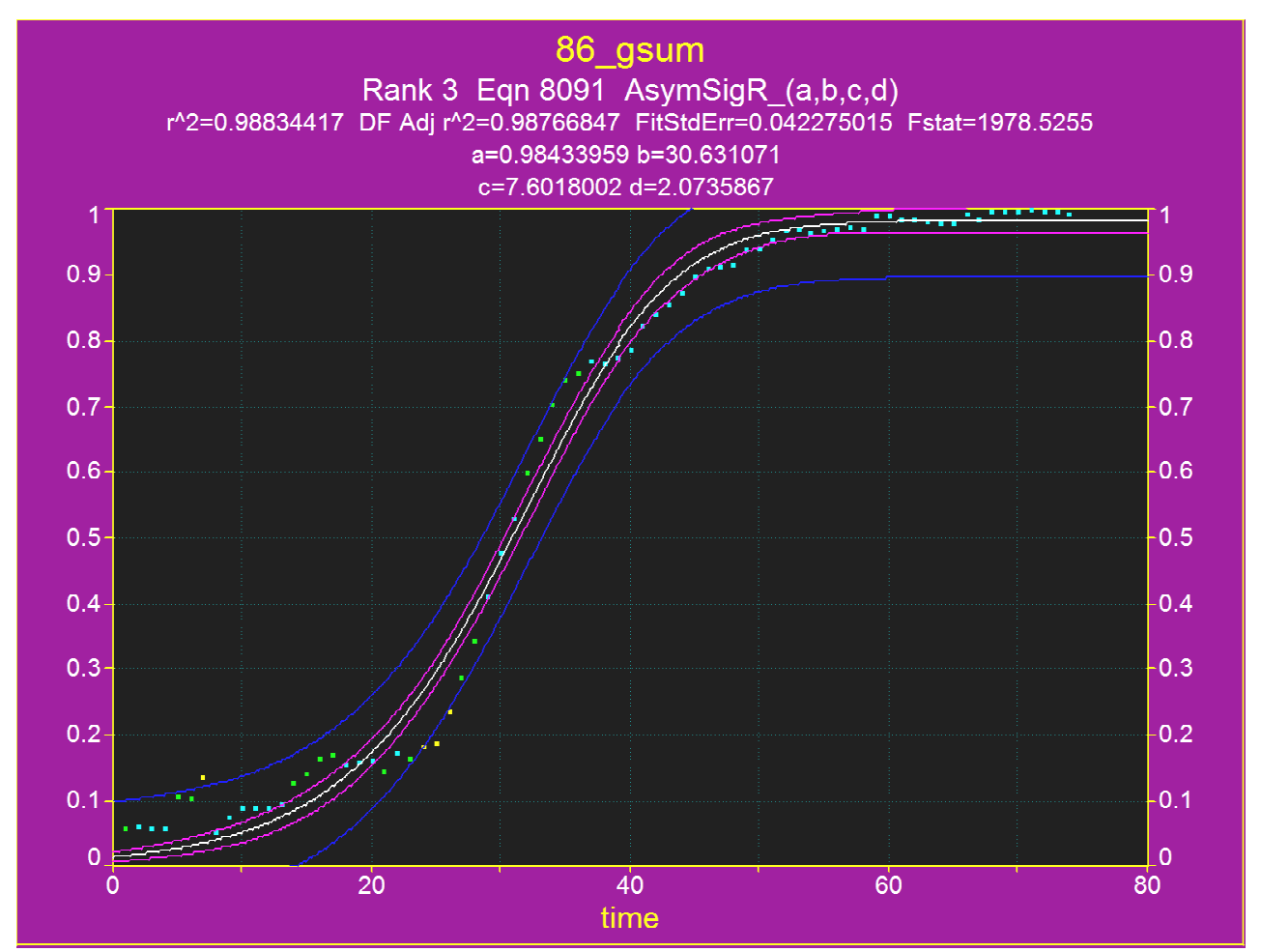

Figure 3.2.5a: Fit of reverse asymmetric sigmoid curve to 'event' 86 normalised stacked displacementtime data, using SPSS Tablecurve $2 D$. Dots indicate data points, the white line indicates the least squares best fit of the function, the magenta lines indicate the $95 \%$ confidence intervals on the best fit, and the blue lines indicate the $95 \%$ confidence intervals on predicted values. 
The non-intercept AsymSigR regression model involves 4 parameters:

$$
y=a\left[1-\left\{1+\exp \left(\frac{x+c \cdot \ln \left(2^{\frac{1}{d}-1}\right)-b}{c}\right)\right\}^{-d}\right]
$$

where:

$\mathrm{a}=$ maximum amplitude

$\mathrm{b}=$ abscissa distance from record start to mid-height intercept (inflexion point)

$\mathrm{c}=$ measure of abscissa width of the transform (i.e. between $\mathrm{Ymin}+25 \%$ (Ymax-Ymin) and Ymin $+75 \%$ (Ymax-Ymin))

$\mathrm{d}=$ non-linear measure of degree of asymmetry - small values extend the upper part of the sigmoid along the abscissa and large values extend the lower part

It is therefore expected that there will be some correlation between parameters ' $b$ ' and 'c', since longer events will be expected to have longer transition periods. However, it should be noted that ' $b$ ' includes both the half-width of the sigmoid plus any delay in the start of the curve, and therefore:

- 'events' of low amplitude, where detection of the start and finish times is more difficult and uncertain, and

- 'events' where the individual sites appear to show distinct differences between start times (perhaps suggesting movement of the source)

will both lead to increase of the ' $b$ ' parameter. For the stacked data, there was no significant correlation between parameters ' $b$ ' and ' $c$ '. However, for the 21 individual largest events, the Pearson correlation was 0.45 between these 2 parameters, which is significant at the 0.02 level.

It is noted that the stack can be 'contaminated' by the amplified noise of the smaller magnitude site records, particularly at the extremes, and specifically in the lower 'leading' edge - more so if the start times are not well-aligned - which could lead to a perception of a leading edge where one may not exist.

Of the 21 events initially nominated as category 1, (comprising 16 positive and 5 negative events, 12 from NE, 6 from $\mathrm{C}$, and 3 from SW), 14 of 16 positives show stack 
$\mathrm{R}^{2}$ values exceeding $90 \%$, and 4 of the 5 negatives show $\mathrm{R}^{2}$ values above $80 \%$ (where $\mathrm{R}^{2}$ indicated the percentage of total variance explained by the AsymSigR regression).

Additionally, for each of these events, in view of the noted caution concerning the possibly misleading leading edge of a stacked shape, the largest amplitude individual site record was also examined against this regression model. All positive events showed $\mathrm{R}^{2}$ values in excess of $87 \%$, with most above $97 \%$ for the AsymSigR model. The negative events showed a more mixed bag, with only 2 above $95 \%$.

The regression results for both the stacked normalized data and the single largest site displacement data are shown in Table 3.2.5.

\begin{tabular}{|c|c|c|c|c|c|c|c|c|c|c|c|c|c|}
\hline \multirow[b]{2}{*}{ Region } & \multirow[b]{2}{*}{ event } & \multicolumn{6}{|c|}{ Stack } & \multicolumn{6}{|c|}{ Largest individual record } \\
\hline & & a & b & c & d & $\mathbf{R}^{\wedge} \mathbf{2}$ & seFit & a & b & c & d & $R^{\wedge} \mathbf{2}$ & seFit \\
\hline C & 16 & 1.246 & 106 & 27.9 & 0.26 & 0.982 & 0.040 & 28 & 113 & 178.7 & 2.98 & 0.995 & 0.67 \\
\hline c & 29 & 0.896 & 21 & 10.7 & $3.80 \mathrm{E}+12$ & 0.922 & 0.088 & 23 & 22 & 1.163 & 0.234 & 0.997 & 0.57 \\
\hline SW & 41 & 1.033 & 182 & 32.2 & 0.13 & 0.962 & 0.031 & 14 & 109 & 46.4 & $4.90 E+15$ & 0.974 & 0.77 \\
\hline C- & 48 & 0.727 & 16 & 2.28 & 0.134 & 0.834 & 0.077 & 16 & 210 & 14.4 & 0.051 & 0.750 & 1.10 \\
\hline SW & 65 & 0.851 & 32 & 25.2 & 6.48 & 0.911 & 0.074 & 3 & 28 & 0.033 & 0.0026 & 0.868 & 0.52 \\
\hline NE & 86 & 0.984 & 31 & 7.6 & 2.07 & 0.988 & 0.042 & 46 & 31 & 2.18 & 0.288 & 0.998 & 0.94 \\
\hline $\mathrm{NE}$ & 89 & 1.009 & 16 & 10.7 & 0.942 & 0.924 & 0.074 & 5 & 8 & 0.121 & 0.0086 & 0.913 & 0.34 \\
\hline $\mathrm{C}$ & 97 & 0.797 & 84 & 20.2 & 0.414 & 0.975 & 0.042 & 22 & 84 & 13.6 & 0.339 & 0.980 & 1.17 \\
\hline NE - & 98 & 0.656 & 25 & 0.222 & 0.0055 & 0.835 & 0.065 & 9 & 36 & 1.74 & 0.0398 & 0.984 & 0.18 \\
\hline C & 99 & 1.007 & 21 & 0.482 & 0.0176 & 0.953 & 0.060 & 9 & 21 & 0.0425 & 0.0015 & 0.988 & 0.25 \\
\hline NE & 101 & 1.025 & 16 & 12.7 & $1.29 \mathrm{E}+14$ & 0.876 & 0.101 & 4 & 16 & 0.0363 & 0.0018 & 0.979 & 0.14 \\
\hline NE & 104 & 0.933 & 13 & 3.14 & $1.90 \mathrm{E}+17$ & 0.982 & 0.055 & 8 & 13 & 4.08 & $2.80 \mathrm{E}+17$ & 0.995 & 0.21 \\
\hline NE & 117 & 0.947 & 17 & 4.53 & 1.504 & 0.991 & 0.033 & 16 & 13 & 2.006 & 0.304 & 0.998 & 0.22 \\
\hline NE & 118 & 0.926 & 9 & 1.78 & 0.955 & 0.988 & 0.041 & 10 & 8 & 1.735 & 0.966 & 0.997 & 0.23 \\
\hline$N E$ & 132 & 0.979 & 12 & 2.48 & 0.447 & 0.997 & 0.021 & 12 & 15 & 2.082 & 0.603 & 0.999 & 0.10 \\
\hline NE - & 133 & 0.905 & 40 & 0.0985 & 0.0017 & 0.924 & 0.064 & 20 & 177 & 31 & 0.162 & 0.752 & 1.21 \\
\hline $\mathrm{C}$ & 134 & 0.961 & 11 & 2.168 & 1.557 & 0.997 & 0.020 & 23 & 11 & 1.708 & 0.834 & 0.998 & 0.50 \\
\hline NE - & 136 & 0.505 & 40 & 57.6 & $2.70 \mathrm{E}+10$ & 0.656 & 0.091 & 2 & 62 & 56.5 & $9.20 \mathrm{E}+17$ & 0.528 & 0.51 \\
\hline $\mathrm{NE}$ & 139 & 0.729 & 15 & 4.83 & 0.542 & 0.763 & 0.114 & 18 & 18 & 2.088 & 0.212 & 0.999 & 0.21 \\
\hline SW - & 140 & 0.919 & 16 & 4.36 & 0.401 & 0.929 & 0.082 & 8 & 17 & 1.007 & 0.086 & 0.953 & 0.65 \\
\hline \multirow[t]{9}{*}{$\mathrm{NE}$} & 146 & 0.968 & 12 & 2.02 & 0.732 & 0.999 & 0.016 & 18 & 12 & 1.63 & 0.396 & 0.999 & 0.12 \\
\hline & \multirow[t]{8}{*}{ Notes: } & & \multicolumn{11}{|c|}{ least reliable } \\
\hline & & $x x-$ & \multicolumn{11}{|c|}{ negative amplitude } \\
\hline & & a & \multicolumn{11}{|c|}{ for Stack, should be close to 1 (normalised amplitude). For individual = max amplitude } \\
\hline & & $\mathrm{b}$ & \multicolumn{11}{|c|}{ zero to inflexion point $=$ half duration in days } \\
\hline & & c & \multicolumn{11}{|c|}{ measure of transition width } \\
\hline & & $d$ & \multicolumn{11}{|c|}{ measure of asymmetry } \\
\hline & & $\mathrm{R}^{\wedge} 2$ & \multicolumn{11}{|c|}{ proportion of total variance explained by the regression } \\
\hline & & seFit & \multicolumn{11}{|c|}{ standard error of fit, for Stack, multiply by $100=\%$ of maximum amplitude, else $\mathrm{mm}$ amplitude } \\
\hline
\end{tabular}

Table 3.2.5: Regression results for Cat 1 'events' against Reverse Asymmetric Sigmoid function, for both the stacked normalized data, and the largest individual site record for the corresponding event.

Additionally, 8 Cat 1 events were examined in detail - comprising 6 positive (2 each from NE, C and SW) and 2 negative (1 each from NE and C) - in total 88 individual site 
records ranging in duration from 20 to over 200 days and event amplitudes from 1.3 to $46 \mathrm{~mm}$.

\section{Positive events}

For the 61 positive individual site records, 5 gave no fits to the transition models. The remaining 56 records showed $\mathrm{R}^{2}$ values for the AsymSigR model ranging from $37 \%$ to $99.9 \%$ with an average of $90 \%$. 14 of the 56 records were recorded as "bad fits", mainly because of an ill-defined (or lacking) upper asymptote (such that the 'a' values were in excess of the observed maximum amplitude) - nevertheless many of these fits had a high $\mathrm{R}^{2}$ value. At this stage it should be noted that whilst this may suggest that the AsymSigR model is thus not universally useful, in some senses this outcome is simply a combined artifact of:

- the 'Tablecurve 2D' algorithm, which seeks the best fit to the data, without any preconstraints, and

- Inconsistent and sometimes over-tight constraints placed on the picking of the endtimes in 'SSE_cuts'.

If the ' $a$ ' value were to be pre-constrained as being within $+/$ - some tolerance of the maximum amplitude, this problem might be overcome.

An alternative algorithmic route to determining the success or otherwise of the AsymSigR regression was sought, using the parameter values, coefficient of determination and residual standard error values with discrimination statistics. This was considered unsuccessful in that whilst regression acceptability could be determined $100 \%$ of the time, failure was only predicted $70 \%$ of the time - in other words, approximately $1 / 3$ of unacceptable 'shapes' would be selected as acceptable by this method. It would thus seem more practical to either re-pick 'event' end-times to ensure adequate definition of the 'death' part of the curve, or to pre-constrain the values of parameter ' $a$ '.

With respect to the correlation between ' $b$ ' and 'c' parameter values, using the larger set of individual site records for positive events, and including even those regressions judged unacceptable, the Pearson correlation coefficient was 0.58 , significant at the 0.0001 level. As may be seen in Fig. 3.2.5b, the satisfactory/unsatisfactory categorization played no significant part in this correlation. 


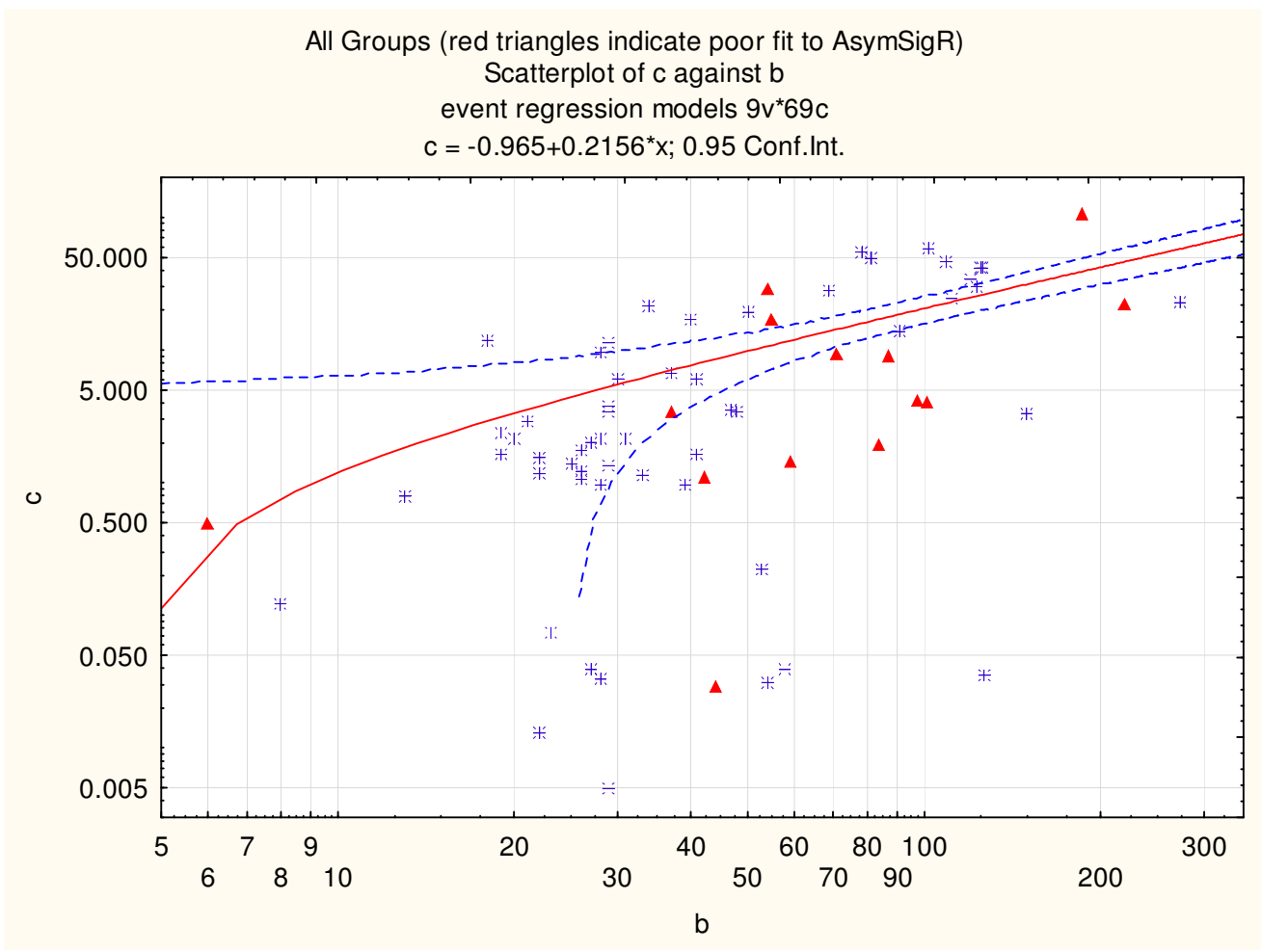

Figure 3.2.5b: Correlation between parameters ' $b$ ' and ' $c$ ' on individual site records for AsymSigR regression. Red triangles indicate results where the regression fit was considered unsatisfactory.

The stacked versions of 18 Cat 2 events were also examined against the AsymSigR model. One very noisy 'event' (E1) had a very poor fit to any transition model, but the remainder fitted satisfactorily, with $\mathrm{R}^{2}$ values ranging from $71 \%$ to $99 \%$, with an average of $92 \%$. As with the category 1 events, some did not asymptote to 1.0 , but again, this could be overcome by constraining parameter 'a', or, preferably, by extending the data length.

Negative events

It became clear that the AsymSigR curve was not the best fit for the individual negative events, and it was possible that the apparently reasonable fit for the stacked results could well have been the consequence of leading-edge contamination. Most of the individual negative event records started abruptly rather than having any significant leading edge. It was therefore decided to select a simple logarithmic regression model that provided a reasonable fit in most cases:

$$
\mathrm{Y}=\mathrm{a}+\mathrm{b} \ln (\mathrm{x})
$$


Whilst this was not usually the first model chosen by Tablecurve $2 D$, it should be recognized that most negative events are of low magnitude and the signal to noise ratio is relatively poor. The individual $\mathrm{R}^{2}$ values ranged from $28 \%$ to $94 \%$, with an average of $69 \%$. A typical example of an individual negative record is shown in Fig. 3.2.5c.

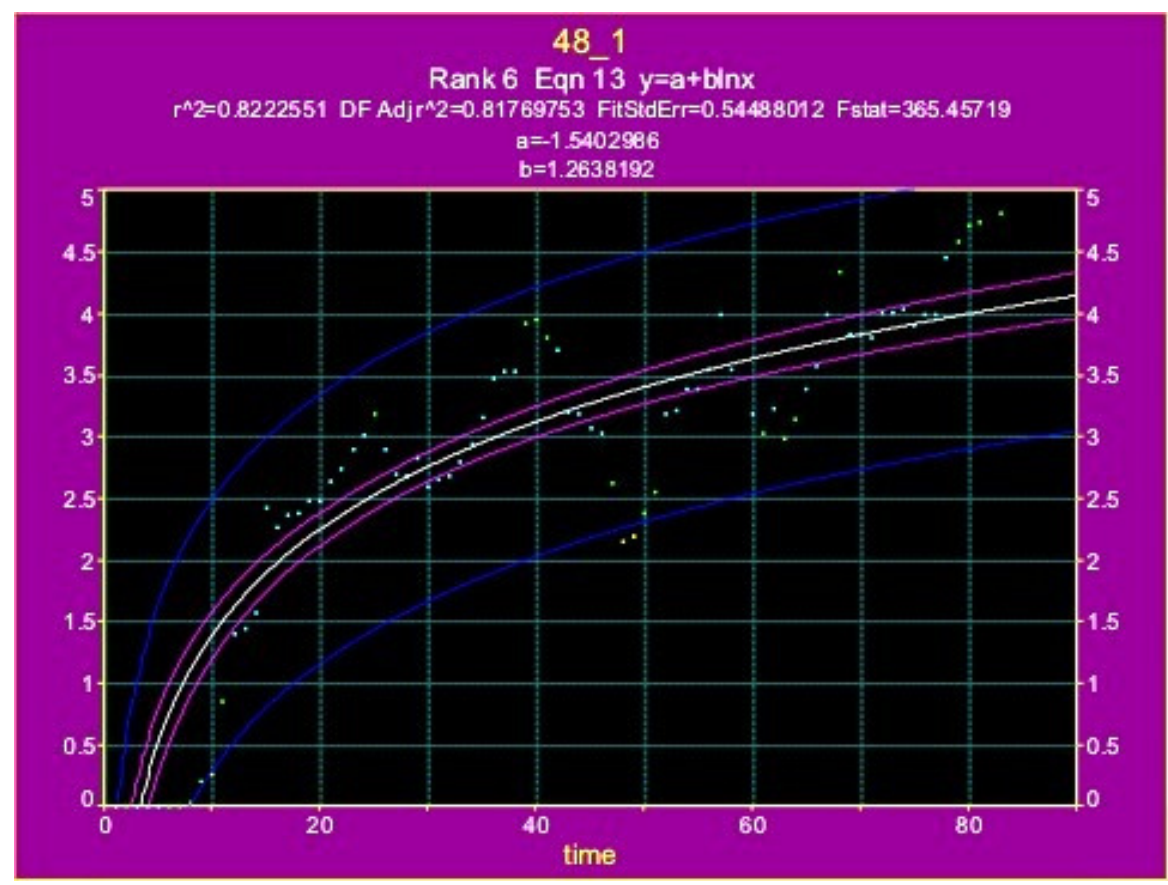

Figure 3.2.5c: Example of logarithmic fit to an individual record for negative event 48

An additional review was undertaken of all the stacks for negative events. In addition to the logarithmic fit suggested, note was also taken of fits to exponential and linear models. Of the 28 negative events, 18 showed better fit to a linear model rather than to the other models, and 6 events showed a better fit to the logarithmic model. In no case was an exponential model significantly better than the other two. The 6 events showing a better fit to the logarithmic model were $33(\mathrm{~V}), 55(\mathrm{~V}), 82(\mathrm{NE}), 83(\mathrm{~V}), 98(\mathrm{NE})$ and 129 (C). Appendix 10 shows an example of the ranked function fits for the stacked normalized 'event' 13. 


\subsection{Secular velocities}

Once the records for each site have been cleaned of periodic (seasonal) noise and outliers, an estimate can be made of the secular velocity at each site (averaged over the life of each site, to a maximum of 14 years). Two estimates are possible:

- an overall average, ignoring SSEs

- an average after removal of SSE displacements (i.e. 'SSE-free', which may be considered indicative of an underlying inter-SSE motion). This can be considered a benchmark from which SSEs can be differentiated.

Fig. 3.3a shows comparative average horizontal secular velocity vectors at each cGPS site, together with the average vectors due to SSEs.

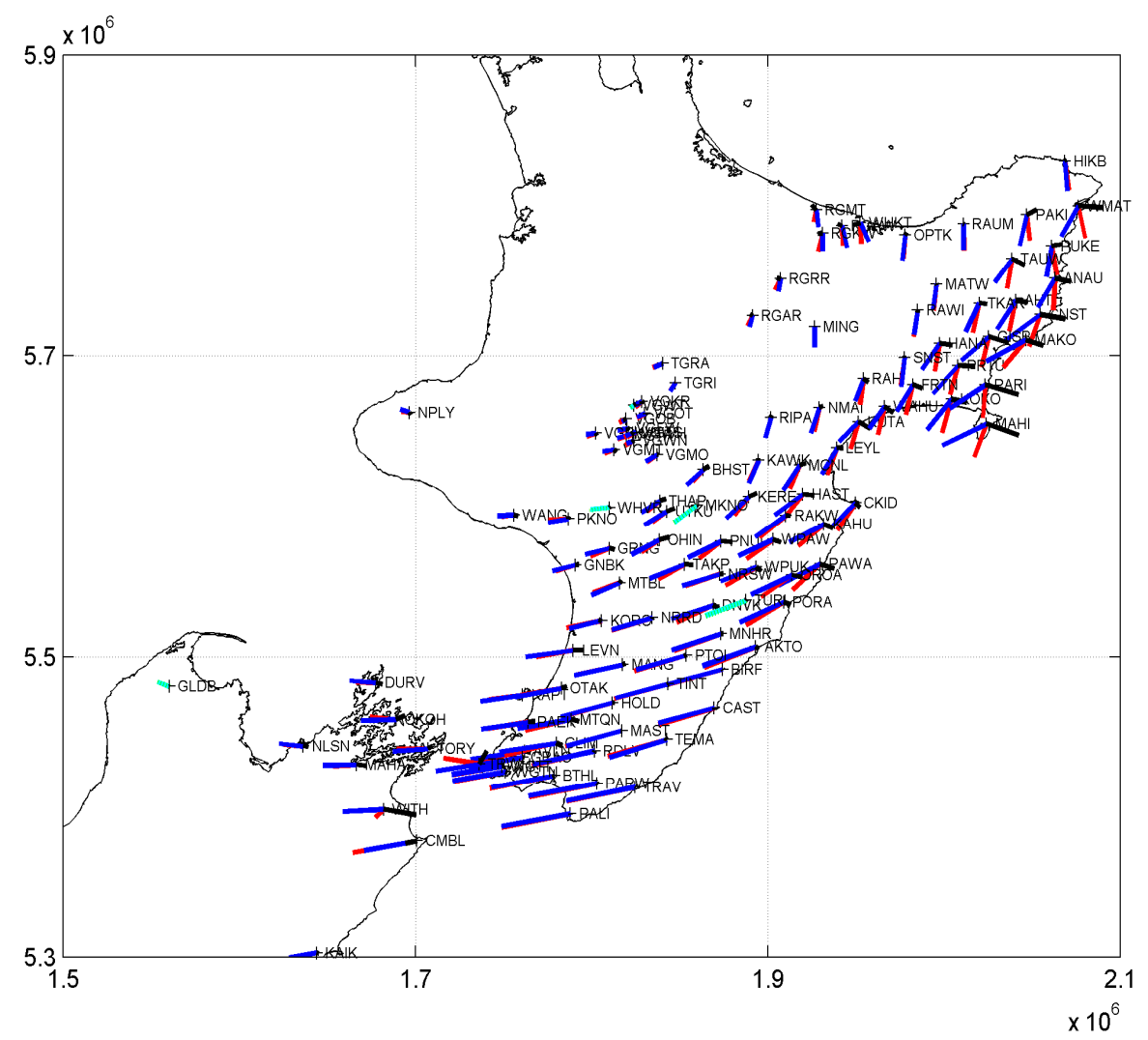

Figure 3.3a: Comparative average horizontal secular velocity vectors for the cGPS sites in this study referenced against AUCK. Blue lines indicate the 'SSE-free' magnitude and direction; black lines indicate the average velocity component due to SSEs; red lines indicate the overall average including SSEs; and cyan lines indicate sites for which no SSEs were detected (and therefore the two velocity estimates are the same). Note that the SSE velocities were obtained as 'overall average' minus 'SSEfree'. Scaling: PALI (most southerly site on the North Island) has an SSE-free vector length equivalent to $40 \mathrm{~mm} / \mathrm{yr}$. 
Fig. 3.3b shows comparative average vertical secular velocity vectors, and since the values are relatively small outside the TVZ, only the overall averages are shown (i.e. including SSEs). In both cases the results have been referenced against AUCK.

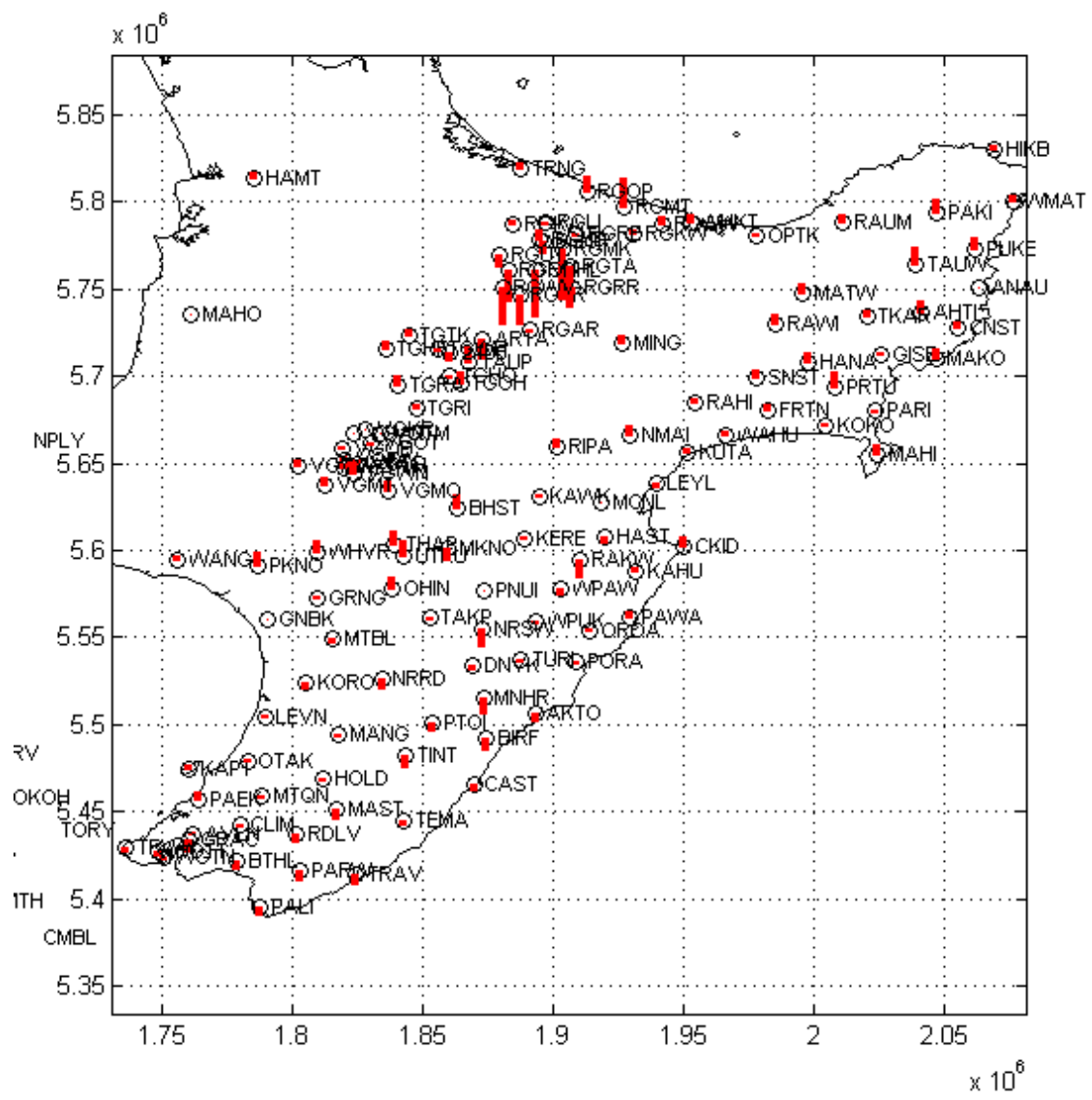

Figure 3.3.b: Comparative overall average vertical secular velocities (inclusive of SSEs) for North Island cGPS sites, referenced against AUCK. Lines pointing south indicate displacement downwards, and vice versa. Note that the vector lengths are exaggerated by a factor of 2 compared with the scale used in Fig. 3.3a. Scaling: TAUW on the Raukumara peninsula has a vector length equivalent to an upwards velocity of $5 \mathrm{~mm} / \mathrm{yr}$.

Appendix 11 tabulates the average secular velocity components for the cGPS sites in this study, both with and without SSEs. The table may be compared directly with Table 1 in Lamb and Smith [2013], although it should be noted that the current work covers a longer period and less sites. Lamb and Smith used the Australian Plate as their reference, whereas in this project, velocity components are referenced to AUCK. It will be observed that the overall secular velocities at CMBL and WITH include movement caused by the Cook Strait and Lake Grasmere earthquakes, and hence the very large rms velocity residuals values, particularly in the east component. 
The stability of the site AUCK, compared with the Australian plate reference can be verified by comparing the ITRF 2000 velocities for two of the New Zealand cGPS sites as originally reported by Beavan et al. [2002], with velocities reported by Lamb and Smith [2013] (who suggested that the north western sites of the north island could be taken as a stable reference frame), and those obtained in this project - see table 3.3.

\begin{tabular}{|l|cccc|cccc|}
\hline ITRF 2000 & \multicolumn{4}{|c|}{ AUCK } & \multicolumn{4}{c|}{ WGTN } \\
& Vn & Ve & sigma vn & sigma ve & Vn & Ve & sigma vn & sigma ve \\
\hline Beavan et al. 2002 & 38.6 & 3.9 & $0.5^{\mathrm{a}}$ & 0.5 & 31.4 & -24.4 & 0.7 & 0.8 \\
Lamb \& Smith 2013 & 37.7 & 2.2 & $0.04^{\mathrm{b}}$ & 0.06 & 31.7 & -27.0 & 0.04 & 0.06 \\
This report & 39.4 & 4.6 & $2.0^{\mathrm{c}}$ & 3.4 & 33.1 & -24.4 & 2.9 & 4.0 \\
\hline
\end{tabular}

Note: uncertainties reported by (a) Beavan et al. as 1 sigma estimates by combining a white noise and a random walk noise model, (b) Lamb \& Smith as root mean square misfit on linear regression of deseasoned data over $>5 y$ rs, and in this report (c) as root mean square error to a linear regression on the full time series after noise reduction.

Table 3.3: Comparison of ITRF 2000 horizontal velocities at 2 North Island sites.

It can be seen that despite the differences in the expressions of uncertainty, the horizontal secular velocities reported in all three cases appear within the bounds of error - or at least sufficiently close as to not significantly affect the following analyses.
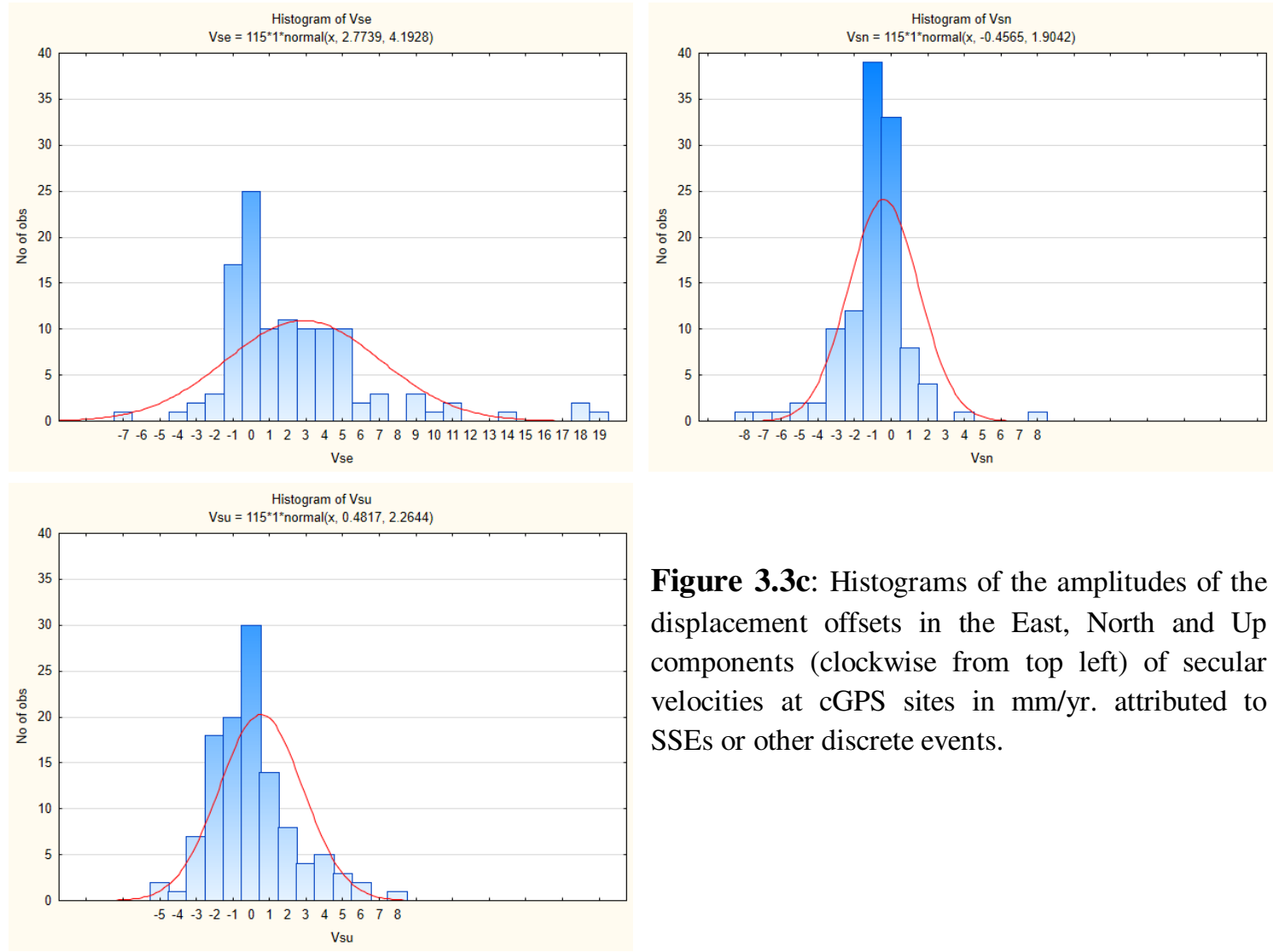

Figure 3.3c: Histograms of the amplitudes of the displacement offsets in the East, North and Up components (clockwise from top left) of secular velocities at cGPS sites in $\mathrm{mm} / \mathrm{yr}$. attributed to SSEs or other discrete events. 
Histograms of the amplitudes of displacement offsets in the $\mathrm{E}, \mathrm{N}$ and $\mathrm{U}$ components of the average secular velocities at cGPS sites attributed to SSEs or other discrete events, are shown in Fig. 3.3c. Both $\mathrm{N}$ and $\mathrm{U}$ component means were centred close to zero, whereas E component averages were more widely distributed, with values up to 19 $\mathrm{mm} / \mathrm{yr}$.

For small regional areas, over which it appears that displacement fields are relatively uniform, we might consider the average displacement field $\mathbf{D}$ to comprise a tensor of small strains and small rotations. This tensor can then be decomposed into the symmetric (strain) and the asymmetric (rotation) components [Marjetič et al., 2010]:

$$
\mathbf{D}=\boldsymbol{\varepsilon}+\boldsymbol{\omega}
$$

where: $\boldsymbol{\varepsilon}$ represents the strain component, and $\boldsymbol{\omega}$ represents the rotation component. It should be noted that the rotation component does not necessarily reflect rigid body rotation on geological time scales, but may simply represent strain asymmetry.

Using Matlab code written by Prof. E. Smith, both the average SSE velocities and the SSE-free velocities have been decomposed into strain and rotation rates for 7 regions in the North Island. The results are shown in Appendix 12 and summarized in Figs. 3.3d and 3.3e. The regional boundaries were estimated subjectively based on minimizing residual errors. 


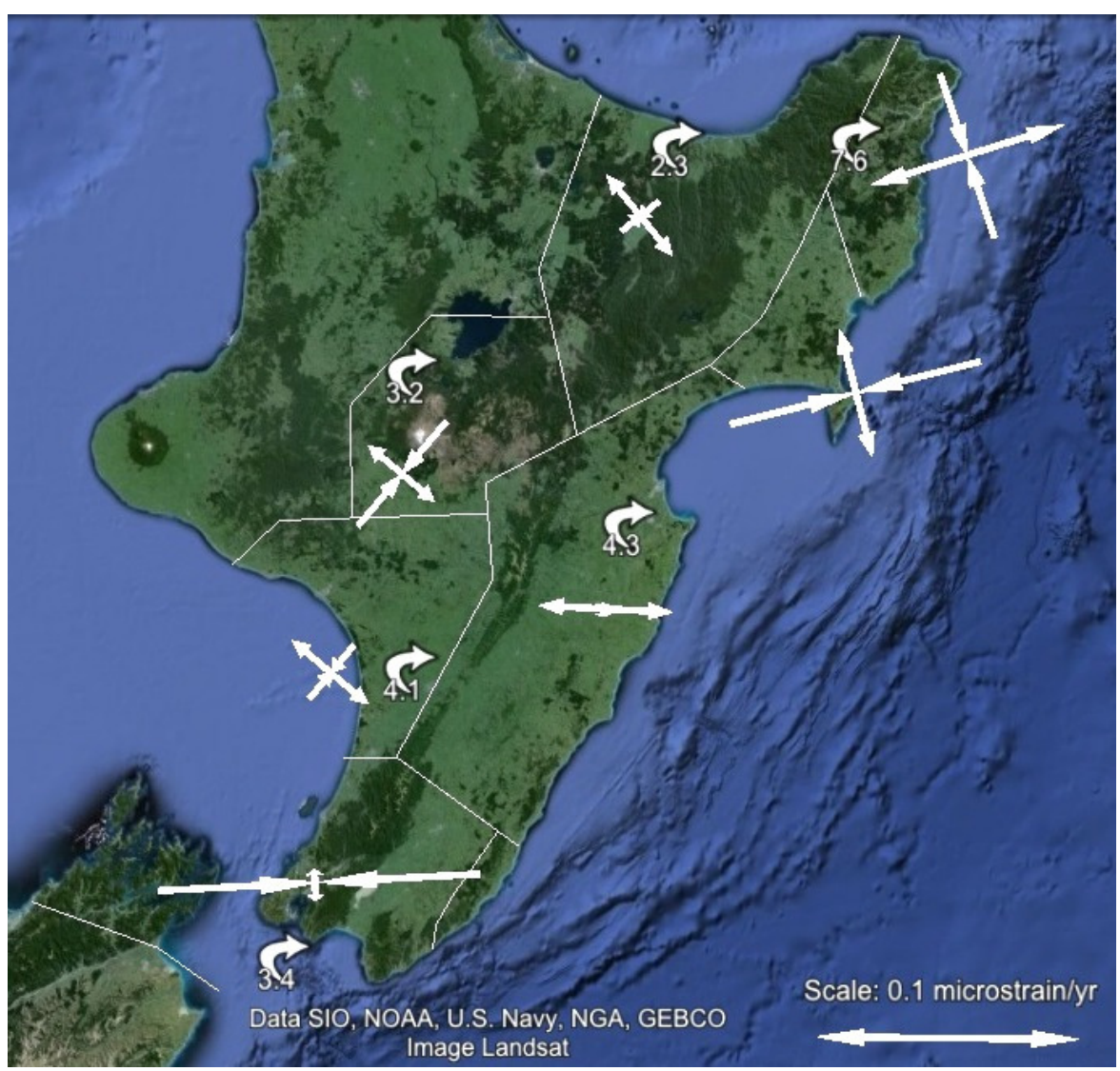

Figure 3.3d: Direction and magnitude representations of principal strain rates and rotation rates in 7 regions, derived from the SSE-free secular velocities. Rotation component rates are in degrees/Ma, with the rotation symbols located close to the poles calculated for each region. Background $\odot$ GoogleEarth.

Also shown in Appendix 12 is an analysis for the combination of the northeast coast and Mahia-Gisborne regions. This illustrates that the strain rate magnitudes and rotation rates are very sensitive to the number of sites selected. The results are also sensitive to the selection of the reference site within the group. However, in general terms, the azimuths of the principal strains appear relatively robust, and not significantly affected by these choices.

In Fig. 3.3e, for the average SSE (or other discrete event) displacement fields, no rotation poles are shown, since, with the exception of Ruapehu (for which the rotation component is small), these poles are located well outside the regions, and the rotation rates are generally small. In this case, the rotation components are almost certainly simply indications of a small degree of asymmetry in the strain field. 


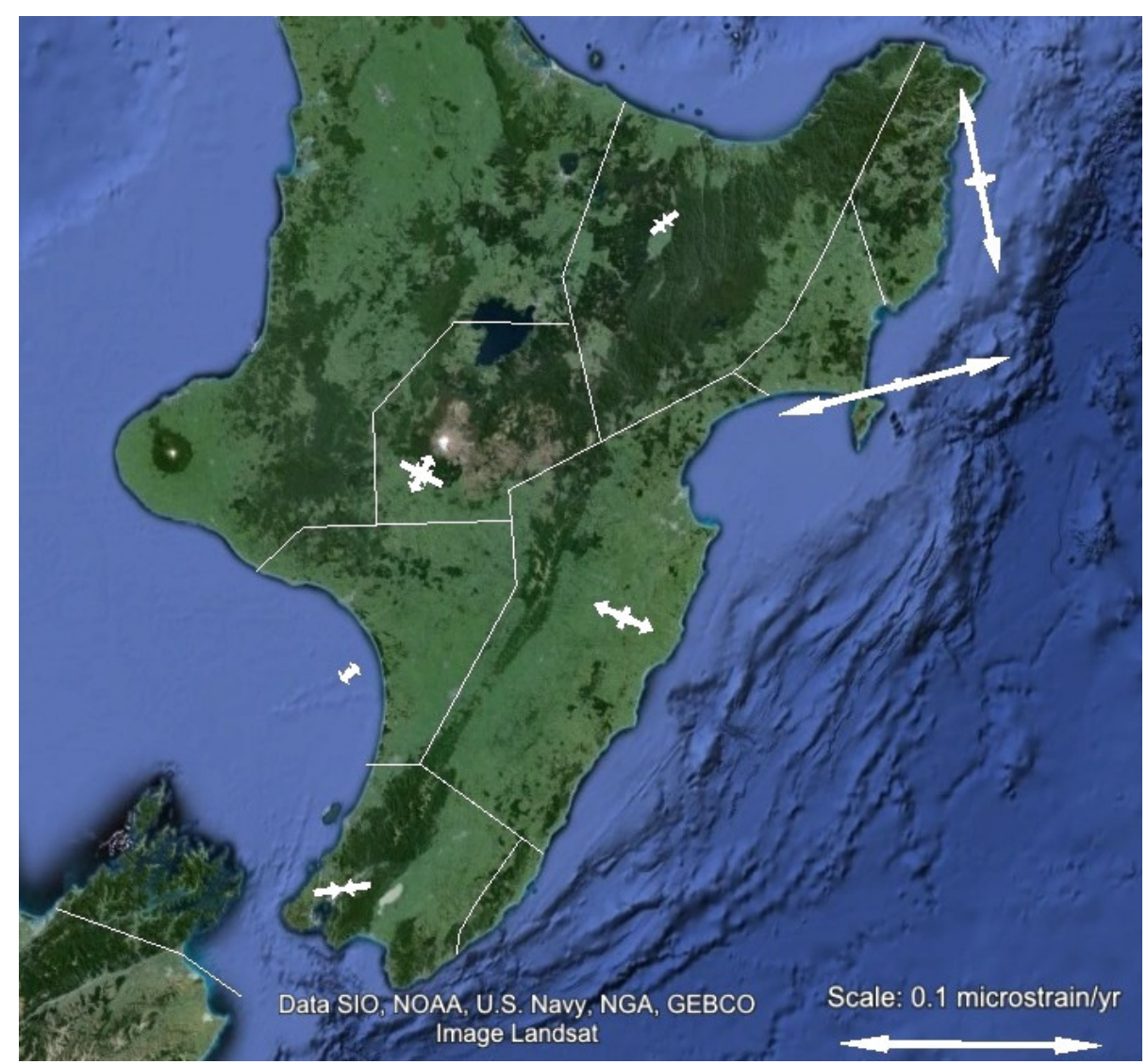

Figure 3.3e: Direction and magnitude representations of principal strain rates in 7 regions, derived from the equivalent average SSE secular velocities. Background $\odot$ GoogleEarth.

Uncertainties for strains and rotations shown in Appendix 12 were empirically derived by Monte Carlo simulation [Cox and Siebert, 2006; Papadopoulis and Yeung, 2001]. Marjetič et al. [2010] have shown that this is an appropriate method for determining the statistical properties of strain and rotation tensors in a geodetic network, but it should be noted that this method only applies to the specific combination of sites used in each region, and, as observed above, changes in the selection of sites can have much greater effects on the results than these uncertainty values imply.

Appendix 12 also includes an analysis of the displacement field for all the relevant cGPS sites, i.e. treating the whole of the NE to SW zone of the North Island as one region, for comparison with other published results. 


\subsection{SSE catalogue}

The preliminary SSE catalogue is shown in table 3.4. The catalogue is considered preliminary because procedures for optimizing the slip area are still under development. Nevertheless, the locations and moment values are considered precise within the $95 \%$ confidence limits quoted, subject to the conditions indicated below.

The following points require noting:

- It seems unlikely that the source slips that arise from the modelling in the Volcanic area are necessarily SSEs (note V in table), although transient fault-slip-like behaviour has been observed in other rift settings [Grandin et al., 2009; Rowland et al., 2010]. Since the model is constrained by source faults located on the subduction interface, it is feasible that the depths used in deriving the slip values are incorrect for the 8 events identified, which means that the source sizes and magnitudes could be over-estimated if the events occurred in the over-riding plate, or comprised some other sort of deformation transient.

- Events 141, 143 and 144 are directly associated with the 2013 Cook Strait earthquake sequence. (note $\mathrm{E}$ in table).

- Events 71 and 77 could not be satisfactorily constrained in the inversion and their results are considered unreliable and were this excluded from further analysis. (note $\mathrm{U}$ in table)

- Two 'negative' E-component surface events were 'absorbed' during the inversions, but 4 new 'negative' dip-slip sources (i.e. hanging wall slipping downdip rather than updip) appeared from the inversions ( 2 of which were not significantly different to zero at the 0.05 level). There are, therefore, a total of 46 'negative' dip-slip source slips in the catalogue, of which 42 are outside the volcanic area, that cannot be currently explained as SSEs occurring on the subduction interface (this is discussed further in 5.2.3). It is therefore feasible that all or some depths used in deriving these slip values are incorrect, and hence the source sizes and magnitudes could also be over-estimated.

- Fig. 3.4 shows the distribution and preliminary areas and slips for the 139 non-volcanic and non-excluded events, colour-coded to show those for which the location 95\% confidence interval boundary is well-defined (dark blue); and those for which the boundary does not close or is ill-defined or where there could be multiple minima (pale blue).

- In judging the confidence that can be associated with each source event, due consideration must be given to the following factors:

o whether the location confidence boundary is poorly-constrained (indicated by 999 values in the $95 \%$ CI columns)

- the ratio of the slip magnitudes to their confidence intervals

$\circ$ the ratio of the moment confidence interval to the mean moment

- the overall RMS fit residual value, and

$\circ$ the number of sites at which the surface events were detected 
- cGPS site PAEK has been removed from the inversion for event 1 since it only commenced operation just before or coincident with the onset of the event and therefore does not have a stable antecedent period (note $\mathrm{P}$ in table).

- An additional 'null' site was inserted into the displacement database for events 104 and 136 in order to better constrain the inversions (note $\mathrm{N}$ in table). These two events' 'sites' values are therefore overstated by 1 , and the 'nulls' understated by 1 . There is therefore a small error in the confidence limit estimates due to the use of slightly incorrect values of the degrees of freedom.

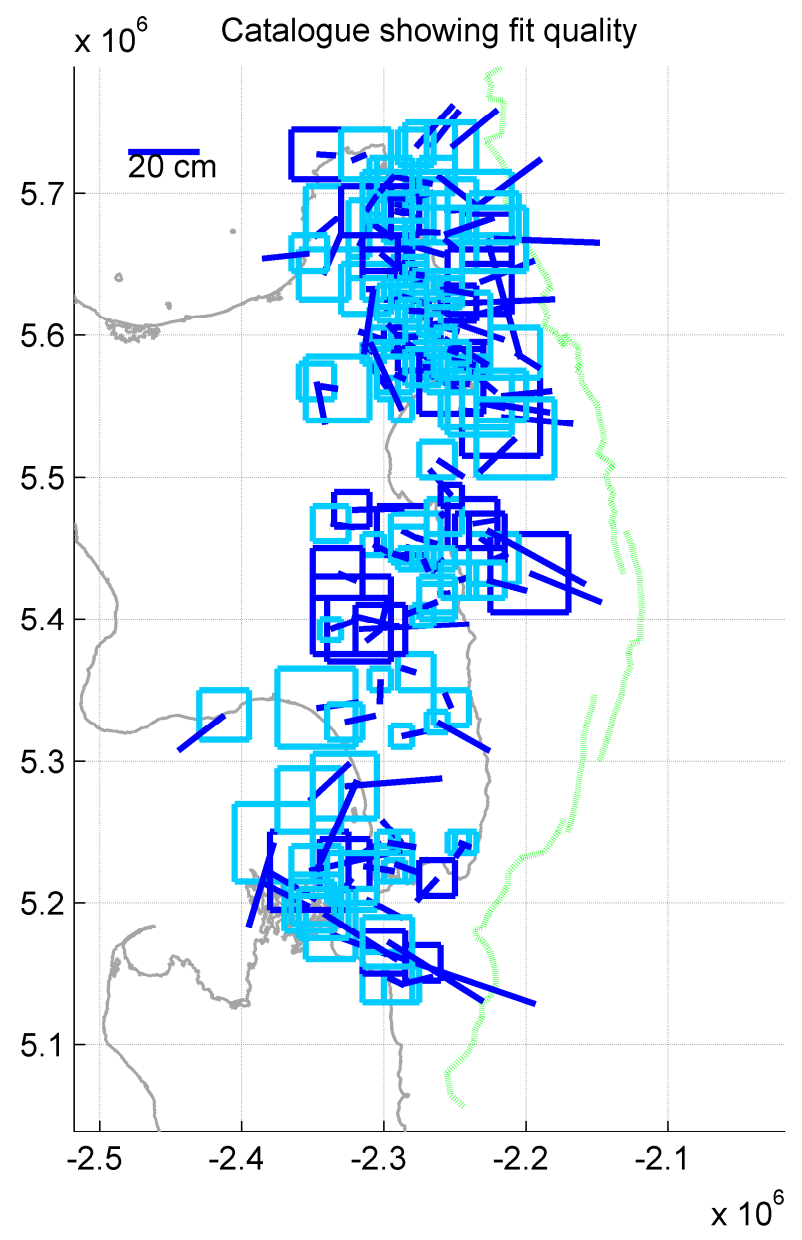

Figure 3.4: Surface projection (NZTM rotated by $42^{\circ}$ ) of slip areas and slip vectors from inversions for non-volcanic events. Dark blue boundaries indicate events that are well-constrained and for which $95 \%$ confidence limits can be assigned to the location, whereas pale blue boundaries refer to events that are not well-constrained. Events 71, 77 and 143 omitted. The green line indicates the location of the trench. 
Table 3.4: Preliminary catalogue of Hikurangi SSEs from Jan 2000 to Feb 2014

\begin{tabular}{|c|c|c|c|c|c|c|c|c|c|c|c|c|c|c|c|c|c|c|c|c|c|c|c|c|}
\hline Event & $\begin{array}{l}\text { days } \\
\text { from } \\
2000\end{array}$ & year & month & day & $\begin{array}{c}\text { duration } \\
\text { (days) }\end{array}$ & lat & $\begin{array}{c}95 \% \mathrm{Cl} \\
(\mathrm{km})\end{array}$ & long & $\begin{array}{c}95 \% \mathrm{Cl} \\
(\mathrm{km})\end{array}$ & $\begin{array}{l}\text { depth } \\
(\mathrm{km})\end{array}$ & $\begin{array}{c}\text { slip(ss) } \\
(\mathrm{cm})\end{array}$ & $95 \% \mathrm{Cl}$ & $\begin{array}{l}\text { slip(ds) } \\
\mathrm{cm}\end{array}$ & $95 \% \mathrm{Cl}$ & $\begin{array}{c}\text { moment } \\
\text { (N.m) }\end{array}$ & $95 \% \mathrm{Cl}$ & $\mathrm{Mw}$ & $95 \% \mathrm{Cl}-$ & $95 \% \mathrm{Cl}+$ & sites & nulls & $\begin{array}{c}\text { rms fit } \\
(\mathrm{mm})\end{array}$ & $\begin{array}{c}\text { fault area } \\
\left(\mathrm{km}^{\wedge} 2\right)\end{array}$ & $\begin{array}{c}\text { Note: } \\
\text { see } \\
\text { text }\end{array}$ \\
\hline 1 & 108 & 2000 & 4 & 17 & 134 & -41.34 & 999 & 174.1 & 999 & 39 & -5.6 & 53.2 & 15.9 & 34.3 & $1.61 E+19$ & $1.63 \mathrm{E}+19$ & 6.7 & 5.4 & 6.9 & 3 & 0 & 1.1 & 1397 & $P$ \\
\hline 2 & 1008 & 2002 & 10 & 4 & 56 & -38.63 & 999 & 178.01 & 999 & 15 & -4.7 & 0.1 & 11.2 & 0.2 & $6.14 \mathrm{E}+18$ & $1.28 \mathrm{E}+17$ & 6.5 & 6.5 & 6.5 & 1 & 1 & 0.1 & 1261 & \\
\hline 3 & 1242 & 2003 & 5 & 26 & 198 & -41.06 & 48 & 174.58 & 50 & 33 & 6.1 & 2.6 & 10.2 & 1.1 & $6.49 \mathrm{E}+18$ & $1.6 \mathrm{E}+18$ & 6.5 & 6.4 & 6.5 & 5 & 4 & 2.1 & 1356 & \\
\hline 4 & 1279 & 2003 & 7 & 2 & 15 & -38.76 & 999 & 177.95 & 999 & 14 & 0.1 & 0.0 & 2.6 & 0.0 & $1.32 E+18$ & $4.24 E+16$ & 6.0 & 6.0 & 6.0 & 1 & 3 & 0.0 & 1257 & \\
\hline 5 & 1323 & 2003 & 8 & 15 & 42 & -39.67 & 2 & 176.72 & 12 & 22 & -2.5 & 0.2 & 4.7 & 0.2 & $2.67 E+18$ & $1.53 \mathrm{E}+17$ & 6.2 & 6.2 & 6.2 & 1 & 3 & 0.1 & 1265 & \\
\hline 6 & 1492 & 2004 & 1 & 31 & 295 & -40.19 & 999 & 175.25 & 999 & 45 & 1.6 & 5.7 & 11.8 & 3.9 & $1.64 E+19$ & $7.06 \mathrm{E}+18$ & 6.7 & 6.6 & 6.9 & 9 & 3 & 2.5 & 3386 & \\
\hline 7 & 1493 & 2004 & 2 & 1 & 513 & -41.86 & 4 & 174.54 & 3 & 10 & 3.4 & 0.5 & -7.2 & 0.2 & $2.06 \mathrm{E}+18$ & $8.46 \mathrm{E}+16$ & 6.1 & 6.1 & 6.2 & 1 & 3 & 0.1 & 646 & \\
\hline 8 & 1499 & 2004 & 2 & 7 & 29 & -38.17 & 999 & 178.11 & 999 & 21 & -4.5 & 3.3 & 16.0 & 8.8 & $2.1 \mathrm{E}+19$ & $1.47 \mathrm{E}+19$ & 6.8 & 6.5 & 7.0 & 2 & 1 & 0.9 & 3191 & \\
\hline 9 & 1505 & 2004 & 2 & 13 & 15 & -39.62 & 999 & 176.47 & 999 & 28 & -4.4 & 3.4 & 8.6 & 2.2 & $9.35 E+17$ & $3.08 \mathrm{E}+17$ & 5.9 & 5.8 & 6.0 & 1 & 3 & 0.3 & 235 & \\
\hline 10 & 1590 & 2004 & 5 & 8 & 21 & -39.76 & 999 & 176.77 & 999 & 20 & -1.8 & 1.2 & 5.9 & 1.6 & $3.12 E+18$ & $2.04 \mathrm{E}+18$ & 6.3 & 6.0 & 6.4 & 1 & 3 & 1.1 & 1260 & \\
\hline 11 & 1591 & 2004 & 5 & 9 & 21 & -38.15 & 999 & 178.35 & 999 & 14 & 0.2 & 0.3 & 2.6 & 0.2 & $1.33 E+18$ & $2.25 \mathrm{E}+17$ & 6.0 & 6.0 & 6.1 & 1 & 3 & 0.3 & 1272 & \\
\hline 12 & 1613 & 2004 & 5 & 31 & 23 & -38.52 & 999 & 177.84 & 999 & 20 & -3.2 & 3.1 & -3.0 & 1.2 & $1.19 E+18$ & $1.19 \mathrm{E}+18$ & 6.0 & 4.3 & 6.2 & 2 & 3 & 1.2 & 653 & \\
\hline 13 & 1761 & 2004 & 10 & 26 & 151 & -41.3 & 999 & 174.86 & 999 & 18 & 1.4 & 2.1 & -8.8 & 2.4 & $8.53 E+17$ & 3.44E+17 & 5.9 & 5.7 & 6.0 & 6 & 6 & 1.6 & 238 & \\
\hline 14 & 1762 & 2004 & 10 & 27 & 40 & -38.77 & 25 & 178.24 & 21 & 9 & 1.2 & 13.3 & 27.1 & 3.5 & $7.26 \mathrm{E}+18$ & $4.38 \mathrm{E}+18$ & 6.5 & 6.2 & 6.6 & 1 & 3 & 1.6 & 635 & \\
\hline 15 & 1801 & 2004 & 12 & 5 & 37 & -38.26 & 999 & 178.61 & 999 & 9 & 0.3 & 6.3 & 3.9 & 1.8 & $4.28 \mathrm{E}+18$ & $4.59 \mathrm{E}+18$ & 6.4 & 5.6 & 6.6 & 2 & 3 & 2.7 & 2059 & \\
\hline 16 & 1803 & 2004 & 12 & 7 & 217 & -39.98 & 47 & 175.93 & 58 & 33 & 1.5 & 8.6 & 31.0 & 3.2 & $2.67 E+19$ & $7.88 \mathrm{E}+18$ & 6.9 & 6.8 & 7.0 & 8 & 8 & 4.8 & 2159 & \\
\hline 17 & 1847 & 2005 & 1 & 20 & 25 & -40.66 & 999 & 174.25 & 999 & 63 & -23.8 & 15.3 & -7.2 & 3.3 & $3.76 E+19$ & $2.83 \mathrm{E}+19$ & 7.0 & 6.6 & 7.2 & 3 & 3 & 1.5 & 3692 & \\
\hline 18 & 1860 & 2005 & 2 & 2 & 116 & -38.85 & 32 & 177.72 & 30 & 17 & 0.5 & 0.7 & -7.1 & 1.3 & $1.84 \mathrm{E}+18$ & $5.14 \mathrm{E}+17$ & 6.1 & 6.0 & 6.2 & 2 & 3 & 0.6 & 644 & \\
\hline 19 & 1899 & 2005 & 3 & 13 & 38 & -38.03 & 18 & 178.33 & 25 & 17 & -0.8 & 0.4 & 5.5 & 0.6 & $1.46 \mathrm{E}+18$ & $3.86 \mathrm{E}+17$ & 6.0 & 6.0 & 6.1 & 1 & 3 & 0.4 & 657 & \\
\hline 20 & 2015 & 2005 & 7 & 7 & 50 & -38.41 & 999 & 178.02 & 999 & 17 & 10.7 & 5.4 & 2.4 & 8.0 & $1.12 E+18$ & $7.29 \mathrm{E}+17$ & 6.0 & 5.7 & 6.1 & 3 & 3 & 1.4 & 234 & \\
\hline 21 & 2231 & 2006 & 2 & 8 & 29 & -38.7 & 999 & 177.94 & 999 & 15 & -0.3 & 0.7 & 2.8 & 0.6 & $7.28 \mathrm{E}+17$ & 4.47E+17 & 5.8 & 5.6 & 6.0 & 1 & 3 & 0.5 & 643 & \\
\hline 22 & 2261 & 2006 & 3 & 10 & 24 & -38.06 & 999 & 178.3 & 999 & 17 & -1.0 & 2.6 & 5.3 & 2.3 & $1.48 \mathrm{E}+18$ & $1.07 E+18$ & 6.0 & 5.7 & 6.2 & 2 & 3 & 2.0 & 657 & \\
\hline 23 & 2309 & 2006 & 4 & 27 & 163 & -41.19 & 999 & 175.06 & 999 & 18 & 6.5 & 2.9 & -5.6 & 7.3 & $8.65 E+17$ & $8.14 E+17$ & 5.9 & 5.1 & 6.1 & 9 & 9 & 2.5 & 234 & \\
\hline 24 & 2338 & 2006 & 5 & 26 & 32 & -38.1 & 999 & 177.9 & 999 & 27 & 7.6 & 2.8 & 3.7 & 6.9 & $9.12 E+17$ & $7.03 E+17$ & 5.9 & 5.5 & 6.1 & 1 & 3 & 0.5 & 242 & \\
\hline 25 & 2343 & 2006 & 5 & 31 & 46 & -39.92 & 42 & 177.21 & 23 & 12 & 1.1 & 1.1 & 7.0 & 0.9 & $3.58 \mathrm{E}+18$ & $6.23 \mathrm{E}+17$ & 6.3 & 6.3 & 6.4 & 9 & 9 & 1.7 & 1243 & \\
\hline 26 & 2372 & 2006 & 6 & 29 & 23 & -38.87 & 48 & 178.21 & 28 & 9 & 3.6 & 3.5 & 13.0 & 1.9 & $6.83 \mathrm{E}+18$ & $1.73 \mathrm{E}+18$ & 6.5 & 6.4 & 6.6 & 3 & 3 & 1.4 & 1241 & \\
\hline 27 & 2382 & 2006 & 7 & 9 & 8 & -40.17 & 999 & 176.41 & 999 & 20 & 1.8 & 2.2 & -2.5 & 1.1 & $3.13 E+17$ & $2.34 \mathrm{E}+17$ & 5.6 & 5.2 & 5.8 & 1 & 3 & 0.6 & 230 & \\
\hline 28 & 2397 & 2006 & 7 & 24 & 193 & -38.94 & 999 & 177.77 & 999 & 15 & 7.6 & 1.4 & -10.1 & 4.5 & $3.21 E+18$ & $1.63 \mathrm{E}+18$ & 6.3 & 6.1 & 6.4 & 3 & 3 & 1.7 & 641 & \\
\hline 29 & 2413 & 2006 & 8 & 9 & 50 & -40.39 & 24 & 177.32 & 28 & 7 & -8.3 & 5.8 & 20.2 & 2.3 & $2.71 E+19$ & $3.5 E+18$ & 6.9 & 6.9 & 6.9 & 14 & 14 & 2.1 & 3042 & \\
\hline 30 & 2437 & 2006 & 9 & 2 & 14 & -38.07 & 999 & 178.5 & 999 & 12 & 1.3 & 0.9 & 4.9 & 0.9 & $4.76 \mathrm{E}+17$ & $6.07 E+16$ & 5.7 & 5.7 & 5.8 & 1 & 3 & 0.1 & 233 & \\
\hline 31 & 2447 & 2006 & 9 & 12 & 29 & -39.15 & 999 & 176 & 999 & 62 & -4.8 & 9.4 & -22.5 & 4.9 & $2.55 E+18$ & $1.83 \mathrm{E}+18$ & 6.2 & 5.8 & 6.4 & 1 & 3 & 0.5 & 272 & V \\
\hline 32 & 2505 & 2006 & 11 & 9 & 61 & -38.69 & 9 & 177.66 & 8 & 20 & 0.6 & 0.7 & 6.5 & 0.7 & $6.14 \mathrm{E}+17$ & $7.97 E+16$ & 5.8 & 5.8 & 5.8 & 1 & 3 & 0.2 & 234 & \\
\hline 33 & 2514 & 2006 & 11 & 18 & 70 & -39.27 & 999 & 175.64 & 999 & 74 & -12.7 & 6.7 & -15.4 & 1.5 & $2.03 E+19$ & $9.51 \mathrm{E}+18$ & 6.8 & 6.6 & 6.9 & 4 & 4 & 1.4 & 2581 & V \\
\hline 34 & 2519 & 2006 & 11 & 23 & 254 & -41.32 & 999 & 174.53 & 999 & 25 & -5.3 & 18.1 & 10.3 & 12.0 & $1.66 \mathrm{E}+18$ & $1.23 \mathrm{E}+18$ & 6.1 & 5.7 & 6.2 & 7 & 7 & 2.3 & 250 & \\
\hline 35 & 2591 & 2007 & 2 & 3 & 24 & -37.86 & 999 & 178.43 & 999 & 18 & -2.3 & 2.1 & 12.7 & 4.7 & $3.4 \mathrm{E}+18$ & $1.97 \mathrm{E}+18$ & 6.3 & 6.0 & 6.4 & 2 & 3 & 1.0 & 664 & \\
\hline 36 & 2657 & 2007 & 4 & 10 & 39 & -38.06 & 999 & 178.3 & 999 & 17 & -0.2 & 1.6 & 5.0 & 1.7 & $1.33 E+18$ & $5.06 \mathrm{E}+17$ & 6.0 & 5.9 & 6.1 & 3 & 3 & 0.9 & 657 & \\
\hline 37 & 2729 & 2007 & 6 & 21 & 51 & -41.42 & 999 & 175.43 & 999 & 9 & -1.4 & 0.6 & 3.5 & 1.3 & $3.45 \mathrm{E}+17$ & $1.5 \mathrm{E}+17$ & 5.6 & 5.5 & 5.7 & 3 & 3 & 0.6 & 228 & \\
\hline 38 & 2738 & 2007 & 6 & 30 & 26 & -39.01 & 999 & 177.7 & 999 & 15 & -5.1 & 1.0 & 9.1 & 1.2 & $9.62 E+17$ & $2.63 \mathrm{E}+17$ & 5.9 & 5.8 & 6.0 & 1 & 3 & 0.3 & 230 & \\
\hline
\end{tabular}




\begin{tabular}{|c|c|c|c|c|c|c|c|c|c|c|c|c|c|c|c|c|c|c|c|c|c|c|c|c|}
\hline Event & $\begin{array}{l}\text { days } \\
\text { from } \\
2000 \\
\end{array}$ & year & month & day & $\begin{array}{l}\text { duration } \\
\text { (days) }\end{array}$ & lat & $\begin{array}{c}95 \% \mathrm{Cl} \\
(\mathrm{km}) \\
\end{array}$ & long & $\begin{array}{c}95 \% \mathrm{Cl} \\
(\mathrm{km}) \\
\end{array}$ & $\begin{array}{c}\text { depth } \\
(\mathrm{km})\end{array}$ & $\begin{array}{c}\text { slip(ss) } \\
(\mathrm{cm})\end{array}$ & $95 \% \mathrm{Cl}$ & $\begin{array}{c}\text { slip(ds) } \\
\mathrm{cm}\end{array}$ & $95 \% \mathrm{Cl}$ & $\begin{array}{c}\text { moment } \\
\text { (N.m) }\end{array}$ & $95 \% \mathrm{Cl}$ & $\mathrm{Mw}$ & $95 \% \mathrm{Cl}-$ & $95 \% \mathrm{Cl}+$ & sites & nulls & $\begin{array}{c}\text { rms fit } \\
(\mathrm{mm})\end{array}$ & $\begin{array}{c}\text { fault area } \\
\left(\mathrm{km}^{\wedge} 2\right)\end{array}$ & $\begin{array}{l}\text { Note: } \\
\text { see } \\
\text { text }\end{array}$ \\
\hline \begin{tabular}{l|}
39 \\
\end{tabular} & 2802 & 2007 & 9 & 2 & 39 & -39.07 & 999 & \begin{tabular}{ll|}
177.71 \\
\end{tabular} & 999 & 14 & $\begin{array}{l}0.7 \\
\end{array}$ & \begin{tabular}{|l|}
3.6 \\
\end{tabular} & $\begin{array}{l}8.7 \\
\end{array}$ & 2.5 & $8.15 E+17$ & $3.7 E+17$ & 5.9 & \begin{tabular}{|l|}
5.7 \\
\end{tabular} & \begin{tabular}{|l|}
6.0 \\
\end{tabular} & 4 & 4 & 1.6 & 230 & \\
\hline 40 & 2821 & 2007 & 9 & 21 & 22 & -38.05 & 999 & 178.09 & 999 & 22 & -3.8 & 1.6 & 5.7 & 0.6 & $6.69 \mathrm{E}+17$ & $9.17 E+16$ & 5.8 & 5.8 & 5.9 & 1 & 3 & 0.2 & 241 & \\
\hline 41 & 2823 & 2007 & 9 & 23 & 419 & -40.95 & 31 & 174.32 & 36 & 47 & 5.8 & 3.8 & 26.0 & 2.4 & $3.75 \mathrm{E}+19$ & $6.55 \mathrm{E}+18$ & 7.0 & 6.9 & 7.0 & 13 & 13 & 5.3 & 3497 & \\
\hline 42 & 2842 & 2007 & 10 & 12 & 16 & -38.68 & 999 & 177.45 & 999 & 25 & 2.7 & 7.4 & -3.0 & 3.1 & $5.57 \mathrm{E}+17$ & $4.76 \mathrm{E}+17$ & 5.8 & 5.2 & 5.9 & 4 & 4 & 1.0 & 236 & \\
\hline 43 & 2872 & 2007 & 11 & 11 & 159 & -39.34 & 48 & 175.94 & 53 & 55 & -13.3 & 4.0 & -9.4 & 5.5 & $4.89 \mathrm{E}+18$ & $1.28 \mathrm{E}+18$ & 6.4 & 6.3 & 6.5 & 11 & 11 & 1.7 & 733 & V \\
\hline 44 & 2904 & 2007 & 12 & 13 & 50 & -38.75 & 33 & 178.48 & 53 & 7 & 6.0 & 5.6 & 15.4 & 2.6 & $1.37 E+19$ & $4.89 \mathrm{E}+18$ & 6.7 & 6.6 & 6.8 & 5 & 5 & 2.8 & 2041 & \\
\hline 45 & 2953 & 2008 & 1 & 31 & 15 & -38.06 & 11 & 178.3 & 16 & 17 & -0.4 & 0.2 & 2.0 & 0.2 & $1.06 \mathrm{E}+18$ & $2.15 \mathrm{E}+17$ & 6.0 & 5.9 & 6.0 & 1 & 3 & 0.3 & 1287 & \\
\hline 46 & 2966 & 2008 & 2 & 13 & 26 & -40.01 & 15 & 177.18 & 13 & 11 & -2.5 & 0.7 & 3.4 & 0.7 & $2.1 \mathrm{E}+18$ & $4.01 E+17$ & 6.1 & 6.1 & 6.2 & 6 & 6 & 0.8 & 1241 & \\
\hline 47 & 2978 & 2008 & 2 & 25 & 119 & -37.87 & 999 & 177.91 & 999 & 34 & -5.5 & 1.9 & -6.7 & 1.4 & $7.92 \mathrm{E}+18$ & $2.18 \mathrm{E}+18$ & 6.5 & 6.4 & 6.6 & 6 & 6 & 1.6 & 2271 & \\
\hline 48 & 2982 & 2008 & 2 & 29 & 158 & -40.07 & 25 & 176.06 & 28 & 28 & -3.3 & 1.0 & -4.1 & 1.0 & $2.68 \mathrm{E}+18$ & $6.18 \mathrm{E}+17$ & 6.2 & 6.1 & 6.3 & 15 & 15 & 2.0 & 1275 & \\
\hline 49 & 2988 & 2008 & 3 & 6 & 10 & -38.76 & 999 & 177.95 & 999 & 14 & -2.1 & 1.8 & 8.2 & 1.7 & $8 \mathrm{E}+17$ & $3.19 E+17$ & 5.9 & 5.7 & 6.0 & 2 & 3 & 0.7 & 231 & \\
\hline 50 & 2989 & 2008 & 3 & 7 & 37 & -39.13 & 19 & 177.8 & 13 & 12 & -0.7 & 0.8 & 5.2 & 0.7 & $4.35 E+18$ & $6.25 E+17$ & 6.4 & 6.3 & 6.4 & 6 & 6 & 2.1 & 2061 & \\
\hline 51 & 3020 & 2008 & 4 & 7 & 85 & -39.35 & 999 & 175.4 & 999 & 80 & 5.7 & 9.7 & 5.4 & 2.8 & $5.7 \mathrm{E}+18$ & $6.74 E+18$ & 6.4 & 5.9 & 6.7 & 11 & 11 & 3.7 & 1624 & V \\
\hline 52 & 3040 & 2008 & 4 & 27 & 27 & -39.77 & 999 & 176.69 & 999 & 21 & -4.3 & 1.3 & -0.9 & 1.6 & $1.16 \mathrm{E}+18$ & $5.03 E+17$ & 6.0 & 5.8 & 6.1 & 4 & 4 & 1.4 & 644 & \\
\hline 53 & 3046 & 2008 & 5 & 3 & 21 & -39.84 & 999 & 176.62 & 999 & 21 & 1.6 & 2.0 & -4.6 & 2.3 & $4.65 E+17$ & $3.09 E+17$ & 5.7 & 5.4 & 5.9 & 2 & 3 & 0.9 & 232 & \\
\hline 54 & 3069 & 2008 & 5 & 26 & 32 & -39.3 & 999 & 177.7 & 999 & 12 & -0.4 & 2.0 & 6.1 & 3.6 & $1.59 E+18$ & $1.06 \mathrm{E}+18$ & 6.1 & 5.8 & 6.2 & 7 & 7 & 2.1 & 635 & \\
\hline 55 & 3093 & 2008 & 6 & 19 & 123 & -39.42 & 999 & 175.33 & 999 & 80 & 10.1 & 12.0 & -12.5 & 6.2 & $1.16 \mathrm{E}+19$ & $5.35 E+18$ & 6.6 & 6.5 & 6.8 & 10 & 10 & 4.2 & 1613 & v \\
\hline 56 & 3113 & 2008 & 7 & 9 & 34 & -39.08 & 23 & 178 & 27 & 10 & -0.1 & 0.4 & 2.4 & 0.5 & $6.27 \mathrm{E}+17$ & $1.57 E+17$ & 5.8 & 5.7 & 5.9 & 3 & 3 & 0.7 & 634 & \\
\hline 57 & 3126 & 2008 & 7 & 22 & 35 & -37.47 & 12 & 178.09 & 11 & 42 & -0.3 & 1.6 & 5.4 & 0.2 & $3.1 \mathrm{E}+18$ & $1.1 \mathrm{E}+18$ & 6.3 & 6.1 & 6.4 & 1 & 3 & 0.2 & 1402 & \\
\hline 58 & 3140 & 2008 & 8 & 5 & 47 & -39.98 & 999 & 176.84 & 999 & 16 & -3.9 & 2.9 & -3.8 & 1.7 & $4.99 \mathrm{E}+17$ & $3.33 \mathrm{E}+17$ & 5.7 & 5.4 & 5.9 & 5 & 5 & 1.3 & 230 & \\
\hline 59 & 3147 & 2008 & 8 & 12 & 42 & -38.4 & 999 & 178.47 & 999 & 10 & -3.2 & 0.8 & 3.8 & 1.5 & $4.12 E+18$ & $1.68 \mathrm{E}+18$ & 6.3 & 6.2 & 6.4 & 3 & 3 & 1.9 & 2061 & \\
\hline 60 & 3158 & 2008 & 8 & 23 & 80 & -41.78 & 999 & 174.23 & 999 & 19 & -2.3 & 2.0 & 4.7 & 1.4 & $2.82 E+18$ & $9.02 E+17$ & 6.2 & 6.1 & 6.3 & 3 & 3 & 1.4 & 1331 & \\
\hline 61 & 3165 & 2008 & 8 & 30 & 145 & -39.12 & 999 & 177.96 & 999 & 10 & -0.5 & 1.1 & -3.3 & 2.2 & $3.31 \mathrm{E}+17$ & $2.39 \mathrm{E}+17$ & 5.6 & 5.2 & 5.8 & 2 & 3 & 1.3 & 228 & \\
\hline 62 & 3202 & 2008 & 10 & 6 & 73 & -40.16 & 999 & 176.58 & 999 & 17 & -3.7 & 3.2 & -9.0 & 2.3 & $2.5 \mathrm{E}+18$ & $1.31 \mathrm{E}+18$ & 6.2 & 6.0 & 6.3 & 2 & 3 & 3.4 & 638 & \\
\hline 63 & 3242 & 2008 & 11 & 15 & 73 & -37.95 & 59 & 178.12 & 40 & 25 & 5.5 & 2.0 & 3.7 & 0.5 & $3.52 \mathrm{E}+18$ & $1.32 \mathrm{E}+18$ & 6.3 & 6.2 & 6.4 & 6 & 6 & 1.2 & 1326 & \\
\hline 64 & 3295 & 2009 & 1 & 7 & 23 & -40.18 & 999 & 177.16 & 999 & 10 & 1.5 & 3.3 & 3.9 & 1.1 & $2.21 \mathrm{E}+18$ & $1.68 \mathrm{E}+18$ & 6.2 & 5.8 & 6.3 & 5 & 5 & 1.6 & 1238 & \\
\hline 65 & 3328 & 2009 & 2 & 9 & 330 & -41.26 & 999 & 174.9 & 999 & 19 & -2.8 & 1.5 & 7.5 & 2.8 & $2.09 E+18$ & $9.61 \mathrm{E}+17$ & 6.1 & 6.0 & 6.3 & 18 & 18 & 3.4 & 657 & \\
\hline 66 & 3340 & 2009 & 2 & 21 & 28 & -39.89 & 999 & 176.71 & 999 & 19 & -3.5 & 2.6 & 1.5 & 2.4 & $3.97 \mathrm{E}+17$ & $3.63 E+17$ & 5.7 & 5.0 & 5.9 & 2 & 3 & 1.2 & 231 & \\
\hline 67 & 3390 & 2009 & 4 & 12 & 35 & -38.95 & 999 & 177.69 & 999 & 16 & 0.0 & 0.7 & 2.7 & 0.7 & $6.95 E+17$ & $2.72 \mathrm{E}+17$ & 5.8 & 5.7 & 5.9 & 2 & 3 & 1.2 & 642 & \\
\hline 68 & 3399 & 2009 & 4 & 21 & 10 & -38.67 & 999 & 178.27 & 999 & 10 & -0.2 & 0.9 & -3.1 & 0.8 & $2.82 E+17$ & $2.32 \mathrm{E}+17$ & 5.6 & 5.1 & 5.7 & 1 & 3 & 0.3 & 229 & \\
\hline 69 & 3414 & 2009 & 5 & 6 & 35 & -40.12 & 999 & 176.61 & 999 & 17 & 0.4 & 1.3 & 3.7 & 1.4 & $9.78 \mathrm{E}+17$ & $8.72 E+17$ & 5.9 & 5.3 & 6.1 & 1 & 3 & 2.0 & 638 & \\
\hline 70 & 3419 & 2009 & 5 & 11 & 55 & -38.35 & 30 & 177.93 & 25 & 21 & -0.3 & 0.7 & 2.6 & 0.6 & $1.38 \mathrm{E}+18$ & $4.9 \mathrm{E}+17$ & 6.0 & 5.9 & 6.1 & 2 & 3 & 0.8 & 1286 & \\
\hline 71 & 3427 & 2009 & 5 & 19 & 61 & -38.66 & 999 & 176.96 & 999 & 42 & -3.3 & 1.3 & -5.9 & 0.5 & $3.81 \mathrm{E}+18$ & $1.11 \mathrm{E}+18$ & 6.3 & 6.2 & 6.4 & 2 & 3 & 0.3 & 1403 & U \\
\hline 72 & 3461 & 2009 & 6 & 22 & 35 & -40.14 & 999 & 176.82 & 999 & 14 & 0.8 & 0.9 & 2.9 & 2.3 & $8.23 E+17$ & $4.09 E+17$ & 5.9 & 5.7 & 6.0 & 8 & 8 & 1.9 & 636 & \\
\hline 73 & 3464 & 2009 & 6 & 25 & 11 & -39.09 & 999 & 177.92 & 999 & 11 & 0.1 & 1.0 & 4.5 & 0.9 & $4.16 \mathrm{E}+17$ & $2.51 \mathrm{E}+17$ & 5.7 & 5.4 & 5.8 & 1 & 3 & 0.7 & 229 & \\
\hline 74 & 3464 & 2009 & 6 & 25 & 39 & -37.96 & 999 & 178.32 & 999 & 19 & -0.4 & 0.6 & 4.9 & 0.9 & $2.58 \mathrm{E}+18$ & $7.95 E+17$ & 6.2 & 6.1 & 6.3 & 2 & 3 & 0.9 & 1298 & \\
\hline 75 & 3479 & 2009 & 7 & 10 & 30 & -38.43 & 999 & 178.07 & 999 & 16 & 2.2 & 1.0 & 2.4 & 0.5 & $8.31 E+17$ & $3.43 E+17$ & 5.9 & 5.7 & 6.0 & 9 & 9 & 1.2 & 648 & \\
\hline 76 & 3497 & 2009 & 7 & 28 & 36 & -38.91 & 999 & 177.36 & 999 & 23 & 7.0 & 2.4 & -5.0 & 2.2 & $8.32 \mathrm{E}+17$ & $5.13 \mathrm{E}+17$ & 5.9 & 5.6 & 6.0 & 1 & 3 & 0.5 & 234 & \\
\hline
\end{tabular}




\begin{tabular}{|c|c|c|c|c|c|c|c|c|c|c|c|c|c|c|c|c|c|c|c|c|c|c|c|c|}
\hline Event & $\begin{array}{l}\text { days } \\
\text { from } \\
2000\end{array}$ & year & month & day & $\begin{array}{c}\text { duration } \\
\text { (days) }\end{array}$ & lat & $\begin{array}{c}95 \% \mathrm{Cl} \\
(\mathrm{km})\end{array}$ & long & $\begin{array}{c}95 \% \mathrm{Cl} \\
(\mathrm{km})\end{array}$ & $\begin{array}{l}\text { depth } \\
(\mathrm{km})\end{array}$ & $\begin{array}{c}\text { slip(ss) } \\
(\mathrm{cm})\end{array}$ & $95 \% \mathrm{Cl}$ & $\begin{array}{c}\text { slip(ds) } \\
\text { cm }\end{array}$ & $95 \% \mathrm{Cl}$ & $\begin{array}{c}\text { moment } \\
\text { (N.m) }\end{array}$ & $95 \% \mathrm{Cl}$ & $\mathrm{Mw}$ & $95 \% \mathrm{Cl}-$ & $95 \% \mathrm{Cl}_{+}$ & sites & nulls & $\begin{array}{c}\text { rms fit } \\
(\mathrm{mm})\end{array}$ & $\begin{array}{c}\text { fault area } \\
\left(\mathrm{km}^{\wedge} 2\right) \\
\end{array}$ & \begin{tabular}{|c|} 
Note: \\
see \\
text \\
\end{tabular} \\
\hline 77 & 3530 & 2009 & 8 & 30 & 33 & \begin{tabular}{|l|}
-38.08 \\
\end{tabular} & 999 & $\begin{array}{l}177.77 \\
\end{array}$ & \begin{tabular}{|l|l}
999 \\
\end{tabular} & 31 & $\begin{array}{l}-8.0 \\
\end{array}$ & 19.6 & \begin{tabular}{|l|}
3.4 \\
\end{tabular} & 3.6 & $1.44 \mathrm{E}+18$ & $2.34 E+18$ & 6.0 & 5.9 & 6.3 & 1 & 3 & 0.6 & 250 & $U$ \\
\hline 78 & 3578 & 2009 & 10 & 17 & 99 & -38.53 & 999 & 178.12 & 999 & 14 & -1.0 & 2.0 & -2.8 & 2.2 & $3.13 E+17$ & $1.64 \mathrm{E}+17$ & 5.6 & 5.4 & 5.7 & 9 & 9 & 1.7 & 231 & \\
\hline 79 & 3606 & 2009 & 11 & 14 & 69 & -39.62 & 20 & 176.09 & 42 & 39 & -2.2 & 1.1 & 5.3 & 1.0 & $3.11 E+18$ & $6.42 E+17$ & 6.3 & 6.2 & 6.3 & 9 & 9 & 1.7 & 1360 & \\
\hline 80 & 3611 & 2009 & 11 & 19 & 39 & -38.59 & 999 & 176.95 & 999 & 44 & -12.2 & 4.9 & 2.4 & 2.5 & $3.66 E+18$ & $1.26 \mathrm{E}+18$ & 6.3 & 6.2 & 6.4 & 6 & 6 & 1.3 & 722 & \\
\hline 81 & 3664 & 2010 & 1 & 11 & 59 & -39.5 & 46 & 177.94 & 42 & 8 & -1.9 & 2.6 & 20.2 & 2.8 & $2.47 E+19$ & $4.82 \mathrm{E}+18$ & 6.9 & 6.8 & 6.9 & 22 & 22 & 2.9 & 3048 & \\
\hline 82 & 3671 & 2010 & 1 & 18 & 12 & -38.64 & 999 & 177.57 & 999 & 24 & 2.0 & 3.1 & -6.1 & 1.7 & $6.24 E+17$ & $2.67 E+17$ & 5.8 & 5.6 & 5.9 & 12 & 12 & 1.3 & 236 & \\
\hline 83 & 3685 & 2010 & 2 & 1 & 113 & -39.32 & 999 & 175.81 & 999 & 62 & -4.0 & 4.1 & -9.8 & 2.3 & $6.27 E+18$ & $3.43 \mathrm{E}+18$ & 6.5 & 6.2 & 6.6 & 9 & 9 & 2.0 & 1476 & V \\
\hline 84 & 3686 & 2010 & 2 & 2 & 309 & -40.74 & 999 & 176.11 & 999 & 14 & 4.3 & 1.6 & -1.8 & 1.9 & $1.22 \mathrm{E}+18$ & $6.64 \mathrm{E}+17$ & 6.0 & 5.8 & 6.1 & 8 & 8 & 3.3 & 637 & \\
\hline 85 & 3689 & 2010 & 2 & 5 & 91 & -40.42 & 999 & 176.07 & 999 & 21 & 1.3 & 1.9 & -4.2 & 2.1 & $1.17 E+18$ & $5.81 \mathrm{E}+17$ & 6.0 & 5.8 & 6.1 & 24 & 24 & 2.0 & 643 & \\
\hline 86 & 3702 & 2010 & 2 & 18 & 74 & -38.88 & 999 & 178.13 & 999 & 10 & -4.4 & 1.4 & 12.8 & 1.5 & $1.12 E+19$ & $1.28 \mathrm{E}+18$ & 6.6 & 6.6 & 6.7 & 11 & 11 & 4.0 & 2056 & \\
\hline 87 & 3721 & 2010 & 3 & 9 & 18 & -37.67 & 999 & 178.4 & 999 & 24 & -1.7 & 1.1 & -4.2 & 0.8 & $2.43 E+18$ & $8.21 \mathrm{E}+17$ & 6.2 & 6.1 & 6.3 & 4 & 4 & 0.8 & 1340 & \\
\hline 88 & 3724 & 2010 & 3 & 12 & 13 & -39.77 & 999 & 177.06 & 999 & 15 & 1.5 & 0.7 & -1.2 & 0.9 & $4.9 \mathrm{E}+17$ & $3.14 \mathrm{E}+17$ & 5.7 & 5.4 & 5.9 & 6 & 6 & 0.8 & 638 & \\
\hline 89 & 3728 & 2010 & 3 & 16 & 49 & -38.56 & 33 & 178.74 & 28 & 5 & -33.6 & 10.3 & 9.4 & 6.4 & $1.75 E+19$ & $6.68 \mathrm{E}+18$ & 6.8 & 6.6 & 6.9 & 5 & 5 & 1.1 & 1230 & \\
\hline 90 & 3767 & 2010 & 4 & 24 & 52 & -39.24 & 999 & 178.35 & 999 & 5 & -4.3 & 17.7 & 6.9 & 7.4 & $6.26 \mathrm{E}+18$ & $5.45 \mathrm{E}+18$ & 6.5 & 5.9 & 6.7 & 7 & 7 & 1.8 & 1230 & \\
\hline 91 & 3768 & 2010 & 4 & 25 & 205 & -39.28 & 999 & 175.93 & 999 & 60 & 1.4 & 7.9 & 9.4 & 8.4 & $1.48 \mathrm{E}+19$ & $1.76 \mathrm{E}+19$ & 6.7 & 6.2 & 6.9 & 5 & 5 & 5.3 & 3607 & V \\
\hline 92 & 3798 & 2010 & 5 & 25 & 107 & -39.69 & 52 & 177.22 & 53 & 14 & 7.3 & 8.7 & -6.2 & 5.7 & $1.01 \mathrm{E}+18$ & $7.47 \mathrm{E}+17$ & 5.9 & 5.6 & 6.1 & 4 & 4 & 1.8 & 229 & \\
\hline 93 & 3808 & 2010 & 6 & 4 & 24 & -39.87 & 999 & 175.75 & 999 & 41 & 2.4 & 3.7 & 6.4 & 3.4 & $7.13 E+17$ & 4. $48 \mathrm{E}+17$ & 5.8 & 5.6 & 6.0 & 6 & 6 & 1.0 & 250 & \\
\hline 94 & 3816 & 2010 & 6 & 12 & 62 & -38.34 & 999 & 177.73 & 999 & 27 & 0.0 & 0.8 & 2.4 & 0.6 & $1.27 E+18$ & $4.16 \mathrm{E}+17$ & 6.0 & 5.9 & 6.1 & 9 & 9 & 1.4 & 1311 & \\
\hline 95 & 3820 & 2010 & 6 & 16 & 11 & -40.07 & 40 & 176.06 & 57 & 28 & 8.3 & 2.0 & 1.8 & 2.9 & $4.34 \mathrm{E}+18$ & $1.97 \mathrm{E}+18$ & 6.4 & 6.2 & 6.5 & 3 & 3 & 2.4 & 1275 & \\
\hline 96 & 3826 & 2010 & 6 & 22 & 91 & -41.87 & 999 & 174.28 & 999 & 14 & 2.2 & 5.8 & 8.8 & 5.3 & $2.54 E+18$ & $2.73 E+18$ & 6.2 & 5.5 & 6.4 & 2 & 3 & 2.9 & 663 & \\
\hline 97 & 3853 & 2010 & 7 & 19 & 381 & -39.88 & 14 & 175.96 & 18 & 36 & -3.2 & 1.5 & 13.1 & 1.3 & $1.77 E+19$ & $2.39 \mathrm{E}+18$ & 6.8 & 6.7 & 6.8 & 25 & 25 & 4.9 & 3255 & \\
\hline 98 & 3888 & 2010 & 8 & 23 & 375 & -38.71 & 999 & 177.05 & 999 & 38 & 0.8 & 1.7 & -4.9 & 1.4 & $4.55 E+18$ & $1.52 \mathrm{E}+18$ & 6.4 & 6.3 & 6.5 & 22 & 22 & 2.9 & 2256 & \\
\hline 99 & 3932 & 2010 & 10 & 6 & 70 & -39.36 & 20 & 176.52 & 22 & 34 & 0.5 & 3.8 & 14.7 & 2.6 & $4.12 E+18$ & $1.05 \mathrm{E}+18$ & 6.3 & 6.3 & 6.4 & 4 & 4 & 1.9 & 689 & \\
\hline 100 & 3955 & 2010 & 10 & 29 & 91 & -41.16 & 999 & 174.55 & 999 & 31 & -4.1 & 1.7 & -3.2 & 1.0 & $2.83 \mathrm{E}+18$ & $1.34 \mathrm{E}+18$ & 6.2 & 6.1 & 6.4 & 2 & 3 & 1.8 & 1355 & \\
\hline 101 & 4009 & 2010 & 12 & 22 & 30 & -38.6 & 999 & 177.68 & 999 & 22 & -2.3 & 1.0 & 2.2 & 0.9 & $8.52 E+17$ & $3.02 \mathrm{E}+17$ & 5.9 & 5.8 & 6.0 & 4 & 4 & 1.5 & 654 & \\
\hline 102 & 4011 & 2010 & 12 & 24 & 189 & -40.67 & 999 & 175.64 & 999 & 21 & 1.5 & 12.3 & 7.3 & 8.2 & $1.06 \mathrm{E}+18$ & $1.76 \mathrm{E}+18$ & 5.9 & 5.8 & 6.2 & 1 & 3 & 2.1 & 232 & \\
\hline 103 & 4048 & 2011 & 1 & 30 & 60 & -38.87 & 999 & 177.48 & 999 & 21 & -1.4 & 1.7 & 3.0 & 1.7 & $9.19 E+17$ & $4.96 \mathrm{E}+17$ & 5.9 & 5.7 & 6.0 & 5 & 5 & 2.3 & 649 & \\
\hline 104 & 4060 & 2011 & 2 & 11 & 30 & -38.51 & 999 & 177.92 & 999 & 18 & -2.5 & 3.6 & 8.2 & 4.1 & $8.42 E+17$ & 4. $69 \mathrm{E}+17$ & 5.9 & 5.7 & 6.0 & 5 & 5 & 2.2 & 234 & $\mathrm{~N}$ \\
\hline 105 & 4102 & 2011 & 3 & 25 & 145 & -37.88 & 999 & 178.84 & 999 & 9 & 10.5 & 5.4 & 8.2 & 4.1 & $6.79 E+18$ & $3.37 \mathrm{E}+18$ & 6.5 & 6.3 & 6.6 & 7 & 7 & 2.2 & 1255 & \\
\hline 106 & 4148 & 2011 & 5 & 10 & 225 & -41.12 & 999 & 174.13 & 999 & 47 & 6.7 & 7.0 & -13.0 & 2.7 & $8.64 \mathrm{E}+18$ & $2.34 \mathrm{E}+18$ & 6.6 & 6.5 & 6.6 & 5 & 5 & 3.6 & 1421 & \\
\hline 107 & 4180 & 2011 & 6 & 11 & 32 & -40.75 & 999 & 175.94 & 999 & 16 & -8.2 & 4.2 & 14.8 & 8.7 & $1.58 \mathrm{E}+18$ & $1.27 \mathrm{E}+18$ & 6.1 & 5.6 & 6.2 & 2 & 3 & 3.0 & 230 & \\
\hline 108 & 4194 & 2011 & 6 & 25 & 58 & -40.25 & 999 & 177.01 & 999 & 11 & -3.0 & 5.9 & 11.2 & 2.8 & $3.09 E+18$ & $1.08 \mathrm{E}+18$ & 6.3 & 6.1 & 6.4 & 12 & 12 & 2.9 & 632 & \\
\hline 109 & 4199 & 2011 & 6 & 30 & 149 & -39.35 & 999 & 177.87 & 999 & 10 & -1.4 & 1.3 & 7.1 & 1.7 & $5.91 E+18$ & $2.19 \mathrm{E}+18$ & 6.4 & 6.3 & 6.5 & 2 & 3 & 3.2 & 2048 & \\
\hline 110 & 4209 & 2011 & 7 & 10 & 60 & -38.36 & 999 & 177.77 & 999 & 24 & -18.3 & 10.0 & -2.7 & 4.2 & $1.79 E+18$ & $1.03 \mathrm{E}+18$ & 6.1 & 5.9 & 6.2 & 4 & 4 & 2.7 & 238 & \\
\hline 111 & 4234 & 2011 & 8 & 4 & 38 & -39.46 & 999 & 177.31 & 999 & 16 & -4.9 & 2.0 & 7.5 & 2.5 & $2.3 \mathrm{E}+18$ & $7.77 E+17$ & 6.2 & 6.1 & 6.3 & 6 & 6 & 1.6 & 639 & \\
\hline 112 & 4241 & 2011 & 8 & 11 & 86 & -39.07 & 999 & 177.34 & 999 & 21 & 18.9 & 5.4 & -8.8 & 12.2 & $1.93 \mathrm{E}+18$ & $1.39 \mathrm{E}+18$ & 6.1 & 5.8 & 6.3 & 7 & 7 & 3.8 & 232 & \\
\hline 113 & 4250 & 2011 & 8 & 20 & 71 & -38.36 & 999 & 178.87 & 999 & 5 & 12.7 & 5.7 & 17.2 & 3.4 & $1.74 \mathrm{E}+19$ & $5.16 \mathrm{E}+18$ & 6.8 & 6.7 & 6.8 & 10 & 10 & 1.6 & 2033 & \\
\hline 114 & 4312 & 2011 & 10 & 21 & 136 & -40.31 & 999 & 175.8 & 999 & 27 & -6.8 & 3.1 & -0.2 & 2.7 & $6.56 \mathrm{E}+17$ & $3.54 \mathrm{E}+17$ & 5.8 & 5.6 & 5.9 & 6 & 6 & 1.1 & 234 & \\
\hline
\end{tabular}




\begin{tabular}{|c|c|c|c|c|c|c|c|c|c|c|c|c|c|c|c|c|c|c|c|c|c|c|c|c|}
\hline Event & $\begin{array}{l}\text { days } \\
\text { from } \\
2000 \\
\end{array}$ & year & month & day & $\begin{array}{l}\text { duration } \\
\text { (days) }\end{array}$ & lat & $\begin{array}{r}95 \% \mathrm{Cl} \\
(\mathrm{km})\end{array}$ & long & $\begin{array}{c}95 \% \mathrm{Cl} \\
(\mathrm{km})\end{array}$ & $\begin{array}{l}\text { depth } \\
(\mathrm{km})\end{array}$ & $\begin{array}{c}\text { slip(ss) } \\
(\mathrm{cm})\end{array}$ & $95 \% \mathrm{Cl}$ & $\begin{array}{c}\text { slip(ds) } \\
\mathrm{cm}\end{array}$ & $95 \% \mathrm{Cl}$ & $\begin{array}{c}\text { moment } \\
(\mathrm{N} . \mathrm{m})\end{array}$ & $95 \% \mathrm{Cl}$ & $\mathrm{Mw}$ & $95 \% \mathrm{Cl}-$ & $95 \% \mathrm{Cl}+$ & sites & nulls & $\begin{array}{c}\text { rms fit } \\
(\mathrm{mm})\end{array}$ & $\begin{array}{c}\text { fault } \\
\text { area } \\
\left(\mathrm{km}^{\wedge} 2\right) \\
\end{array}$ & $\begin{array}{l}\text { Note: } \\
\text { see } \\
\text { text }\end{array}$ \\
\hline \begin{tabular}{l|l|}
115 \\
\end{tabular} & 4321 & 2011 & 10 & 30 & 21 & -39.12 & 999 & 177.96 & 999 & 10 & -7.0 & 2.0 & 8.6 & 3.6 & $1.03 E+18$ & $4.15 E+17$ & 5.9 & 5.8 & \begin{tabular}{|l|}
6.0 \\
\end{tabular} & 5 & 5 & 2.0 & 228 & \\
\hline 116 & 4343 & 2011 & 11 & 21 & 221 & -37.83 & 999 & 178.75 & 999 & 11 & 12.0 & 6.7 & 10.6 & 6.7 & $4.31 \mathrm{E}+18$ & $2.31 E+18$ & 6.4 & 6.1 & 6.5 & 3 & 3 & 2.4 & 647 & \\
\hline 117 & 4354 & 2011 & 12 & 2 & 64 & -38.64 & 999 & 178.22 & 999 & 11 & -1.4 & 1.9 & 8.2 & 2.1 & $2.17 \mathrm{E}+18$ & $5.39 \mathrm{E}+17$ & 6.2 & 6.1 & 6.2 & 5 & 5 & 3.0 & 637 & \\
\hline 118 & 4358 & 2011 & 12 & 6 & 29 & -38.89 & 999 & 177.97 & 999 & 12 & -7.5 & 2.7 & 12.3 & 4.5 & $1.33 \mathrm{E}+18$ & $5.22 E+17$ & 6.0 & 5.9 & 6.1 & 5 & 5 & 2.3 & 230 & \\
\hline 119 & 4394 & 2012 & 1 & 11 & 84 & -39.35 & 999 & 176.31 & 999 & 41 & -1.2 & 3.1 & 6.7 & 2.8 & $2 \mathrm{E}+18$ & $8.98 E+17$ & 6.1 & 6.0 & 6.2 & 8 & 8 & 1.7 & 709 & \\
\hline 120 & 4404 & 2012 & 1 & 21 & 72 & -38.1 & 999 & 177.53 & 999 & 40 & 11.4 & 2.7 & 4.9 & 3.1 & $6.97 \mathrm{E}+18$ & $2.18 E+18$ & 6.5 & 6.4 & 6.6 & 3 & 3 & 1.1 & 1406 & \\
\hline 121 & 4462 & 2012 & 3 & 19 & 300 & -41.13 & 999 & 174.97 & 999 & 21 & -1.3 & 4.4 & 8.0 & 3.0 & $8.11 \mathrm{E}+17$ & $3.29 E+17$ & 5.9 & 5.7 & 6.0 & 8 & 8 & 2.3 & 236 & \\
\hline 122 & 4556 & 2012 & 6 & 21 & 13 & -38.61 & 999 & 177.89 & 999 & 17 & -0.2 & 2.2 & 4.3 & 1.5 & $4.06 \mathrm{E}+17$ & $2.69 E+17$ & 5.7 & 5.4 & 5.8 & 2 & 3 & 1.0 & 233 & \\
\hline 123 & 4562 & 2012 & 6 & 27 & 112 & -41.11 & 999 & 174.21 & 999 & 44 & 8.1 & 10.9 & -13.7 & 5.6 & $9.55 \mathrm{E}+18$ & $4.86 \mathrm{E}+18$ & 6.6 & 6.4 & 6.7 & 4 & 4 & 5.8 & 1406 & \\
\hline 124 & 4586 & 2012 & 7 & 21 & 15 & -38.46 & 999 & 178.11 & 999 & 15 & -0.2 & 1.2 & 2.4 & 0.6 & $2.33 E+17$ & $1.61 \mathrm{E}+17$ & 5.5 & 5.2 & 5.7 & 1 & 3 & 0.8 & 232 & \\
\hline 125 & 4595 & 2012 & 7 & 30 & 72 & -38.22 & 19 & 178 & 32 & 22 & -4.2 & 1.6 & 4.8 & 1.3 & $1.7 \mathrm{E}+18$ & $4.59 E+17$ & 6.1 & 6.0 & 6.2 & 8 & 8 & 2.1 & 661 & \\
\hline 126 & 4697 & 2012 & 11 & 9 & 86 & -38.05 & 999 & 178.74 & 999 & 8 & -8.7 & 5.6 & 11.4 & 4.7 & $3.76 \mathrm{E}+18$ & $1.48 E+18$ & 6.3 & 6.2 & 6.4 & 4 & 4 & 1.9 & 637 & \\
\hline 127 & 4724 & 2012 & 12 & 6 & 58 & -38.25 & 999 & 178.32 & 999 & 13 & -0.3 & 3.0 & 6.9 & 3.5 & $6.59 \mathrm{E}+17$ & $4.84 \mathrm{E}+17$ & 5.8 & 5.4 & 6.0 & 5 & 5 & 1.9 & 233 & \\
\hline 128 & 4739 & 2012 & 12 & 21 & 16 & -41.21 & 999 & 174.18 & 999 & 41 & -13.1 & 13.8 & 20.8 & 7.0 & $1.47 \mathrm{E}+19$ & $8.08 E+18$ & 6.7 & 6.5 & 6.8 & 1 & 3 & 7.7 & 1398 & \\
\hline 129 & 4743 & 2012 & 12 & 25 & 177 & -39.84 & 999 & 174.63 & 999 & 90 & -10.1 & 9.5 & -12.9 & 4.7 & $1.18 \mathrm{E}+19$ & $7.12 E+18$ & 6.6 & 6.4 & 6.8 & 12 & 12 & 1.5 & 1743 & \\
\hline 130 & 4755 & 2013 & 1 & 6 & 184 & -40.6 & 999 & 174.71 & 999 & 45 & 10.4 & 4.6 & 11.4 & 3.3 & $1.45 \mathrm{E}+19$ & $5.12 E+18$ & 6.7 & 6.6 & 6.8 & 8 & 8 & 3.3 & 2323 & \\
\hline 131 & 4774 & 2013 & 1 & 25 & 94 & -41.45 & 999 & 175.47 & 999 & 8 & -4.1 & 2.0 & -2.9 & 1.5 & $4.66 \mathrm{E}+17$ & $2.31 E+17$ & 5.7 & 5.5 & 5.8 & 4 & 4 & 1.6 & 228 & \\
\hline 132 & 4785 & 2013 & 2 & 5 & 37 & -39.38 & 999 & 177.92 & 999 & 9 & -2.6 & 2.1 & 17.7 & 2.6 & $8.82 \mathrm{E}+18$ & $1.86 \mathrm{E}+18$ & 6.6 & 6.5 & 6.6 & 8 & 8 & 1.8 & 1237 & \\
\hline 133 & 4793 & 2013 & 2 & 13 & 134 & -37.89 & 999 & 178.47 & 999 & 16 & -4.5 & 1.9 & -4.2 & 3.8 & $1.72 \mathrm{E}+18$ & $6.48 E+17$ & 6.1 & 6.0 & 6.2 & 12 & 12 & 2.1 & 659 & \\
\hline 134 & 4799 & 2013 & 2 & 19 & 39 & -40.01 & 18 & 177.26 & 17 & 10 & -15.1 & 5.1 & 27.7 & 4.2 & $8.05 E+18$ & $1.13 E+18$ & 6.5 & 6.5 & 6.6 & 11 & 11 & 2.0 & 632 & \\
\hline 135 & 4807 & 2013 & 2 & 27 & 353 & -40.68 & 999 & 175.01 & 999 & 33 & 2.1 & 4.4 & 27.4 & 3.5 & $2.42 \mathrm{E}+19$ & $4.62 E+18$ & 6.9 & 6.8 & 6.9 & 14 & 14 & 8.4 & 2192 & $\mathrm{~N}$ \\
\hline 136 & 4809 & 2013 & 3 & 1 & 262 & -37.94 & 999 & 177.55 & 999 & 45 & -1.5 & 6.2 & -13.3 & 8.7 & $4.13 E+18$ & $3.26 \mathrm{E}+18$ & 6.3 & 5.9 & 6.5 & 6 & 6 & 3.2 & 731 & \\
\hline 137 & 4826 & 2013 & 3 & 18 & 188 & -39.4 & 999 & 178.05 & 999 & 7 & 1.4 & 4.4 & 14.3 & 1.7 & $7.17 E+18$ & $2.54 E+18$ & 6.5 & 6.4 & 6.6 & 13 & 13 & 1.2 & 1234 & \\
\hline 138 & 4915 & 2013 & 6 & 15 & 244 & -39.66 & 999 & 177.92 & 999 & 7 & -9.5 & 8.0 & -10.1 & 4.9 & $1.74 E+19$ & $1.13 E+19$ & 6.8 & 6.5 & 6.9 & 9 & 9 & 2.4 & 3043 & \\
\hline 139 & 4918 & 2013 & 6 & 18 & 97 & -38.59 & 999 & 178.78 & 999 & 5 & -1.1 & 12.4 & 29.7 & 8.0 & $2.49 E+19$ & $6.23 E+18$ & 6.9 & 6.8 & 6.9 & 10 & 10 & 3.5 & 2031 & \\
\hline 140 & 4935 & 2013 & 7 & 5 & 60 & -41.51 & 11 & 175.1 & 9 & 10 & -6.5 & 1.5 & -5.9 & 1.0 & $2.23 E+18$ & $4.78 E+17$ & 6.2 & 6.1 & 6.2 & 6 & 6 & 2.3 & 639 & \\
\hline 141 & 4949 & 2013 & 7 & 19 & 26 & -41.65 & 18 & 174.35 & 25 & 20 & -14.5 & 5.7 & 42.6 & 9.0 & $1.78 \mathrm{E}+19$ & $4.44 E+18$ & 6.8 & 6.7 & 6.8 & 6 & 6 & 9.1 & 979 & E \\
\hline 142 & 4962 & 2013 & 8 & 1 & 197 & -40.37 & 999 & 175.35 & 999 & 34 & 1.9 & 4.2 & 8.7 & 5.6 & $2.6 \mathrm{E}+18$ & $1.65 E+18$ & 6.2 & 5.9 & 6.4 & 7 & 7 & 3.1 & 675 & \\
\hline 143 & 4977 & 2013 & 8 & 16 & 174 & -42.01 & 999 & 174.21 & 999 & 16 & 3.1 & 21.4 & -63.1 & 20.7 & $1.54 E+20$ & $1.23 E+20$ & 7.4 & 6.9 & 7.6 & 1 & 3 & 61.3 & 5934 & E \\
\hline 144 & 4977 & 2013 & 8 & 16 & 32 & -41.61 & 999 & 174.43 & 999 & 19 & -16.7 & 3.8 & 26.9 & 7.7 & $1.67 \mathrm{E}+19$ & $5.03 E+18$ & 6.7 & 6.7 & 6.8 & 9 & 9 & 7.7 & 1324 & E \\
\hline 145 & 4999 & 2013 & 9 & 7 & 160 & -40.98 & 999 & 174.36 & 999 & 43 & 25.7 & 6.5 & 11.4 & 5.3 & $1.59 E+19$ & $4.03 E+18$ & 6.7 & 6.7 & 6.8 & 9 & 9 & 5.5 & 1402 & \\
\hline 146 & 5012 & 2013 & 9 & 20 & 32 & -39.02 & 999 & 177.91 & 999 & 12 & -1.5 & 1.7 & 6.8 & 1.7 & $1.78 \mathrm{E}+18$ & $5.81 E+17$ & 6.1 & 6.0 & 6.2 & 6 & 6 & 3.4 & 636 & \\
\hline 147 & 5013 & 2013 & 9 & 21 & 136 & -39.32 & 999 & 175.81 & 999 & 62 & -6.2 & 4.2 & 3.9 & 2.3 & $4.62 \mathrm{E}+18$ & $1.55 E+18$ & 6.4 & 6.3 & 6.5 & 10 & 10 & 2.6 & 1476 & V \\
\hline 148 & 5015 & 2013 & 9 & 23 & 100 & -38.49 & 999 & 178.89 & 999 & 4 & -4.8 & 12.1 & -14.0 & 7.3 & $8.2 E+18$ & $4.86 E+18$ & 6.5 & 6.3 & 6.7 & 5 & 5 & 1.0 & 1227 & \\
\hline 149 & 5052 & 2013 & 10 & 30 & 61 & -37.97 & 999 & 178.97 & 999 & 6 & 10.7 & 12.2 & 13.0 & 7.4 & $8.87 \mathrm{E}+18$ & $7.87 \mathrm{E}+18$ & 6.6 & 5.9 & 6.8 & 2 & 3 & 2.1 & 1240 & \\
\hline 150 & 5091 & 2013 & 12 & 8 & 61 & -39.02 & 999 & 177.99 & 999 & 10 & 1.1 & 1.6 & -3.0 & 1.3 & $3.04 \mathrm{E}+17$ & $1.77 \mathrm{E}+17$ & 5.6 & 5.3 & 5.7 & 3 & 3 & 1.1 & 229 & \\
\hline
\end{tabular}




\subsection{Effects of layering}

Section 2.6.4 introduced the logic behind the decision to further investigate the effects of layering. This was not part of the original scope of work, but appeared to become a subject that 'could not be ignored'. Nevertheless, because it was not part of the initial project, and the magnitude of the possible effects were not initially well understood and could not therefore be planned for, it became clear that this part of the investigation would need to form a separate chapter which may serve as a pointer to possible future work. During development of this investigation it became clearer that it might be possible to use this work to propose a $1^{\text {st }}$ order approximation that could be applied to results obtained using the more conventional homogenous half-space inversion.

\subsection{Methods}

It was decided to adopt code published by $R$ Wang et al. [2003]. The code package EDGRN/EDCMP was downloaded, and compiled using Simply Fortran v 2.14 (which uses the OpenSource code GNU Fortran), after checking that the array sizes declared in the files edgglobal.h and edcglobal.h were large enough for this application. The code is in two parts - EDGRN generates the Green's functions for the layer model, and EDCMP calculates displacements (and if required, strains, stresses and tilts) using either the layer code, or an implementation of Okada's code for the half-space model.

Initially the code was used to examine the possible effects of layering on shallow lowdip thrust faults such as may be encountered along the NE coast of the North Island, since any effects due to layering here were considered more likely to be more pronounced than for the deeper SSEs located in the SW of the lower North Island. Exploration borehole data was obtained from Ministry of Economic Development database (https://data.nzpam.govt.nz/GOLD/ststem/mainframe.asp) in order to supplement data on shallow strata. These initial investigations were sufficient to suggest that the magnitude of possible effects on surface displacements could be several tens of percent, with significant implications for any source displacements or moments estimated by inversion. This therefore prompted a more systematic approach. 


\subsection{Code validation}

Since the EDCMP package uses two separate code sections for layered and homogenous models, both had to be validated.

In order to validate the package on the homogenous half-space model, the same fault model was used as had been previously used to validate the Matlab code, i.e. from Cattin et al. [1999]. The results were considered totally consistent with those from Okada, as may be seen in Appendix 7.

The layering code was validated by comparison with Fig. 5 in Cattin et al. [1999]. Finally, consistency between the layered and homogenous half-space parts of the code was confirmed by modeling a layered scenario with trivial differences in elastic properties. Details are shown in Appendix 7.

\subsection{Estimating subsurface profiles}

It is not trivial to obtain data on which to base a layered subsurface model. The 3D velocity model of Eberhart-Phillips et al. [2010] provides a good starting point, but, since there are few earthquakes in the top $10 \mathrm{~km}$ of strata, is not well resolved in the shallower horizons that are of interest in this project.

Oil and gas exploration wells generally penetrate only to less than $5 \mathrm{~km}$. Seismic reflection studies, whilst giving good coverage offshore in the possible oil or gas exploration basins, provide very much less coverage onshore. The available seismic refraction and receiver function studies are also generally not well resolved in the upper horizons.

Compilations of geological studies, exploration well logs and seismic data have all been used in the updates of the QMAP 1:250,000 Geological Maps of New Zealand series recently completed by GNS Science. These provide a number of cross-sections that give age-related stratigraphies covering much of the country. However, there remains missing the final link between stratigraphy and geophysical properties, or, more specifically, indicators of elastic modulus or seismic velocities. In this respect, Stagpole [1997] provides some velocity-depth correlations for a range of stratigraphic ages from 
both the North Taranaki and West Coast exploration wells, as well as the East Coast wells.

As part of this review, a number of East Coast exploration wells logs were examined and compared with velocity profiles extrapolated for the well locations from EberhartPhillips et al. [2010]. This is illustrated in Fig. 4.3a, which shows the stratigraphic successions at 7 wells, and $V_{P}$ profiles at midpoints of the Eberhart-Phillips et al. horizons for these locations. The 7 well locations can be identified in Fig. 4.3b.

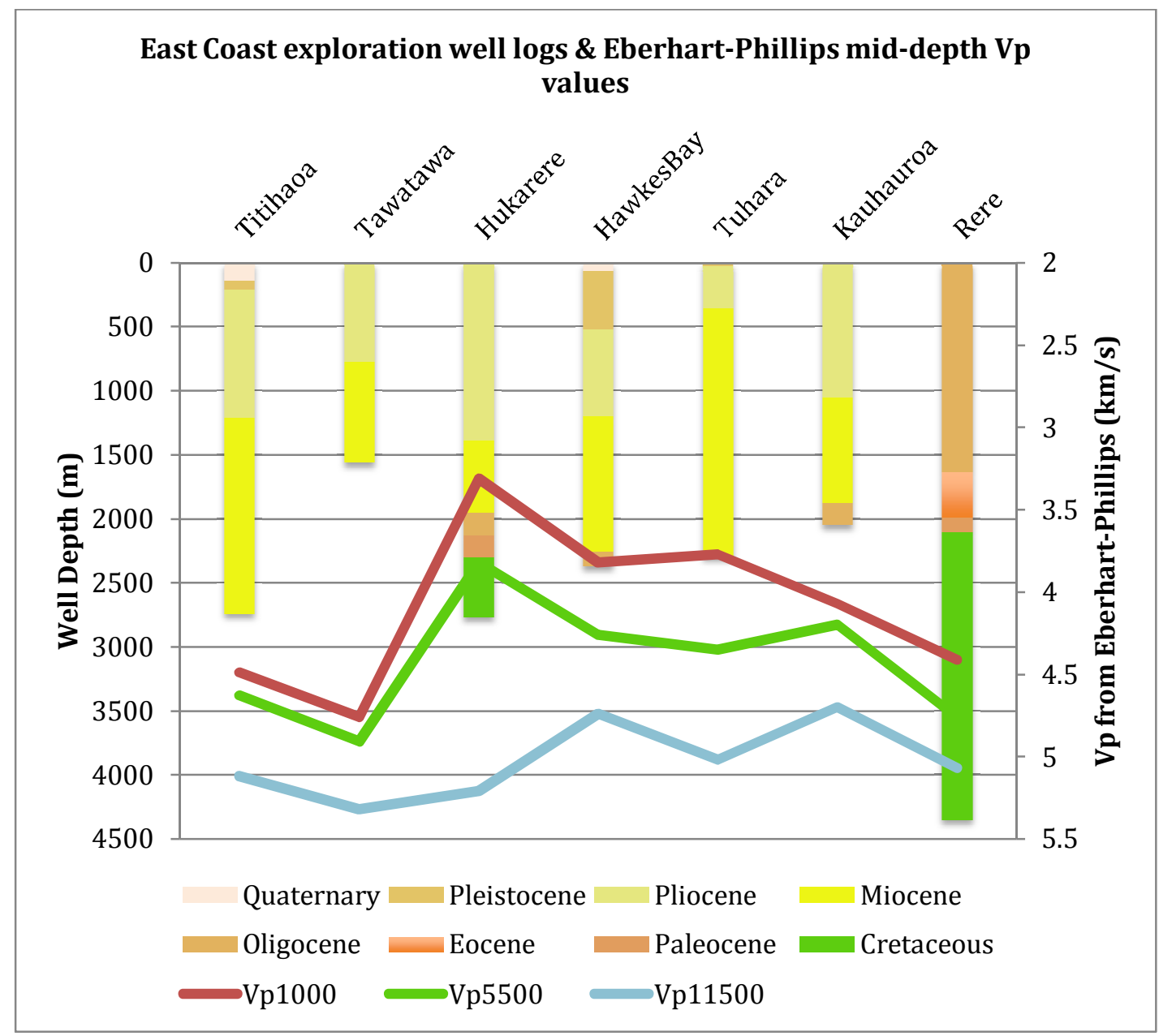

Figure 4.3a: Correlation between exploration well log stratigraphy and seismic velocity. Stratigraphy is displayed conventionally as a function of depth on the left ordinate axis. $V_{P}$ is plotted for 3 depths $(1000 \mathrm{~m}, 5500 \mathrm{~m}$ and $11500 \mathrm{~m})$ as heavy lines with the scale on the right ordinate axis. These depths are selected as mid-points of horizons extrapolated from Eberhart-Phillips et al. [2010] for selected East Coast locations. For example, taking the heavy brown line, this indicates that $\mathrm{V}_{\mathrm{p}}$ at $1000 \mathrm{~m}$ depth is indicated to be approximately $3.3 \mathrm{~km} / \mathrm{s}$ at Hukarere, and approximately $4.4 \mathrm{~km} / \mathrm{s}$ at Rere. The stratigraphy at $1000 \mathrm{~m}$ depth shows Pliocene in the Hukarere well, and Oligocene at Rere, hence indicating some degree of correlation, since velocity is expected to increase with age. Wells are arranged in order (left to right) from south to north, corresponding, approximately, to nearby cGPS sites BIRF (Titahaoa) to RAWI (Rere). 
It can be observed that whilst there appears some correspondence between stratigraphy and velocities, the correlation is relatively poor, possibly reflecting the poor resolution of velocities at shallow depths.

Another source of data is the Basin maps produced by GNS Science as part of the Petroleum Exploration database. These show depth contours for each of the major oil/gas or potential oil/gas basins around the country. The ones of relevance to this study are Taranaki, Whanganui, Raukumara, East Coast and the northern part of the Canterbury basin. An example for the East Coast basin is shown in Fig, 4.3b.

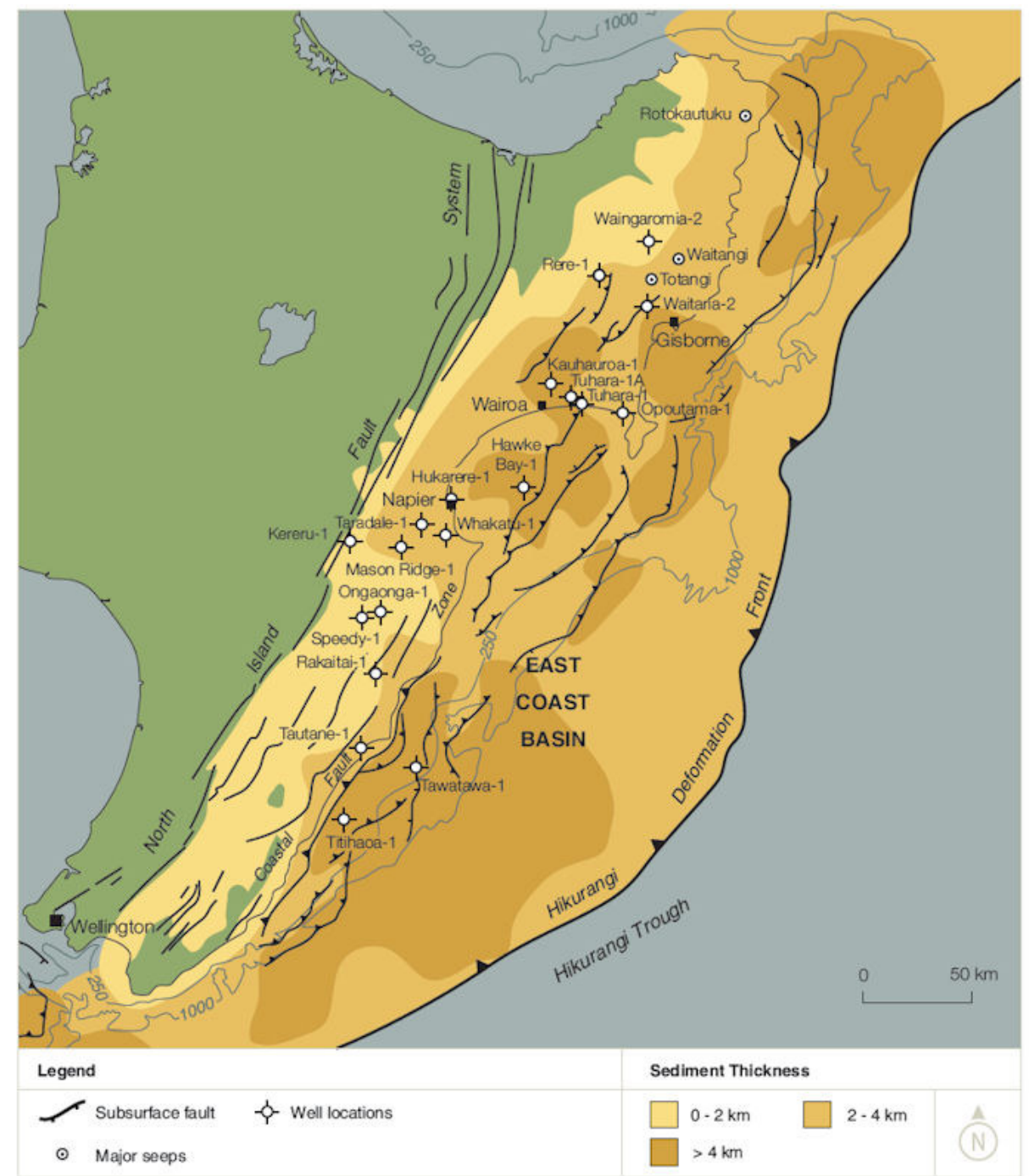

Figure 4.3b (reproduced from GNS Petroleum Exploration Database): Example of sedimentary basin map. In these maps, rocks older than Jurassic - Early Cretaceous are considered to be economic basement. Basin limits have been drawn at the known or inferred position of the 1000 metre sediment thickness isopach. 


\subsection{Tests on models of the Hikurangi subduction interface}

In order to obtain some idea of the possible effects of layering on surface displacements for events in the NE and SW of the Hikurangi subduction zone, two basic models were explored. In both cases, the model fault was assumed to be $30 \mathrm{~km}$ long, $20 \mathrm{~km}$ wide (down dip) and the source displacement was in all cases $1 \mathrm{~m}$. In each case, the layered model displacements were compared with those resulting from use of an Okada model with equivalent half-space elastic parameters calculated on a depth interval-weighted basis. For the layered model, the Greens function calculations in EDGRN use input parameters based on seismic velocities and densities, and hence the depth-weighting calculation used the square of the velocities as a proxy for elastic modulus - although it should be noted that the Okada model is relatively insensitive to changes in elastic properties.

In the case of the NE set of models, the slip was modeled at rake $270^{\circ}$ (i.e. thrust), and for the $\mathrm{SW}$ set, $000^{\circ}$ (strike-slip). In both cases strike direction was maintained at $000^{\circ}$, which corresponded with the $\mathrm{X}$ coordinate in the Okada formulation. The Y coordinate (across strike) is therefore in the east direction for the NE models, and south direction for the SW models. The Z coordinate is downwards in the EDCMP code, but has been inverted to correspond to upwards in all the plots.

In the NE case, the model assumed a dip of $-10^{\circ}$ and a depth of $21 \mathrm{~km}$. Fig. 4.4a illustrates the results for one example, where the shallow profile was extrapolated from the $\log$ of exploration well Tuhara-1, and the deeper profile (beyond $2 \mathrm{~km}$ ) from Eberhart-Phillips et al. [2010]. 


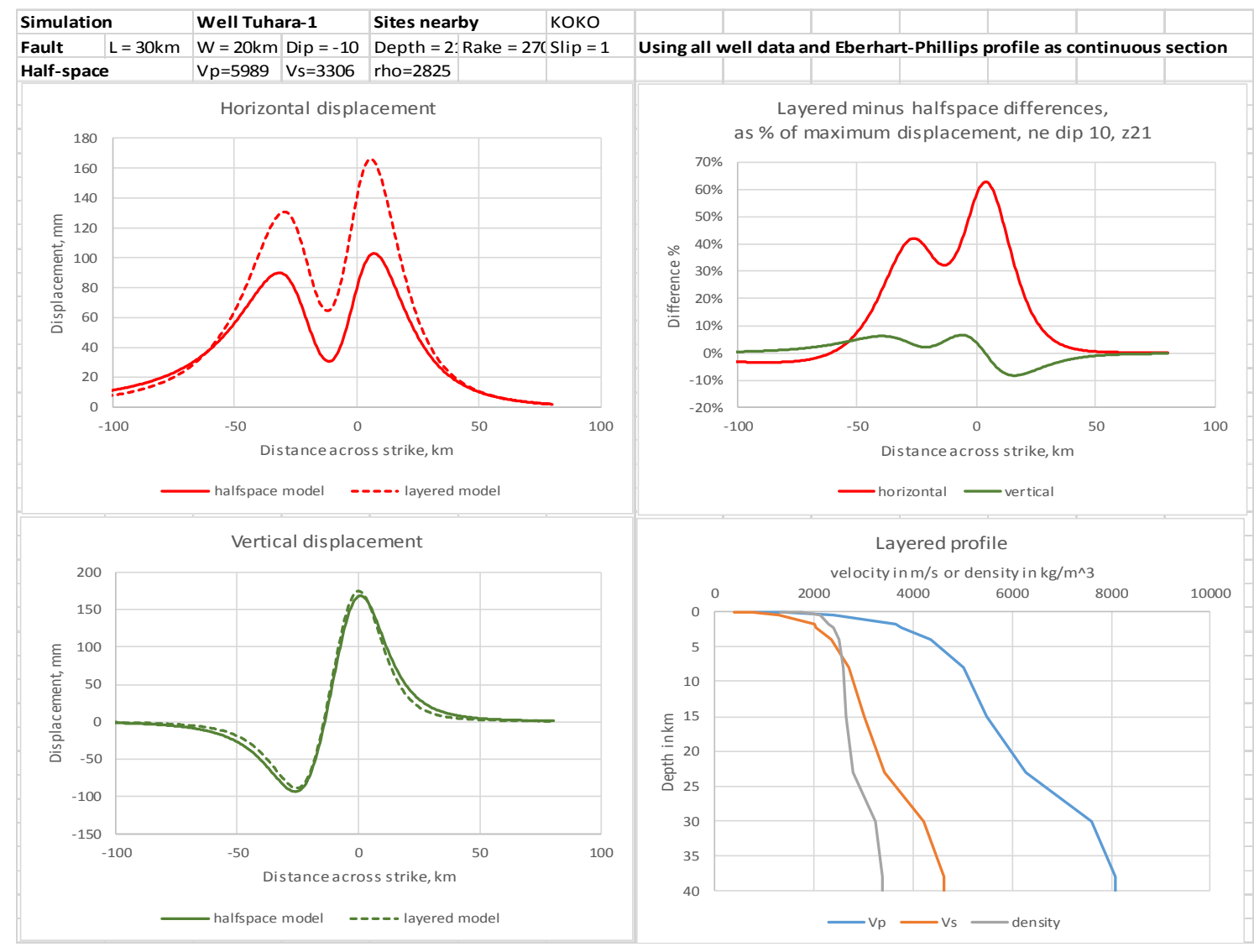

Figure 4.4a: Comparison of modeled surface displacements arising from EDGRN/EDCMP layered model and the equivalent Okada half-space model for a simulated shallow-dipping thrust fault at $21 \mathrm{~km}$ depth along the NE coast of North Island. From top left clockwise, the plots show: surface displacements from the 2 models in the east direction across the surface projection of the strike at the centre of the length of the fault; percentage change in surface displacement in the layered model as a proportion of the maximum in the Okada model; the profile of $\mathrm{V}_{\mathrm{P}}, \mathrm{V}_{\mathrm{S}}$, and density with depth used in the layered model; and the surface displacements from the 2 models in the upwards direction across the strike of the fault.

For the second set of comparisons in the SW of North Island, there were no suitable borehole data available, and the elastic parameters were therefore taken by extrapolation from the SAHKE profile [Henrys et al., 2013]. Fig. 4.4b shows one example comparison for this scenario, with the fault modeled at $27 \mathrm{~km}$ with a dip of $-22^{\circ}$.

In both cases, the models appeared to suggest significant differences in maximum surface displacements, particularly in the horizontal direction, between the simple homogenous half-space model of Okada, and the layered calculations in EDGRN/EDCMP. For the shallower NE models, the differences were greater than for the SW models, but in both cases, it seemed that the effects of layering should not be ignored. 


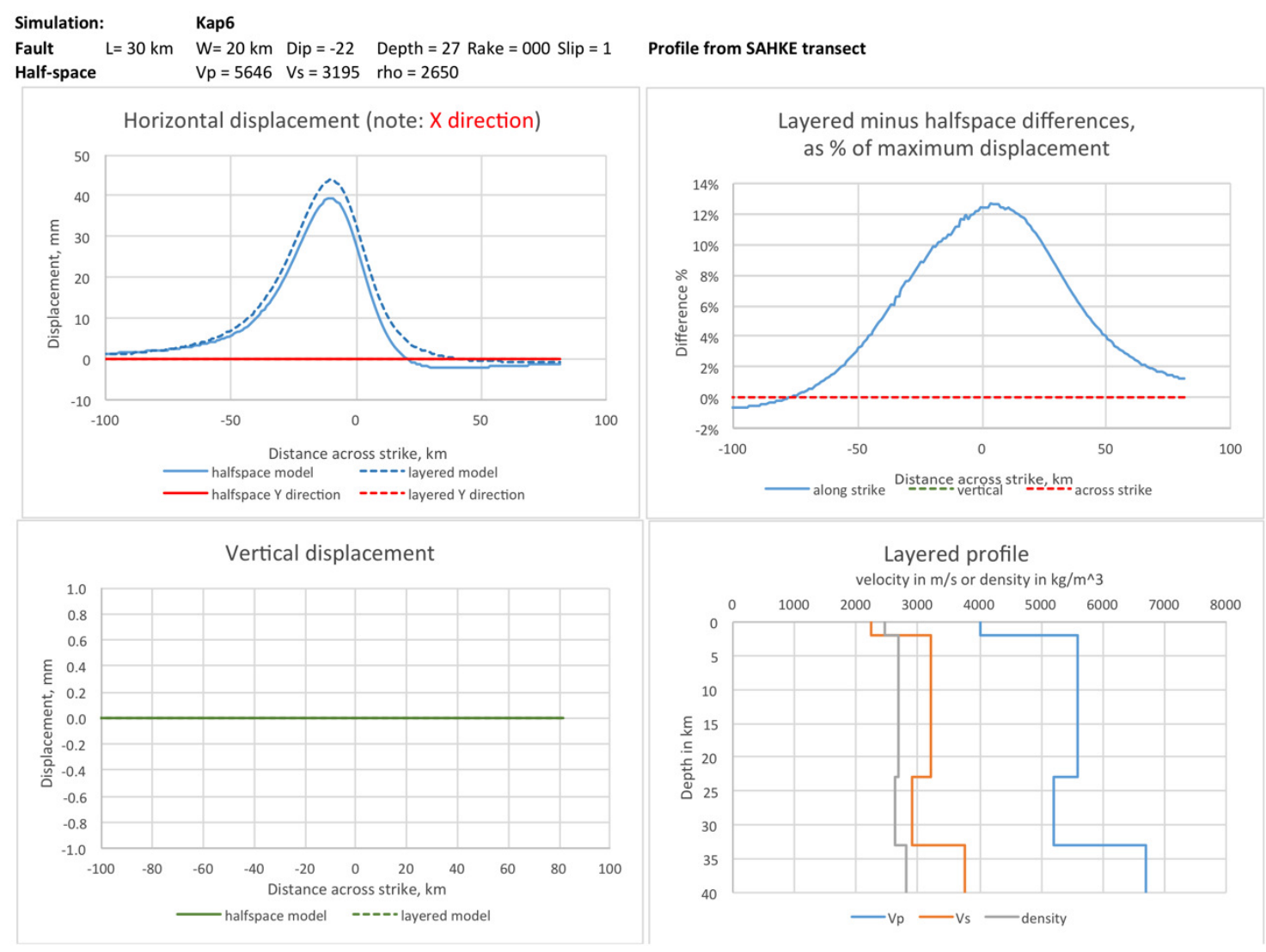

Figure 4.4b: Comparison of modeled surface displacements arising from EDGRN/EDCMP layered model and equivalent Okada half-space model for a simulated strike-slip fault at $27 \mathrm{~km}$ depth and dipping $22^{\circ}$ along the SW coast of North Island. Plots have the same meaning as in Fig. 4.4a, except that the X direction (along strike) is now effectively eastwards. The depth profile is taken from the SAKHE transect.

\subsection{Systematic parametric study of the effects of layering}

Preliminary investigations of the effects of layering on surface displacements had shown that whilst the Okada solution was relatively insensitive to changes in the elastic properties of the half-space, this was not the case in the layered models. It also became clear that the properties and depth profiles of relatively shallow strata above the subduction interface would have a significant effect on the modeled surface deformations. Furthermore, excepting some specific locations where exploration well or seismic data were available, the production of detailed layered models for every cGPS site would be difficult. Whilst ideally, producing an array of Green's Functions for every site would provide the basis for extending a layered model across the country, there was insufficient data of adequate quality to do this. 
It was therefore decided to investigate whether a simple model using a single softer layer plus a half-space could be used as a first order approximation. In order to assess this proposal, a parametric study was carried out, in which models were constructed with all parameters held constant except the one being investigated. Relevant parameters were considered to be: fault dip and source rake, fault depth, upper layer thickness and modulus. It was known that surface displacement response to changes in fault area and source displacement were linear, so these parameters were kept constant throughout. The details of the study are shown in Appendix 13.

\subsection{Proposal for $1^{\text {st }}$ order correction to the Okada model}

Following on from the analysis detailed in Appendix 13, it is suggested that a simple linear correction could be applied to the Okada solutions, if the subsurface at each cGPS site can be simplified into 2 layers, with elastic properties for the upper layer and the lower half-space being considered similar across the region, but with the thickness of the upper layer being the sole variable. In that case, a simple set of linear equations can be applied for each of the three components:

$$
\begin{aligned}
& \text { Ux Layer }=(0.0590 * Z+0.763) * \text { Ux Okada }(r m s \text { error }=0.8 \%) \ldots . . . \text { Eq. }(4.6 a) \\
& \text { Uy Layer }=(0.0203 * Z+1.131) * \text { Uy Okada }(r m s \text { error }=1.4 \%) \ldots . . . \text { Eq. }(4.6 b) \\
& \text { Uz Layer }=(0.0070 * Z+1.019) * \text { Uz Okada }(\text { rms error }=0.7 \%) \ldots \ldots . \text { Eq. }(4.6 c)
\end{aligned}
$$

Where $\mathrm{Z}$ is the thickness of the upper layer in $\mathrm{km}$., and rms error refers to the non-linearity associated with the linear approximation.

Since the value of Ux Okada $=0$ on the centre-line of a thrust fault, the first equation becomes irrelevant in that case, and similarly for the strike-slip case, only the Ux correction becomes of any real relevance.

Values of upper layer thickness for each GPS site have been estimated to the nearest km from either:

- interpolation as depth to basement from sections shown in the GNS Geological maps 1:250,000 series, or 
- sedimentary basin depths mapped by GNS in the Petroleum Basin Exploration database $[G N S]$

Wherever possible, precedence has been given to the first source. In both cases basement has been taken as the interface between Cenozoic to Cretaceous sediments (or volcanoclastic and pyroclastic deposits in the TVZ), and rocks shown as Jurassic to early Cretaceous. Fig. 4.6 illustrates the variation in upper layer thickness between the cGPS sites used in this project.

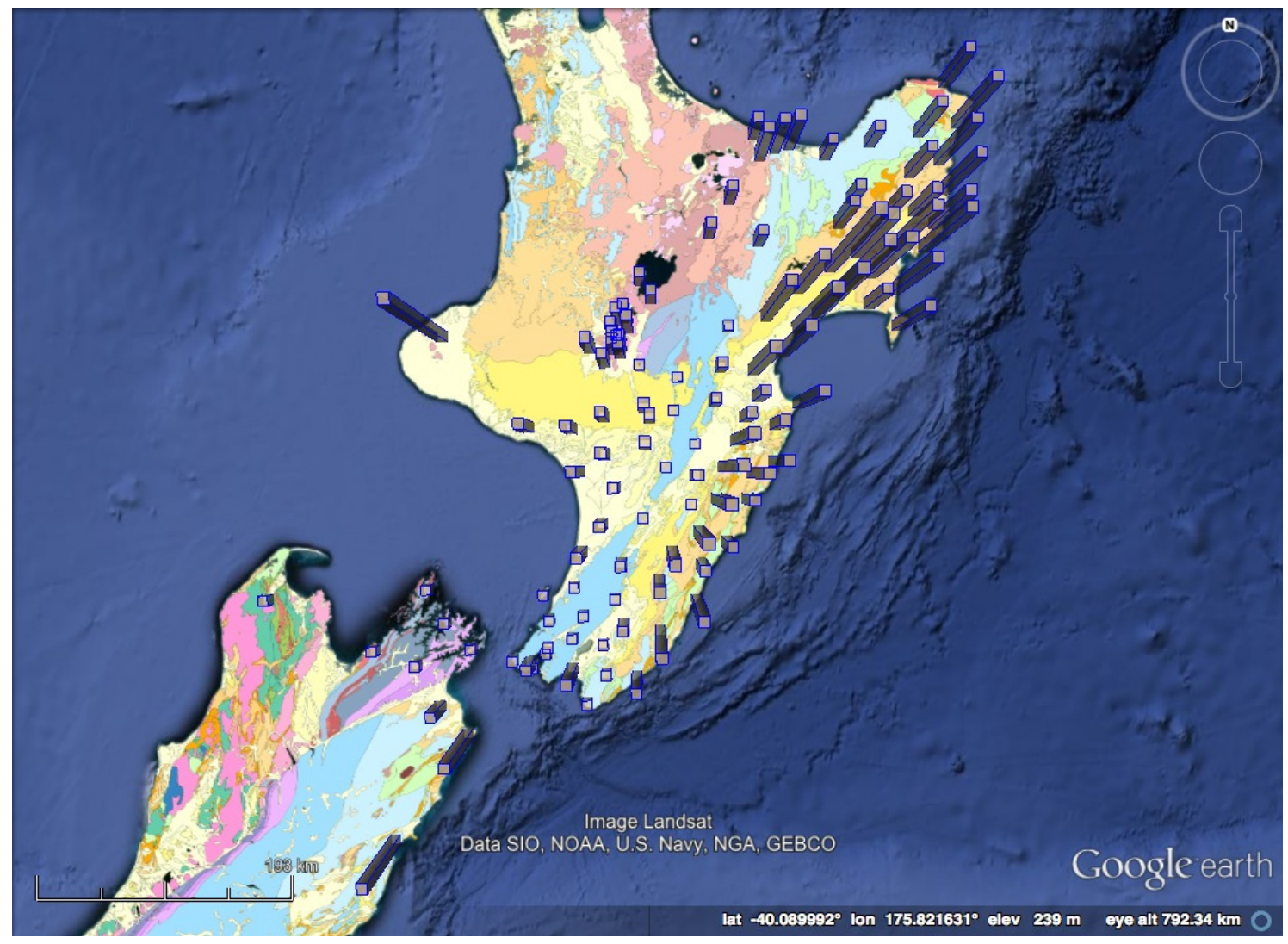

Figure 4.6: Representation of variation in estimated thickness of upper layer (see text) at each cGPS site used in this project, superimposed over GoogleEarth image with overlay of surface geology courtesy GNS Science (for legend, see Appendix 1). Scaling: bar height at New Plymouth represents a thickness of $6 \mathrm{~km}$.

In practice, inversions would be carried out using the Okada model, and the predicted motions of unit displacements on the faultlets would be modified by the equations. Within the time constraints of the project it has not been possible to test this proposal. 



\subsection{Discussion}

\subsection{Residual GPS noise}

Fig. 3.1.1d illustrates that the noise reduction codes have been very successful in removing coloured (correlated) noise, and in reducing overall noise spectral power by over $10 \mathrm{db} / \mathrm{rad} / \mathrm{sample}$. The remaining noise is primarily random in character, with levels in the region of 1 to $2 \mathrm{~mm}$ (rms error residual) in $\mathrm{E}$ component.

With respect to the issue of some sites being noisier than others, Fig. 3.2.1b shows that, with the exception of sites around Mount Ruapehu, the noisier cGPS sites tend to be located on the eastern edges (leeward) of mountainous areas where turbulence and cloud cover may be a dominant factor. The Civil Aviation Authority [CAANZ, 2006] indicates that:

"When the wind becomes strong, moderate to severe turbulence will be encountered on the lee side, from the surface to well above the mountain tops. In the Southern Alps quite severe lee turbulence can be experienced several miles downwind of some peaks and considerably above their height. Most mountain waves in New Zealand result from prevailing westerly winds ... A rotor is a large closed eddy that forms in the lee of a mountain range, or any obstacle in the airflow, and is an area of severe turbulence. Rotors are usually found under the crests of mountain waves, often within 3000 feet vertically of the generating ridge"

This is pictorially illustrated in Fig 5.1, taken from the NZCAA publication.

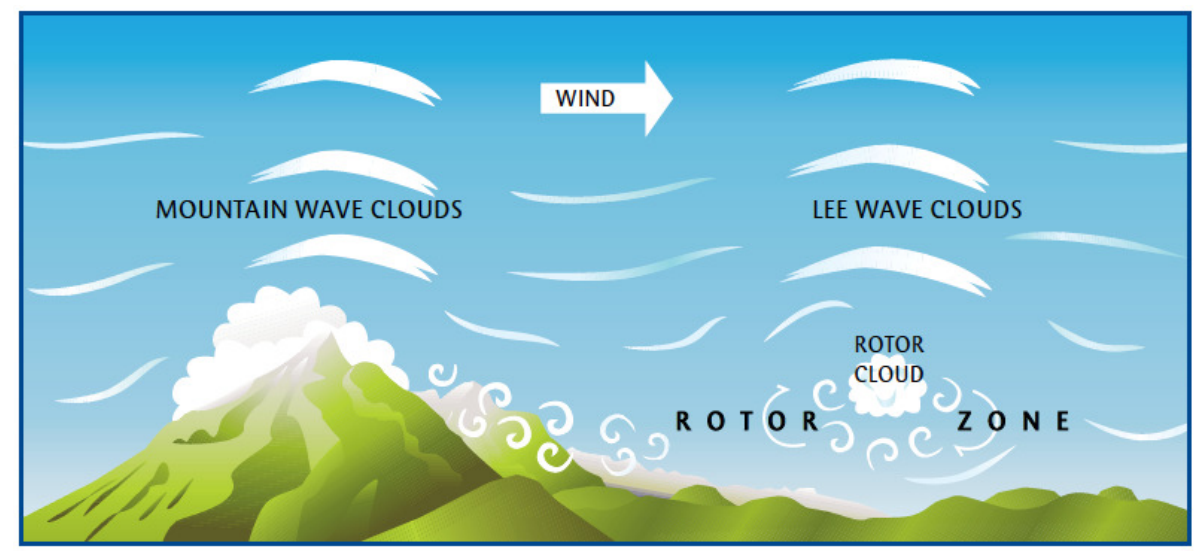

Figure 5.1 (from CAANZ [2006]): Illustrating turbulence effects that occur in the lee of mountain ranges under prevailing wind conditions. 
It will be noted that tropospheric weather effects, and especially water vapour variations and possibly scattering effects of rain/hail/snow, on cGPS measurements cannot easily be corrected [Gregorius and Blewitt, 1998] and must therefore have a direct effect on noise levels. This would seem a probable, but therefore uncontrollable, cause of the most of the higher residual noise levels observed at some sites.

\subsection{Surface displacements}

\subsubsection{Secular velocities}

The overall secular velocities determined in this work are consistent with those published by Lamb and Smith [2013], although it must be noted that there is a small bias caused by the different frames of reference used. Nevertheless, for cGPS sites that had been in operation for more than a couple of years when Lamb \& Smith carried out their analysis, the standard deviation of differences between the values published in their Table 1 and those shown in Appendix 11 of this work are relatively low: $+/-2.0$, 1.2 and $1.8 \mathrm{~mm}$ respectively for the $\mathrm{E}, \mathrm{N}$ and $\mathrm{U}$ components. Some sites, that had been in operation (in Lamb \& Smith's work) for less than $30 \%$ of the time that they had been in operation for this work, showed significant differences in secular velocities illustrating the need to be cautious in studies of this nature when using only 12 to 18 months of cGPS data (especially in time series that include transients).

Fig. 3.3a and Appendix 11 show that, particularly along the NE coastal areas from Hawkes Bay through to the tip of the Raukumara Peninsula, secular horizontal velocities are strongly affected by SSEs. The principal secular surface motion due to SSEs in this region is almost eastwards, or updip on the subduction interface. The overall pattern of surface movement (i.e. ignoring SSEs - red vectors in Fig. 3.3a) shows a clockwise rotation, similar to that shown by other authors for campaign GPS results.

Most of the literature to date concerning the secular velocity field in the North Island has covered the results of analyses on either campaign GPS velocities, or overall average secular velocities from cGPS installations. Lamb and Smith [2013] have 
summarized much of the relevant literature. A recent re-analysis of plate motions and global strain rates from 18,356 GPS locations, in which transients and SSEs have been specifically excluded, shows an apparently fine spatial resolution $\left(0.2^{\circ}\right.$ latitude and $0.25^{\circ}$ longitude) [Kreemer et al., 2014], but there has been significant smoothing to fit the global model, so that details on the scale that we are concerned with are not welldefined. Wallace and Beavan [2010] discussed the SSE-free velocity field (or "interSSE velocities" in their nomenclature), in terms of the coupling coefficient, but did not identify any rotational inhomogeneity.

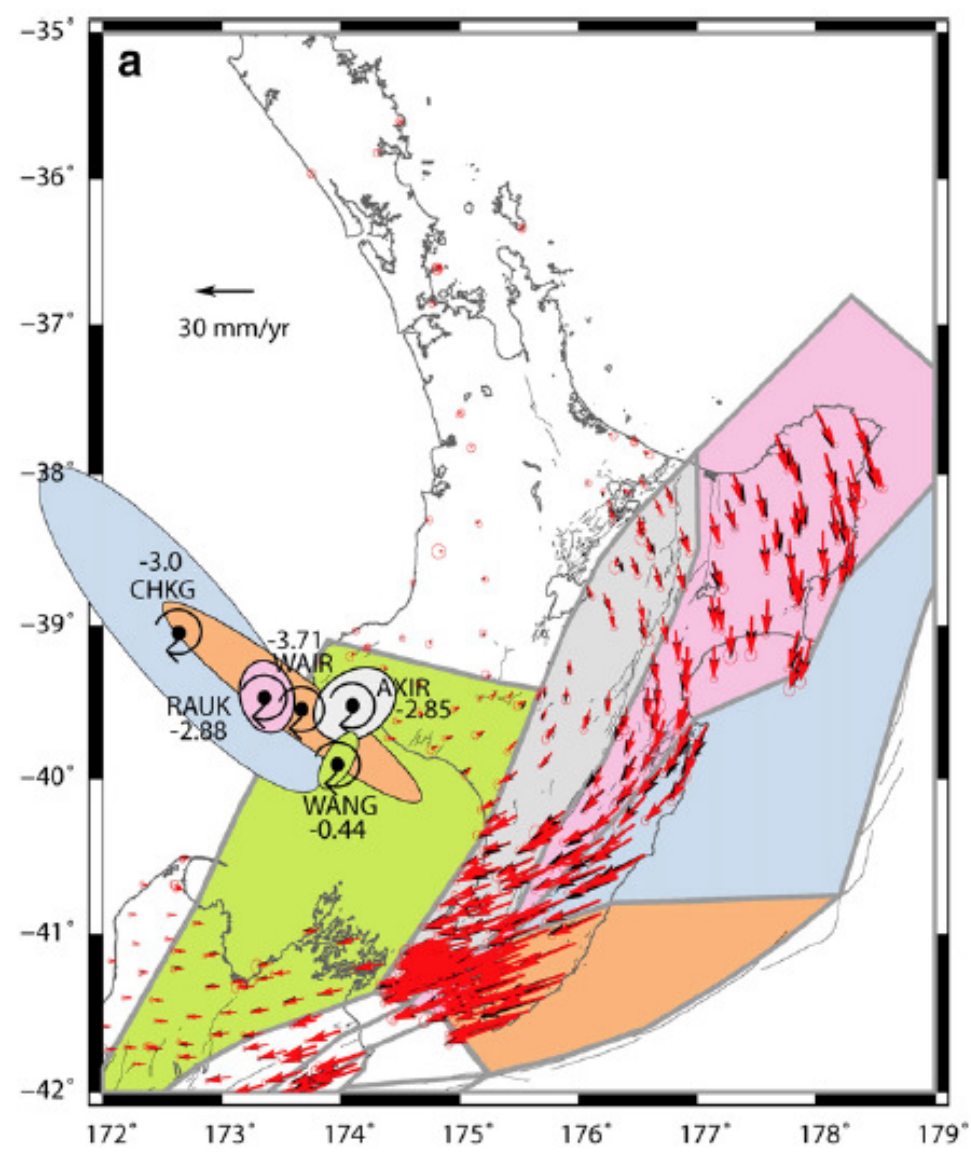

Figure 5.2.1a (reproduced from Fig. 2a of Nicol and Wallace [2007]): GPS velocity field (red arrows) and velocities predicted from block model (black arrows) in the North Island and northwestern South Island resulting from over a decade of GPS campaign measurements. All velocities are shown relative to the Australian Plate. Error ellipses are at $68 \%$ confidence level. Locations and uncertainty ellipses of poles of rotation for each block (estimated in the inversion) relative to the Australian plate are shown, with rotation rates in ${ }^{\circ} \mathrm{Ma}$ (negative numbers indicate clockwise rotation). The block name that each pole corresponds to is denoted by the four-letter code next to the pole (WAIR = Wairarapa block; AXIR = Axial Ranges block; RAUK= Raukumara block; $\mathrm{CHKG}=$ Central Hikurangi block; WANG=Wanganui block). 
Nicol and Wallace [2007] compared campaign GPS data with long-term geological data for the Hikurangi subduction margin and concluded that they were compatible within fairly broad limits of precision. They divided the margin into a number of tectonic blocks and used the campaign GPS data to determine poles of rotation. These are shown in Fig. 5.2.1a, reproduced from Fig. 2a of their paper.

It is noted that the rotation poles shown in Fig. 5.2.1a are located to the south or west of Taranaki, an area somewhat to the southwest of the overall average pole estimate from this work.

If the secular component due to the average of SSEs is removed, a different picture emerges for the SSE-free component, or underlying short-term strain (blue vectors in Fig. 3.3a), which, whilst still indicating some degree of overall clockwise rotation, would suggest an overall pole located much further north (closer to Auckland, see Appendix 13, last page). A pole situated in the North is more consistent with the indications from integration of Holocene fault slip rates [Beanland and Haines, 1988], and paleomagnetism [Lamb, 1988], as shown in Fig. 14b of Lamb [2011]. Moreover, the underlying SSE-free velocity field appears at first sight to be more consistent in direction over much of the eastern North Island, which could suggest a smaller rotational component due to 'behind-arc spreading' than discussed by some authors [Beavan et al., 2002; Lamb and Smith, 2013; Wallace et al., 2004; Wallace et al., $2012 b]$.

Dividing the overall area into smaller regions in which the strain fields might be considered relatively homogenous allows observations to be made on how the principal strains and rotation components may vary over the North Island. However, the results should be considered circumspectly, since small changes in the number of sites selected in each region, or the choice of individual site to be taken as reference, can have significant effects on the magnitudes of the strain and rotation rates. In this respect, the uncertainties shown in the table in Appendix 12 relate only to the specific geometries analysed, and significantly under-estimate the overall uncertainties that would result from changing the regional boundaries. Nevertheless, the azimuths of the principal strain components, as shown in Figs. $3.3 \mathrm{~d}$ and $3.3 \mathrm{e}$, seem to remain reasonably consistent. 
The SSE-free principal strain components shown in Fig. 3.3d are consistent with geological evidence for the Wellington and TVZ regions - where the average SSE strains are relatively small [Beanland and Haines, 1988; Darby and Beavan, 2001; Seebeck, 2012], and results from campaign GPS work [Beavan and Haines, 2001]. Our results for the East Coast appear variable, but seem, nevertheless, consistent with indications by Dimitrova et al. [2014]. After removing the effects of locking, these authors indicate extension in Raukumara and southern Hawkes Bay, and contraction in Gisborne and Wellington. Extension in eastern Raukumara is supported by evidence from earthquake focal mechanisms [Reyners and McGinty, 1999] and from geology [Berryman et al., 2009], and uplift and extension in the peninsula has been modeled by Upton et al. [2003]. Geological evidence also supports the hypothesis of extension south of Hawkes Bay [Cashman and Kelsey, 1990; Cashman et al., 1992].

However, there is evidence of both spatial and temporal variation in principal strain directions in the Raukumara Peninsula [Árnadóttir et al., 1999; McCaffrey, 2014; Thornley, 1996]. Thornley [1996], for example, concluded that:

"Strain for the period 1937 - 1986 around Poverty Bay reveals spatial variation in strain on a scale of $10-20 \mathrm{~km} . "$ (p.184)

and

"Around Poverty Bay, the axis of relative extension trended north-east between 1875 and 1937, whereas for the period 1937 - 1986 the extension axis trended south-east. A similar, but later, change took place in the Tolaga Bay area, where south-easterly relative extension for 1875 - 1925 and 1925 - 1974 changed to north-south relative extension for the period 1974 - 1995." (p. 184)

McCaffrey [2014] suggested that principal strain directions in this region should be considered ephemeral and indicative of changes in locking over time, as compared with Walcott [1984] and Nicol and Wallace [2007] who suggested that geological, geodetic, GPS and paleomagnetic evidence were reasonably consistent and suggestive of stability over the last 1.5 Ma. Apparently differing views may simply reflect the differing time scales being considered. Lamb and Smith [2013] have explained how the available span of observations where SSEs occur can affect the perception of locking.

Nicol and Wallace [2007] noted that: 
"The GPS velocity field (Fig. $2 a$ [Fig. 5.2.1a above]) indicates no differential rotation between Raukumara and Wairoa domains (i.e. the entire eastern North Island is rotating at a rate of $3-4 \% \mathrm{Ma}$ ), and we propose that the boundary between the rotational domains is a relict feature that is no longer active." (p.406)

The reference to the Raukumara and Wairoa domains is relevant, in that paleomagnetic evidence, whilst apparently giving rise to different interpretations [Nicol et al., 2007], does indicate a degree of discontinuity in historical rotational terms between these two domains, with the latter having been identified as having rotated clockwise at a relatively fast rate in the last 4 to $5 \mathrm{Ma}$ [Lamb, 1988; 2011; Rowan et al., 2005; Walcott, 1993].

cGPS sites MAKO and GISB are located on or very close to the boundary between the Wairoa and Raukumara domains, according to Fig. 1A in Rowan et al. [2005] and Fig. 5 a in Lamb [1988]. So-called "anomalous localities" in this immediate area on the coast east of Gisborne [Lamb, 2011] have given rise, from paleomagnetic data, to high estimates of rotation ( 8 to $14 \mathrm{deg} / \mathrm{Ma}$ ) in relatively recent times (late Miocene $\sim 8 \mathrm{Ma}$ ), but because of difficulties in geologically and tectonically interpreting these values, such results have tended to be contentious. Lamb [2011] suggested that:

"Some of the anomalous rotations could record local large vertical axis rotation of small blocks, especially in the region east of Gisborne, which is located where there is dextral shear and at the southern edge of a region of pervasive extension." (p.146)

However tempting it may be to draw parallels, we must be clear that apparent rotational components of average strain fields determined by geodesy or GPS measurements, over a decade or two, should not be confused with physical block rotations evidenced by geological or paleomagnetic evidence spanning more than a million years. As has been discussed, there is evidence to suggest that in the Raukumara region significant changes in strain fields take place on the scale of decades or less, which may reflect changes in locking as subduction evolves. In consequence, we should regard the information presented in Figs. 3.3d and 3.3e as a snapshot at a point in time, and, by analogy, any apparent differences in rotation rates shown should only be taken as indications of differing levels of strain field asymmetry over the period for which we have data. Over geological timescales, observed strains include the effects of SSEs. 


\subsubsection{Displacements due to SSEs or other discrete events}

Throughout this work, all offsets in de-trended cGPS displacement-time plots have been treated as potential SSEs. However, there are situations where this is unlikely to be the case - for example with respect to the immediate displacements and afterslip from the Cook Strait and Lake Grasmere events. Less clear is how the events around Mt. Ruapehu should be treated - whether as being volcanic in origin or as small-scale SSEs.

Whilst the main differences observed between surface manifestations of SSEs in the northeast and southwest relate to duration and periodicity, the other noticeable differences are in displacement amplitude (which may simply reflect the depth effect), and also, for displacements in the E direction of more than $4 \mathrm{~mm}$, the event speed - the events in the northeast appear to evolve approximately twice as fast (in terms of $\mathrm{mm}$ /day) than those in other parts of the margin (Fig. 3.2.3b). However, this could also be simply a depth-related effect - shallower events will give rise to larger surface displacements and will therefore appear to be progressing at higher speed. This is discussed further in 5.4.2.5.

\subsection{3 'Negative' short-term displacements}

Significant numbers (29\%) of E-component surface deformation events were detected that apparently moved in the opposite direction to that expected (e.g. westwards, rather than eastwards), and whilst the maximum amplitudes of these 'events' were generally smaller, they nevertheless in some cases had similar temporal evolution characteristics to SSEs. Five possible explanations present themselves:

1. 'Negative' displacements could, in some cases, represent a form of noise that has not been removed by the processing. For some of the very small 'events' seen at single sites, this could well be the case. It is also possible that, for example, in the volcanic region, there might be a contribution from sub-regional noise that has not been removed by processing.

2. 'Negative' displacements could arise from movement on faults dipping in the reverse direction to the subduction thrust and not necessarily directly associated with the subduction interface. The recently published model of active faulting in New Zealand [Litchfield et al., 2014] does show some candidate faults, although this catalogue largely comprises faults that can be found at or near the surface and 
which can be modelled in a regional scale. However, detailed interpreted seismic sections also show the likely presence of imbricate faults and backthrusts in the accretionary wedge [Barnes et al., 2010; Bell et al., 2014; Henrys et al., 2013; Mountjoy and Barnes, 2011] - see Fig. 5.2.3. Of course, there may also be buried faults that have not yet been discovered. In Appendix 14, modelling suggests that for imbricate faults with dip exceeding $60^{\circ}$, or for backthrusts with dip exceeding $40^{\circ}, \mathrm{E}$ component surface displacements measured to the west of the source would appear 'negative' (i.e. displacing westward). In Nankai, splay faults and imbricate faults within the accretionary wedge have also been suggested as sources of SSEs ${ }^{2}$ in addition to the décollement zone [E Davis et al., 2006; Ikari and Saffer, 2011]. This is therefore suggested as a possible explanation for several of the 'negative' events off the East coast, where inversions suggest source locations that are near the mapped locations of such fault systems. However, the moment magnitude of some of the negative events would imply significant slip on such relatively small faults as imbricate faults or backthrusts, and hence this explanation seems unlikely for the larger events.

3. Around Mount Ruapehu, surface displacements could be caused by slow movement on non-subduction faults, or some aspect of plutonic activity. Hydrothermal activity has been suggested to cause GPS 'events' at Taal volcano in the Philippines [Lowry et al., 2001a], but in our case, none of the GPS 'events' correspond with reported activity.

4. Thrust faulting with some component of strike-slip, and pure strike-slip faults could give rise to zones in which the surface displacement on the horizontal component would appear to be negative. This has been modelled and is discussed in Appendix 14. Whilst this mechanism should be resolvable in the inversions, in fact, inclusion of candidate 'negative' events in the few cases where this might be appropriate did not appear to significantly change residuals or the moment on temporally- or spatially-adjacent SSE inversions. These events have therefore been retained as separate entities.

5. It has also been suggested during the examination of this thesis [Lowry, 2015] that a likely possible explanation for negative events is that the megathrust experienced transient episodes of greater frictional coupling, and that such behaviour could be expected from rate-state dependent friction. This seems one of the more probable hypotheses.

\footnotetext{
$\stackrel{2}{2}$ The authors evidenced clay-rich zones at the fault locations from drill cores, and recovered gouge samples that behaved in laboratory experiments in a velocity-strengthening mode. They concurred with other authors' analyses suggesting that seismic slip is not expected to occur at shallow depths. They also note: "The velocity range over which we observe minimum values of $a b$ in all three fault zone settings $(1-3 \mathrm{~mm} / \mathrm{s}$, or $0.1-0.3 \mathrm{~m} / \mathrm{d})$ corresponds to estimated slip rates for slow slip events in both the Nankai area and on the San Andreas fault in California." (p12)
} 


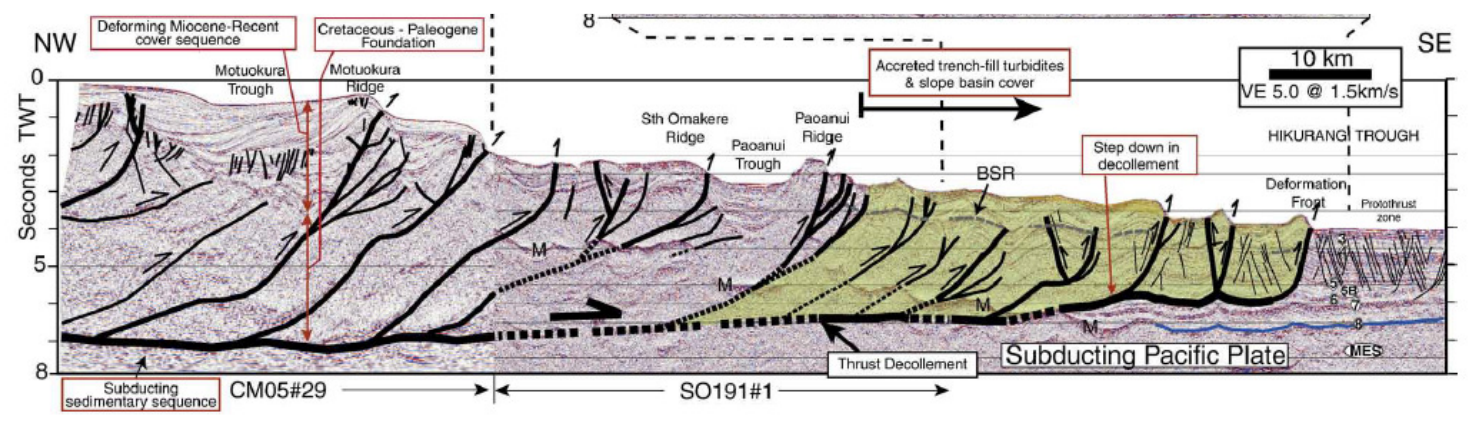

Figure 5.3.2 (reproduced from Barnes et al. [2010], Fig. 3): Interpreted multichannel seismic section across the central Hikurangi Margin (approx.: $-39.9^{\circ}, 177^{\circ}$ to $-40.6^{\circ}, 178.5^{\circ}$ ) illustrating imbricate faults and backthrusts. The seismic data are two spliced sections, including high-fold seismic data across the continental shelf (CM05\#29) and low-fold data across the slope and trough (SO191\#1). Note that structures illustrated are reproductions of interpretations originally made in detail on large sections with low $(2.5 \mathrm{x})$ vertical exaggeration. This transect is representative of the more classical accretionary structure and processes dominating the margin south of Rock Garden. M is the first seafloor multiple. The dotted lines in the lower centre of the low-fold profile are hypothetical extensions of the faults beneath the multiple.

In the few cases where 'negative' source slip values have been reported in the literature, these have been attributed to "uncertainties and errors associated with the inversion procedure" [Radiguet et al., 2011], or noise in the raw data [Bartlow et al., 2014]. We find that there are too many of these events, some of which are well-constrained, for these explanations to be considered satisfactory.

\subsection{Event displacement-time shapes (temporal evolution)}

There are two questions to be answered with respect to the analysis of 'event' displacement-time shapes:

1. Is there any theoretical or logical reason why SSE displacement-time curves should have a specific shape?

2. Could knowledge that displacement-time curves may have a distinctive shape assist in improving the detection of events? 


\subsubsection{Theoretical and observational background}

Friction models are now frequently invoked during discussion about the movement of faults [Scholz, 1998]. Despite the enormous differences in scale, laboratory experiments provide useful information. The time-dependent behavior of rock discontinuities, as determined in laboratory experiments, gave rise to the now-familiar rate and state equation, attributed to Dieterich [1978] and expressed in the following manner by Marone [1998] (p. 650):

$$
\mu=\mu_{0}+a \cdot \ln \left(\frac{V}{V_{0}}\right)+b \cdot \ln \left(\frac{V_{0} \theta}{D_{c}}\right)
$$

where:

\begin{tabular}{|c|c|}
\hline$\mu$ & $\begin{array}{l}\text { is the friction coefficient, or ratio of shear stress to effective } \\
\text { normal stress }\end{array}$ \\
\hline$\mu_{0}$ & is a constant appropriate for steady-state slip at velocity $V_{0}$ \\
\hline$V$ & is the frictional slip rate \\
\hline 9 & $\begin{array}{l}\text { is the state variable, sometimes interpreted as the characteristic } \\
\text { contact lifetime }\end{array}$ \\
\hline$D_{c}$ & $\begin{array}{l}\text { is the critical slip distance, regarded by Dieterich as the distance } \\
\text { required to renew surface contacts }\end{array}$ \\
\hline & al constants \\
\hline
\end{tabular}

The literature often refers to the difference between ' $a$ ' and ' $b$ ' as a material property that varies in the following manner: if $(a-b)$ is positive or zero, the material is said to be 'velocity-strengthening', and if negative, 'velocity-weakening'. The velocitydependence ('a' component) and aging (' $b$ ' component) of $\mu$ are said to be related behaviours that result from the creep of surface contact and a consequent increase in real contact area with time of contact.

Laboratory stick-slip measurements in suitably stiff servo-controlled equipment, on simulated faults, with varying degrees of roughness and gouge, produce displacementtime plots that demonstrate transitions from velocity weakening to velocity strengthening frictional behaviour, and the resulting plots appear similar to asymmetric sigmoids [Kaproth and Marone, 2013; Ohnaka, 2013]. Kaproth and Marone sheared layers of lizardite-rich serpentinite at constant normal stress of $1 \mathrm{MPa}$. Each experiment included more than 50 stick-slip events, with durations ranging up to $35 \mathrm{~s}$ (Fig 5.3.1a). Their experiments showed that the laboratory fault zones undergo a transition from 
velocity-weakening to velocity-strengthening friction behavior above slip rates of $\sim 10$ $\mu \mathrm{m} / \mathrm{s}$, and they suggested that SSEs could result from the rate-dependent frictional behaviour of the fault gouge itself.

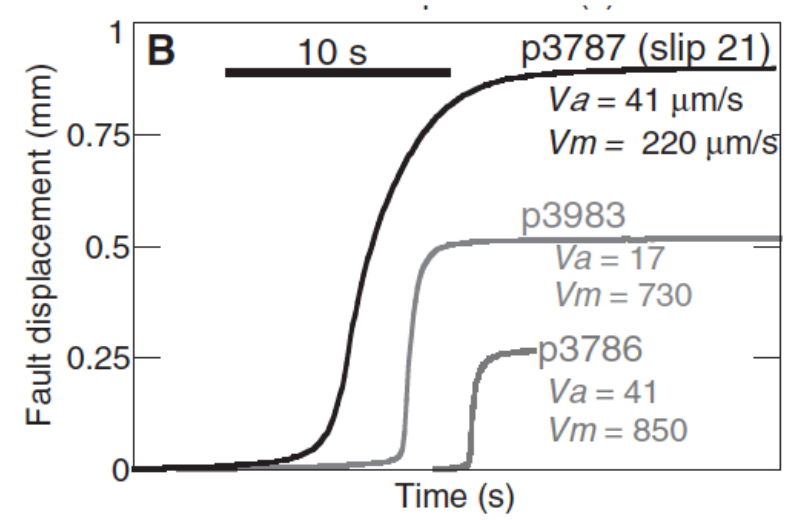

Figure 5.3.1a (Fig. 2B from Kaproth and Marone [2013]): Details of slip acceleration history for 3 representative stick-slip events with average slip velocities (Va) from 17 to $41 \mu \mathrm{m} / \mathrm{s}$. Maximum slip velocities $(\mathrm{Vm})$ for these samples ranged from 220 to $850 \mu \mathrm{m} / \mathrm{s}$, but the fastest velocities were only sustained for short periods $(<1 \mathrm{~s}-$ sufficient to mobilise velocity-strengthening). Acceleration and deceleration periods often lasted $10+\mathrm{s}$, resulting in low average velocities.

However, many authors draw attention to the fact that it is difficult to scale from the laboratory to a subduction interface, and point to the anomaly of laboratory-scale friction coefficients being up to 5 times the values needed to model the behaviour of subduction interfaces, e.g. Cattin et al. [1997].

Nevertheless, Yajing Liu, in her PhD thesis [Y Liu, 2007], whilst modeling the effects of small along-strike variations in constitutive rate and state friction parameters ' $a$ ' and 'a b' in a subduction zone model based on Cascadia, gave results that showed "spontaneous slip transients" (SSEs), in which the shape of the source displacement time curves [Y Liu and Rice, 2005] can be shown to fit an asymmetric sigmoid slip-time model. Whilst the time-scale of the transient shown in this 2005 paper is much longer than is normally observed, they subsequently noted that as the effective stress is reduced in the model (through pore pressure increases), the duration of the events was significantly reduced [Y Liu and Rice, 2007]. [Indirect evidence of high fluid contents or pressures in the interface zone of the northern section of the Hikurangi subduction have been presented by a number of authors [Bassett et al., 2014; Bell et al., 2010; Davey 
and Ristau, 2011; Eberhart-Phillips and Reyners, 2012; Fagereng and Ellis, 2009; Heise et al., 2012; Reyners and Eberhart-Phillips, 2009]].

$Y$ Liu and Rice [2007] suggested that using a rate and state model, the fault response can be characterized by a parameter $\mathrm{W} / \mathrm{h}^{*}$, where $\mathrm{W}$ represents the length up-dip of the unstable region over which elevated pore pressures exist, and $h^{*}$ represents the stable patch size for steady sliding, as illustrated in their simplified 2D model cross-section (Fig. 5.3.1b).

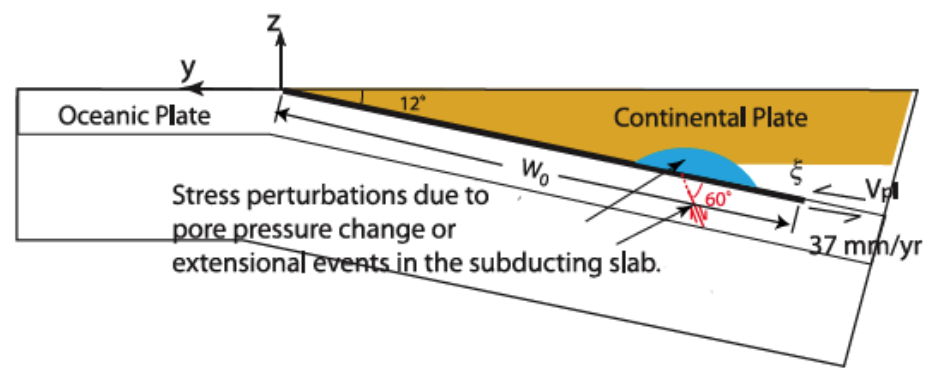

Figure 5.3.1b (Fig. 1 from $Y$ Liu and Rice [2007]): Two-dimensional subduction model. The parameter $\mathrm{y}$ is the horizontal direction opposite to the plate convergence, $\mathrm{z}$ is the upwards direction and $\mathrm{x}$ is in the along-strike direction, perpendicular to the y-z plane. A rate- and state-dependent friction law is applied to the fault $0<\xi<\mathrm{W}_{0}$. A uniform motion at plate convergence rate $\mathrm{V}_{\mathrm{pl}}$ is imposed at $\mathrm{W}_{0}<\xi<+\infty$. Stresses resulting on the thrust fault due to an external event in the subducting slab, or pore pressure changes from dehydration fluid release are considered possible sources of perturbation, as discussed in section 4 of their paper.

Their model was subsequently improved with the addition of fault gouge dilatancy strengthening, such that the maximum velocity during SSEs has been brought much more in line with observation [Y Liu and Rubin, 2010].

Similar modelling work has been carried out by Matsuzawa et al. [2010] showing that both short- and long-term episodic slow slip events can arise, using rate and state frictional models, although in this case the shape of the events cannot be discerned in the published paper.

Later work, using similar modeling philosophy, suggests that geological inhomogeneity along the boundary could be the source of frictional inhomogeneity, which, it has been suggested, could diminish the need for postulated changes in rate and state parameters [Skarbek et al., 2012]. An inhomogeneity mechanism has been suggested to explain, 
and used to model, periodic creep transients, similar to SSEs, on Californian strike-slip faults subsequent to earthquakes [Wei et al., 2013].

Y Liu and Rice [2009] also demonstrated that using a set of rate and state frictional parameters appropriate to the geology is important for effective modeling - they compared modeled behaviour against GPS measurements and concluded that for Cascadia, data based on gabbro (more similar to basaltic oceanic crust) gouge gave lower residuals than the originally-used laboratory data based on granite gouge (for lack of any better data at the time).

Whilst not directly related to the Hikurangi, but further south along the plate boundary and still with respect to gouge and rate and state friction models, recent work has shown the ability of cataclastic Alpine Fault materials to form gouges with both velocityweakening and velocity-strengthening behaviour [Boulton et al., 2012]. Velocitydependence of frictional behaviour of fault material has been the subject of intensive laboratory work in recent years [Campione and Capitani, 2013; den Hartog and Spiers, 2014; Kaproth and Marone, 2013; Katayama et al., 2013; Tsutsumi et al., 2011; Ujiie et al., 2013]. Rubin [2011] summarises the main features of the principal conceptual models, and episodic SSE slip propagation observations in Cascadia and Japan, and attempts to formulate a constitutive friction law that would allow different propagation rates along strike and up-dip.

Clearly, rate and state models, by their innately transformative formulation, lend themselves to the idea that displacement-time shapes could take on asymmetric sigmoidal form. In observational terms, data reported for the 2006 Guerrero SSE suggests sigmoidal forms in the de-trended GPS daily solutions, and the source-time function adopted by the authors has a clear asymmetric sigmoid form using 4 parameters (amplitude, initiation time $\mathrm{t}_{0}$, and accelerating and decelerating rise times) [Radiguet et al., 2011]. The authors suggest that:

"Our results show that the slip evolution during the SSE can be described with a rather simple smooth ramp. The rise-time of this slip is large with respect to the total duration of the rupture process, which means that there is an interaction between large parts of the fault during the dynamic process." (p.827) 
The "simple smooth ramp" model of Radiguet et al. [2011] is attributed to P Liu [2006], in which is detailed a special case of the more general functional equation used for the time-evolution of slip on a fault in an earthquake [P Liu and Archuleta, 2004]:

$$
\frac{\partial S(t, r, p)}{\partial t}=C\left(\frac{t}{r}\right)^{p}\left(1-\frac{t}{r}\right)^{5-p}, \quad 0<t<r,
$$

where $r$ is rise time or duration of slip; $C$ is a normalizing constant such that $S(t)=1$ when $t \geq r$; and the exponent $p$ controls the shape of slip rate function. The exponent $p$ can vary from 1 to 4 . In the particular case $p=1$ this slip rate function has an amplitude spectrum that is practically identical to the Aki-Brune $\omega$-squared model [Aki, 1967;

When numerically integrated (by iteration), it can be shown that this equation generates asymmetric sigmoidal forms in the displacement-time domain. The equation has the benefit of a limited number of parameters $-\boldsymbol{r}$ is simply the duration of the event, $\boldsymbol{C}$ is the inverse of the maximum of the integrand $S$, and $\boldsymbol{p}$ governs the shape of the curve.

Earlier authors perhaps did not consider asymmetric sigmoid source models simply because of the paucity of either high-quality near-field seismic data, or adequate GPS data, that would allow discrimination between symmetric and asymmetric functions. Two examples of where symmetric sigmoids (generated with modified cosine functions) were used as slip source in earlier papers, referred to by $P$ Liu and Archuleta [2004], concern the 1992 Landers strike slip event [Cotton and Campillo, 1995], and the complex 1994 Northridge and 1999 Hector Mine events [Ji et al., 2002]. More recently, Fukuda et al. [2014] analysed the temporal evolution of the 2013-14 Boso SSE, and their data, obtained using a modified network inversion filter, show the asymmetric sigmoidal shape. They divide the evolution into 2 phases, noting a lack of swarm activity in the early part of the curve where propagation speeds were low, suggesting stress loading, followed by accelerating slip associated with increased seismicity, which then decelerated rapidly. They question whether the early part of the evolution had been undetected in earlier work. They also draw the parallel with the outcome of rate and state modeling.

It should be noted that even though the above discussion points in the direction of a rate and state friction model (which is in itself an empirical construct) possibly providing a causal basis for the shape of some displacement-time curves, this should perhaps only apply in the case where the interface can be approximated by a planar sliding surface. 
In practice, seismic evidence points to some parts of the northern section of the Hikurangi subduction interface including major topographic anomalies such as seamounts [Barnes et al., 2010; Bell et al., 2010], for which $K$ Wang and Bilek [2014] argue that such planar frictional modeling is inappropriate. Indeed, they indicate that topographically rugged surfaces are more closely associated with subduction creep and SSEs, rather than smoother surfaces, which appear to be associated with megathrust earthquakes. Pacheco et al. [1993], after studying the seismic coupling coefficient for 19 plate interface zones over a 90 year period, suggested in a similar vein that subducting bathymetric anomalies could, along with pore fluid pressure and frictional heating, be contributory factors to some zones exhibiting very low coupling and lack of very large earthquakes. However, subsequently McCaffrey [1997] argued that the uncertainties associated with seismically-determined coupling coefficients are generally large, and thus, except for the very low values, most can be considered statistically equivalent to a mean value of 0.3 .

Scholz $[2002]^{\underline{3}}$ suggested that subducting seamounts could also result in localized high coupling through increased normal stress, citing the example of the 1994 Java earthquake, which was attributed by Abercrombie et al. [2001] to slip on part of a subducted seamount. The patch was assessed as having been locked from the seismic data, but otherwise surrounded by an uncoupled aseismic interface - which brings us back to the penultimate paragraph in section 1.6: "spatial frictional heterogeneity may play a large part in explaining variation in seismic and aseismic behaviour on large faults".

${ }^{3}$ It is worth noting that there is often confusion about the coupling coefficient. Scholz warns that a locked patch has a coupling coefficient of 1 and a sliding patch has a coupling coefficient of 0 . "If some region such as a subduction zone has a seismic coupling coefficient of 0.5 , this does not mean that for the entire region half its slip is seismic and the other half aseismic.... An area of the conditionally unstable regime can have a coupling coefficient between 0 and 1 , so the correct interpretation is that something less than half the sliding area in the entire region is in the unstable regime." ( $\mathrm{p}$ 341) It can also be more widely argued that any value of coupling coefficient between 0 and 1 should be regarded as an "apparent coupling coefficient" [Hyndman, 2007] since it must be the result of a determination in which the sample is an average, or is biased (e.g. by an incomplete catalogue) in either time, or space, or both. 
With respect to earthquake dynamics, Udias et al. [2014] point out that:

"The main problem with classical rate and state friction in earthquake dynamics is that the experimental values of the parameters ' $a$ ', ' $b$ ' are typically of the order of 0.01 to 0.02 and $L$ is of the order of a few microns." (p242)

They then argue that such values do not correspond with estimates from data for earthquakes of magnitude $\mathrm{M}_{\mathrm{w}} \sim 7$. However, since the event timescales (and hence slip velocities) are quite different, earthquakes and SSEs may be governed by different frictional mechanisms.

Conversely, however, Kaneko et al. [2010], from their modeling of a "typical" subduction zone, argued that rate and state friction models, taken together with spatial frictional heterogeneity, are capable of predicting the mix of earthquakes, spatially variable interseismic coupling, creep and SSEs commonly observed in subduction zones.

In summary, there is evidence that along the northern, central and southern parts of the Hikurangi margin, interface macro-texture ranges from rugged to smooth, with significant variation in apparent coupling coefficients, yet we have observed displacement-time shapes that have a degree of commonality along the margin. Rate and state friction models may provide a basis for modeling SSEs, but other possibilities exist.

\subsubsection{Improving detection}

If it is accepted that the form of surface displacement-time plots arising from SSEs could be modeled by asymmetric sigmoids, how can this help in the detection process? Eq. 3.2.5 shows that this form requires 4 parameters, which relate to amplitude, duration, and degree of asymmetry. Whilst it was indicated in 3.2.5 that there was some correlation between the ' $b$ ' and 'c' parameters, and ' $a$ ' is simply a scaling factor that represents the total amplitude, it is the $4^{\text {th }}$ parameter that is of interest. Parameter ' $\mathrm{d}$ ' is non-linear in its effect: values between 0 and 1 indicate curves that have a short start and a longer finish, whereas values above 1 (up to $10^{18}$ in our case) represent curves that have a long start and a shorter finish. Reference to table 3.2.5 shows inconsistency, in 
that even for 'events' for which the residual errors of fit are small $(\sim 5 \%)$, the values of ' $d$ ' for stacked and individual results can be quite different - for example, event 41, where ' $\mathrm{d}$ ' is $\sim 0.1$ on the stacked data, but $\sim 10^{15}$ on the individual displacement results from the site with the largest amplitude. These ' $d$ ' values indicate two quite different curve shapes for the stacked and largest individual site result, even though both are of the AsymSigR form. This suggests that individual site records for an event could have a variety of shapes, and despite the preponderance of the generic AsymSigR form, this indeed proves to be the case (Fig. 5.3).

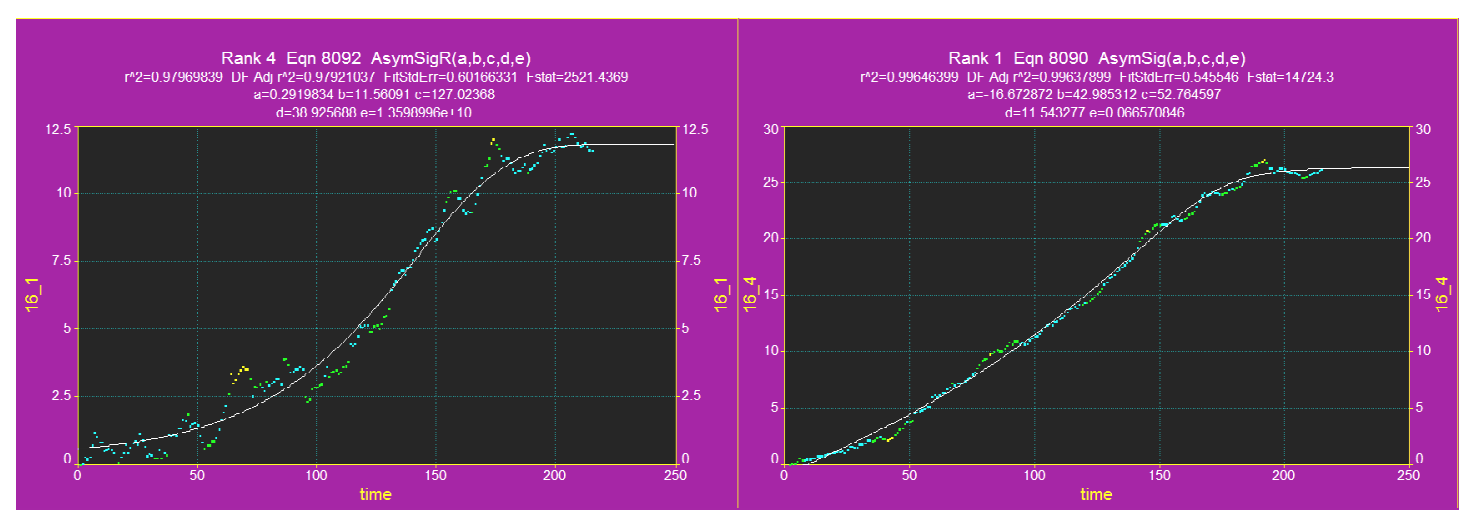

Figure 5.3: Examples of 2 individual displacement-time site records for 'event' 16, illustrating that whilst in both cases there is a very good fit to the asymmetric sigmoid form, the shapes are quite different, with 'd' coefficient values of $1.4 \times 10^{10}$ and 0.07 respectively.

This therefore suggests that even if the shape of each curve contains some valuable sitespecific information (such as spatiotemporal evolution of the slip patch), which has yet to be determined, it is not of any value in designing a universal template for event detection. It could, however, be used in a network sense for repeated events [Foster et al., 2013]

One potential simplification would be to remove the asymmetry. Automated search using sliding windows with hyperbolic tangent fitting has been utilized by some workers [Brudzinski and Allen, 2007]. The technique is described by Larson et al. [2004], and effectively reduces the number of parameters from 4 to 3, somewhat simplifying the search algorithm. 
It must be concluded, therefore, that any attempts at automated event detection will probably need to be based on simpler techniques, such as those described in the last paragraph, or criteria based on changes in standardized moving average gradients, or methods such as those commonly used in quality control: the Shewhart or more sensitive CuSum methods, or a combined method [Abujiya et al., 2013] may also be appropriate. Further work is required on this issue.

\subsection{SSE catalogue}

\subsubsection{Comparison with published results}

Appendix 15 tabulates comparisons between inversion results from this project and published results in Table 2 of Wallace and Beavan [2010]. As indicated earlier, the catalogue is regarded as preliminary, since additional work is ongoing to improve the slip area delineation. Nevertheless, and bearing in mind that Wallace and Beavan split the Manawatu 2004 and Kapiti 2008 events into stages that do not exactly correspond with the way in which we have identified discrete events, the level of agreement is considered good for the 16 events that could be directly compared:

- On average, there is no statistically significant difference in location centres. The standard deviations of the paired differences for latitude and longitude are $0.21^{\circ}$ and $0.15^{\circ}$ respectively, approximately corresponding to \pm 30 to $40 \mathrm{~km}$ at the $95 \%$ confidence level. Since we have no information concerning the uncertainty of the published results, we cannot partition the variance. Where 95\% confidence levels have been calculated for our locations, these typically average $\pm 28 \mathrm{~km}$.

- On average, there is no statistically significant difference in equivalent moment, when compared with either the 'disloc99' or 'DEFNODE' values, although overall, the closest agreement was with the 'DEFNODE' values. However, some of the individual comparisons show significant differences, with event 44 showing the highest percentage difference between our results and the 'DEFNODE' value. On average, compared to the 'DEFNODE' values, our results were $14 \%$ lower, but with a standard deviation of $50 \%$, this value is not significantly different to zero at the 0.05 level. Many of the differences significantly exceeded the $95 \%$ confidence limits on our moment estimates, but since there were no corresponding uncertainties associated with the published estimates, it is not possible to establish whether the individual differences are statistically significant. 
- In comparing the moment magnitudes, $\mathrm{M}_{\mathrm{W}}$, the overall average difference of 0.1 units (with an s.d. of 0.17 units) is not significantly different to zero at the 0.05 level. The maximum difference was 0.3 units. See Fig. 5.4.1.

- Given the limitations of the preliminary modelling used on this project, it is not surprising to find that our results for slip and area are on average 30\% larger and $30 \%$ smaller respectively than the values tabulated by Wallace \& Beavan. This is probably partly due to the limitations imposed by using only square slip areas, and partly due to the trade-off between slip and slip area, since modelled slips are relatively insensitive to changes in area.

- For the northern events, which are of relatively short duration, most of our durations are longer than those tabulated by Wallace \& Beavan. This is almost certainly a reflection of different methodologies used to define the beginning and end of the events. In this project, the earliest start time and the latest finish time over all sites, at which the event was observed, have been used to define the event duration. Usually the finish time is relatively poorly defined in the individual site cGPS data.
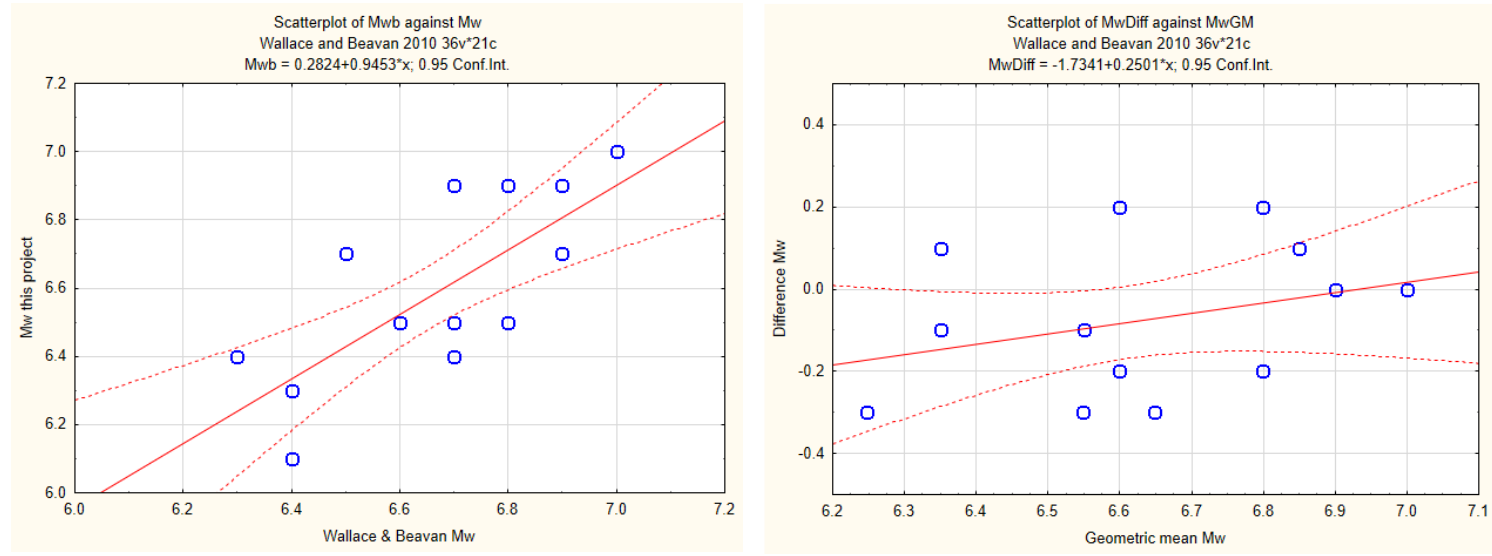

Figure 5.4.1: Comparison of Magnitude moments (Mw) from this project and from the 16 events that are directly comparable in Wallace and Beavan [2010]. Note that because of rounding, 3 comparisons plot coincidently. The left plot shows a conventional analysis. However, because both variates have significant uncertainty, ordinary least squares regression gives a biased result. The plot on the right shows the differences plotted against the geometric means of the two values, which is an appropriate plot for this type of comparison (since $\mathrm{Mw}$ is a logarithmic transformation) [Bland and Altman, 1999]. In the conventional plot, the 1:1 relationship would fit within the (biased) $95 \%$ confidence limits of the regression. In the Difference versus Average plot, a mean difference of 0.0 would just fit within the (unbiased) $95 \%$ confidence limits of the regression. Within the limits of this small data set, there is therefore no evidence to suggest that the two sets of results are significantly different at the 0.05 level.

\subsubsection{Statistical analyses}

It should be noted in all these analyses that the cGPS network was in the process of development for much of the time period covered by this project, and therefore coverage is biased with respect to the time history. 


\subsubsection{Slip patch distribution}

Fig. 3.4 shows the geographic distribution of the 139 estimated non-volcanic and nonexcluded sources from the inversion. The amount of information in the plot is potentially confusing, but it can be seen that the model shows events located from offshore of the East Cape and decreasing in number through to the top of the South Island. However, there appears to be a gap, or at least a zone of very diminished activity, along the east coast in the region offshore of central to south Hawkes Bay, and there are also slightly fewer events in the Manawatu region. A histogram of location latitudes (Fig. 5.4.2.1a) lends some credibility to this observation, but a more thorough statistical analysis is required to elaborate further (e.g. Mousavian and Hossainali [2014]). Segmentation and gaps between segments of SSE activity have been noted in SW Japan [Obara, 2010], and Cascadia [Brudzinski and Allen, 2007], and the latter authors suggest that the properties of the overlying plate may play a part in this. Audet and Bürgmann [2014] also suggested that recurrence intervals of SSEs increase linearly with the average ratio of $v_{p}$ to $v_{s}$ of the overlying forearc crust. Further work is required to study these aspects and any relationship to subducted geometry.

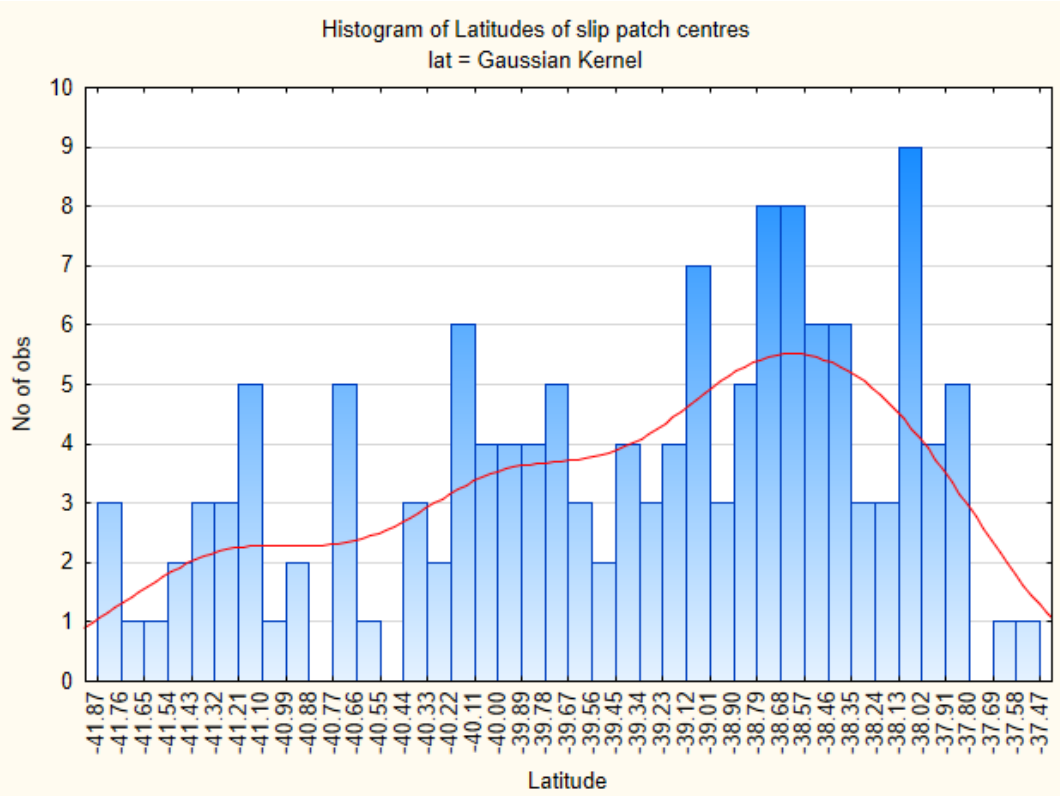

Figure 5.4.2.1a: Distribution of slip patch centres with respect to Latitude, with a superimposed Gaussian Kernel fit, showing greater numbers of events in the north, diminishing towards the south. The Gaussian Kernel fit shows a smoothed "average", but the raw data in the histogram suggests lower numbers to the south of Hawkes Bay ( -39.3 to -39.6) and in the southern Manawatu ( -40.4 to -40.8). 
Whilst the depths of slip patches are constrained by the geometry of the subduction interface, it is constructive to review the range of depths from the inversions, if only to compare with the summary of existing information referred to in section 1.6 (where it is noted that there has been a gap in the depths between shallow and deep events). As shown in Fig. 5.4.2.1b, this does not seem to be the case.

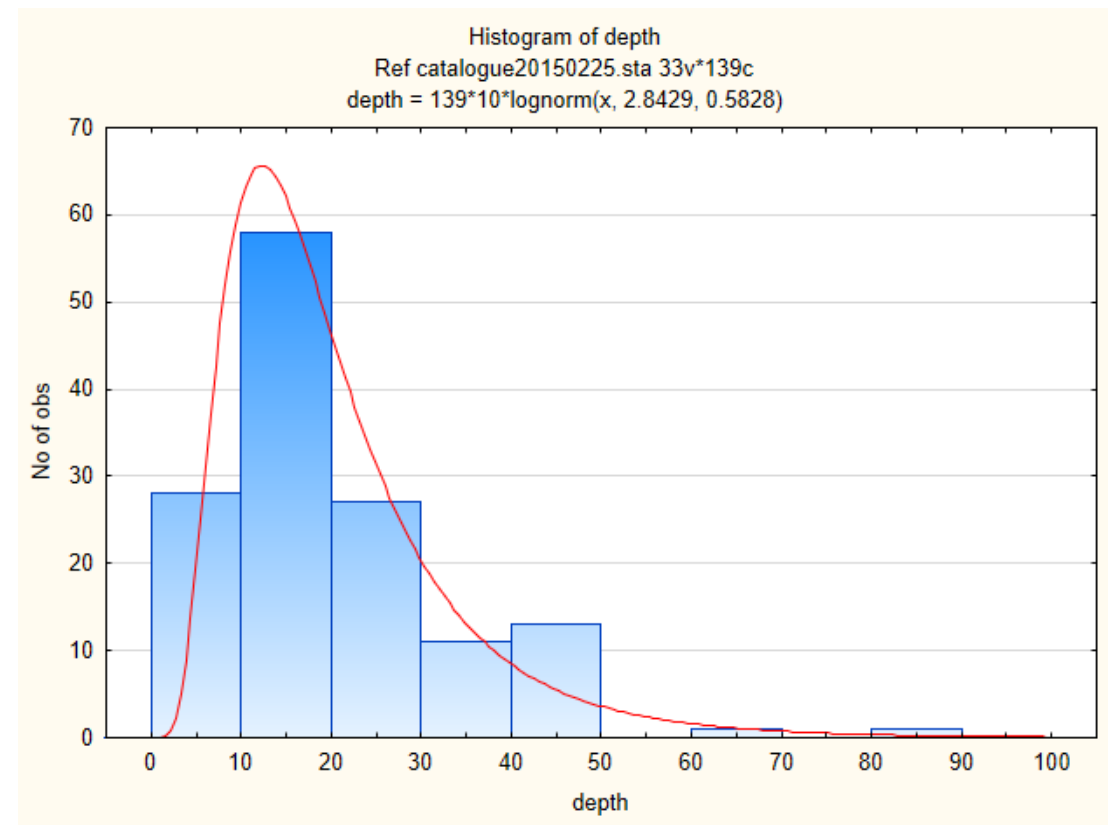

Figure 5.4.2.1b: Histogram of source slip depths $(\mathrm{km})$ arising from the inversions, fitted with a lognormal distribution.

\subsubsection{Slip orientations}

The inversion code does not make any assumptions or place any constraint on the orientation of the slip direction since dip-slip and strike-slip source slips are estimated independently (with the constraint that their combined squared residuals should be minimized). Slip orientations are plotted in Fig. 5.4.2.2a on the rotated NZTM coordinate system, and it can be observed that the majority of slips appear to be directed approximately perpendicular to the trench, and in some cases parallel to the trench, but with less in the intermediate angles. Figs. 5.4.2.2b and c summarise this information in two different ways. 


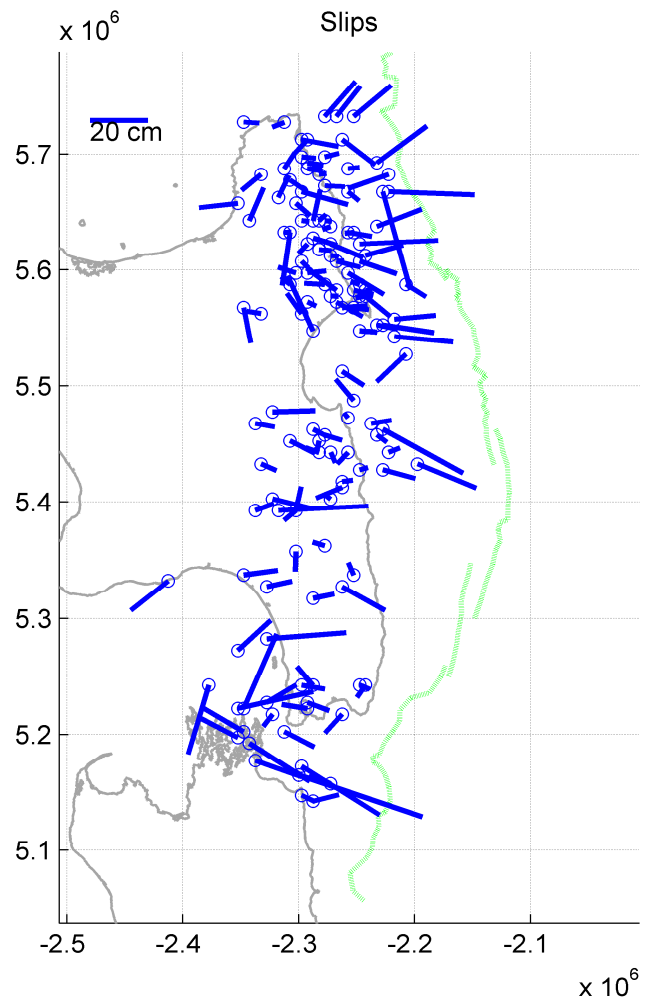

Figure 5.4.2.2a: Slip centres and orientations plotted on rotated NZTM coordinates. Compare Fig. 3.4.

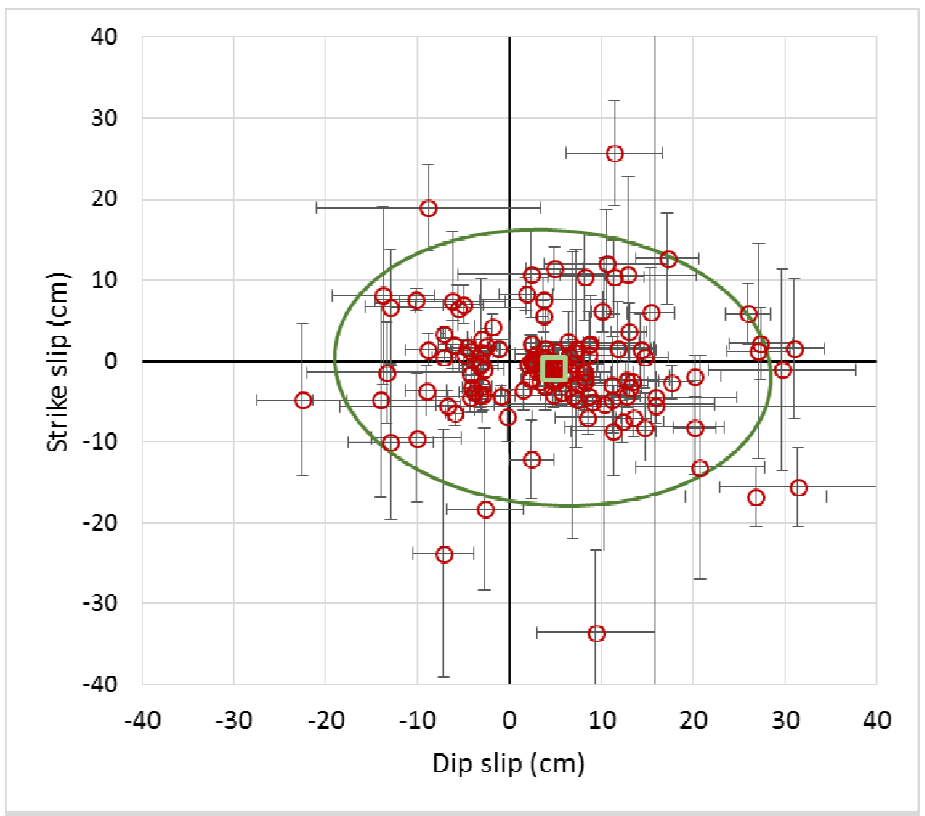

Figure 5.4.2.2b: Slip strike and dip components plotted to show the relative amplitudes and uncertainties in each component as $95 \%$ confidence limits. The superimposed ellipse indicates the $95 \%$ boundary for the orientations, with the major axis lying approximately along dip, and the minor axis along strike. Note that strike and dip are defined with respect to the mean strike of the margin (042 with respect to grid North) 


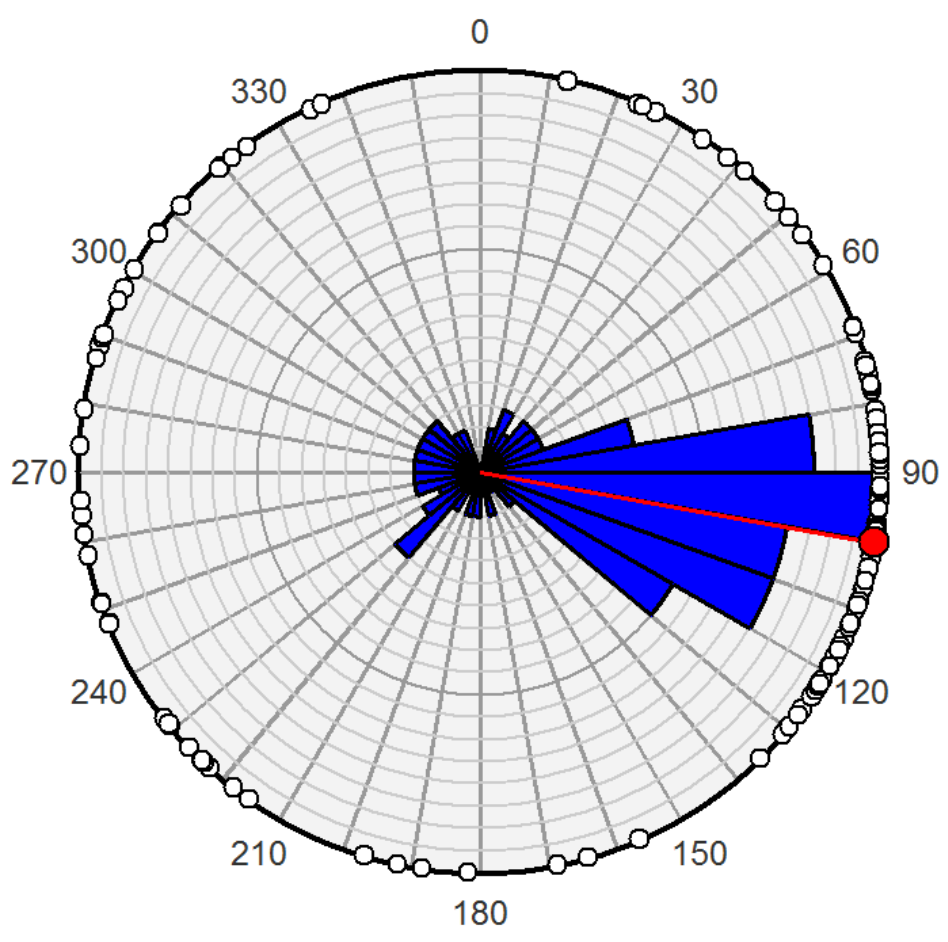

Figure 5.4.2.2c: Histogram of slip azimuths plotted with respect to the mean strike of the margin, using Orient software [Vollmer, 2012]. This illustrates the predominance of slip orientations within -10 to $+30^{\circ}$ of the subduction interface dip.

The results show that the majority of the modeled SSE slips are oriented within -10 to $+30^{\circ}$ of the subduction interface dip. The 'negative' events (i.e. hanging wall slipping downdip) cover a wider distribution of azimuths.

These results may be compared with Fig. 7 in Wallace and Beavan [2010]. Most of their slip vectors, derived using 'disloc99', also point in the direction of the trench, except in the Manawatu and Kapiti areas, where their vectors are aligned in a more ENE direction. Reference to Fig. 5.4.2.2a shows that this is not greatly at variance with some of the vectors for the larger events that we have determined in these areas (bearing in mind that our figure is rotated $42^{\circ}$ anticlockwise to theirs).

\subsubsection{Slip areas}

The seismic moments of the events from the model scale with slip area (see Fig. 5.4.2.2a). However, moments arising from the inversions were generally insensitive to large changes in area because of compensating changes in model slip, and in 
consequence operator intervention was arbitrarily used to select patch sizes so as to maintain slip components below approximately $30 \mathrm{~cm}$.

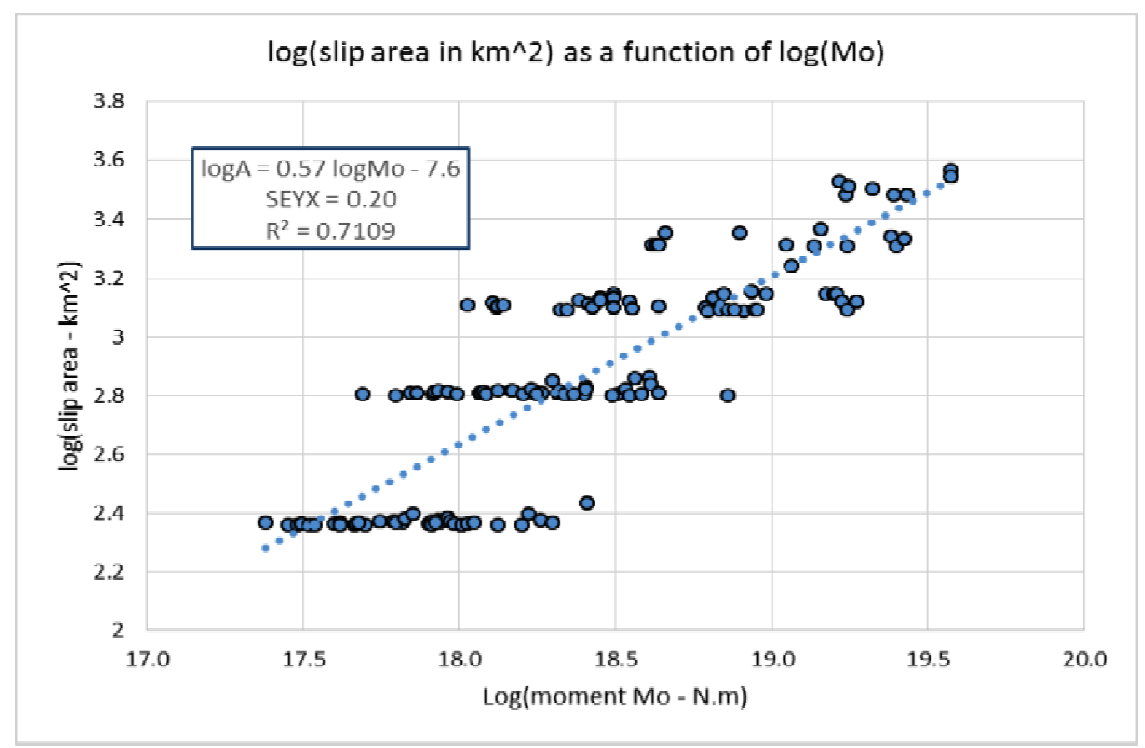

Figure 5.4.2.2: $\log \left(\right.$ slip area in $\mathrm{km}^{2}$ ) plotted against Log (moment). The discrete nature of the slip areas is an artifact of the selection process, in which the model was limited to square slip patches (on the surface projection). Whilst an ordinary least squares regression equation is shown, this is for indication only since it is biased because the two variates are not independent.

Despite the limitations noted, in Fig. 5.4.2.2a it will be observed that the gradient of the regression is indicated to be $0.57+/-0.2$, and whilst this may be biased, it is not significantly different from the value of 0.65 for SSEs indicated by $H$ Gao et al. [2012]; $Y$ Liu [2014], or 2/3, which is the scaling for earthquakes.

\subsubsection{SSE durations}

A number of authors have suggested that the relationship between the duration of SSEs and the seismic moment is different to that for 'regular' earthquakes [H Gao et al., 2012; Ide et al., 2007; Y Liu, 2014; Peng and Gomberg, 2010], and their data suggest an overall gradient of approximately unity between $\log ($ duration) and $\log (\mathrm{Mo})$. Fig. 5.4.2.3a shows the data obtained in this project, and whilst the gradient of the ordinary least squares regression is significantly biased due to large variance in both variates, it seems unlikely that it is close to unity. An alternative geometric mean (functional) 
regression is also shown, and whilst this could also be biased, it is likely to be less so [Brace, 1977; Clarke and Van Gorder, 2013; Xu, 2014].

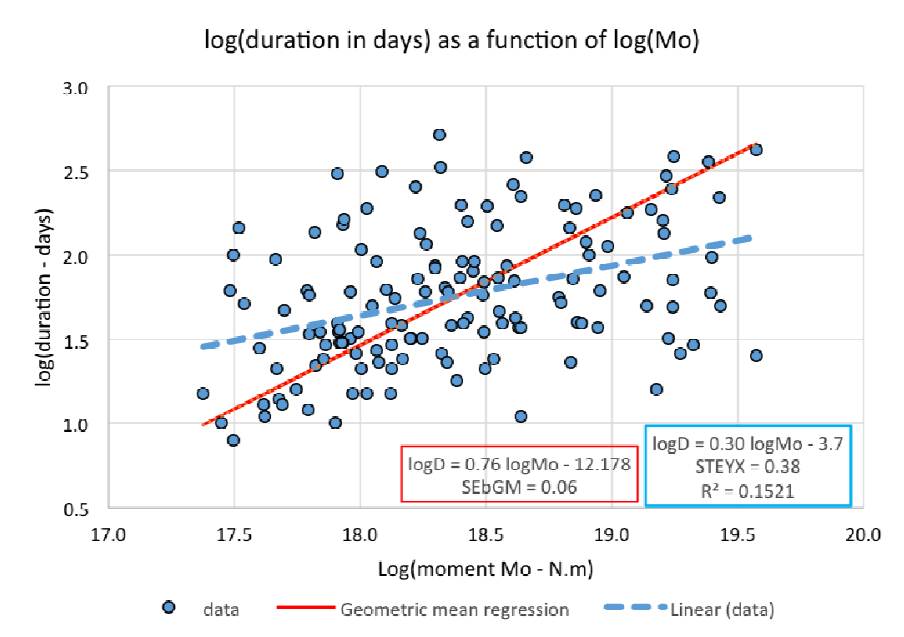

Figure 5.4.2.3a: Log-log plot of event duration against moment (Mo). The ordinary least squares regression is shown for information (dashed blue line with statistics in blue-lined box), but it should be noted that this gradient is biased because there is significant variance in both variates. An alternative, less biased, geometric mean (functional) regression is shown by the red line (statistics in red-lined box).

However, if our data is compared with that summarised by the authors noted above, and in particular Fig. 3 of H Gao et al. [2012], it can be observed that it is consistent with the generalized relationship expected for SSEs (Fig. 5.4.2.3b).

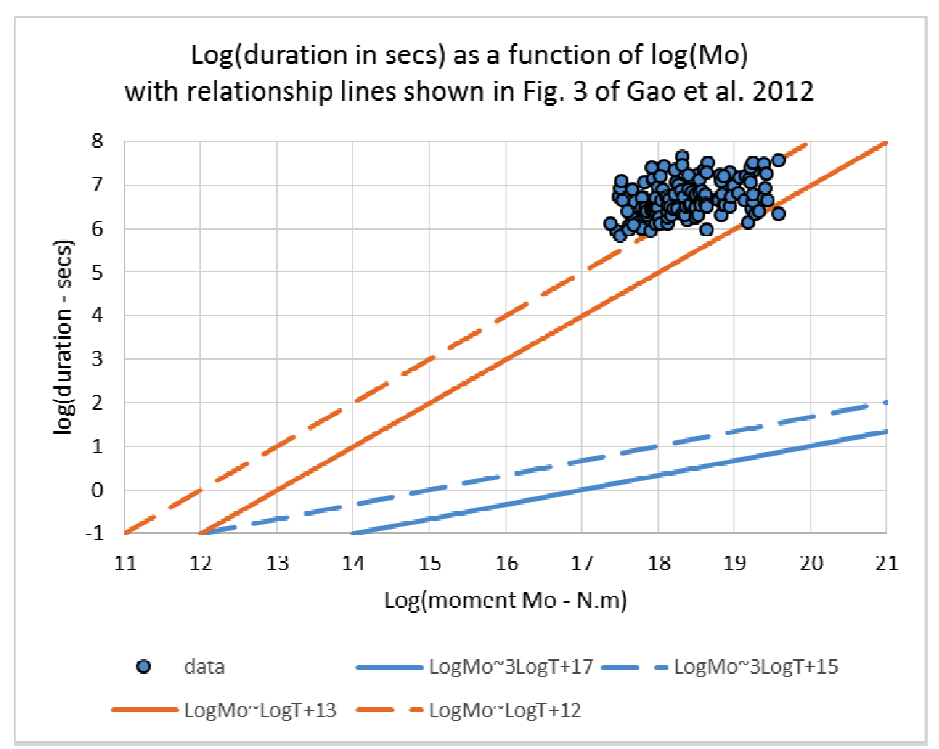

Figure 5.4.2.3b: Comparison of event durations and moments in the same format as Fig. 3 of $\mathrm{H}$ Gao et al. [2012]. The two lower (blue) lines indicate approximate boundaries of the relationship expected for conventional earthquakes, whilst the two upper (orange) lines indicate two similar indicative trend lines suggested for SSEs by authors referenced in the text. 
Furthermore, figure 3 of $H$ Gao et al. [2012] shows a group of slow earthquake data from Cascadia, which, whilst fitting overall within the indicative trend boundaries of $\log ($ duration $) \sim \log (\mathrm{Mo})$ has an apparently similar gradient within the group to that shown in Fig. 5.4.2.3a - however, they suggest that "the lower slope characteristics of data from a particular study or geographic region may represent an artifact, potentially reflecting the saturation of a fault parameter from limited observations or the methodology."

Peng and Gomberg [2010] indicated that with a wider variety of data, the separation between the scalings of Mo $\sim \mathrm{T}^{3}$ (a gradient of 0.3 ) and Mo $\sim \mathrm{T}$ (a gradient of 1 ) may become more of a continuum rather than 2 distinct sets of scaling laws. They suggest that: "Two distributions clearly exist in the data, but we suggest that they are observational, comprising either geodetic or seismic measurements. These are separated by a gap in duration of more than two orders of magnitude. This gap may be attributed partly to instrumental limitations."

However, Y Liu [2014] suggests that the situation is more complex but amenable to modeling, noting that "scaling between event duration and equivalent moment is diagnostic of the underlying physics of the rupture process". Liu's modeling within the framework of rate-state friction indicates a gradient close to 0.5 for a variety of fault geometries with dips between 15 and $30^{\circ}$ and strike lengths between 480 and $1024 \mathrm{~km}$. This would be within the bounds of feasibility for the data shown in Fig. 5.4.2.3a.

\subsubsection{Moment distribution}

The size distribution of earthquakes is commonly portrayed using a log-log plot of cumulative number against seismic moment. The common expectation from the Gutenberg-Richter (G-R) relationship [Gutenberg and Richter, 1944] is that such a plot should show a linear relationship (exponential distribution) with a gradient close to -1 . The G-R relationship is often expressed as:

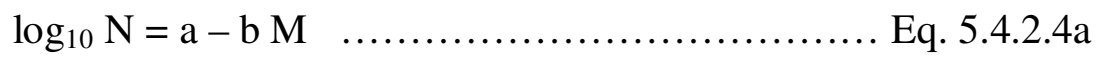

where: $\mathrm{N}$ is the number of earthquakes of greater than or equal to magnitude $\mathrm{M}$, and ' $\mathrm{a}$ ' and ' $b$ ' are constants. 
There are a number of well-known issues with this simple relationship: the finite amount of energy available to generate earthquakes means that there has to be some modification of the relationship at high magnitudes; and catalogue incompleteness at low magnitudes due to network detection sensitivity means that there will be some "roll-off" in the apparent linearity of the log-log relationship. There are a number of other significant potential systematic and statistical issues that could also bias the value of 'b' [Kagan, 1991]. Kagan proposed that in order to eliminate at least one source of uncertainty it is preferable to work with seismic moment rather than magnitude.

In subsequent work, Kagan [2002] examined the statistical aspects in more detail and proposed methods for determining the (lower) cutoff moment and the (upper) corner moment of the distribution, so that the $\beta$ value for the intervening magnitude range could be accurately determined. He tested a number of distributions and suggested the use of the Tapered G-R (Pareto) distribution (TGR), which in the probability density function format is expressed as:

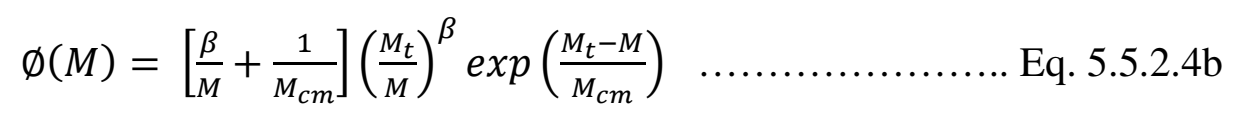

where: $\beta$ is the index parameter of the distribution $=0.667 \mathrm{~b}$

$\mathrm{M}$ is the scalar seismic moment

$\mathrm{M}_{\mathrm{cm}}$ is the corner moment (upper)

$\mathrm{M}_{\mathrm{t}}$ is the catalogue completeness threshold moment (lower)

His proposals for determining the corner moment (upper limit) by maximum likelihood estimation, together with a method for determining the rolloff in $\beta$ with changes in the cutoff moment, have been incorporated in Matlab code by Prof. E. Smith and utilized to estimate $\beta$ and ' $b$ ' values for our catalogue.

The principles of the process are illustrated in Figs. 5.4.2.4a-c for the 139 events that are non-volcanic and not excluded (see 3.4). 

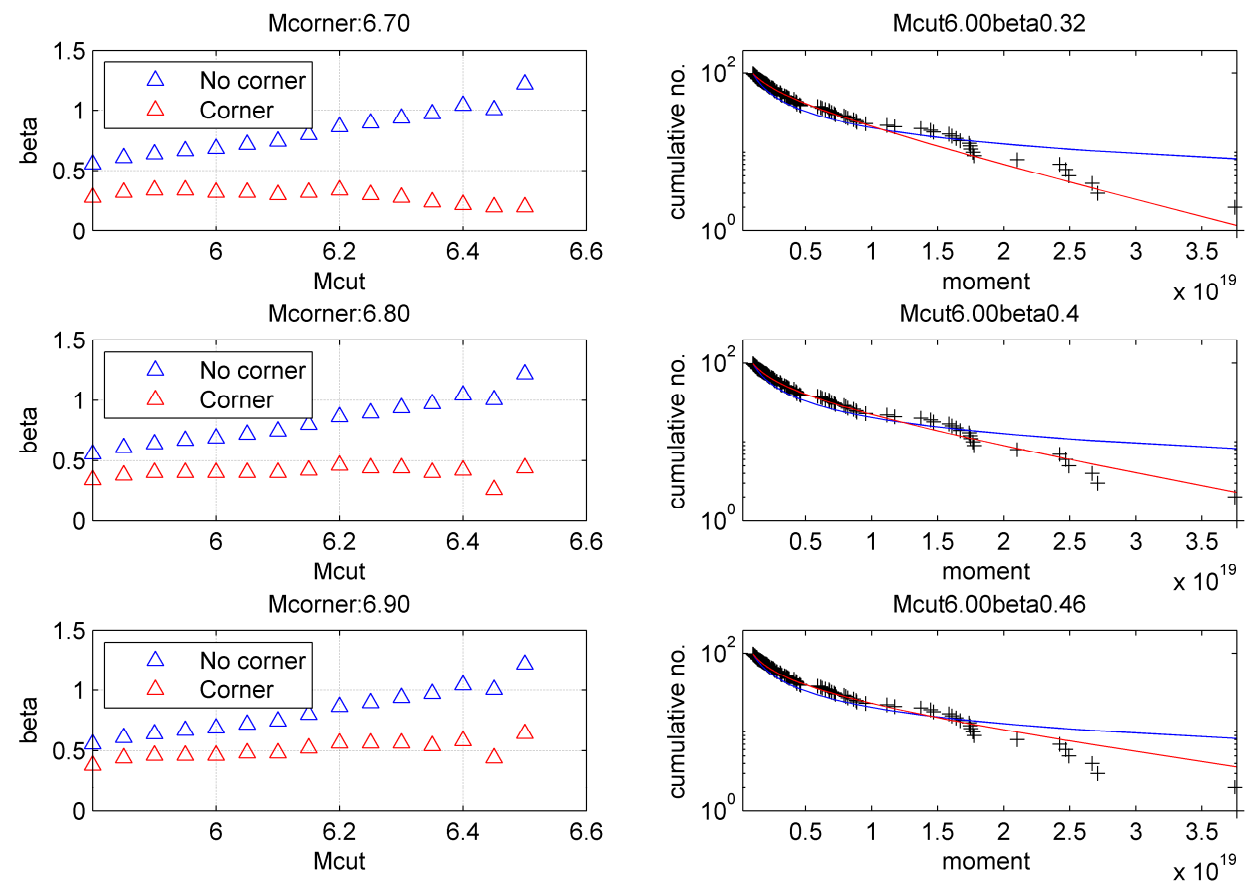

Figure 5.4.2.4a: Plots illustrating the effect of changing the equivalent corner moment magnitude on beta values for varying equivalent cutoff moment magnitudes. Blue diamonds and lines show the effect where no corner moment is used. Red diamonds and lines show the effect of using 3 different values of equivalent corner magnitudes of, from top to bottom, 6.7, 6.8 and 6.9. Plots on the right show the cumulative number of events greater than the moment shown on the abscissa together with the modeled distributions for the 3 different corner magnitudes.
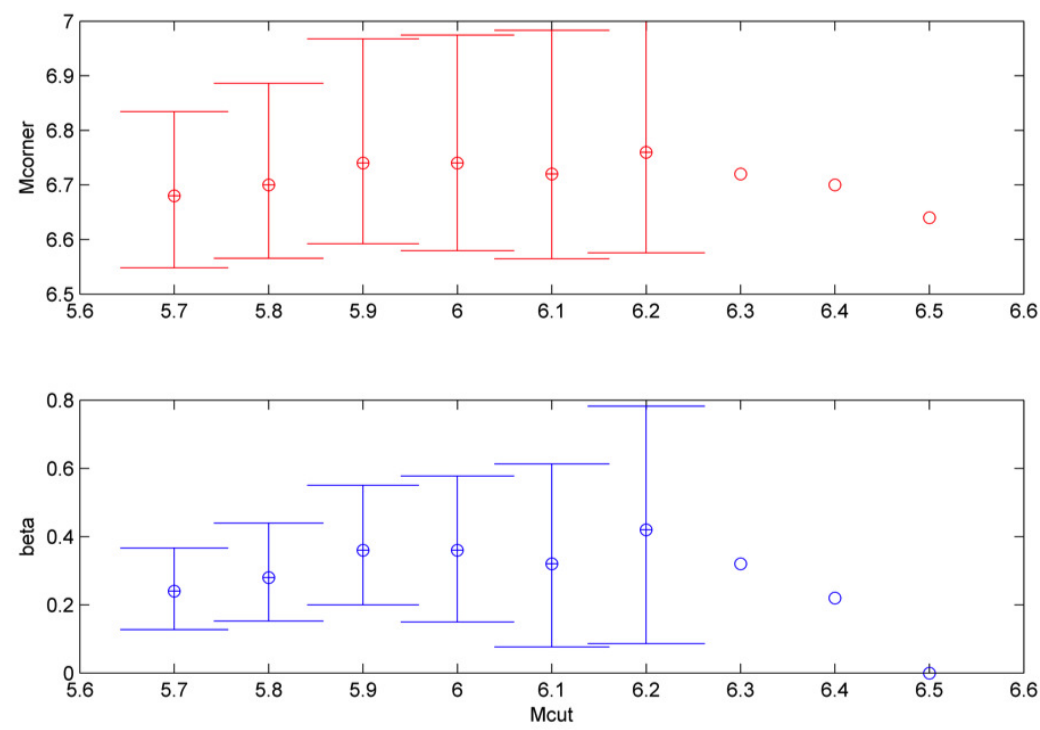

Figure 5.4.2.4b: Plots illustrating the principle of selecting the equivalent moment cutoff magnitude. In both plots the bars represent the $95 \%$ confidence limits on the estimates of the parameters - where there are no bars the estimates are unreliable. In the lower plot, as we move from right to left we are extending the range over which the model is considered stable, and the point at which the beta value starts to decrease indicates the lowest value which can be used for the equivalent moment cutoff magnitude - in this case 5.9. The upper plot confirms that this model is stable for the equivalent moment corner magnitude of approximately 6.74 . 


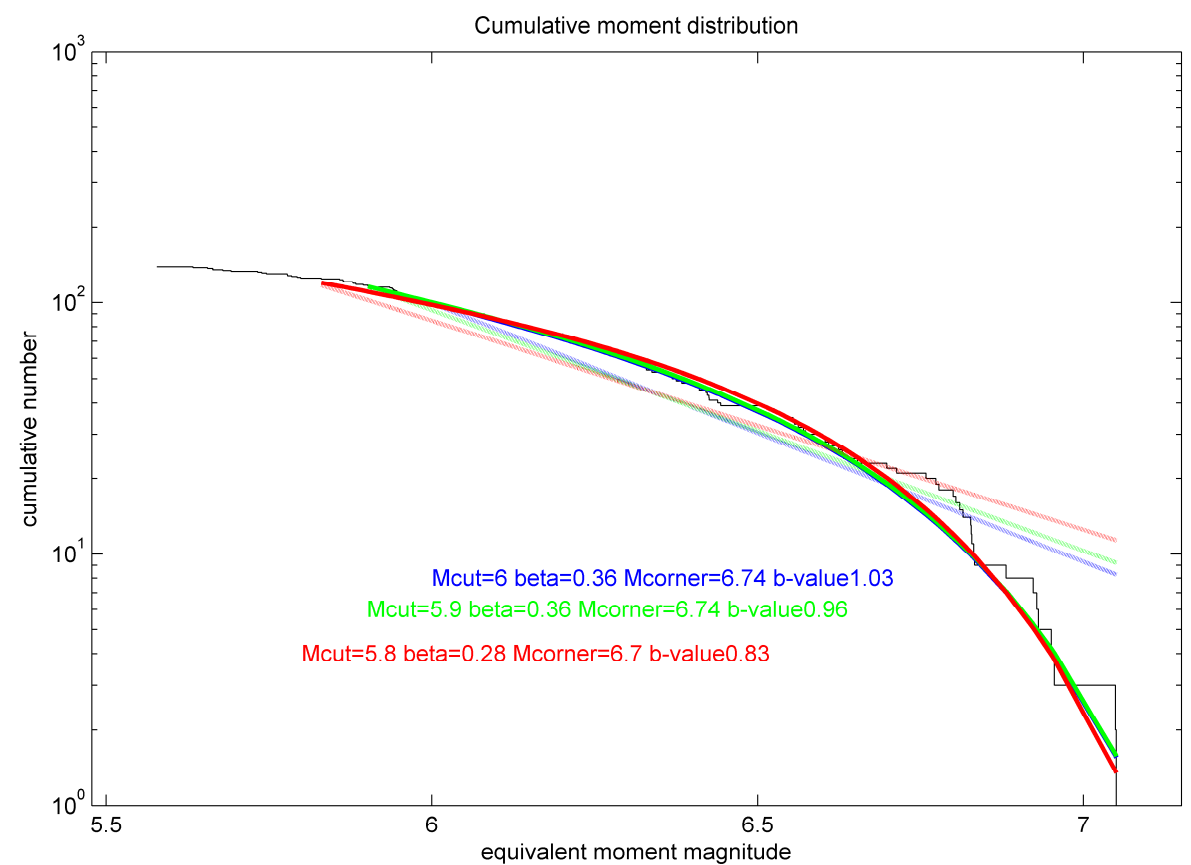

Figure 5.4.2.4c: Three models fitted to the log cumulative number versus equivalent moment magnitude plot. The optimum fit in green is shown bracketed by the two adjacent fits for small changes in equivalent cutoff and corner moment magnitudes. Also shown are the corresponding conventional ordinary linear regression models, illustrating the relatively poor fits to the underlying curvilinear cumulative data, resulting in the ' $b$ ' values not complying with the expected theoretical relationship with the beta values $(\beta=0.667 \mathrm{~b})$

Analyses have been carried out for negative and positive events, and for events in each region. The results are shown in Table 5.4.2.4.

\begin{tabular}{|l|c|c|c|c|c|c|}
\hline Description & No. events & $\mathrm{M}_{\text {cut }}$ & $\mathrm{M}_{\text {corner }}$ & beta & $95 \%$ CI & 'b' value \\
\hline All & 139 & 5.9 & 6.74 & 0.36 & 0.18 & 0.96 \\
\hline Positive & 98 & 5.9 & 6.74 & 0.34 & 0.19 & 0.93 \\
\hline Negative & 41 & 5.9 & 6.70 & 0.44 & nd & 1.06 \\
\hline Northeast & 77 & 5.9 & 6.66 & 0.38 & nd & 1.05 \\
\hline Central & 33 & 5.9 & 6.72 & 0.34 & nd & 0.94 \\
\hline Southwest & 29 & 5.9 & 6.84 & 0.30 & 0.29 & 0.83 \\
\hline
\end{tabular}

Table 5.4.2.4: Moment distribution statistics for events categorized by polarity and by regional location (as defined in 3.2.1). Values 'nd' were deemed unreliable and not determined. 
The results summarised in table 5.4.2.4 indicate no statistically significant difference in the moment distribution parameter beta between any of the groupings. A beta parameter value of 0.36 and $a$ ' $b$ ' value of unity appeared to adequately represent the distribution of moments, within the relatively high uncertainty constraints of this relatively small sample group. The small sample size resulted in a relatively poor fit to the G-R relationship, in that even in the range over which the beta parameter was considered stable, there remained a degree of curvilinearity that resulted in the ratio between beta and ' $b$ ' being closer to 0.36 than the expected theoretical value of 2/3.

The ' $b$ ' value of unity is consistent with the very limited published data on SSEs. Wech et al. [2010] determined a 'b' value of unity based on an analysis of the duration of 93 tremor swarms in northern Cascadia.

\subsubsection{Source total slip amplitudes and rates}

Source total slip amplitudes were calculated as the square root of the sums of the squared dip slip and strike slip components. They showed no significant correlation with other source parameters (i.e. duration, latitude, longitude, and depth), except, of course, with moment, slip area and equivalent moment magnitude.

In section 3.2.3 the speed of surface displacements was noted as being apparently significantly faster in the northeast than in the other regions, although it was commented in 5.2.1 that this could simply be the result of shallower sources. This was re-examined by comparing the source slip speeds (total source slip divided by event duration) in each region. Fig. 5.4.2.5 indicates that whilst the range of source slip speeds in the southwest region was much greater than elsewhere, the mean values were not significantly different in the main regions. Slip speeds were not significantly correlated with other source parameters. 


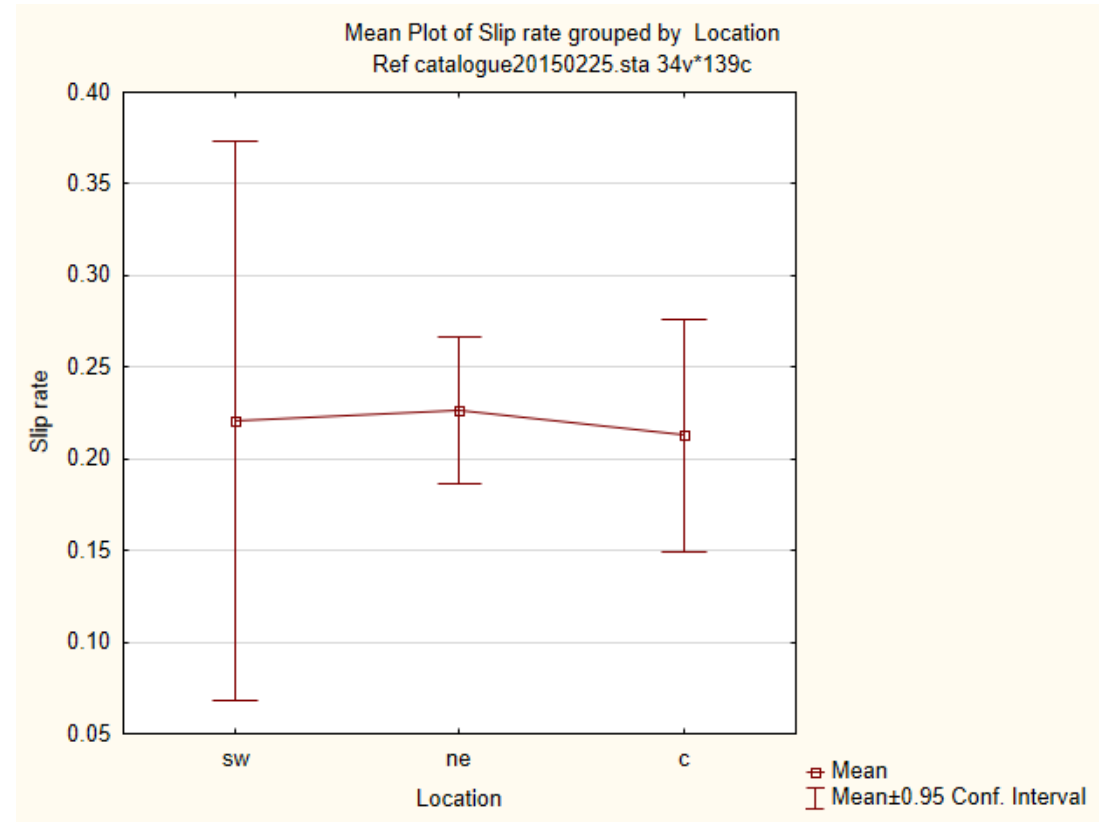

Figure 5.4.2.5: Mean slip speeds (total slip in $\mathrm{cm} /$ duration in days) categorized by source region (sw southwest, ne - northeast, $\mathrm{c}-$ central as defined in 3.2.1). The overlapping 95\% confidence intervals show that there is no significant difference in mean values for each region.

\subsubsection{Differences between events in northeast and southwest of North Island}

With respect to seismic moment distributions, or slip speeds, we have no evidence to indicate any difference in characteristics between events in the northeast and the southwest of the North Island.

\subsection{Effects of subsurface layering}

cGPS sites WGTN (Wellington Airport) and WGTT (Wellington Tide Gauge, situated at The Museum of New Zealand TePapa Tongarewa) are situated within $5 \mathrm{~km}$ of each other, and over $50 \mathrm{~km}$ from epicentres of SSEs located off Kapiti-Marlborough (or the Cook Strait and Seddon earthquakes that are the sources of surface events 144 and 145). The antenna at WGTN is mounted on a concrete column founded on greywacke outcrop $^{4}$, which is part of the Miramar-Mt Crawford structure [Begg and Mazengarb, 1996; Grant-Taylor et al., 1974; Lewis and Mildenhall, 1985; Pillans and Huber,

\footnotetext{
${ }^{4}$ cGPS site details from: https://magma.geonet.org.nz/delta/app
} 
1992]. In contrast, the WGTT antenna is located $23 \mathrm{~m}^{\underline{5}}$ above MSL on the roof of Te Papa, mounted on a pole fixed to the main shear wall, which strikes approximately NE. Te Papa is a base-isolated concrete structure with $3 \mathrm{~m}$-wide strip footings, founded on dynamically-compacted fill, which comprises the first approximately $8 \mathrm{~m}$ of up to 137 $\mathrm{m}$ of reclamation soils and poorly compacted sediments, over greywacke basement [Connolly and Kelly, 1997; Murashev and Palmer, 1998; Semmens et al., 2010a; Semmens et al., 2010b; J H Wood and Martin, 1995]. As part of the site investigation for the building, a strong-motion accelerograph was installed and 20 earthquakes were recorded over the 3-year period from March 1989. This showed typical peak amplifications of the 5\% damped acceleration response spectra between the reference NZSO rock site and the Museum site of between 3 and 4. It is therefore reasonable to expect that, if the results of the layering models have relevance, displacements measured at WGTT due to regional SSEs would be greater than at WGTN.

Over the 14-year period covered by this project, 10 events were detected at WGTN and 14 at WGTT. Two of these, events 144 and 145, were the result of earthquake sequences at Cook Strait and Seddon. Appendix 16 compares the magnitudes of the displacements, and suggests that WGTT experienced approximately 20 to $30 \%$ greater displacements on average than WGTN in the E direction, but approximately $70 \%$ less in the $\mathrm{U}$ direction.

Whilst increased E-component amplitude at WGTT might be expected, given the reported increased acceleration response at the site, it is difficult to explain the magnitude of the increase based solely on modeling of the elastic response of the upper softer layer. Using Wang's EDGRM/EDCMP code and the known stratigraphy and elastic properties of the soft layer (which is assumed to be homogenous and of infinite horizontal extent) [Fry et al., 2010; Perrin and Stephenson, 2010], the maximum difference in E component displacement from WGTN (assumed to be founded directly on greywacke basement), based solely on the elastic response, should be less than $5 \%$, with virtually no difference in the U-component.

\footnotetext{
${ }^{5} 43 \mathrm{~m}$ is shown as the elevation of WGTT in https://magma.geonet.org.nz/delta/app, but the building is located at sea-level and only $22.5 \mathrm{~m}$ high according to Wood \& Martin [1995]. This represents the difference between the geoid (MSL) and WGS84 elevations.
} 
We are therefore left with questions as to whether additional E-component displacement response could be generated by:

- basin edge [Adams and Davis, 2001] or focussing [Iyisan and Khanbabazadeh, 2013] effects, given that the EDGRN/EDCMP model assumes homogenous layers with infinite horizontal extension, which may not be appropriate, since the WGTT sub-site is known to be bounded by buried steep valley sides [Semmens et al., 2010b];

- an elasticity gradient in the soft layer that might produce a greater response than a layer with homogenous elastic properties [Zeng and Benites, 1998];

- cross-coupling between components at the WGTT site [Paolucci, 1999]

- the antenna being mounted on the roof of 23m-high structure. Bevis et al. [2002] indicated that cGPS roof installations could introduce additional displacements due to deformation of the structure;

- the low horizontal stiffness of the Museum's lead-rubber and Teflon slider base isolation system [Islam et al., 2011];

- undocumented deep weathering or discontinuities in the greywacke detected at $137 \mathrm{~m}$ depth in the borehole and assumed to be 'basement'.

The papers referenced in each of the $1^{\text {st }}$ five bullet points discuss effects that have been investigated in relation to dynamic amplification. It is uncertain whether in each case similar effects might be observed in the case of quasi-static strains arising from SSEs. Whilst Zhu and Rivera [2002] have shown that the elasto-dynamic solution tends towards the static elastic solution as $\omega \rightarrow 0$ (although not necessarily at $\omega=0$ ) for a point source in a layered or vertically-heterogeneous medium, it is by no means clear that this applies for the cases mentioned above. It is, however, noted that Benites and Caldwell [2011] have produced transfer function matrices for a number of deep sediment sites around the Wellington and Lower Hutt region that show both amplification and cross coupling values significantly greater than 1 at $0 \mathrm{~Hz}$ (although they state that the values below $0.1 \mathrm{~Hz}$ may not be reliable). Their specific plot for the Te Papa site shows an amplification of 2 at $0 \mathrm{~Hz}$ [Kaiser et al., 2012].

On balance, it is considered that the WGTN-WGTT comparison includes too many unknowns, and therefore does not provide sufficient evidence to validate the use of the simplified EDGRN/EDCMP layer model in this instance. 



\subsection{Conclusions}

A catalogue of 150 discrete events along the Hikurangi margin has been produced covering the 14-year period from the beginning of 2000. The catalogue includes uncertainties for each of the major parameters and is more comprehensive than any other published to date. Of the 150 events, 8 were observed in the Ruapehu area and are considered unlikely to be SSEs on the subduction interface, 2 events could not be adequately constrained in the inversion and therefore are regarded as unreliable, and 3 are directly associated with the 2013 Cook Strait earthquakes. Of the remaining 137 probable SSEs, 41 involve apparent source displacements in the opposite direction to that expected (dubbed 'negative' events).

Many of these 'negative' events were well-resolved in the inversion and unlikely to have been noise. Whilst there is currently not enough evidence for an unambiguous explanation, a number of hypotheses have been discussed, with the most probable of these being that some negative events could represent transient increased friction coupling events. We also conclude that some of the identified SSEs on the margin may not be plate interface events (in the volcanic region, and possibly some smaller negative events), and these require further investigation, as do the differences between the northeast and southwest.

Secular velocities at the cGPS sites have been shown to be significantly affected by the SSEs, especially on the northeast coast from East Cape to Hawkes Bay. The removal of these SSE-derived velocity components significantly changes the pattern of inter-SSE velocities along the margin and raises interesting possibilities for short-term strain field delineation, especially in the northeast where there has been much discussion about the components of rotation.

Preliminary assessment of magnitude scaling relationships indicates that the source events fit within a general $\log \mathrm{M}_{\mathrm{o}} \sim \log \mathrm{T}$ trend suggested by others for SSEs, and the cumulative distribution of seismic moments are not significantly different to a Gutenberg-Richter ' $b$ ' value of unity. However, a more thorough analysis of the probability density distribution indicates an average $\beta$ exponent of $0.36 \pm 0.19$, rather than 0.667 (theoretically equivalent to a unity value of ' $b$ '), leaving further work to be 
carried out as more data becomes available. The discrepancy may be the result of the relatively small number of events and small range of magnitudes resulting from the relatively high detection threshold for GPS, but given that the cGPS network only had full coverage for just over 2 years when this data was acquired, reduced uncertainties should be available in foreseeable future.

The temporal evolution of the non-negative SSEs has been shown to fit an asymmetric reverse sigmoid form, whereas for the negative events a linear ramp form currently appears more appropriate. The theoretical basis proposed by various authors for such a form, based on rate and state friction models, remains contentious and requires further work. From a practical perspective, the 4 parameters required to specify such a model would present difficulties in use in an automated search template, and some alternatives are proposed.

The effects on inversion of stratigraphic layering in the hanging wall have been explored by parametric modeling, but within the time constraints of the project, this work could not be concluded and is ongoing. This aspect was not anticipated when the project was initiated and did not form part of the scope.

With respect to the objectives of exploring potential differences between events in the northeast and southwest of the margin, in the limited time available, no fundamental differences could be found in temporal evolution, source slip rates, or scaling between these two regions, which appeared to form a depth continuum. However, the durations of events in the southwest are generally longer than those in the northeast, and there remains further work to be done to explore segmentation along the boundary in a more thorough statistical manner, especially since there are indications of a low occurrence rate in the spatial evolution of events around the southern Hawkes Bay area.

In addition to updating of the database to cover the period since Feb 2014, further work is required in the following areas:

- Automated search algorithms

- Improved inversion techniques

- Analyses for any effects of stratigraphic layering on inversion results

- Statistical analyses on spatial segmentation and recurrence rates 


\section{References}

Abercrombie, R. E., M. Antolik, K. Felzer, and G. Ekström (2001), The 1994 Java tsunami earthquake: Slip over a subducting seamount, Journal of Geophysical Research, 106(B4), 6595.

Abujiya, M. a. R., M. Riaz, and M. H. Lee (2013), Improving the Performance of Combined Shewhart-Cumulative Sum Control Charts, Quality and Reliability Engineering International, 29(8), 1193-1206.

Adams, B. M., and R. O. Davis (2001), Seismic wave amplification at the edge of a soft layer, in New Zealand Geotechnical Society 2001 Symposium, edited, pp. 197 - 207, Christchurch.

Ansell, J. H., and S. C. Bannister (1996), Shallow morphology of the subducted Pacific Plate along the Hikurangi margin, New Zealand, Physics of the Earth and Planetary Interiors, 93, 3 20.

Approximatrix (2014), Simply Fortran, edited.

Árnadóttir, T., S. Thornley, F. F. Pollitz, and D. J. Darby (1999), Spatial and temporal strain rate variations at the northern Hikurangi margin, New Zealand, Journal of Geophysical Research, 104(B3), 4931.

Audet, P., and R. Bürgmann (2014), Possible control of subduction zone slow-earthquake periodicity by silica enrichment, Nature, 510(7505), 389-392.

Bannister, S., M. Reyners, G. Stuart, and M. Savage (2007), Imaging the Hikurangi subduction zone, New Zealand, using teleseismic receiver functions: crustal fluids above the forearc mantle wedge, Geophysical Journal International, 169(2), 602-616.

Barnes, P. M., G. Lamarche, J. Bialas, S. Henrys, I. Pecher, G. L. Netzeband, J. Greinert, J. J. Mountjoy, K. Pedley, and G. Crutchley (2010), Tectonic and geological framework for gas hydrates and cold seeps on the Hikurangi subduction margin, New Zealand, Marine Geology, 272(1-4), 26-48.

Barrett, R. (2014), Investigating the evolution with time of slow slip events along the Hikurangi Trench, New ZealandRep., 42 pp, Victoria University of Wellington, Wellington.

Bartlow, N. M., L. M. Wallace, J. Beavan, S. Bannister, and P. Segall (2014), Time-dependent modeling of slow slip events and associated seismicity and tremor at the Hikurangi subduction zone, New Zealand, Journal of Geophysical Research: Solid Earth, n/a-n/a.

Bassett, D. G., R. Sutherland, and S. Henrys (2014), Slow wavespeeds and fluid overpressure in a region of shallow geodetic locking and slow slip, Hikurangi subduction margin, New Zealand, Earth and Planetary Science Letters, 389, 1-13.

Bassett, D. G., R. Sutherland, S. Henrys, T. Stern, M. Scherwath, A. Benson, S. Toulmin, and M. Henderson (2010), Three-dimensional velocity structure of the northern Hikurangi margin, Raukumara, New Zealand: Implications for the growth of continental crust by subduction erosion and tectonic underplating, Geochemistry, Geophysics, Geosystems, 11(10), 24.

Beanland, S., and J. Haines (1988), The kinematics of active deformation in the North Island, New Zealand, determined from geological strain rates, N. Z. J. Geol. Geophys., 41(1). 
Beavan, J. (2005), Noise properties of continuous GPS data from concrete pillar geodetic monuments in New Zealand and comparison with data from U.S. deep drilled braced monuments, Journal of Geophysical Research, 110(B8).

Beavan, J., and J. Haines (2001), Contemporary horizontal velocity and strain rate fields of the Pacific-Australian plate boundary zone through New Zealand, Journal of Geophysical Research, 106(B1), 741.

Beavan, J., and D. J. Darby (2005), Fault slip in the 1855 Wairarapa Earthquake based on new and reassessed vertical motion observations: Did slip occur on the subduction interface?, in The 1855 Wairarapa Earthquake Symposium, edited by R. L. John Townend, and Andrew Jones, pp. 31-41, Greater Wellington Regional Council, Museum of New Zealand Te Papa Tongarewa, Wellington.

Beavan, J., and L. M. Wallace (2008), It's Our Fault - Wellington geodetic studies task completion report - Fault coupling results from inversion of new and reprocessed GPS campaign data from the Wellington region, Consultancy report Rep., 12 pp, GNS Science.

Beavan, J., R. McCaffrey, M. E. Reyners, and L. M. Wallace (2008), Slow slip events and small earthquake clustering - implications for the locked region of the shallow Hikurangi subduction zoneRep., 21 pp, GNS Science.

Beavan, J., P. Tregoning, M. Bevis, T. Kato, and C. Meertens (2002), Motion and rigidity of the Pacific plate and implications for plate boundary deformation, J. Geophys. Res., 107(B10).

Beavan, J., L. M. Wallace, A. Douglas, H. Fletcher, and J. Townend (2007), Slow-slip events on the Hikurangi subduction interface, New Zealand, in IAG symposium, edited by P. Tregoning and C. Rizos, p. 7, Cairns, Australia.

Begg, J. G., and C. Mazengarb (1996), Geology of the Wellington Area scale 1:50,000, Sheets R27, R28 and part Q27, Scale 21:50,000, IGNS, Lower Hutt.

Bell, R., C. Holden, W. L. Power, X. Wang, and G. Downes (2014), Hikurangi margin tsunami earthquake generated by slow seismic rupture over a subducted seamount, Earth and Planetary Science Letters, 397, 1-9.

Bell, R., R. Sutherland, D. H. N. Barker, S. Henrys, S. Bannister, L. M. Wallace, and J. Beavan (2010), Seismic reflection character of the Hikurangi subduction interface, New Zealand, in the region of repeated Gisborne slow slip events, Geophysical Journal International, 180(1), 34-48.

Benites, R., and T. G. Caldwell (2011), A Ground-Motion Transfer Function Matrix between Two Nearby Rock and Soil Sites: A System Identification Problem, Bulletin of the Seismological Society of America, 101(1), 222-234.

Bennett, R. A. (2008), Instantaneous deformation from continuous GPS: contributions from quasi-periodic loads, Geophysical Journal International, 174(3), 1052-1064.

Berryman, K., M. Marden, A. Palmer, and N. Litchfield (2009), Holocene rupture of the Repongaere fault, Gisborne: Implications for Raukumara Peninsula deformation and impact on the Waipaoa Sedimentary System, New Zealand Journal of Geology and Geophysics, 52(4), 335-347.

Besana, G. M., and M. Ando (2005), The central Philippine Fault zone: Location of great earthquakes, slow events and creep activity, Earth, Planets and Space, 57, 987-994.

Bevis, M., W. Scherer, and M. Merrifield (2002), Technical issues and recommendations related to the installation of cGPS stations at tide gauges, Marine Geodesy, 25, 87 - 99. 
Birch, C. P. D. (1999), A new generalised logistic sigmoid growth equation compared with the Richards Growth Equation, Annals of Botany, 83, 713 - 723.

Bland, J. M., and D. G. Altman (1999), Measuring agreement in method comparison studies, Stat. Methods in Med. Res., 8, 135 - 160.

Bock, Y., R. M. Nikolaidis, P. J. de Jonge, and M. Bevis (2000), Instantaneous geodetic positioning at medium distances with the Global Positioning System, Journal of Geophysical Research, 105(B12), 28223.

Borsa, A. A., D. C. Agnew, and D. R. Cayan (2014), Ongoing drought-induced uplift in the western United States, Science, 345(6204).

Bosy, J. (2013), Global, Regional and National Geodetic Reference Frames for Geodesy and Geodynamics, Pure and Applied Geophysics, 171(6), 783-808.

Boulton, C., B. M. Carpenter, V. Toy, and C. Marone (2012), Physical properties of surface outcrop cataclastic fault rocks, Alpine Fault, New Zealand, Geochemistry, Geophysics, Geosystems, 13(1), n/a-n/a.

Brace, R. A. (1977), Fitting straight lines to experimental data, Am. J. Physiol., 233(3), R94 R99.

Brudzinski, M. R., and R. M. Allen (2007), Segmentation in episodic tremor and slip all along Cascadia, Geology, 35(10), 907.

Bullen, K. E. (1963), 10.4.3.1 The method of uniform reduction, in Introduction to the theory of seismology, edited, pp. 162 - 163, Cambridge University Press, Cambridge.

Bürgmann, R., M. G. Kogan, V. E. Levin, C. H. Scholz, R. W. King, and G. M. Steblov (2001), Rapid aseismic moment release following the 5 December, 1997 Kronotsky, Kamchatka, Earthquake, Geophysical Research Letters, 28(7), 1331-1334.

CAANZ (2006), Mountain Flying, edited by CAA, p. 32, Civil Aviation Authority of New Zealand.

Calais, E. (2010), GPS noise models, edited, p. 30, Purdue University.

Campione, M., and G. C. Capitani (2013), Subduction-zone earthquakes complexity related to frictional anisotropy in antigorite, Nature Geoscience, 18 Aug 2013, doi: 10.1038/ngeo1905.

Cashman, S. M., and H. M. Kelsey (1990), Forearc uplift and extension, southern Hawkes Bay, New Zealand: Mid-Pleistocene to present, Tectonics, 9(1), 23 - 44.

Cashman, S. M., H. M. Kelsey, C. F. Erdman, H. N. C. Cutten, and K. R. Berryman (1992), Strain partitioning between structural domains in the forearc of the Hikurangi subduction zone, New Zealand, Tectonics, 11(2), 242 - 257.

Cattin, R., H. Lyon-Caen, and J. Chéry (1997), Quantification of interplate coupling in subduction zones and forearc topography, Geophysical Research Letters, 24(13), 1563-1566.

Cattin, R., P. Briole, H. Lyon-Caen, P. Bernard, and P. Pinettes (1999), Effects of superficial layers on coseismic displacemnts for a dip-slip fault and geophysical implications, Geophysical Journal International, 137, 140 - 158.

Cavalié, O., E. Pathier, M. Radiguet, M. Vergnolle, N. Cotte, A. Walpersdorf, V. Kostoglodov, and F. Cotton (2013), Slow slip event in the Mexican subduction zone: Evidence of shallower 
slip in the Guerrero seismic gap for the 2006 event revealed by the joint inversion of InSAR and GPS data, Earth and Planetary Science Letters, 367, 52-60.

Chang, E. T. Y., and B. F. Chao (2014), Analysis of coseismic deformation using EOF method on dense, continuous GPS data in Taiwan, Tectonophysics, 637, 106-115.

Chao, T.-K. K. (2012), Global search of triggered non-volcanic tremor, 156 pp, Georgia Institute of Technology.

Chen, W., S. Ni, S. Wei, Z. Wang, and J. Xie (2011), Effects of sedimentary layer on earthquake source modeling from geodetic inversion, Earthquake Science, 24(2), 221-227.

Ching, K.-E., Y.-C. Chen, and R.-J. Rau (2014), First detection of the 2009 slow slip event under northeast Taiwan at the Ryukyu subduction system from continuous GPS data, in EGU, edited, Geophysical Reserach Abstracts.

Clark, K. J., B. W. Hayward, U. A. Cochran, H. R. Grenfell, E. Hemphill-Haley, D. C. Mildenhall, M. A. Hemphill-Haley, and L. M. Wallace (2011), Investigating subduction earthquake geology along the southern Hikurangi margin using palaeoenvironmental histories of intertidal inlets, New Zealand Journal of Geology and Geophysics, 54(3), 255-271.

Clarke, A. J., and S. Van Gorder (2013), On Fitting a Straight Line to Data when the "Noise" in Both Variables Is Unknown*, Journal of Atmospheric and Oceanic Technology, 30(1), 151-158.

Colella, H. V., J. H. Dieterich, K. Richards-Dinger, and A. M. Rubin (2012), Complex characteristics of slow slip events in subduction zones reproduced in multi-cycle simulations, Geophysical Research Letters, 39(20), L20312 - L20317.

Connolly, P., and T. Kelly (1997), Museum of New Zealand : Te Papa Tongarewa Establishment of Structural Design Parameters, in IPENZ annual conference, edited, pp. 73 78.

Cox, M. G., and B. R. L. Siebert (2006), The use of a Monte Carlo method for evaluating uncertainty and expanded uncertainty, Metrologia, 43(4), S178-S188.

Crowell, B. W. (2013), Using GPS to rapidly detect and model earthquakes and transient deformation events, 148 pp, University of California, San Diego.

Crowell, B. W., Y. Bock, and D. Melgar (2012), Real-time inversion of GPS data for finite fault modeling and rapid hazard assessment, Geophysical Research Letters, 39(9).

Darby, D. J., and C. M. Meertens (1995), Terrestrial and GPS measurements of deformation across the Taupo back arc and Hikurangi forearc regions in New Zealand, Journal of Geophysical Research, 100(B5), 8221.

Darby, D. J., and J. Beavan (2001), Evidence from GPS measurements for contemporary interplate coupling on the southern Hikurangi subduction thrust and for partitioning of strain in the upper plate, Journal of Geophysical Research, 106(B12), 30881.

Davey, F. J., and J. Ristau (2011), Fore-arc mantle wedge seismicity under northeast New Zealand, Tectonophysics, 509(3-4), 272-279.

Davis, E., K. Becker, K. Wang, K. Obara, Y. Ito, and M. Kinoshita (2006), A discrete episode of seismic and aseismic deformation of the Nankai trough subduction zone accretionary prism and incoming Philippine Sea plate, Earth and Planetary Science Letters, 242(1-2), 73-84. 
Davis, J. L., B. P. Wernicke, and M. E. Tamisiea (2012), On seasonal signals in geodetic time series, Journal of Geophysical Research, 117(B1).

Delahaye, E. J., J. Townend, M. E. Reyners, and G. Rogers (2009), Microseismicity but no tremor accompanying slow slip in the Hikurangi subduction zone, New Zealand, Earth and Planetary Science Letters, 277(1-2), 21-28.

den Hartog, S. A. M., and C. J. Spiers (2014), A microphysical model for fault gouge friction applied to subduction megathrusts, Journal of Geophysical Research: Solid Earth, 19.

Dermanis, A., and C. Kotsakis (2005), Estimating crustal deformation parameters from geodetic data: Review of existing methodologies, open problems and new challenges, paper presented at Geodetic Deformation Monitoring: From Geophysical to Engineering Roles, Springer Berlin Heidelberg, IAG Symposium Jaén, Spain, March 17-19, 2005.

Dieterich, J. H. (1978), Time-dependent friction and the mechanics of stick-slip, Pageoph., 116, 790 - 806.

Dimitrova, L. L., A. J. Haines, L. M. Wallace, and C. A. Williams (2014), High resolution view of active tectonic deformation in the North Island, New Zealand, edited, submitted manuscript.

Doser, D. I., and T. H. Webb (2003), Source parameters of large historical (1917 - 1961) earthquakes, North Island, New Zealand, Geophys. J. Int., 2003(152), 795 - 832.

Douglas, A., J. Beavan, L. M. Wallace, and J. Townend (2005), Slow slip on the northern Hikurangi subduction interface, New Zealand, Geophysical Research Letters, 32(16).

Downes, G., D. Dowrick, E. G. C. Smith, and K. Berryman (1997), The 1934 Pahiatua earthquake sequence: analysis of observational and instrumental dataRep. 0018, EQC, Wellington.

Eberhart-Phillips, D., and W. D. Stuart (1992), Material heterogeneity simplifies the picture Loma Prieta, Bulletin of Seismological Society of America, 82(4), 1964 - 1968.

Eberhart-Phillips, D., and M. Reyners (2012), Imaging the Hikurangi Plate interface region, with improved local-earthquake tomography, Geophysical Journal International, 190(2), 1221-1242.

Eberhart-Phillips, D., M. Reyners, M. Chadwick, and G. Stuart (2008), Three-dimensional attenuation structure of the Hikurangi subduction zone in the central North Island, New Zealand, Geophysical Journal International, 174(1), 418-434.

Eberhart-Phillips, D., M. Reyners, S. Bannister, M. Chadwick, and S. Ellis (2010), Establishing a Versatile 3-D Seismic Velocity Model for New Zealand, Seismological Research Letters, 81(6), 992-1000.

Ewig, E. (2009), Lithospheric shortening and ductile deformation in a back-arc setting: South Wanganui Basin, New Zealand, Victoria University of wellington, Wellington.

Fagereng, A. (2011), Wedge geometry, mechanical strength, and interseismic coupling of the Hikurangi subduction thrust, New Zealand, Tectonophysics, 507(1-4), 26-30.

Fagereng, A., and S. Ellis (2009), On factors controlling the depth of interseismic coupling on the Hikurangi subduction interface, New Zealand, Earth and Planetary Science Letters, 278(12), 120-130. 
Feng, L., A. V. Newman, M. Protti, V. González, Y. Jiang, and T. H. Dixon (2012), Active deformation near the Nicoya Peninsula, northwestern Costa Rica, between 1996 and 2010: Interseismic megathrust coupling, Journal of Geophysical Research, 117(B6).

Foster, J. H., A. R. Lowry, and B. A. Brooks (2013), Fault frictional parameters and material properties revealed by slow slip events at Kilauea volcano, Hawai $i$, Geophysical Research Letters, 40(23), 6059-6063.

Fry, B., R. Benites, W. R. Stephenson, and P. Barker (2010), It's our fault - Seismic instrumentation and inversion for physical parameters of Wellington and the Hutt ValleyRep. 2010/18, 47 pp, GNS Science, Wellington.

Fry, B., K. Chao, S. Bannister, Z. Peng, and L. M. Wallace (2011), Deep tremor in New Zealand triggered by the 2010 Mw8.8 Chile earthquake, Geophysical Research Letters, 38(15), L15306 L15311.

Fu, Y., and J. T. Freymueller (2013), Repeated large Slow Slip Events at the southcentral Alaska subduction zone, Earth and Planetary Science Letters, 375, 303-311.

Fukuda, J., A. Kato, K. Obara, S. Miura, and T. Kato (2014), Imaging of the early acceleration phase of the 2013-2014 Boso slow slip event, Geophysical Research Letters, 41(21), 74937500.

Furlong, K. P., and P. J. J. Kamp (2009), The lithospheric geodynamics of plate boundary transpression in New Zealand: Initiating and emplacing subduction along the Hikurangi margin, and the tectonic evolution of the Alpine Fault system, Tectonophysics, 474(3-4), 449-462.

Gao, H., D. A. Schmidt, and R. J. Weldon (2012), Scaling Relationships of Source Parameters for Slow Slip Events, Bulletin of the Seismological Society of America, 102(1), 352-360.

Gao, X., and K. Wang (2014), Strength of stick-slip and creeping subduction megathrusts from heat flow observations, Science, 345(6200), 1038-1041.

Geonet (2013), GPS processing notes.

GNS Sedimentary Basins, edited, p. New Zealand sedimentary basin depths, GNS, Wellington.

Gökalp, E., O. Güngör, and Y. Boz (2008), Evaluation of Different Outlier Detection Methods for GPS Networks, Sensors, 8(11), 7344-7358.

Google (2013), GoogleEarth, edited.

Grandin, R., et al. (2009), September 2005 Manda Hararo-Dabbahu rifting event, Afar (Ethiopia): Constraints provided by geodetic data, Journal of Geophysical Research, 114(B8).

Grant-Taylor, T. L., R. D. Adams, W. R. Stephenson, J. D. G. Milne, R. D. Northey, and T. Hatherton (1974), Microzoning for earthquake effects in Wellington, N. Z.Rep. ISSN 0077-916X, $62 \mathrm{pp}, \mathrm{DSIR}$, Wellington.

Gregorius, T., and G. Blewitt (1998), The effect of weather fronts on GPS measurements, in GPS world, edited, pp. 52 - 60.

Gutenberg, B., and C. F. Richter (1944), Frequency of earthquakes in California, Bull. Seism. Soc. Am., 34(4), 185 - 188.

Hall, L. C. (2013), Seismic structure beneath the Wellington region from receiver functions, 201 $\mathrm{pp}$, Victoria University of Wellington, Wellington. 
Hamling, I. J., E. D'Anastasio, L. M. Wallace, S. Ellis, M. Motagh, S. Samsonov, N. Palmer, and S. Hreinsdóttir (2014), Crustal deformation and stress transfer during a propagating earthquake sequence: The 2013 Cook Strait sequence, central New Zealand, Journal of Geophysical Research: Solid Earth, 119(7), 6080-6092.

Hayes, G. P., D. J. Wald, and R. L. Johnson (2012), Slab1.0: A three-dimensional model of global subduction zone geometries, Journal of Geophysical Research, 117(B1).

Hearn, E. H., and R. Burgmann (2005), The Effect of Elastic Layering on Inversions of GPS Data for Coseismic Slip and Resulting Stress Changes: Strike-Slip Earthquakes, Bulletin of the Seismological Society of America, 95(5), 1637-1653.

Heise, W., T. G. Caldwell, G. J. Hill, S. L. Bennie, E. Wallin, and E. A. Bertrand (2012), Magnetotelluric imaging of fluid processes at the subduction interface of the Hikurangi margin, New Zealand, Geophysical Research Letters, 39(4).

Henrys, S., et al. (2013), SAHKE geophysical transect reveals crustal and subduction zone structure at the southern Hikurangi margin, New Zealand, Geochemistry, Geophysics, Geosystems, 14(7), 2063-2083.

Heuret, A., S. Lallemand, F. Funiciello, C. Piromallo, and C. Faccenna (2011), Physical characteristics of subduction interface type seismogenic zones revisited, Geochemistry, Geophysics, Geosystems, 12(1), 26.

Holtkamp, S. G., and M. R. Brudzinski (2011), Earthquake swarms in circum-Pacific subduction zones, Earth and Planetary Science Letters, 305(1-2), 215-225.

Holtkamp, S. G., M. E. Pritchard, and R. B. Lohman (2011), Earthquake swarms in South America, Geophysical Journal International, 187(1), 128-146.

Höök, M., J. Li, N. Oba, and S. Snowden (2011), Descriptive and Predictive Growth Curves in Energy System Analysis, Natural Resources Research, 20(2), 103-116.

Hreinsdóttir, S., J. T. Freymueller, R. Bürgmann, and J. Mitchell (2006), Coseismic deformation of the 2002 Denali Fault earthquake: Insights from GPS measurements, Journal of Geophysical Research, 111(B3).

Huang, M. H., D. Dreger, R. Burgmann, S. H. Yoo, and M. Hashimoto (2013), Joint inversion of seismic and geodetic data for the source of the 2010 March 4, Mw 6.3 Jia-Shian, SW Taiwan, earthquake, Geophysical Journal International, 193(3), 1608-1626.

Hyndman, R. D. (2007), The seismogenic zone of subduction thrust faults: What we know and don't know,, in The Seismogenic Zone of Subduction Thrust Faults, edited by T. H. Dixon and J. C. Moore, pp. 15 - 40, Columbia University Press, New York.

Ide, S. (2012), Variety and spatial heterogeneity of tectonic tremor worldwide, Journal of Geophysical Research, 117(B3).

Ide, S., G. C. Beroza, D. R. Shelly, and T. Uchide (2007), A scaling law for slow earthquakes, Nature, 447(7140), 76-79.

Ide, S., K. Imanishi, Y. Yoshida, G. C. Beroza, and D. R. Shelly (2008), Bridging the gap between seismically and geodetically detected slow earthquakes, Geophysical Research Letters, 35(10).

IGS (2013), IGS Products. 
Ikari, M. J., and D. M. Saffer (2011), Comparison of frictional strength and velocity dependence between fault zones in the Nankai accretionary complex, Geochemistry, Geophysics, Geosystems, 12(4), n/a-n/a.

Islam, A. B. M. S., M. Jameel, and M. Z. Jumaat (2011), Seismic isolation in buildings to be a practical reality: Behavior of structure and installation technique, Jnl. Eng \& Technol. Res., 3(4), $99-117$.

lyisan, R., and $\mathrm{H}$. Khanbabazadeh (2013), A numerical study on the basin edge effect on soil amplification, Bulletin of Earthquake Engineering, 11(5), 1305-1323.

Jeffreys, H. (1932), An alternative to the rejection of observations, Proc. Royal Soc. London Series A, 137(831), 78-87.

Jeffreys, H. (1961), Ch. 4 Approximate methods and simplifications, in Theory of Probability, edited, pp. 212 - 216, Clarendon Press, Oxford.

Ji, C., D. J. Wald, and D. V. Helmberger (2002), Source description of 1999 Hector mine, California, Earthquake, Part 1: Wavelet domain inversion theory and resolution analysis, Bulletin of Seismological Society of America, 92(4), 1192 - 1207.

Jiang, Y., S. Wdowinski, T. H. Dixon, M. Hackl, M. Protti, and V. Gonzalez (2012), Slow slip events in Costa Rica detected by continuous GPS observations, 2002-2011, Geochemistry, Geophysics, Geosystems, 13(4), n/a-n/a.

Kagan, Y. Y. (1991), Seismic moment distribution, Geophys. J. Int., 106, 123 - 134.

Kagan, Y. Y. (2002), Seismic moment distribution revisited: 1. Statistical results, Geophys. J. Int., $148,520-541$.

Kaiser, A., C. Holden, J. Zhao, G. McVerry, and R. Benites (2012), It's Our Fault: Ground motion modelling of local site effects in the Wellington regionRep. 2012/172, 41 pp, GNS Science, Wellington.

Kanamori, H. (1977), The energy release in great earthquakes, Journal of Geophysical Research, 82(20), 2981-2987.

Kanamori, H., and J. J. Cipar (1974), Focal process of the great Chilean earthquake May 22, 1960, Physics of the Earth and Planetary Interiors, 9, 128 - 136.

Kanamori, H., and D. L. Anderson (1975), Theoretical basis of some empirical relations in seismology, Bull. Seism. Soc. Am., 65(5), 1073 - 1095.

Kaneko, Y., J.-P. Avouac, and N. Lapusta (2010), Towards inferring earthquake patterns from geodetic observations of interseismic coupling, Nature Geoscience, 3(5), 363-369.

Kaproth, B. M., and C. Marone (2013), Slow earthquakes, preseismic velocity changes, and the origin of slow frictional stick-slip, Science, 341(6151), 1229-1232.

Karkach, A. (2006), Trajectories and models of individual growth, Demographic Research, 15, 347-400.

Katayama, I., M. Iwata, K. Okazaki, and K. Hirauchi (2013), Slow earthquakes associated with fault healing on a serpentinized plate interface, Scientific reports, 3, 1784. 
Kim, M. J., S. Y. Schwartz, and S. Bannister (2011), Non-volcanic tremor associated with the March 2010 Gisborne slow slip event at the Hikurangi subduction margin, New Zealand, Geophysical Research Letters, 38(14), L14301 - L14305.

King, D. N. T., J. Goff, and A. Skipper (2010), Māori environmental knowledge and natural hazards in Aotearoa-New Zealand, Journal of the Royal Society of New Zealand, 37(2), 59-73.

King, M. A., and S. D. P. Williams (2009), Apparent stability of GPS monumentation from shortbaseline time series, Journal of Geophysical Research, 114(B10).

Kodaira, S., T. lidaka, A. Kato, P. Jin-Oh, T. Iwasaki, and Y. Kaneda (2004), High pore fluid pressure may cause silent slip in the Nankai Trough, Science, 304(5675).

Kreemer, C., G. Blewitt, and E. C. Klein (2014), A geodetic plate motion and Global Strain Rate Model, Geochemistry, Geophysics, Geosystems, 15(10), 3849-3889.

Kucharavy, D., and R. De Guio (2011), Application of S-shaped curves, Procedia Engineering, 9, 559-572.

Lamb, S. H. (1988), Tectonic rotations about vertical axes during the last $4 \mathrm{Ma}$ in part of the New Zealand plate boundary zone, Journal of Structural Geology, 10(8), 875 - 893.

Lamb, S. H. (2011), Cenozoic tectonic evolution of the New Zealand plate-boundary zone: A paleomagnetic perspective, Tectonophysics, 509(3-4), 135-164.

Lamb, S. H., and E. G. C. Smith (2013), The nature of the plate interface and driving force of interseismic deformation in the New Zealand plate-boundary zone, revealed by the continuous GPS velocity field, Journal of Geophysical Research: Solid Earth, 118(6), 3160-3189.

Larson, K. M., A. R. Lowry, V. Kostoglodov, W. Hutton, O. Sanchez, K. Hudnut, and G. Suarez (2004), Crustal deformation measurements in Guerrero, Mexico, Journal of Geophysical Research, 109(B4).

Lewis, K. B., and D. C. Mildenhall (1985), The late Quaternary seismic, sedimentary and palynological stratigraphy beneath Evans Bay, Wellington Harbour, New Zealand Journal of Geology and Geophysics, 28(1), 129-152.

Lidberg, M., C. Eksrom, and J. M. Johansson (2007), Site-dependent effects in high accuracy applications of GNSSRep., EUREF.

Limpert, E., W. A. Stahel, and M. Abbt (2001), Log-normal distributions across the sciences, BioScience, 51(5), 341 - 352.

LINZ (2000), New Zealand Transverse Mercator 2000, edited.

Litchfield, N. J., et al. (2014), A model of active faulting in New Zealand, New Zealand Journal of Geology and Geophysics, 57(1), 32-56.

Liu, C., A. T. Linde, and I. S. Sacks (2009), Slow earthquakes triggered by typhoons, Nature, 459(7248), 833-836.

Liu, P. (2006), Kinematic Inversion of the 2004 M 6.0 Parkfield Earthquake Including an Approximation to Site Effects, Bulletin of the Seismological Society of America, 96(4B), S143S158. 
Liu, P., and R. J. Archuleta (2004), A new nonlinear finite fault inversion with three-dimensional Green's functions: Application to the 1989 Loma Prieta, California, earthquake, Journal of Geophysical Research, 109(B2).

Liu, Y. (2007), Physical basis of aseismic deformation transients in subduction zones, 206 pp, Harvard, Cambridge, Massachusetts.

Liu, Y. (2014), Source scaling relations and along-strike segmentation of slow slip events in a 3D subduction fault model, Journal of Geophysical Research: Solid Earth, 119(8), 6512-6533.

Liu, Y., and J. R. Rice (2005), Aseismic slip transients emerge spontaneously in threedimensional rate and state modeling of subduction earthquake sequences, Journal of Geophysical Research, 110(B8).

Liu, Y., and J. R. Rice (2007), Spontaneous and triggered aseismic deformation transients in a subduction fault model, Journal of Geophysical Research, 112(B9).

Liu, Y., and J. R. Rice (2009), Slow slip predictions based on granite and gabbro friction data compared to GPS measurements in northern Cascadia, Journal of Geophysical Research, 114(B9).

Liu, Y., and A. M. Rubin (2010), Role of fault gouge dilatancy on aseismic deformation transients, Journal of Geophysical Research, 115(B10).

Lowry, A. R. (2015), Pers. Comm., edited.

Lowry, A. R., M. W. Hamburger, C. M. Meertens, and E. G. Ramos (2001a), GPS monitoring of crustal deformation at Taal volcano, Philippines, J. Volcanology and geothermal research, 105, $35-47$.

Lowry, A. R., K. M. Larson, V. Kostoglodov, and R. Bilham (2001b), Transient fault slip in Guerrero, southern Mexico, Geophys. Res. Lett., 28, 3753-3756.

Ma, Y., R. Wang, and H. Zhou (2012), A note on the equivalence of three major propagator algorithms for computational stability and efficiency, Earthquake Science, 25(1), 55-64.

Malik, H. J. (2006), Logistic distribution, in Encylopedia of Statistical Sciences, edited, p. 5, John Wiley.

Mao, A., C. G. A. Harrison, and T. H. Dixon (1999), Noise in GPS coordinate time series, Journal of Geophysical Research, 104(B2), 2797.

Marjetič, A., T. Ambrožič, G. Turk, O. Sterle, and B. Stopar (2010), Statistical properties of strain and rotation tensors in geodetic network, Jnl. Surveying Engineering, 136(3).

Marone, C. (1998), Laboratory-derived friction laws and their application to seismic faulting, Anna. Rev. Earth Planet Sci., 26, 643-696.

Masinha, L., and D. E. Smylie (1971), The displacement fields of inclined faults, Bull. Seism. Soc. Am., 61(5), 1433 - 1440.

Masterlark, T. (2003), Finite element model predictions of static deformation from dislocation sources in a subduction zone: Sensitivities to homogeneous, isotropic, Poisson-solid, and halfspace assumptions, Journal of Geophysical Research, 108(B11).

Mathworks (2014), MATLAB, edited. 
Matsuzawa, T., H. Hirose, B. Shibazaki, and K. Obara (2010), Modeling short- and long-term slow slip events in the seismic cycles of large subduction earthquakes, Journal of Geophysical Research, 115(B12).

McCaffrey, R. (1997), Statistical significance of the seismic coupling coefficient, Bulletin of Seismological Society of America, 87(4), 1069 - 1073.

McCaffrey, R. (2007), DEFNODE User's Manual Version 2007.10.25, edited, p. Elastic block modelling program used by GNS for GPS data inversion, http://web.pdx.edu/\%7Emccaf/www/defnode/defnode 071025.html.

McCaffrey, R. (2008), Global frequency of magnitude 9 earthquakes, Geology, 36(3), 263.

McCaffrey, R. (2014), Interseismic locking on the Hikurangi subduction zone: Uncertainties from slow-slip events, Journal of Geophysical Research: Solid Earth, 119(10), 7874-7888.

McCaffrey, R., L. M. Wallace, and J. Beavan (2008), Slow slip and frictional transition at low temperature at the Hikurangi subduction zone, Nature Geoscience, 1(5), 316-320.

McGuire, J. J., and P. Segall (2003), Imaging of aseismic fault slip recorded by dense geodetic networks, Geophysical Journal International, 155, 778-788.

Meertens, C., F. Blume, H. Burgland, C. Puskas, J. Wahr, T. van Dam, and T. Herring (2012), Investigation of non-tectonic signals at GPS stations, in IGS Workshop, edited, Poland.

Melbourne, T. I. (2002), Precursory transient slip during the $2001 \mathrm{Mw}=8.4$ Peru earthquake sequence from continuous GPS, Geophysical Research Letters, 29(21).

Microsoft (2013), Excel, edited.

Misra, P., and P. Enge (2006), Global Positioning System - Signals, Measurement, and Performance, 569 pp., Ganga-Jumuna Press, Lincoln, Mass.

Mitsui, Y., and K. Hirahara (2008), Long-term slow slip events are not necessarily caused by high pore fluid pressure at the plate interface: an implication from two-dimensional model calculations, Geophysical Journal International, 174(1), 331-335.

Montgomery-Brown, E. K., P. Segall, and A. Miklius (2009), Kilauea slow slip events: Identification, source inversions, and relation to seismicity, Journal of Geophysical Research, 114.

Mountjoy, J. J., and P. M. Barnes (2011), Active upper plate thrust faulting in regions of low plate interface coupling, repeated slow slip events, and coastal uplift: Example from the Hikurangi Margin, New Zealand, Geochemistry, Geophysics, Geosystems, 12(1), 26.

Mousavian, R., and M. M. Hossainali (2014), Geodetic evidence on segmentation of Cascadia subduction zone based on episodic tremors and slips using multivariate harmonic analysis of GPS series, Stud. Geophys. Geod., 58, 376 - 387.

Murashev, A., and S. Palmer (1998), Geotechnical issues associated with development on Wellington's waterfront, IPENZ Transactions, 25(1/CE), 38 - 46.

Nicol, A., and L. M. Wallace (2007), Temporal stability of deformation rates: Comparison of geological and geodetic observations, Hikurangi subduction margin, New Zealand, Earth and Planetary Science Letters, 258(3-4), 397-413. 
Nicol, A., C. Mazengarb, F. Chanier, G. J. Rait, C. Uruski, and L. M. Wallace (2007), Tectonic evolution of the active Hikurangi subduction margin, New Zealand, since the Oligocene, Tectonics, 26(4).

Nocquet, J., P. A. Mothes, M. Vallee, and M. Regnier (2009), A slow slip event along the northern Ecuadorian subduction zone, in AGU 2009, edited.

Noda, H., and N. Lapusta (2013), Stable creeping fault segments can become destructive as a result of dynamic weakening, Nature, 493(7433), 518-521.

NZGS-GNS (2011), NZL GNS 1:1M Lithostratigraphy, GNS Science.

Obara, K. (2002), Nonvolcanic deep tremor associated with subduction in Southwest Japan, Science, 296(5573), 1679-1681.

Obara, K. (2010), Phenomenology of deep slow earthquake family in southwest Japan: Spatiotemporal characteristics and segmentation, Journal of Geophysical Research, 115.

Obara, K. (2011), Characteristics and interactions between non-volcanic tremor and related slow earthquakes in the Nankai subduction zone, southwest Japan, Journal of Geodynamics, 52(3-4), 229-248.

Ochi, T., and T. Kato (2013), Depth extent of the long-term slow slip event in the Tokai district, central Japan: A new insight, Journal of Geophysical Research: Solid Earth, 118(9), 4847-4860.

Ohnaka, M. (2013), The physics of rock failure and earthquakes, 270 pp., Cambridge University Press, Cambridge, UK.

Okada, Y. (1985), Surface deformation due to shear and tensile faults in a half-space, Bulletin of Seismological Society of America, 75(4), 1135-1154.

Okada, Y. (1992), Internal deformation due to shear and tensile faults in a half-space, Bulletin of Seismological Society of America, 82(2), 1018-1040.

Pacheco, J. F., L. R. Sykes, and C. H. Scholz (1993), Nature of seismic coupling along simple plate boundaries of the subduction type, Journal of Geophysical Research, 98(B8), 14133.

Paolucci, R. (1999), Numerical evaluation of the effect of cross-coupling of different components of ground motion in site response analyses, Bulletin of Seismological Society of America, 89(4), 877 - 887.

Papadopoulis, C. E., and H. Yeung (2001), Uncertainty estimation and Monte Carlo simulation method, Flow Measurement and Instrumentation, 12, 291 - 298.

Parthasarathy, P. R. (2007), Birth and death processes and order statistics, paper presented at Fifth SERC School on Special Functions and Functions of Matrix Argument: Recent Advances and Applications in Stochastic Processes, Statistics and Astrophysics, SERC, Centre for Mathematical Sciences, Trivandrum and Pala, India.

Peng, Z., and J. Gomberg (2010), An integrated perspective of the continuum between earthquakes and slow-slip phenomena, Nature Geoscience, 3(Sep 2010).

Perrin, N. D., and W. R. Stephenson (2010), Site class determinations (NZS 1170.5) in Wellington using borehole data and microtremor techniques, in 2010 NZSEE, edited, p. 8, Wellington. 
Pillans, B., and P. Huber (1992), Earthquake and uplift history of Miramar Peninsula, WellingtonRep., $11 \mathrm{pp}$, Victoria University of Wellington, Wellington.

Protti, M., V. Gonzalez, A. V. Newman, T. H. Dixon, S. Y. Schwartz, J. S. Marshall, L. Feng, J. I. Walter, R. Malservisi, and S. E. Owen (2013), Nicoya earthquake rupture anticipated by geodetic measurement of the locked plate interface, Nature Geoscience - Advance online publication(22 Dec 2013), 5.

Radiguet, M., F. Cotton, M. Vergnolle, M. Campillo, B. Valette, V. Kostoglodov, and N. Cotte (2011), Spatial and temporal evolution of a long term slow slip event: the 2006 Guerrero Slow Slip Event, Geophysical Journal International, 184(2), 816-828.

Radiguet, M., F. Cotton, M. Vergnolle, M. Campillo, A. Walpersdorf, N. Cotte, and V. Kostoglodov (2012), Slow slip events and strain accumulation in the Guerrero gap, Mexico, Journal of Geophysical Research, 117(B4).

Ramachandran, K., and R. D. Hyndman (2012), The fate of fluids released from subducting slab in northern Cascadia, Solid Earth, 3(1), 121-129.

Reyners, M., and P. McGinty (1999), Shallow subduction tectonics in the Raukumara Peninsula, New Zealand, as illuminated by earthquake focal mechanisms, Jnl. Geophys. Res., 104(B2), 3025 - 3034.

Reyners, M., and S. Bannister (2007), Earthquakes triggered by slow slip at the plate interface in the Hikurangi subduction zone, New Zealand, Geophysical Research Letters, 34(14).

Reyners, M., and D. Eberhart-Phillips (2009), Small earthquakes provide insight into plate coupling and fluid distribution in the Hikurangi subduction zone, New Zealand, Earth and Planetary Science Letters, 282(1-4), 299-305.

Reyners, M., D. Eberhart-Phillips, and S. Bannister (2011), Tracking repeated subduction of the Hikurangi Plateau beneath New Zealand, Earth and Planetary Science Letters, 311(1-2), 165171.

Reyners, M., D. Eberhart-Phillips, G. Stuart, and Y. Nishimura (2006), Imaging subduction from the trench to $300 \mathrm{~km}$ depth beneath the central North Island, New Zealand, with $\mathrm{Vp}$ and $\mathrm{Vp} / \mathrm{Vs}$, Geophysical Journal International, 165(2), 565-583.

Robinson, R. (1994), Shallow subduction tectonics and fault interaction: The Weber, New Zealand, earthquake sequence of 1990-1992, Journal of Geophysical Research, 99(B5), 9663.

Rodgers, D. W., and T. A. Little (2006), World's largest coseismic strike-slip offset: The 1855 rupture of the Wairarapa Fault, New Zealand, and implications for displacement/length scaling of continental earthquakes, Journal of Geophysical Research, 111(B12).

Rowan, C. J., A. P. Roberts, and G. J. Rait (2005), Relocation of the tectonic boundary between the Raukumara and Wairoa Domains (East Coast, North Island, New Zealand): Implications for the rotation history of the Hikurangi margin, New Zealand Journal of Geology and Geophysics, 48(1), 185-196.

Rowland, J. V., C. J. N. Wilson, and D. M. Gravley (2010), Spatial and temporal variations in magma-assisted rifting, Taupo Volcanic Zone, New Zealand, Journal of Volcanology and Geothermal Research, 190(1-2), 89-108.

Rubin, A. M. (2011), Designer friction laws for bimodal slow slip propagation speeds, Geochemistry, Geophysics, Geosystems, 12(4), n/a-n/a. 
Ruiz, S., M. Metois, A. Fuenzalida, J. Ruiz, F. Leyton, R. Grandin, C. Vigny, R. Madariaga, and J. Campos (2014), Intense foreshocks and a slow slip event preceded the 2014 lquique Mw 8.1 earthquake, Sciencexpress(24 Jul 2014), 7.

Rybicki, K. (1971), Elastic residual field of a very long strike-slip fault in the presence of a discontinuety, Bulletin of Seismological Society of America, 61(1), 79 - 92.

Sacks, S., S. Suyehiro, A. Linde, and J. A. Snoke (1978), Slow earthquakes and stress redistribution, Nature, 275.

Sacks, S., S. Suyehiro, A. Linde, and J. A. Snoke (1982), Stress redistribution and slow earthquakes, Tectonophysics, 81, 311-318.

Salmon, M. L., T. A. Stern, and M. K. Savage (2011), A major step in the continental Moho and its geodynamic consequences, Geophysical Journal International, 186, 32-44.

Sandwell, D. T., and W. H. F. Smith (2009), Global marine gravity from retracked Geosat and ERS-1 altimetry: Ridge segmentation versus spreading rate, Journal of Geophysical Research, 114(B1).

Santamaría-Gómez, A., M.-N. Bouin, X. Collilieux, and G. Wöppelmann (2011), Correlated errors in GPS position time series: Implications for velocity estimates, Journal of Geophysical Research, 116(B1).

Sato, K., N. Minagawa, M. Hyodo, T. Baba, T. Hori, and Y. Kaneda (2007), Effect of elastic inhomogeneity on the surface displacements in the northeastern Japan: Based on 3D numerical modelling, Earth Planets Space, 59, 1083 - 1093.

Savage, J. C. (1987), Effect of crustal layering upon dislocation modelling, Journal of Geophysical Research, 92(B10), 10595 - 10600.

Savage, J. C. (1998), Displacement field for an edge dislocation in a layered half-space, Journal of Geophysical Research, 103(B2), 2439 - 2446.

Savage, M. K., J. Park, and H. Todd (2007), Velocity and anisotropy structure at the Hikurangi subduction margin, New Zealand from receiver functions, Geophysical Journal International, 168(3), 1034-1050.

Schmidt, D. A., and H. Gao (2010), Source parameters and time-dependent slip distributions of slow slip events on the Cascadia subduction zone from 1998 to 2008, Journal of Geophysical Research, 115.

Scholz, C. H. (1998), Earthquakes and friction laws, Nature, 391(1 Jan 1998), 37 - 42.

Scholz, C. H. (2002), The mechanics of earthquakes and faulting, 2 ed., 471 pp., Cambridge University Press, Cambridge.

Scholz, C. H., and J. Campos (2012), The seismic coupling of subduction zones revisited, Journal of Geophysical Research, 117(B5).

Schwartz, S. Y., and J. M. Rokosky (2007), Slow slip events and seismic tremor at circumPacific subduction zones, Reviews of Geophysics, 45(3), 32.

Seebeck, H. C. (2012), Normal faulting, volcanism and fluid flow, Hikurangi subduction plate boundary, New Zealand, University of Canterbury, Christchurch. 
Seebeck, H. C., A. Nicol, M. Giba, J. Pettinga, and J. Walsh (2013), Geometry of the subducting Pacific plate since $20 \mathrm{Ma}$, Hikurangi margin, New Zealand, Journal of the Geological Society, 171(1), 131-143.

Segall, P. (2010), Earthquake and volcano deformation, 1 ed., 464 pp., Princeton University Press.

Semmens, S., N. D. Perrin, and P. Barker (2010a), What lies beneath: Geological and geotechnical characterisation of Wellington central commercial area, paper presented at IAEG, Taylor \& Francis.

Semmens, S., G. D. Dellow, and N. D. Perrin (2010b), It's our Fault - Geological and Geotechnical characterisation of the Wellington Central Business DistrictRep., 48 pp, GNS Science, Wellington.

Serpelloni, E., G. Casula, A. Galvani, M. Anzidei, and P. Baldi (2006), Data analysis of permanent GPS network in Italy and suurounding regions: Application of a distributed processing approach, Annals of Geophysics, 49(4-5).

Sgrillo, R. (2014), GE Graph, edited.

Skarbek, R. M., A. W. Rempel, and D. A. Schmidt (2012), Geologic heterogeneity can produce aseismic slip transients, Geophysical Research Letters, 39(21), L21306 - L21311.

Smith, E. F., and J. Gomberg (2009), A search in strainmeter data for slow slip associated with triggered and ambient tremor near Parkfield, California, Journal of Geophysical Research, 114.

Smith, E. G. C., T. Stern, and M. Reyners (1989), Subduction and back-arc activity at the Hikurangi convergent margin, New Zealand, Pageoph., 129(1/2).

Stagpole, V. (1997), Geophysical study of the Northern Taranaki basin, New zealand, Victoria University of Wellington, Wellington.

StatSoft, I. (2013), STATISTICA (data analysis software system), edited.

Steketee, J. A. (1958), On Volterra's dislocations in a semi-infiniteelastic medium, Can. Jnl. Phys., 36(2), 192 - 205.

Stern, T. A., and A. Benson (2011), Wide-angle seismic imaging beneath an andesitic arc: Central North Island, New Zealand, Journal of Geophysical Research, 116(B9).

Stern, T. A., W. Stratford, A. Seward, M. Henderson, M. K. Savage, E. G. C. Smith, A. Benson, S. Greve, and M. Salmon (2010), Crust-mantle structure of the central North Island, New Zealand, based on seismological observations, Journal of Volcanology and Geothermal Research, 190(1-2), 58-74.

Systat (2012), Tablecurve 2D, edited.

Thomas, M. Y., J.-P. Avouac, J. Champenois, J.-C. Lee, and L.-C. Kuo (2014), Spatiotemporal evolution of seismic and aseismic slip on the Longitudinal Valley Fault, Taiwan, Journal of Geophysical Research: Solid Earth, 119(6), 5114-5139.

Thornley, S. (1996), Neogene tectonics of Raukumara peninsula, northern Hikurangi Margin, New Zealand, 291 pp, Victoria University of Wellington, Wellington.

Tolvi, J. (1999), Outliers in time series - a review, doi: 10.1.1.198.823. 
Tsutsumi, A., O. Fabbri, A. M. Karpoff, K. Ujiie, and A. Tsujimoto (2011), Friction velocity dependence of clay-rich fault material along a megasplay fault in the Nankai subduction zone at intermediate to high velocities, Geophysical Research Letters, 38(19), L19301 - L19307.

Udias, A., R. Madariaga, and E. Buforn (2014), Source mechanisms of earthquakes: theory and practice, 302 pp., Cambridge University Press, Cambridge.

Ujiie, K., et al. (2013), Low coseismic shear stress on the Tohoku-Oki megathrust determined from laboratory experiments, Science, 342(6163), 1211-1214.

Unistat (2014), UNISTAT Statistical Package, edited.

Upton, P., P. O. Koons, and D. Eberhart-Phillips (2003), Extension and partitioning in an oblique subduction zone, New Zealand: Constraints from three-dimensional numerical modeling, Tectonics, 22(6).

Vallée, M., et al. (2013), Intense interface seismicity triggered by a shallow slow slip event in the Central Ecuador subduction zone, Journal of Geophysical Research: Solid Earth, 118(6), 29652981.

Vidale, J. E., A. J. Hotovec, A. Ghosh, K. C. Creager, and J. Gomberg (2011), Tiny intraplate earthquakes triggered by nearby episodic tremor and slip in Cascadia, Geochemistry, Geophysics, Geosystems, 12(6), n/a-n/a.

Villegas Lanza, J., J.-M. Nocquet, M. Chlieh, M. Vallee, H. Tavera, and F. Rolandone (2012), A Slow Slip Event and synchronous seismicity in the northern Peru subduction zone, in AGU, edited, San Francisco.

Vollmer, F. W. (2012), Orient, edited.

Volterra, V. (1907), Ann. Sci. de l'Ec. Norm. Sup (3), 24, 401 - 517.

Walcott, R. I. (1984), The kinematics of the plate boundary zone through New Zealand: a comparison of short- and long-term deformations, Geophys. J. R astr. Soc., 79, 613 - 633.

Walcott, R. I. (1993), Extensional mechanics of continental lithosphere, Annali di Geofisica, $36(2)$.

Wald, D. J., and R. W. Graves (2001), Resolution analysis of finite fault source inversion using one- and three-dimensional Green's functions: 2. Combining seismic and geodetic data, Journal of Geophysical Research, 106(B5), 8767.

Wallace, L. M., and J. Beavan (2006), A large slow slip event on the central Hikurangi subduction interface beneath the Manawatu region, North Island, New Zealand, Geophysical Research Letters, 33(11).

Wallace, L. M., and J. Beavan (2010), Diverse slow slip behavior at the Hikurangi subduction margin, New Zealand, Journal of Geophysical Research, 115(B12).

Wallace, L. M., and D. Eberhart-Phillips (2013), Newly observed, deep slow slip events at the central Hikurangi margin, New Zealand: Implications for downdip variability of slow slip and tremor, and relationship to seismic structure, Geophysical Research Letters, 40(20), 5393-5398.

Wallace, L. M., J. Beavan, R. McCaffrey, and D. J. Darby (2004), Subduction zone coupling and tectonic block rotations in the North Island, New Zealand, Journal of Geophysical Research, 109(B12). 
Wallace, L. M., J. Beavan, S. Bannister, and C. A. Williams (2012a), Simultaneous long-term and short-term slow slip events at the Hikurangi subduction margin, New Zealand: Implications for processes that control slow slip event occurrence, duration, and migration, Journal of Geophysical Research, 117(B11).

Wallace, L. M., U. Cochran, W. L. Power, and K. Clark (2014), Earthquake and Tsunami Potential of the Hikurangi Subduction Thrust, New Zealand: Insights from Paleoseismology, GPS, and Tsunami Modeling, Oceanography, 27(2), 104-117.

Wallace, L. M., P. Barnes, J. Beavan, R. Van Dissen, N. Litchfield, J. Mountjoy, R. Langridge, G. Lamarche, and N. Pondard (2012b), The kinematics of a transition from subduction to strikeslip: An example from the central New Zealand plate boundary, Journal of Geophysical Research, 117(B2).

Wallace, L. M., et al. (2009), Characterizing the seismogenic zone of a major plate boundary subduction thrust: Hikurangi Margin, New Zealand, Geochemistry, Geophysics, Geosystems, 10(10), 32.

Wang, K., and S. L. Bilek (2014), Invited review paper: Fault creep caused by subduction of rough seafloor relief, Tectonophysics, 610, 1-24.

Wang, R., F. L. Martın, and F. Roth (2003), Computation of deformation induced by earthquakes in a multi-layered crust - FORTRAN programs EDGRN/EDCMP, Computers \& Geosciences, 29, 195 - 207.

Webb, T. H., and H. Anderson (1998), Focal mechanisms of large earthquakes in the North Island of New Zealand: slip partitioning at an oblique active margin, Geophys. J. Int., 134, 40 86.

Wech, A. G. (2010), Tremor, 117 pp, University of Washington.

Wech, A. G., and N. M. Bartlow (2014), Slip rate and tremor genesis in Cascadia, Geophysical Research Letters, 41(2), 392-398.

Wech, A. G., K. C. Creager, H. Houston, and J. E. Vidale (2010), An earthquake-like magnitude-frequency distribution of slow slip in northern Cascadia, Geophysical Research Letters, 37(22), n/a-n/a.

Wech, A. G., C. M. Boese, T. A. Stern, and J. Townend (2012), Tectonic tremor and deep slow slip on the Alpine Fault, Geophysical Research Letters, 39(10).

Wei, M., J. J. McGuire, and E. Richardson (2012), A slow slip event in the south central Alaska Subduction Zone and related seismicity anomaly, Geophysical Research Letters, 39(15).

Wei, M., Y. Kaneko, Y. Liu, and J. J. McGuire (2013), Episodic fault creep events in California controlled by shallow frictional heterogeneity, Nature Geoscience, 6(7), 566-570.

Williams, C. A., D. Eberhart-Phillips, S. Bannister, D. H. N. Barker, S. Henrys, M. Reyners, and R. Sutherland (2013), Revised Interface Geometry for the Hikurangi Subduction Zone, New Zealand, Seismological Research Letters, 84(6), 1066-1073.

Williams, S. D. P. (2003), The effect of coloured noise on the uncertainties of rates estimated from geodetic time series, Journal of Geodesy, 76(9-10), 483-494.

Wood, J. H., and G. R. Martin (1995), Evaluation of seismic design parameters for the Museum of New Zealand site, Bull. N. Z. Nat. Soc. Earthquake Eng., 28(2), 118 - 133. 
Wood, R., and B. Davy (1994), The Hikurangi Plateau, Marine Geology, 118, 153 - 173.

Wright, C. (2008), Station corrections for the Kaapvaal seismic network: Statistical properties and relation to lithospheric structure, Physics of the Earth and Planetary Interiors, 167(1-2), 3952.

Xu, S. (2014), A Property of Geometric Mean Regression, The American Statistician, 68(4), 277-281.

Yabuki, T., and M. Matsu'ura (1992), Geodetic data inversion using a Bayesian information criterion for spatial distribution of fault slip, Geophysical Journal International, 109, 363 - 375.

Yin, X., J. Goudriaan, E. A. Lantinga, J. Vos, and H. J. Spiertz (2003), A Flexible Sigmoid Function of Determinate Growth, Annals of Botany, 91(3), 361-371.

Yokota, Y., and K. Koketsu (2015), A very long-term transient event preceding the 2011 Tohoku earthquake, Nature Communications, 6(5934), 5.

Yoshioka, S., T. Mikumo, V. Kostoglodov, K. M. Larson, A. R. Lowry, and S. K. Singh (2004), Interplate coupling and a recent aseismic slow slip event in the Guerrero seismic gap of the Mexican subduction zone, as deduced from GPS data inversion using a Bayesian information criterion, Physics of the Earth and Planetary Interiors, 146(3-4), 513-530.

Zeng, Y., and R. Benites (1998), Seismic response of multi-layered basins with velocity gradients upon incidence of plane shear waves, Earthquake Engineering and Structural Dynamics, 27, 15 - 28.

Zhu, L., and L. A. Rivera (2002), A note on the dynamic and static displacements from a point source in a layered media, Geophys. J. Int., 148, 619 - 627. 


\section{Appendix 1 - Geological legend for Fig. 2.3}

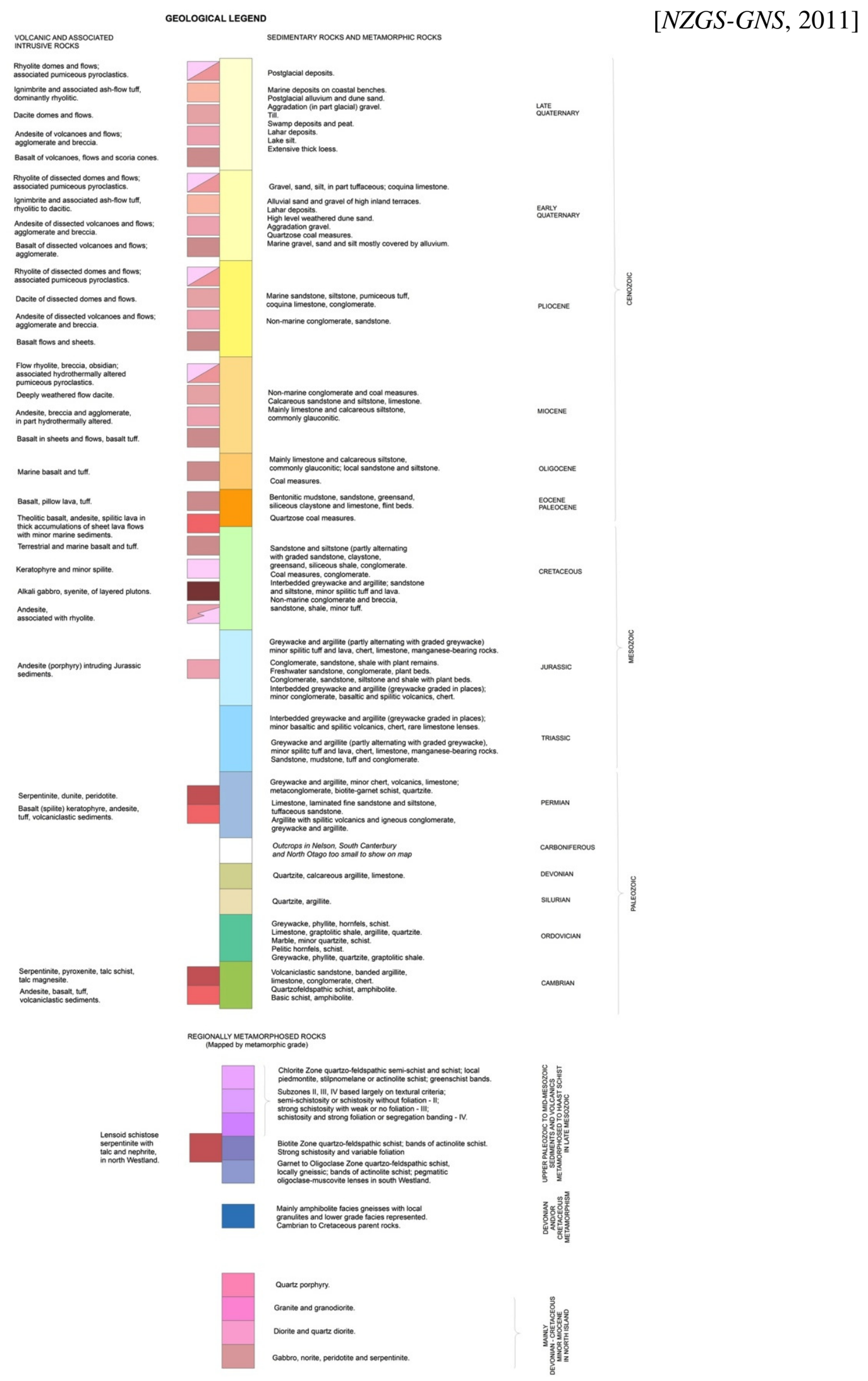




\section{Appendix 2 - Noise processing schema (ref. 2.4.2)}

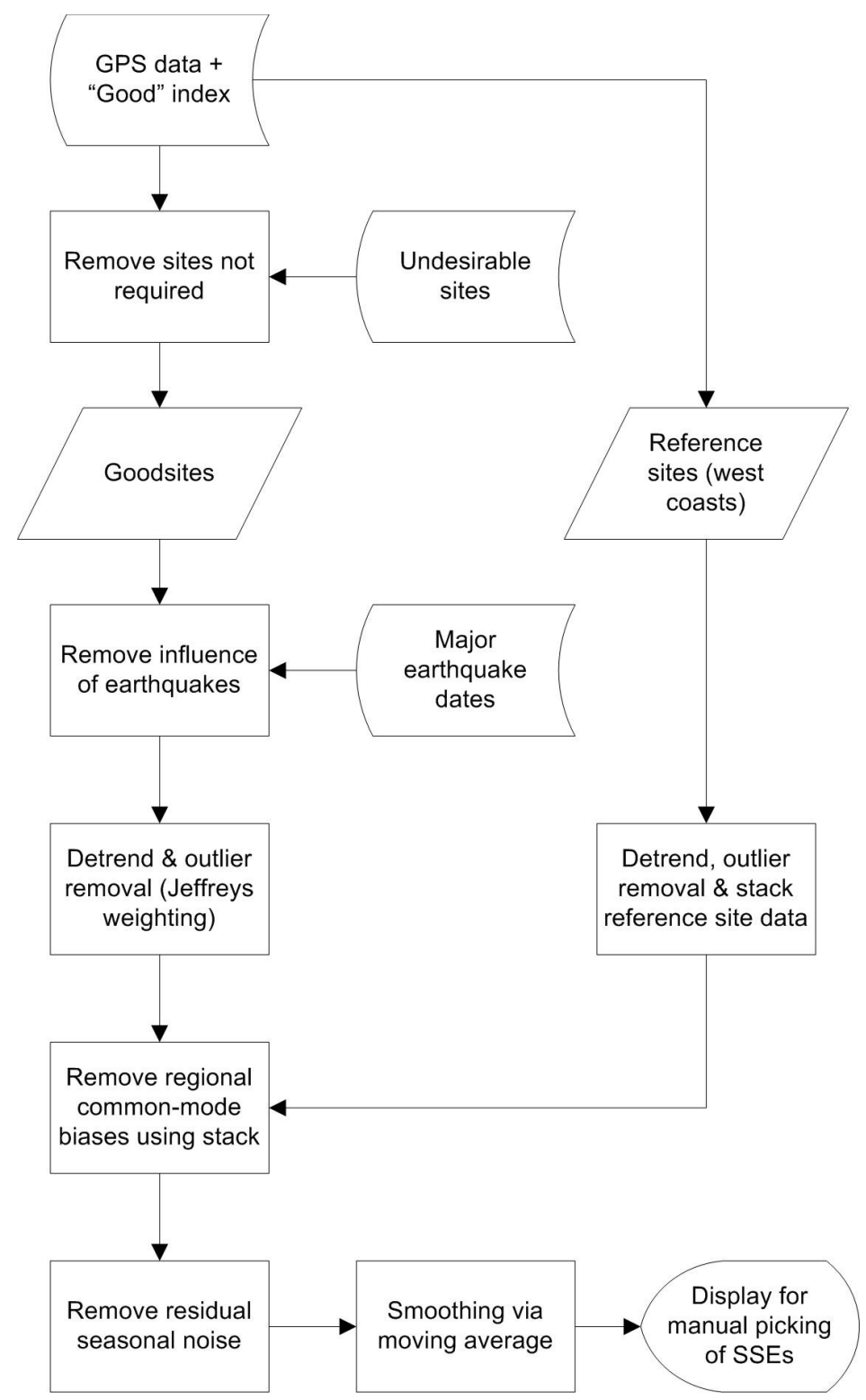




\section{Appendix 3 - Spectral components of the regional stack}
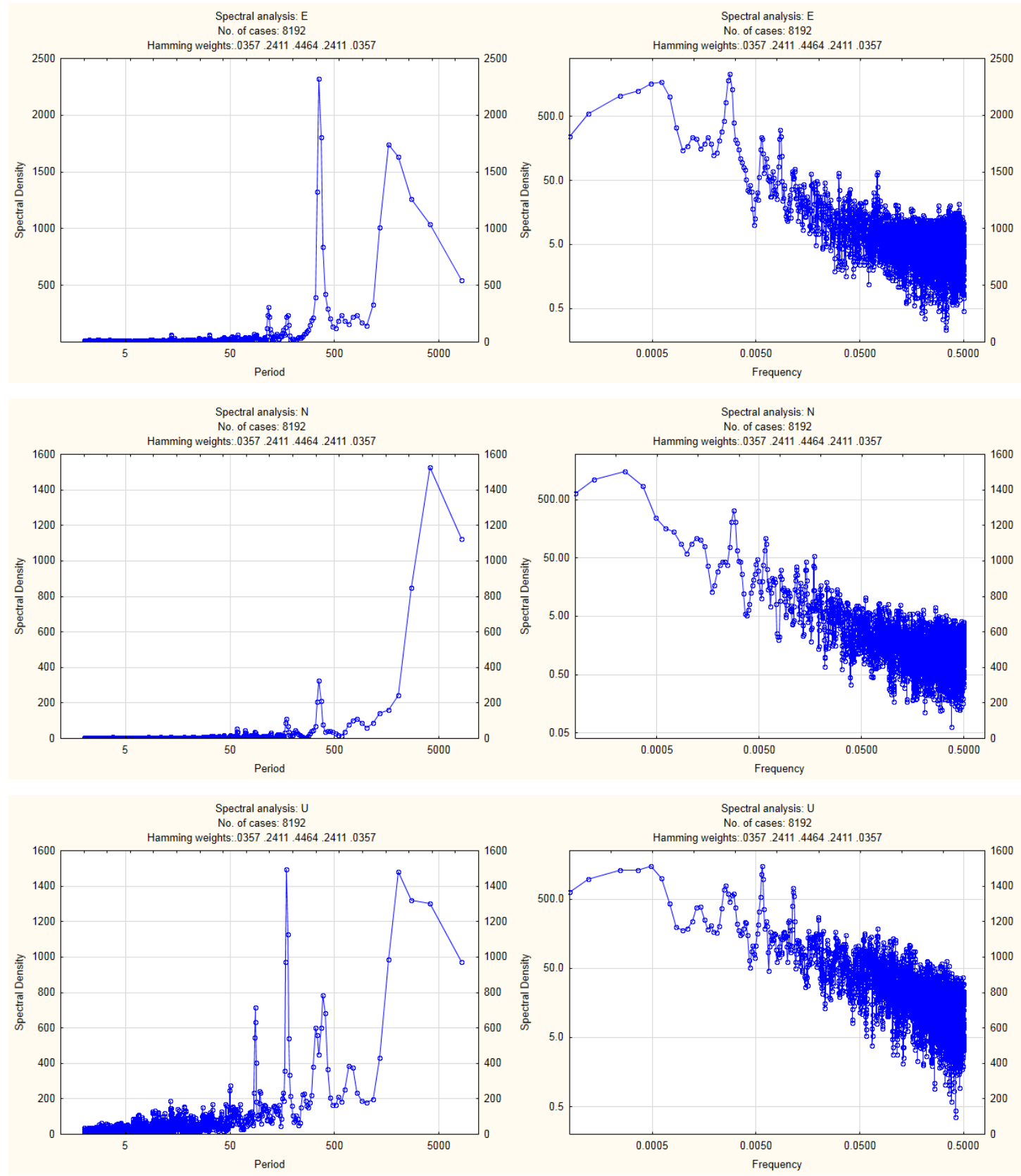

Plots in the left column show the spectral density for the 3 components against period in days. Plots on the right are of spectral density against frequency in day ${ }^{-1}$. An approximately annual signal is pronounced in the $\mathrm{E}$ and $\mathrm{U}$ components, and an approximately 6-monthly signal in the U component. (ref. 3.1.1). The long-period peak requires further investigation in view of recent investigations following the 2011 Tohoku-oki earthquake [Yokota and Koketsu, 2015]. 


\section{Appendix 4 - Jeffreys' Weighting and outlier treatment}

(ref. 2.4.2.4) Example of the effect of application of optimised Jeffreys' weighting on the E component cumulative residual distributions from a typical site - AUCK. Dashed lines show the cumulative normal distribution.
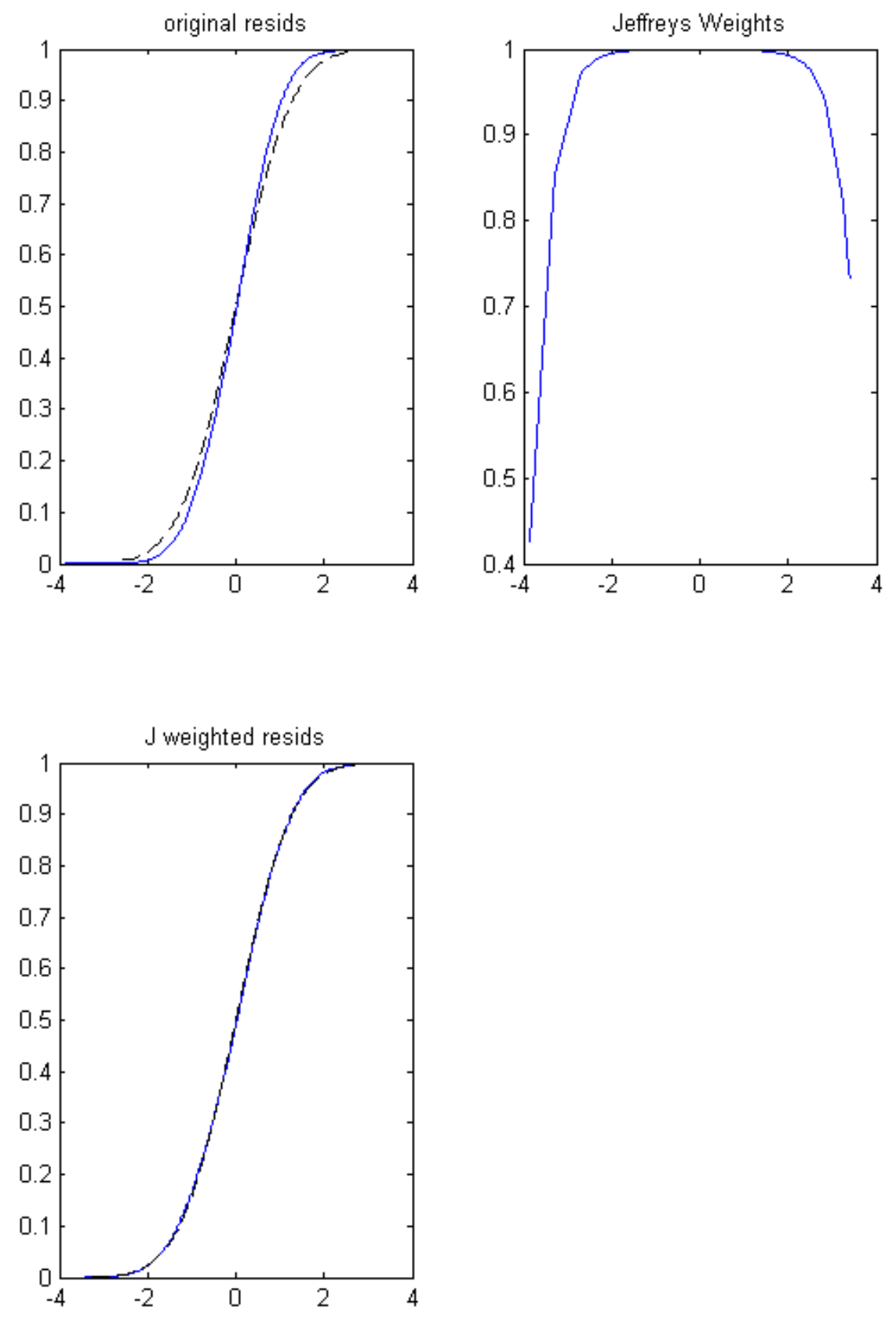
The lower plot shows that the residuals have been normalized after application of the asymmetric weighting shown in the upper right. The plots below show rms values of residuals after Jeffreys' weighting, plotted against rms values before weighting, for each component, all sites:
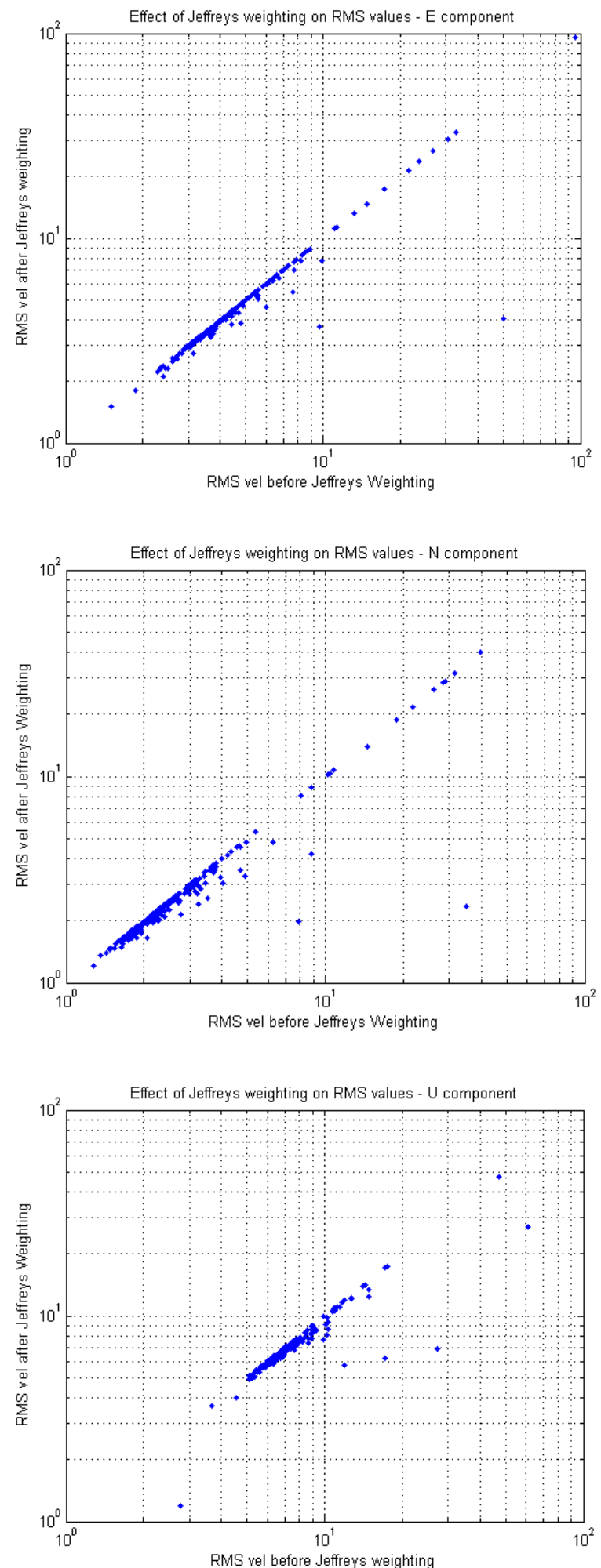


\section{Appendix 5 - Inversion methods used for SSEs in other}

\section{subduction zones (ref. 2.6.2)}

Outside New Zealand, a variety of techniques have been used to perform inversion:

Japan

A great deal of the Japanese work on SSEs appears to use associations between SSEs and other seismic activity, such that their high-resolution high-density seismometer and borehole strainmeter network data serve as the main basis for analyses. GPS data analysis methods do not seem to have been widely documented. Ochi and Kato [2013] looked at long-duration events (>23 months) and emphasized the errors that could occur if the wrong "baseline" steady-state deformations were used in analysis, therefore preferring to use un-detrended data. They proposed an 8-component (tectonic + nontectonic) displacement equation, and referred back to inversion methods in Yabuki and Matsu'ura [1992], which describes a relatively complex Bayesian approach, but essentially relying on Green's functions from Okada [1985].

Taiwan

Whilst triggered tremor and SSE have been detected in Taiwan [Chao, 2012; C Liu et $a l .$, 2009], I have found no full reports of inversions of SSE data. One abstract refers only to a 'uniform stress drop inversion' [Ching et al., 2014]. Co-seismic deformations have, however, been inverted for a very dense GPS network using a technique called 'empirical orthogonal function' or EOF, which is a form of principal components analysis [Chang and Chao, 2014]. A Principal Component Analysis-based Inversion Method (PCAIM) has also been used by Thomas et al. [2014] to characterize the spatiotemporal behavior of the combined seismic and aseismic deformation. Other authors used Wang's EDGRN/EDCMP code [R Wang et al., 2003] for co-seismic analysis, but noted that this does not allow for parameter variation along the source [Huang et al., 2013]. 


\section{Alaska}

Wei et al. [2012] used a Network Strain Filter to detect strain transients, using a formulation with 6 component functions encompassing both tectonic and non-tectonic processes. Inversion used Green's functions based on a dislocation model, and formulae based on Okada [1985]. Fu and Freymueller [2013] noted that both log and exponential functions fitted relaxation times equally well. Their subduction zone interface model was based on Hayes et al. [2012]. Greens functions were taken from Okada [1985]. They refer back to inversion methods of Hreinsdóttir et al. [2006] that were used on co-seismic magnitudes of displacements of metres rather than millimetres, with minimization of a misfit - roughness difference function.

\section{Cascadia}

McGuire and Segall [2003] described their procedure in detail. They used a network inversion filter (NIF) procedure with an extended Kalman filter, which used 4 terms plus error to describe the surface displacements as a function of time. The filter is based on the premise that tectonic deformation is spatially correlated between adjacent stations, whereas benchmark motions are uncorrelated. This information is used to filter out the uncorrelated trends and random noise, and estimate the fault slip that best models the temporally and spatially correlated surface deformation. Their interface model was a $3 \mathrm{D}$ surface discretized at $5 \mathrm{~km}$ intervals with 72 rectangular fault segments of approx. $25 \times 15 \mathrm{~km}$ area. Inversion was based on an elastic half space and used Green's functions tabulated by Okada [1985]. Schmidt and Gao [2010] used a similar NIF procedure in order to carry out systematic inversion of multiple events, and calculated Green's functions used boundary element code ('Poly3D') with $\sim 25 \mathrm{~km}$ triangular elements.

\section{Costa Rica}

Feng et al. [2012], Jiang et al. [2012], and Protti et al. [2013] used a 2D model of the subduction interface, discretized to 30 x $40 \mathrm{~km}$ patches on which the Okada [1992] dislocation model was applied. Feng's paper gives details of pretreatments used to reduce noise. 


\section{Mexico}

Early work used guided parameter-space inversions for both the moving and static Okada dislocations [Lowry et al., 2001b]. Subsequently, work on a large event in the Guerrero Gap also used an inversion technique based on Bayesian information criteria [Yoshioka et al., 2004]. More recent work Cavalié et al. [2013] on both InSAR and GPS data utilizes the Radiguet et al. [2011] methodology. Unusually, this uses a layered crustal model and Green's functions for a layered half-space.

\section{Ecuador, Peru and Chile}

Single site inversions are noted in reports from Melbourne [2002], and Vallée et al. [2013]. For the Central Ecuador SSE, Vallée et al. [2013] used Radiguet's method of inversion. Okada's formulae were used in the inversion of the SSE preceding the $\mathrm{M}_{\mathrm{w}}$ 8.1 April 2014 Iqique earthquake [Ruiz et al., 2014].

\section{Non-specific:}

Colella et al. [2012] were primarily concerned with modeling a rate and state simulation of slip, and used dislocation solutions based on Green's functions for rectangular fault elements from Okada [1992].

Crowell et al. [2012] were concerned with real-time inversion of $1 \mathrm{~Hz}$ GPS data to provide warning systems, using either pre-defined fault systems or trial faults generated by moment tensor inversion. In both cases Green's functions were obtained from Okada [1985]. The published work is primarily based on a subsequently-published $\mathrm{PhD}$ thesis [Crowell, 2013]. 


\section{Appendix 6 - Comparison of interface models (ref. 2.6.1)}

The interface model of $C$ A Williams et al. [2013] is compared with one produced using Matlab code written by Prof. E Smith and utilized in Lamb and Smith [2013]. The Williams et al. model has been reduced to NZTM coordinates in order to carry out the comparison, using the following procedure:

1. Convert from the downloaded GMT .grd format to a text array using Matlab grdread2.m

2. Import the text array to a spreadsheet

3. Downsample/smooth from $0.01 \mathrm{deg}$ resolution by averaging over $0.1 \mathrm{deg}$ rectangles

4. Use the Excel function HLOOKUP to extract coordinates corresponding to $10 \mathrm{~km}$ depth increments (note an imprecision of $+/-5 \mathrm{~km}$ in this process)

5. Tabulate latitude and longitude in separate arrays

6. Use an extract from Matlab SSEinvert.m to convert the latitude and longitude arrays to NZTM, and then rotate to the same strike as Smith's array

7. Extract latitude and longitude in the new rotated coordinates

8. Round the latitudes to $50 \mathrm{~km}$ increments

9. Average the longitude values in each $50 \mathrm{~km}$ bin

10. Assemble in the same format as Smith's Benioff5_85.xlsx -> GNSsubduct.xlsx

In both cases, blue crosses and lines show $10 \mathrm{~km}$ depth contours, and the tan crosses and lines represent cylindrical extrapolation from the $30 \mathrm{~km}$ depth contour to the trench position shown by the magenta lines (as used in Smith's code).

\section{Smith:}

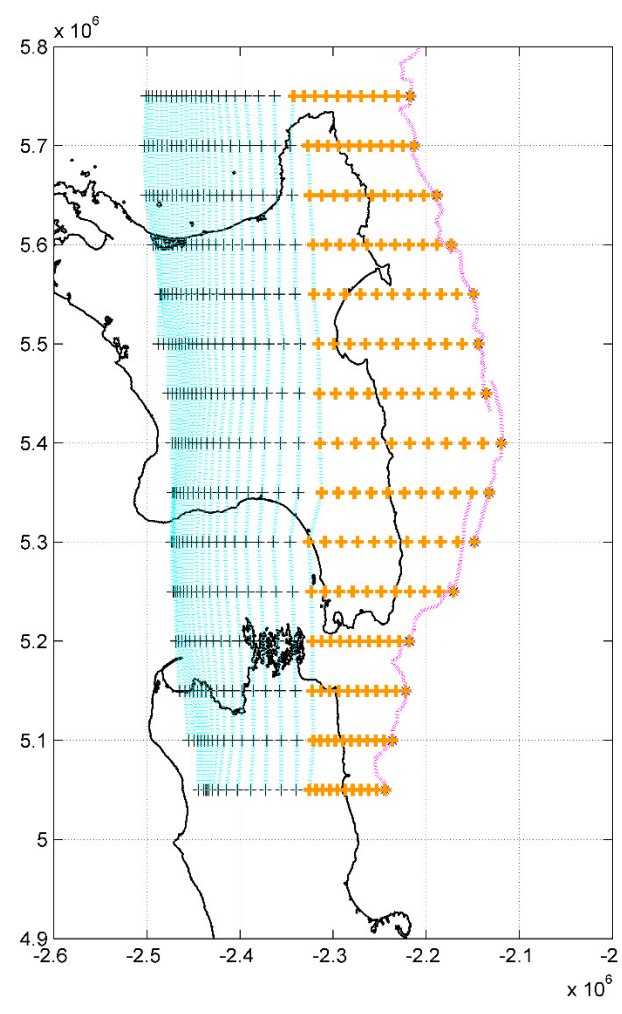

\section{Williams:}

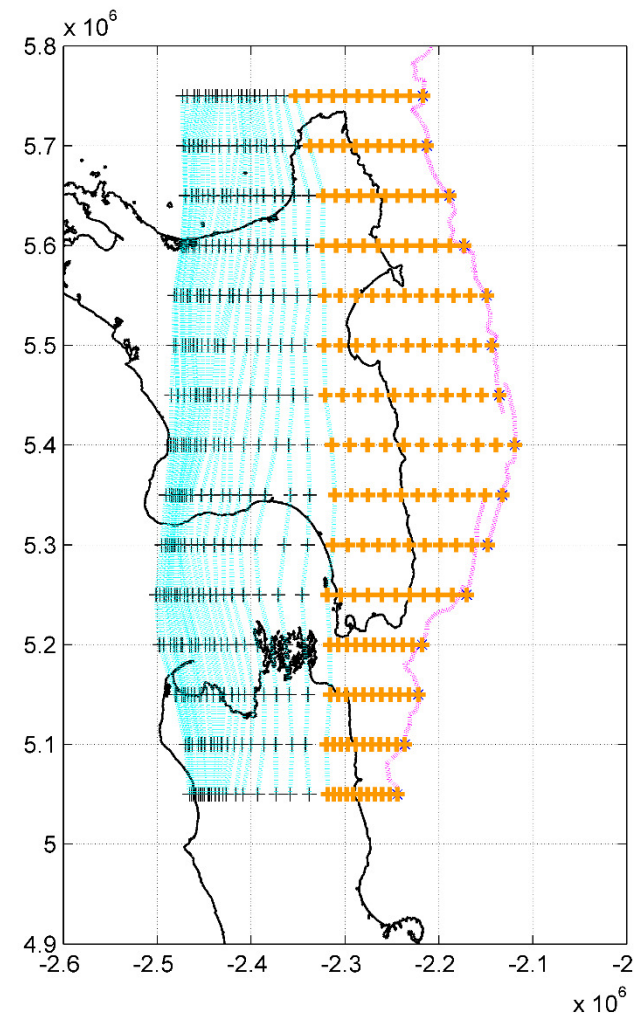


Whilst the contour plots show a good deal of similarity, there are differences that are more easily seen when the eastings differences at each contour depth grid point are plotted (Williams et al. minus Smith):

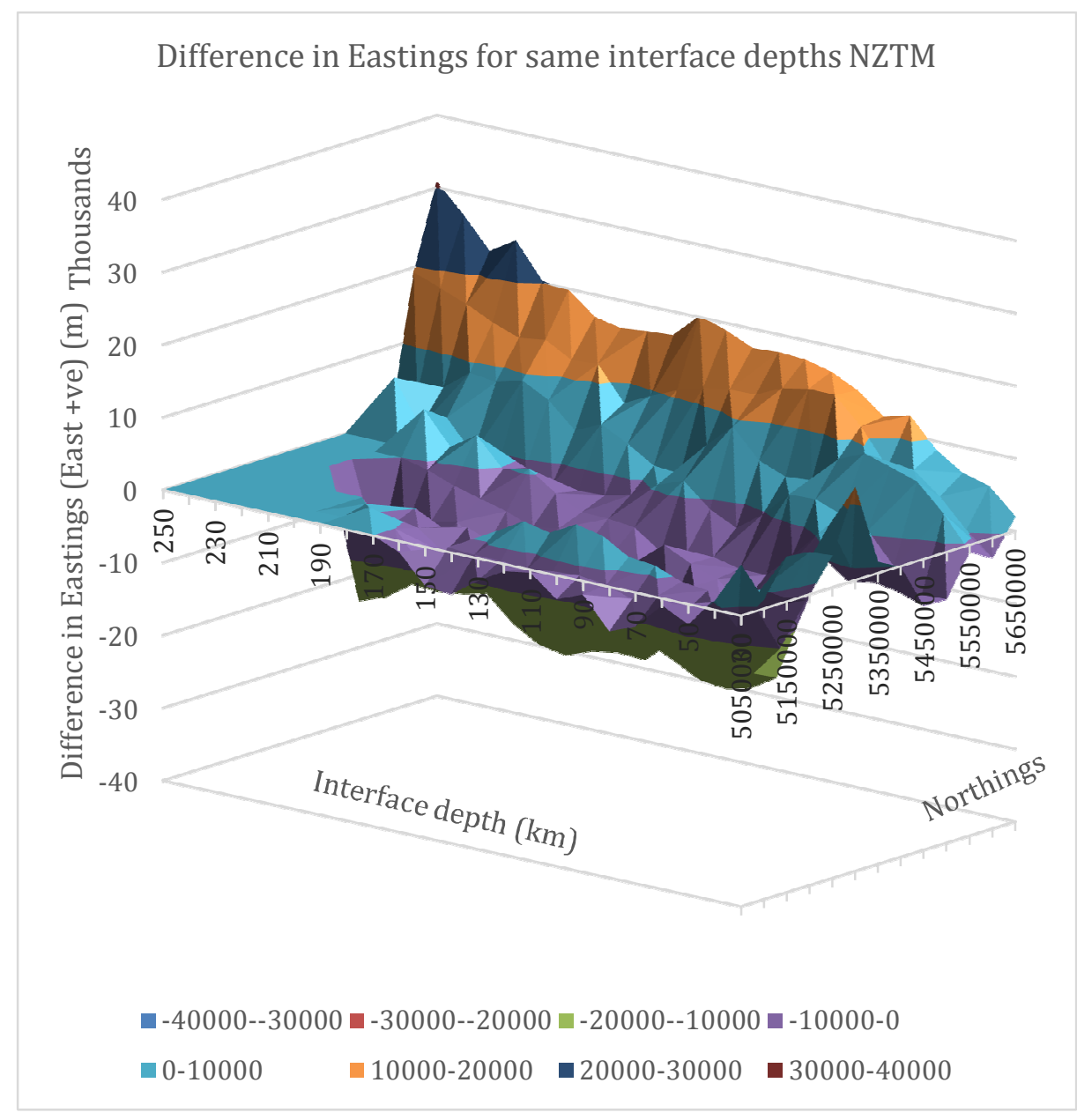

It can be seen that whilst for most of the region, the differences in eastings for the same interface depth are within $+/-10 \mathrm{~km}$, in the region of the Wanganui basin, the same depth contours are located between 10 and $20 \mathrm{~km}$ more westerly in the Williams model. In the north, the deeper contours are located up to $30 \mathrm{~km}$ more towards the east in the Williams model.

Both models have been used in preliminary inversion tests. In practical terms there was no significant difference between the two outcomes. 


\section{Appendix 7 - Validation of Matlab implementation of Okada code, and EDGRN/EDCMP code (ref. 2.6.2)}

\section{A Homogenous half-space codes}

The Matlab code used in this work was written by Prof. E. Smith based on Okada's code for surface displacements for a buried fault in an homogenous half-space. This has been validated by using specific examples and comparing with the output from a compiled version of Okada's original Fortran code.

Okada published a complete set of closed analytical expressions in a unified manner for the internal displacements and strains due to shear and tensile faults in a half-space for both point and finite rectangular sources [Okada, 1992]. He also published Fortran coding for his routines $\underline{\underline{6}}$, which have subsequently been accepted almost universally for inversion projects. The DC3D routine has been implemented in Matlab by E Smith as okada1.m.

Smith's implementation has been validated by the following processes:

1. Using the single point case given by Okada as an example for testing, and

2. Reproducing a specific example from the literature

3. Comparing Smith's pseudo-3D meshed displacement fields with fields generated by Wang's implementation of Okada

In order to utilize the Fortran code in an interactive mode, a small header was added, courtesy R. Benites (Appendix 7.1), and the code compiled for Windows 8.1, using GNU gfortran ${ }^{\underline{7}}$, as okada1.exe (which is then run under DOS)

1. For the first validation, the test case given in Okada's manual for the software (Appendix 7.2) was input both to okada1.exe and to the Matlab code okadala.m. It is noted that the Matlab implementation assumes that the buried fault has bottom left located at $0,0, \mathrm{~d}$, where $\mathrm{d}$ represents depth and is in the $-\mathrm{z}$ direction. The code

\footnotetext{
$\underline{6}$ http://www.bosai.go.jp/study/application/dc3d/DC3Dhtml_E.html

${ }^{7}$ http://gcc.gnu.org/wiki/GFortranBinaries
} 
was set up to output displacement and strain along the $\mathrm{y}$ axis at the $\mathrm{x}$-axis midplane of the fault.

Test case results:

\begin{tabular}{|l|l|l|l|}
\hline & Ux & Uy & Uz \\
\hline Reference & -37.898 & 63.179 & 14.961 \\
\hline Okada1.exe & -37.898 & 63.178 & 14.958 \\
\hline Matlab (read from plot) & -37.935 & 63.216 & 14.957 \\
\hline
\end{tabular}

2. For the $2^{\text {nd }}$ validation, the example shown as figure 3 in Cattin et al. [1999] was used, which comprises a normal fault striking along $+\mathrm{y}$, buried at a depth of $3 \mathrm{~km}$ to the upper bound. The coordinate system had to be re-arranged since these authors used the opposite-handed convention to Okada. Figure 3 in the paper was digitized. The appropriate parameters were also input to okada1.m and okada1.exe. The comparisons are shown in the following figures. It can be seen that the point values from okada1.exe satisfactorily validated the Matlab code, although both gave values that departed slightly from the Cattin curves. Whilst it was initially assumed that a default value of 0.6667 could be used for alpha, it was subsequently noted that whilst the authors did not quote alpha explicitly, they gave enough information for it to be able to calculated (Young's modulus E $=10^{11} \mathrm{~Pa}$, Poisson's ratio $v=0.27$ and density $\rho=2800 \mathrm{~kg} / \mathrm{m}^{3}$, giving a value for alpha of 0.685 - this difference had only a very minor effect on the results and was not sufficient to explain the discrepancy. The authors also used a fault length of $28.2 \mathrm{~km}$ as compared with $30 \mathrm{~km}$ taken from their "L/W 3", and this may also be a contributory factor. However, when these slightly amended values were used in the Matlab code, the differences were negligible (see points labelled Matlab2).

Over 13 ' $y$ ' values for which there were exact correspondences, the average difference between the Matlab and the Fortran code result was -0.006 (sd 0.054) and -0.010 (sd 0.097) for the horizontal and vertical values respectively. Neither of these averages is significantly different to zero at the 0.05 level.

Also shown in the following figures is the comparison undertaken to validate the homogenous half-space option (based on Okada) in the EDCMP code of $R$ Wang et al. [2003]. 

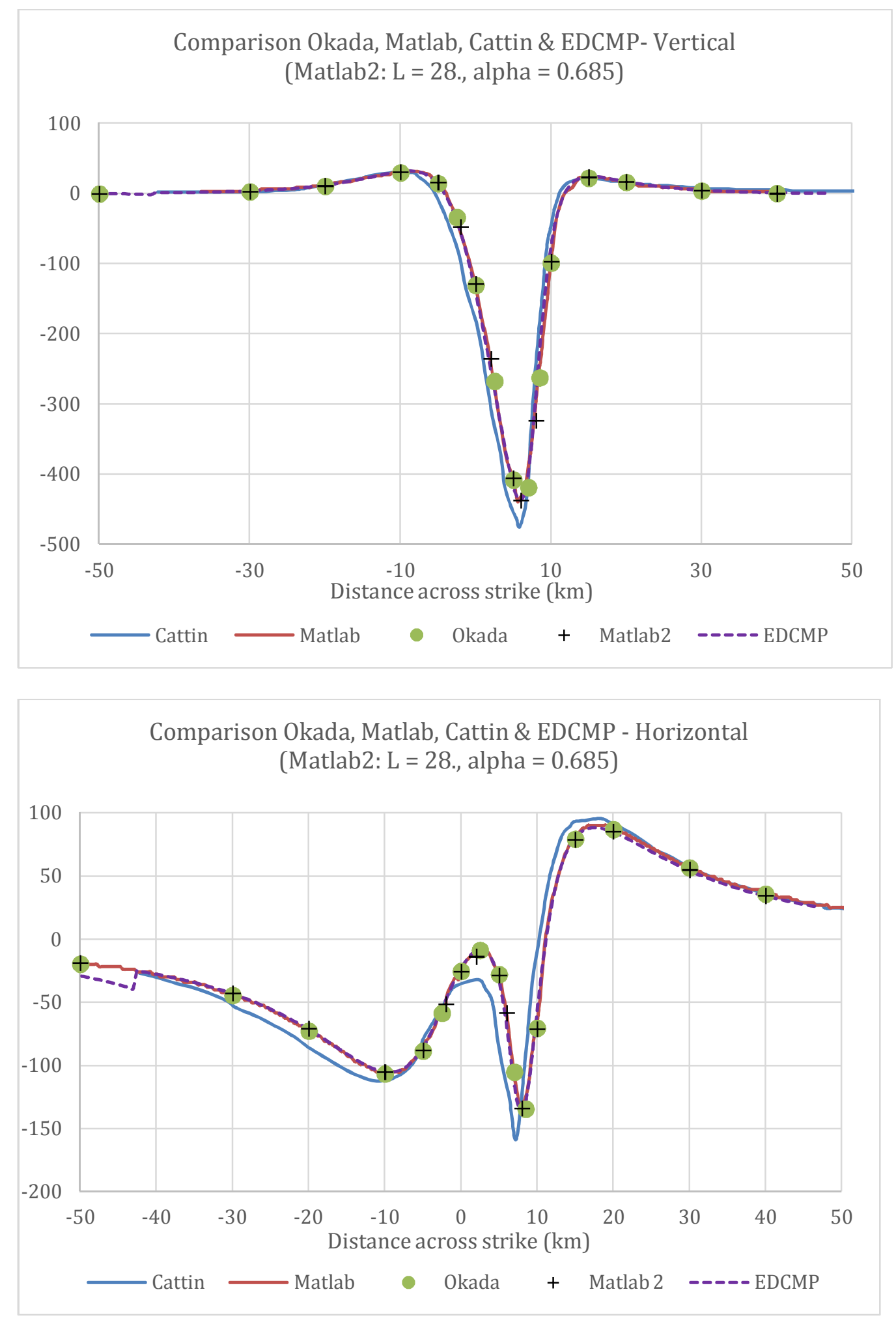

Whilst the discrepancy with the digitized Cattin figures remains unexplained, both the Matlab code and the homogenous half-space code in EDCMP were considered validated against the original Okada fortran calculations. 
3. During coding of the inversion process, Smith's Matlab implementation of Okada's DC3D was also compared with the output from Wang's implementation of the same Okada code. The following example comparison shows the $\mathrm{N}$ component of surface displacements from a $5 \times 5.7 \mathrm{~km}$ fault at $45 \mathrm{~km}$ depth and $29^{\circ} \mathrm{dip}$, slipping $1 \mathrm{~m}$ updip. Left to right, the images show Smith's output over an area of $200 \times 200 \mathrm{~km}$ in plan; then displacements across strike; compared with output from Wang's EDGRN/EDCMP, shown in plan over the central $100 \mathrm{x} 100 \mathrm{~km}$ area. The comparisons were carried out over all 3 components and for both dip slip and strike slip movements, and the agreement was considered satisfactory. Simulated vectors at 5 specific cGPS site locations were also validated against the mesh plots.
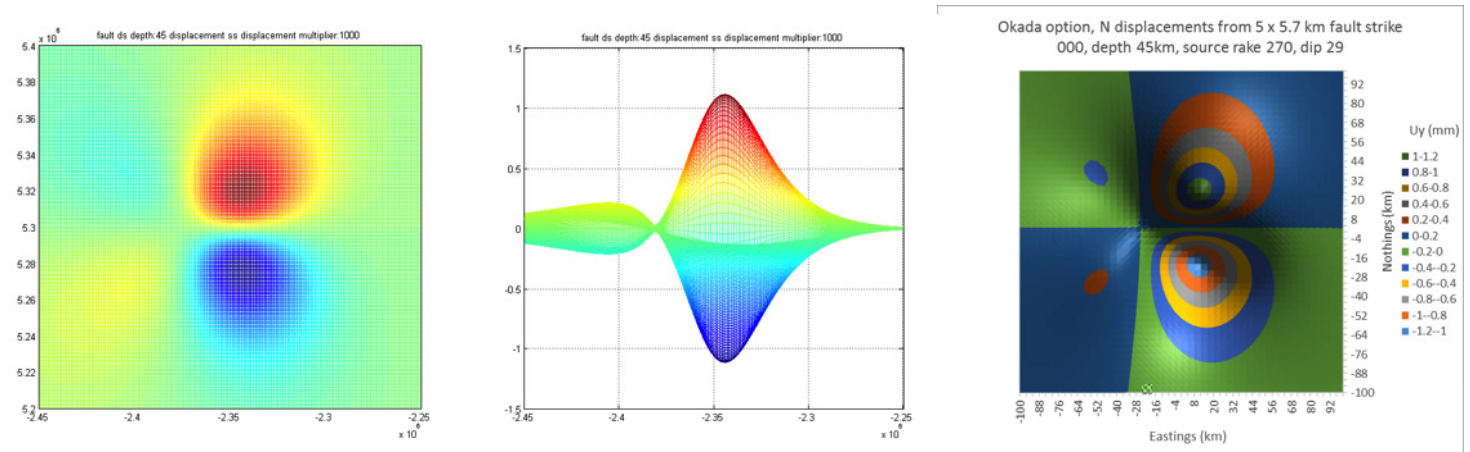

\section{B EDGRN/EDCMP layered code}

In order to validate the layer code, the same data were used from Fig. 1 and Fig. 5 of Cattin et al. [1999], with a ratio of $\mathrm{E}^{\mathrm{E}} \mathrm{E}^{\prime}=0.1$. The figures below have been formatted in a similar manner as Fig. 5 of Cattin et al.

In these plots, the dashed lines indicate the digitized data from the Cattin et al. plots, and the solid blue lines show the corresponding results from EDGRN/EDCMP.

It can be seen that whilst the peaks are not as sharp as those in Cattin et al., which is probably a consequence of different array resolutions, in other respects the magnitudes of the effect and the essential features are very similar. 

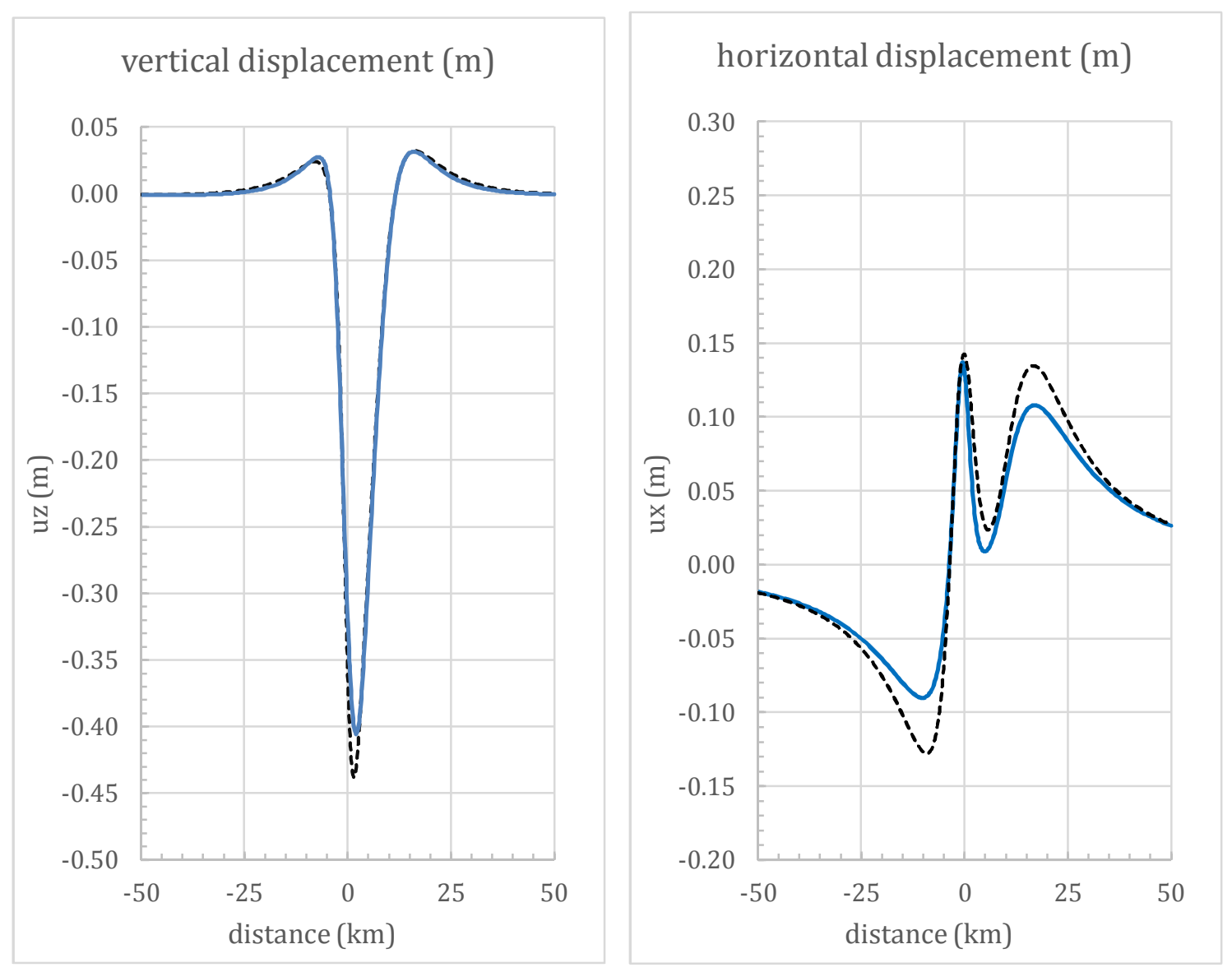

Finally, to ensure that the two parts of the EDGRN/ECMP code were consistent, a layered model, which was considered to simulate the shallowest parts of the NE section of the Hikurangi subduction geometry, was run with trivial differences in the elastic parameters, in order to simulate an homogenous half-space model, and the differences compared against the Okada results (plots as per explanation Fig. 4.4a): 


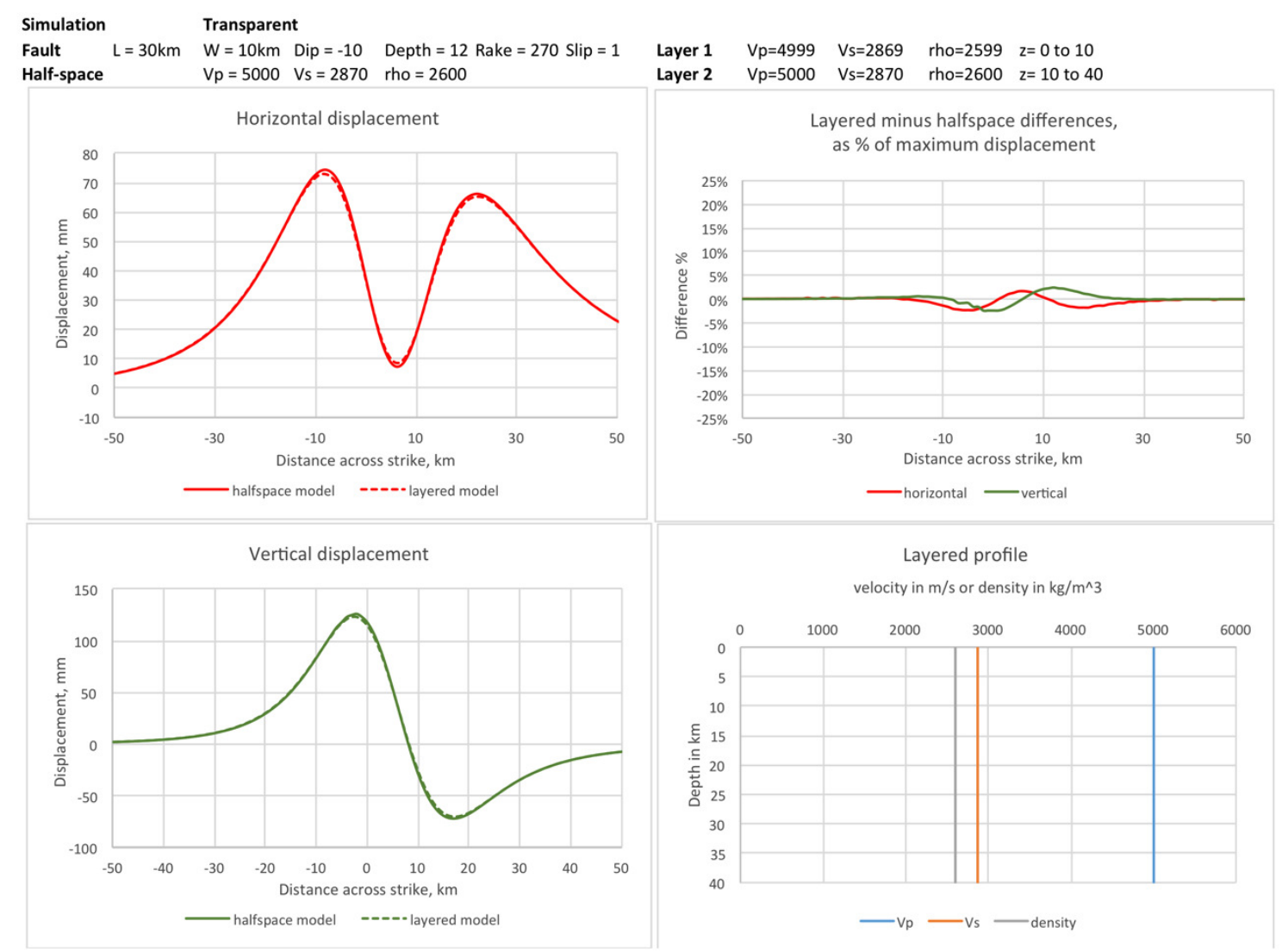

It can be seen that whilst there were some small differences at the peaks, of $2 \%$ or less, these could justifiably be ignored given the probable uncertainties in elastic parameters and layering in any practical situation.

It was therefore concluded that Smith's Matlab code and the EDGRN/EDCMP code gave results that were consistent with alternative techniques. Similar conclusions for Wang's code had been reached by Hearn and Burgmann [2005] and [Ma et al., 2012]. 


\section{Appendix 7.1: Header code to Okada DC3D.f}

c Tests Okada's finite fault routine (BSSA 82, 1992, p.1018) R Benites, GNS

$\mathrm{c}$

write $(*, *)$ 'enter velocities of $\mathrm{P}$ and $\mathrm{S}^{\prime}$

$\operatorname{read}(*, *) \mathrm{vp}, \mathrm{vs}$

write $(*, *)$ 'enter dislocation components (in meters):'

write $(*, *)$ 'strike, plunge and tensile'

$\operatorname{read}(*, *)$ DISL1,DISL2,DISL3

write $(*, *)$ 'fault parameters $(\mathrm{Km}):$ '

write $(*, *)$ 'depth_ref, length1, length2, width1, width2'

$\operatorname{read}(*, *)$ DEPTH,AL1,AL2,AW1,AW2

write $(*, *)$ 'enter dip (degrees)'

read $(*, *)$ DIP

write $(*, *)$ 'enter $\mathrm{X}, \mathrm{Y}, \mathrm{Z}(+$ down $)$ of obs. point $(\mathrm{Km})$ '

$\operatorname{read}(*, *) \mathrm{X}, \mathrm{Y}, \mathrm{Z}$

$\mathrm{c}$

$\mathrm{vsp}=\mathrm{vs} / \mathrm{vp}$

ALPHA = 1-vsp*vsp

c

call DC3D(ALPHA,X,Y,Z,DEPTH,DIP,

AL1,AL2,AW1,AW2,DISL1,DISL2,DISL3,

UX,UY,UZ,UXX,UYX,UZX,UXY,UYY,UZY,UXZ,UYZ,UZZ,IRET)

print*,'UX,UY,UZ = ', ' ' ,UX, ' ', UY, ' ', UZ

print*, '

print*, IRET

stop

end

c 


\section{Appendix 7.2: Okada test case from software manual}
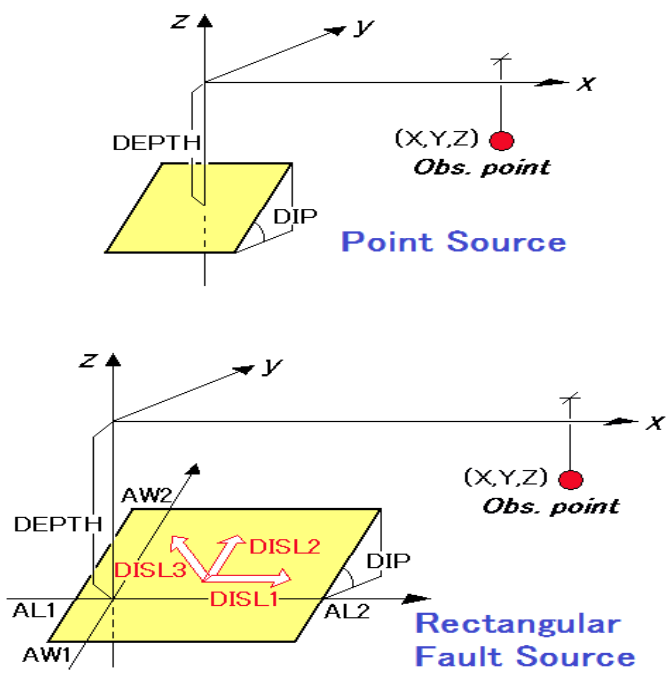

\section{Appendix-2 : Test routine to check displacement calculation by DC3D}

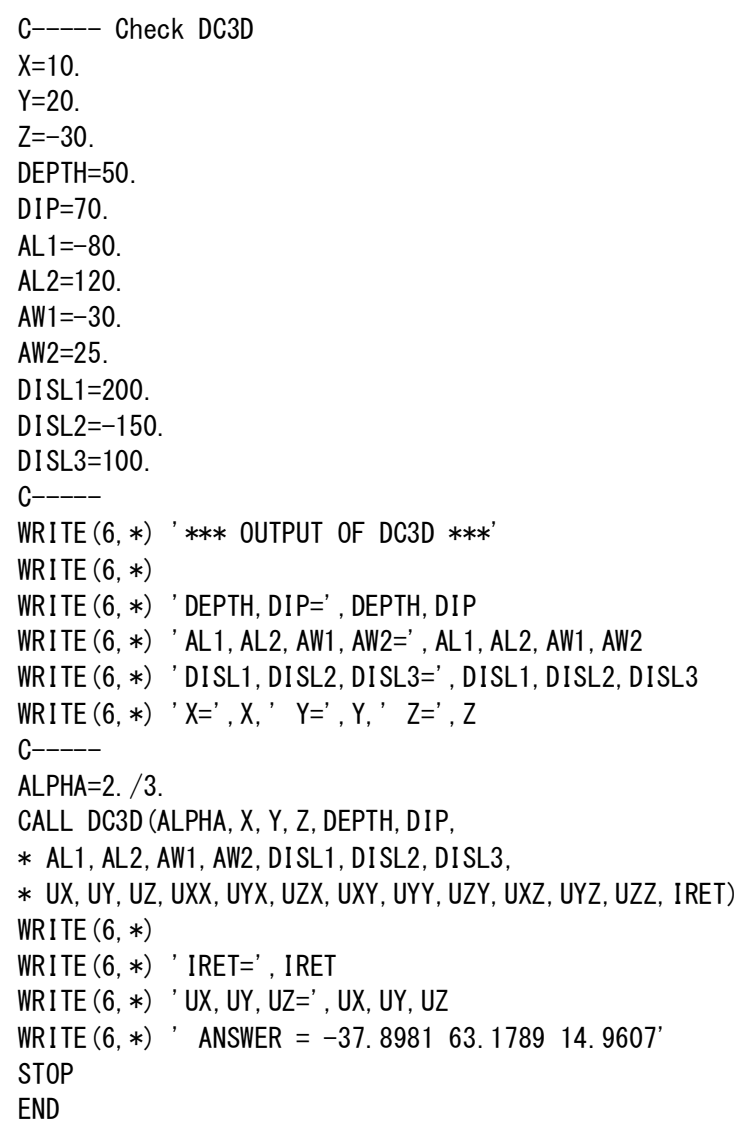




\section{Appendix 8 - Factors affecting cGPS residual noise}

The 3 components of SSE-free (or inter-SSE) residual noise rms values (as per section 3.1.2) were examined by analysis of variance to establish whether any of the known site parameters (location, elevation, type of foundation, original and final antenna model, or monument type) were likely to be contributory factors to the variation observed between sites. The 3 analyses gave similar results - an example is shown from the $\mathrm{E}$ component:

Dependent Variable: E

58 row(s) omitted due to missing values

\begin{tabular}{|c|c|c|c|c|c|}
\hline Due To & $\begin{array}{l}\text { Sum of } \\
\text { Squares }\end{array}$ & DoF & Mean Square & F-Stat & Prob \\
\hline Covariates & 12.194 & 3 & 4.065 & 8.564 & 0.0000 \\
\hline lat & 3.652 & 1 & 3.652 & 7.694 & 0.0067 \\
\hline long & 0.113 & 1 & 0.113 & 0.237 & 0.6272 \\
\hline elevation & 6.827 & 1 & 6.827 & 14.383 & 0.0003 \\
\hline Main Effects & 3.394 & 10 & 0.339 & 0.715 & 0.7084 \\
\hline Island ( $\mathbf{N}$ or $\mathrm{S}$ ) & 0.565 & 1 & 0.565 & 1.191 & 0.2779 \\
\hline Soil or Rock foundation & 0.470 & 1 & 0.470 & 0.990 & 0.3223 \\
\hline Original antenna model & 0.785 & 2 & 0.392 & 0.827 & 0.4407 \\
\hline Final antenna model & 0.249 & 1 & 0.249 & 0.525 & 0.4705 \\
\hline Monument type & 1.541 & 5 & 0.308 & 0.650 & 0.6625 \\
\hline 2 Way Interactions & 6.876 & 8 & 0.859 & 1.811 & 0.0847 \\
\hline island $x$ OAM & 0.000 & 1 & 0.000 & 0.000 & 0.9882 \\
\hline island $x$ FAM & 0.018 & 1 & 0.018 & 0.038 & 0.8451 \\
\hline island $\mathrm{x}$ monument & 0.271 & 1 & 0.271 & 0.570 & 0.4520 \\
\hline S or $R \times$ OAM & 0.661 & 1 & 0.661 & 1.393 & 0.2409 \\
\hline S or $R \times$ FAM & 0.156 & 1 & 0.156 & 0.329 & 0.5674 \\
\hline S or $\mathbf{R} \times$ monument & 1.825 & 1 & 1.825 & 3.845 & 0.0529 \\
\hline OAM x monument & 1.908 & 1 & 1.908 & 4.021 & 0.0478 \\
\hline Ant $x$ monument & 0.587 & 1 & 0.587 & 1.238 & 0.2688 \\
\hline Explained & 22.463 & 21 & 1.070 & 2.254 & 0.0043 \\
\hline Error & 44.141 & 93 & 0.475 & & \\
\hline Total & 66.604 & 114 & 0.584 & & \\
\hline
\end{tabular}

From the probability column, only Latitude and Elevation as covariates are significant effects at the 0.01 level. There is some possibility of a low level of interaction between original antenna model and monument type at the 0.05 level. 
Interactions between latitude and elevation with each component are illustrated in the following matrix, which shows that the residual noise levels in the 3 components are not strongly correlated with either latitude or elevation:

Matrix Plot

Sheet1 in Noise $7 v^{*} 115 c$
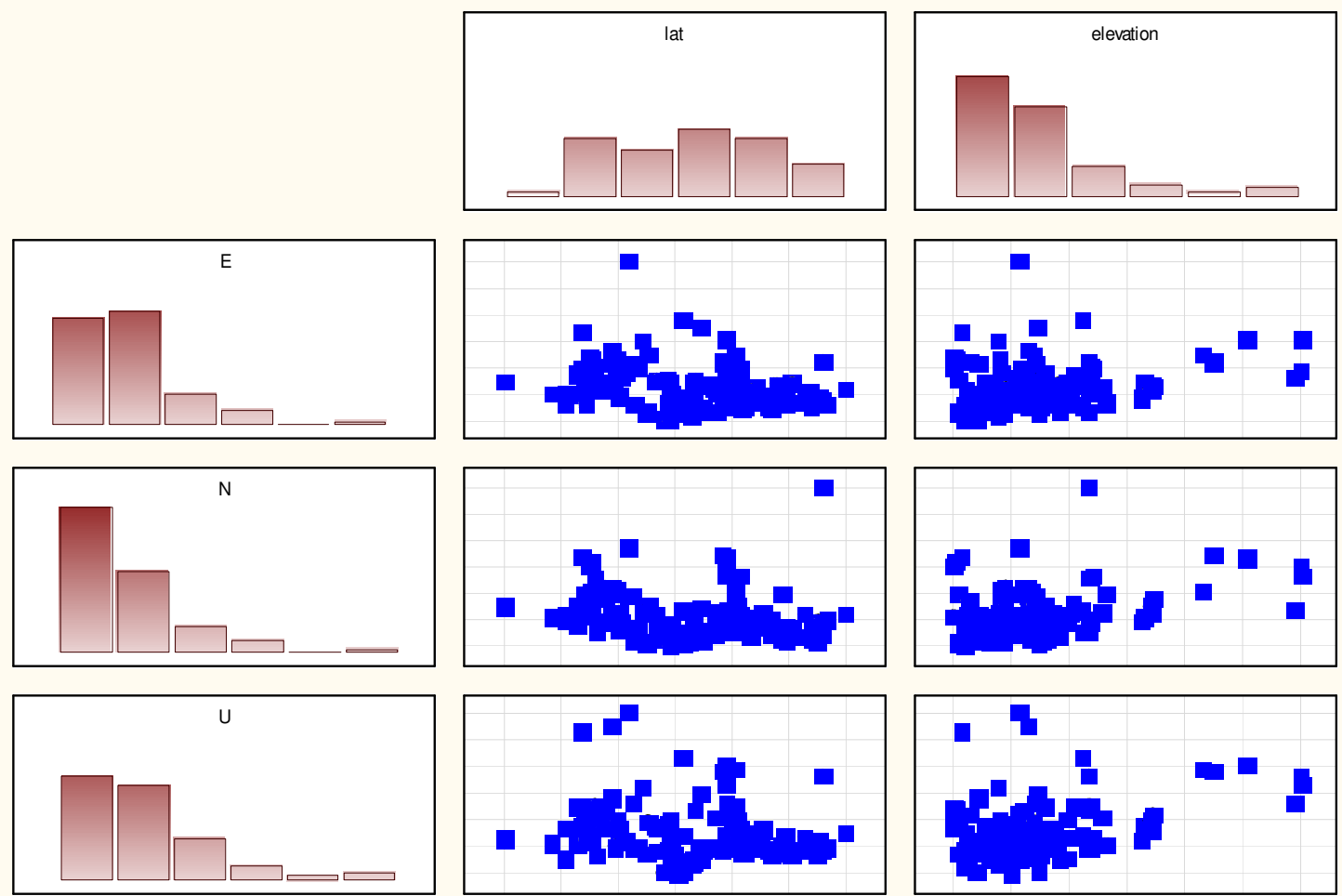

In the scatter plots, the abscissa is latitude on the left and elevation on the right, and the ordinates are, from top to bottom, the residual noise levels in the $\mathrm{E}, \mathrm{N}$ and $\mathrm{U}$ components respectively. 


\section{Appendix 9 - Evaluation of surface deformation event}

\section{categorization (ref. 3.2.1)}

Section 2.4.5 describes the 'quality' categorization ascribed to surface deformation 'events', and summary statistics are shown in section 3.2. Parameterization of 'events' was used to assess whether such categorization has utility in an objective sense. The following parameters were used to describe each 'event':

- $\mathrm{N}$ - number of cGPS sites at which the 'event' was observed

- QFS - coefficient of variation of start times of the 'event' (= standard deviation of start times between sites divided by the mean start time)

- QFE - coefficient of variation of end times of the 'event'

- $\quad c v D$ - coefficient of variation of the duration of the 'event'

- cvA - coefficient of variation of the amplitude of the event across the sites

- AbsMax - unsigned maximum amplitude of displacement over the sites at which the 'event' was observed

Linear regression was used to assess the degree of association between each of these parameters and the categorization:

\begin{tabular}{|l|c|c|c|c|c|c|}
\hline \multirow{4}{*}{$\mathrm{N}=117$} & \multicolumn{4}{|l|}{$\begin{array}{l}\text { Regression Summary for Dependent Variable: Cat } \\
\text {.52591772 } \mathrm{R}^{2}=.27658945 \text { Adjusted } \mathrm{R}^{2}=.23713069 \mathrm{~F}(6,110)=7.0096 \mathrm{p}\end{array}$} \\
\cline { 2 - 7 } & $\mathrm{b}^{*}$ & $\begin{array}{c}\text { Std. Err. } \\
\text { of } \mathrm{b}^{*}\end{array}$ & $\mathrm{~b}$ & $\begin{array}{c}\text { Std.Err. } \\
\text { of } \mathrm{b}\end{array}$ & $\mathrm{t}(110)$ & $\mathrm{p}$-value \\
\hline Intercept & & & 2.860411 & 0.233082 & 12.27212 & 0.000000 \\
\hline $\mathrm{N}$ & -0.439442 & 0.102015 & -0.090513 & 0.021012 & -4.30764 & 0.000036 \\
\hline QFS & 0.240272 & 0.093648 & 0.730832 & 0.284848 & 2.56569 & 0.011643 \\
\hline QFE & -0.102959 & 0.124424 & -0.232273 & 0.280697 & -0.82749 & 0.409753 \\
\hline cvD & 0.075678 & 0.118601 & 0.413799 & 0.648497 & 0.63809 & 0.524742 \\
\hline AbsMax & -0.168660 & 0.109362 & -0.013555 & 0.008789 & -1.54222 & 0.125891 \\
\hline CvA & 0.064264 & 0.108410 & 0.245812 & 0.414669 & 0.59279 & 0.554538 \\
\hline
\end{tabular}

$\mathrm{b}^{*}$ - standardized regression coefficient, $\mathrm{b}$ - raw regression coefficient

Significant factors are shown in red.

Whilst 150 'events' entered the analysis, 33 were only observed at a single cGPS site and therefore could not participate in the regression analysis. These events were all Cat 4 , by definition, and therefore this category is only partially represented in the outcome. Nevertheless, as indicated in the following plot of predicted versus actual categorization 
(using the above linear regression function), there is some correlation between the objective parameters and the subjective assessment:

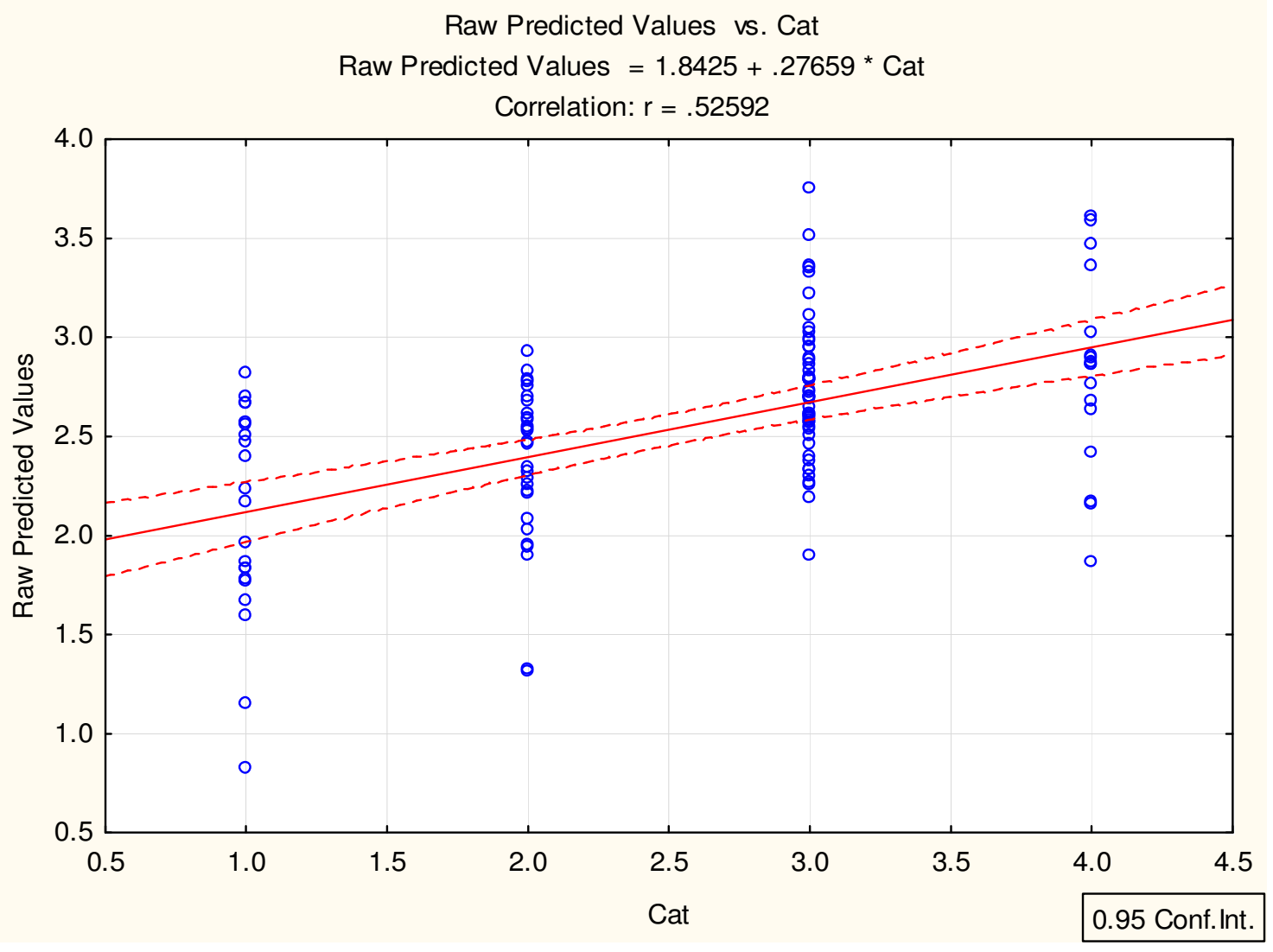

Whilst most of the Cat 4 'events' remaining in the regression would have been predicted as Cat 3, and most of the Cat 1 events would predict as Cat 2, it should be recalled that subjective factors such as shape were not taken into account in the parameterization, and also that a number of otherwise satisfactory 'events' were put into Cat 4 because they were directly associated with the Cook Strait or Lake Grasmere earthquakes. This was confirmed by outlier analysis.

An element of 'event' shape that was also examined at this stage was whether the displacement-time shape appeared to better fit a linear (ramp), exponential, or logarithmic model. Only 1 'event' was deemed a better fit to the logarithmic model and hence this model was excluded from the comparisons. The assessment was based on the stacked normalized displacement-time curves produced in 'SSEcuts' (see example Fig. 2.4.4a). Cat 4 events were also essentially excluded since they often involved single sites. The following categorization plot shows the number of events by region ( $\mathrm{sw}$ - southwest, ne - northeast, c - central, v - volcanic), category, and 'best' shape. 


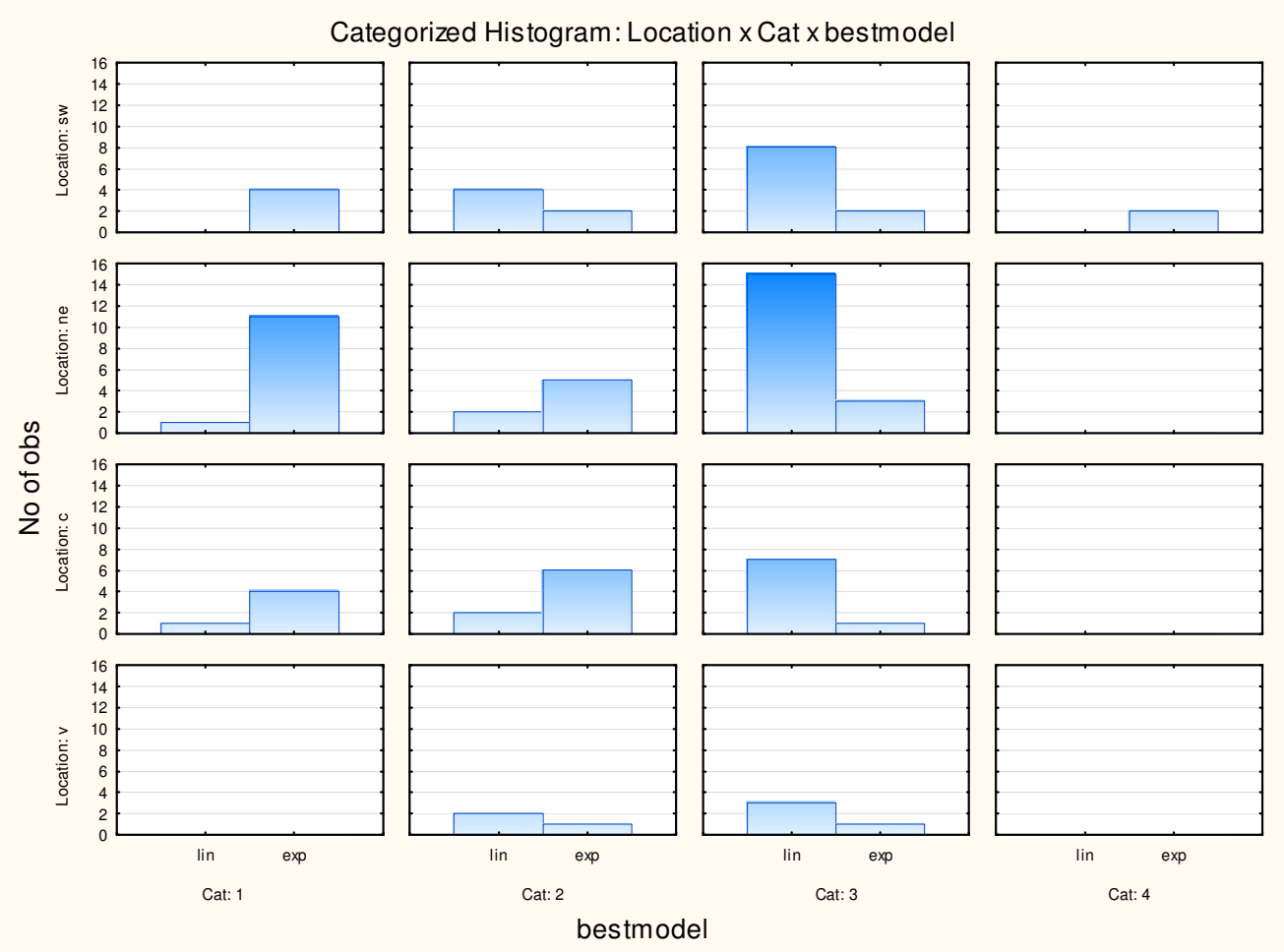

It can be seen that Cat 1 tended to contain more exponential model fits, category 2 contained similar numbers of both linear and exponential fits, and category 3 contained mostly linear fits. Since modeling (in 'SSEcuts') was limited to events with 4 or more sites, there are few category 4 events in this selection.

There was no relationship between the Category and the variable 'c' (see section 2.4.4), or the residual misfit of the exponential model, as shown in the multivariate analysis shown below:

\begin{tabular}{|l|c|c|c|c|c|c|}
\hline \multirow{4}{*}{ Effect } & \multicolumn{3}{|l}{$\begin{array}{l}\text { Multivariate Tests of } \\
\text { parameterization Effective hypothesis decomposition Exclude condition: Cat=4 OR } \\
\text { c_exp>9999 }\end{array}$} \\
\cline { 2 - 7 } & Test & Value & F & $\begin{array}{c}\text { Effect } \\
\text { df }\end{array}$ & $\begin{array}{c}\text { Error } \\
\text { df }\end{array}$ & $p$ \\
\hline Intercept & Wilks & 0.969196 & 0.69922 & 2 & 44 & 0.502408 \\
\hline QFS & Wilks & 0.951635 & 1.11811 & 2 & 44 & 0.336007 \\
\hline QFE & Wilks & 0.957403 & 0.97882 & 2 & 44 & 0.383786 \\
\hline meanD & Wilks & 0.337892 & 43.10951 & 2 & 44 & 0.000000 \\
\hline cvD & Wilks & 0.989185 & 0.24054 & 2 & 44 & 0.787230 \\
\hline AbsMax & Wilks & 0.850415 & 3.86972 & 2 & 44 & 0.028306 \\
\hline cvA & Wilks & 0.990242 & 0.21680 & 2 & 44 & 0.805947 \\
\hline Location & Wilks & 0.906989 & 0.73368 & 6 & 88 & 0.623785 \\
\hline Cat & Wilks & 0.874417 & 1.52683 & 4 & 88 & 0.201319 \\
\hline
\end{tabular}


Category 4 events were omitted for reasons already stated, and 'c' values exceeding 9999 were also excluded, since these models did not have closure (i.e. ill-constrained in either ' $c$ ' or ' $\mathrm{t}_{0}$ '). The apparent significance of the multivariate relationships with mean duration and amplitude, are, when examined by univariate analysis of covariance, simply due to univariate correlation between rms misfit and duration or amplitude, not related to the value of ' $c$ '. In other words, the time constant variable, 'c', in the exponential model, shows no apparent correlation with any of the other variables introduced here. 


\section{Appendix 10 - Example of Tablecurve 2D fit to stacked}

\section{normalized negative event 13 (ref. 3.2.5)}

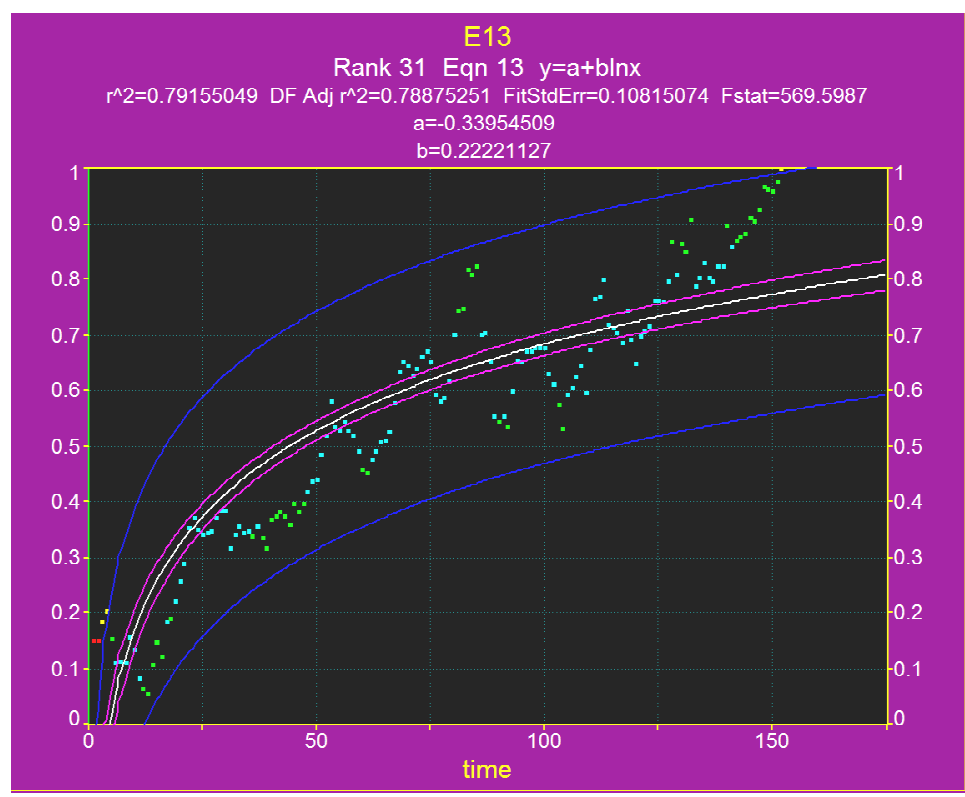

\begin{tabular}{|c|c|c|c|c|c|}
\hline Rank & $\mathbf{R}^{2}$ & FPD & Eq.\# & Equation form & (FPD = floating point demand) \\
\hline 1 & 0.9121956523 & 11 & 9 & $y=a+b x^{0.5} \ln x$ & \\
\hline 2 & 0.9118137741 & 15 & 8010 & Power $(a, b, c)$ & \\
\hline 3 & 0.9113912227 & 14 & 8156 & Power_(a,b) & \\
\hline 4 & 0.9111782642 & 10 & 73 & $\mathrm{y}^{0.5}=\mathrm{a}+\mathrm{b}(\ln \mathrm{x})^{2}$ & \\
\hline 5 & 0.9071443679 & 4 & 12 & $y=a+b x^{0.5}$ & \\
\hline 6 & 0.9067505931 & 5 & 75 & $y^{0.5}=a+b x^{0.5}$ & \\
\hline 7 & 0.9065968108 & 9 & 11 & $y=a+b x / \ln x$ & \\
\hline 8 & 0.9047115980 & 15 & 34 & $\ln y=a+b \ln x$ & \\
\hline 9 & 0.9004279931 & 16 & 31 & $\ln y=a+b(\ln x)^{2}$ & \\
\hline 10 & 0.8966943242 & 12 & 72 & $\mathrm{y}^{0.5}=\mathrm{a}+\mathrm{bx} \mathrm{x}^{0.5} \ln \mathrm{x}$ & \\
\hline 11 & 0.8962297227 & 11 & 8002 & Exponential $(a, b, c)$ & \\
\hline 12 & 0.8962295940 & 2 & 1 & $y=a+b x$ & \\
\hline 13 & 0.8925585995 & 8 & 87 & $y^{2}=a+b x^{1.5}$ & \\
\hline 14 & 0.8909429399 & 7 & 57 & $y^{-1}=a+b / x^{0.5}$ & \\
\hline 15 & 0.8901491860 & 9 & 10 & $y=a+b(\ln x)^{2}$ & \\
\hline 16 & 0.8888050700 & 9 & 76 & $\mathrm{y}^{0.5}=\mathrm{a}+\mathrm{b} \ln \mathrm{x}$ & \\
\hline 17 & 0.8817341275 & 11 & 33 & Iny $=a+b x^{0.5}$ & \\
\hline 18 & 0.8776557922 & 9 & 2 & $y=a+b x \ln x$ & \\
\hline 19 & 0.8642928008 & 3 & 64 & $y^{0.5}=a+b x$ & \\
\hline 20 & 0.8633705140 & 9 & 55 & $y^{-1}=a+b \ln x$ & \\
\hline 21 & 0.8615371245 & 18 & 30 & $\ln y=a+b x^{0.5} \ln x$ & \\
\hline 22 & 0.8461412290 & 5 & 3 & $y=a+b x^{1.5}$ & \\
\hline 23 & 0.8404941225 & 10 & 8157 & Exponential(a,b) & \\
\hline 24 & 0.8392980783 & 10 & 65 & $\mathrm{y}^{0.5}=\mathrm{a}+\mathrm{bx} \ln \mathrm{x}$ & \\
\hline 25 & 0.8304641547 & 5 & 88 & $y^{2}=a+b x^{2}$ & \\
\hline 26 & 0.8234767084 & 10 & 52 & $\mathrm{y}^{-1}=\mathrm{a}+\mathrm{b}(\ln \mathrm{x})^{2}$ & \\
\hline 27 & 0.8136232872 & 9 & 22 & $\ln y=a+b x$ & \\
\hline 28 & 0.8093902775 & 13 & 36 & $\ln y=a+b / x^{0.5}$ & \\
\hline 29 & 0.8005144401 & 7 & 66 & $y^{0.5}=a+b x^{1.5}$ & \\
\hline 30 & 0.7924630127 & 12 & 89 & $y^{2}=a+b x^{2} \ln x$ & \\
\hline 31 & 0.7915504859 & 8 & 13 & $y=a+b \ln x$ & \\
\hline
\end{tabular}




\section{Appendix 11 - Table of secular velocities (ref. 3.3)}

Full time-series cGPS velocity components referenced to AUCK, for all sites used in this study.

\begin{tabular}{|c|c|c|c|c|c|c|c|c|c|c|c|c|c|c|c|c|}
\hline \multirow{3}{*}{$\begin{array}{l}\text { Site } \\
\text { name }\end{array}$} & \multirow{3}{*}{$\begin{array}{l}\text { Long } \\
\text { degE }\end{array}$} & \multirow{3}{*}{$\begin{array}{c}\text { Lat } \\
\operatorname{degN}\end{array}$} & \multicolumn{6}{|c|}{ Overall secular velocity components } & \multicolumn{6}{|c|}{ SSE-free secular velocity components } & \multirow{2}{*}{\multicolumn{2}{|c|}{$\begin{array}{l}\text { days from } \\
\text { 01-Jan-00 }\end{array}$}} \\
\hline & & & Ve & RMSe & $\mathrm{Vn}$ & RMSn & $\mathrm{Vu}$ & RMSu & V'e & RMS'e & $V^{\prime} n$ & RMS'n & V'u & RMS'u & & \\
\hline & & & $\mathrm{mm} / \mathrm{yr}$ & $\mathrm{mm}$ & $\mathrm{mm} / \mathrm{yr}$ & $\mathrm{mm}$ & $\mathrm{mm} / \mathrm{yr}$ & $\mathrm{mm}$ & $\mathrm{mm} / \mathrm{yr}$ & $\mathrm{mm}$ & $\mathrm{mm} / \mathrm{yr}$ & $\mathrm{mm}$ & $\mathrm{mm} / \mathrm{yr}$ & $\mathrm{mm}$ & start & end \\
\hline$\overline{\mathrm{AHTI}}$ & 178.0460 & -38.4114 & -3.7 & 4.1 & -20.3 & 2.6 & 3.5 & 5.8 & -11.2 & 1.2 & -17.9 & 0.8 & 1.6 & 3.9 & 3450 & 5160 \\
\hline AKTO & 176.4612 & -40.5398 & -29.0 & 5.2 & -13.4 & 2.6 & -2.0 & 5.8 & -30.1 & 2.6 & -12.5 & 1.0 & -1.0 & 3.7 & 2309 & 5159 \\
\hline ANAU & 178.2912 & -38.2682 & -1.0 & 4.3 & -21.1 & 2.4 & -0.3 & 6.0 & -10.2 & 1.1 & -18.9 & 1.0 & -5.3 & 3.7 & 3945 & 5159 \\
\hline AVLN & 174.9329 & -41.1964 & -27.4 & 5.4 & -6.3 & 3.2 & 0.1 & 6.7 & -30.6 & 2.8 & -5.4 & 2.5 & -1.3 & 5.4 & 2247 & 5160 \\
\hline BHST & 176.0632 & -39.4892 & -6.2 & 3.5 & -7.6 & 2.7 & 3.7 & 6.3 & -8.8 & 1.9 & -9.9 & 1.0 & 0.9 & 3.8 & 3094 & 5159 \\
\hline BIRF & 176.2461 & -40.6798 & -31.5 & 3.7 & -10.3 & 2.4 & -2.9 & 5.8 & -31.4 & 2.2 & -10.0 & 1.7 & -2.9 & 3.9 & 1854 & 5159 \\
\hline BTHL & 175.1365 & -41.3405 & -36.4 & 4.4 & -8.4 & 2.8 & -1.9 & 10.2 & -35.4 & 3.7 & -8.2 & 2.5 & -2.6 & 8.6 & 3302 & 5159 \\
\hline CAST & 176.2016 & -40.9098 & -31.6 & 4.0 & -11.1 & 1.9 & -1.9 & 7.3 & -32.1 & 2.5 & -10.5 & 1.4 & -2.2 & 6.0 & 2233 & 5159 \\
\hline CKID & 177.0764 & -39.6579 & -10.3 & 4.4 & -17.9 & 3.0 & 2.8 & 5.9 & -13.6 & 1.6 & -15.4 & 1.0 & 3.9 & 3.1 & 2336 & 5159 \\
\hline CLIM & 175.1455 & -41.1447 & -29.4 & 5.0 & -7.9 & 3.0 & -0.7 & 7.3 & -32.0 & 2.6 & -6.0 & 2.2 & -1.6 & 5.5 & 2119 & 5160 \\
\hline CMBL & 174.2138 & -41.7490 & -36.3 & 50.2 & -7.7 & 4.7 & -0.1 & 17.1 & -29.9 & 2.0 & -6.4 & 1.5 & 0.1 & 3.9 & 1489 & 5159 \\
\hline CNST & 178.2111 & -38.4880 & -6.4 & 6.5 & -20.9 & 3.1 & 1.7 & 5.8 & -20.8 & 1.9 & -15.3 & 0.9 & 3.3 & 3.4 & 2845 & 5159 \\
\hline DNVK & 176.1667 & -40.2989 & -20.9 & 6.5 & -11.1 & 3.1 & -1.5 & 7.5 & -23.7 & 2.1 & -9.0 & 1.3 & -3.2 & 4.5 & 1014 & 5160 \\
\hline DURV & 173.9216 & -40.8018 & -11.5 & 6.2 & 0.6 & 2.3 & -0.1 & 6.8 & -15.0 & 2.2 & 1.4 & 1.4 & 1.8 & 4.1 & 1896 & 5160 \\
\hline FRTN & 177.4099 & -38.9393 & -3.9 & 3.2 & -19.7 & 1.9 & 1.3 & 6.1 & -9.3 & 1.3 & -17.3 & 1.0 & 2.3 & 4.4 & 4037 & 5160 \\
\hline GISB & 177.8860 & -38.6353 & -4.3 & 7.0 & -19.7 & 3.8 & 0.4 & 6.3 & -14.4 & 1.2 & -15.9 & 1.0 & 5.0 & 3.7 & 910 & 5160 \\
\hline GLDB & 172.5296 & -40.8266 & -6.6 & 3.7 & 3.1 & 2.3 & 0.2 & 6.8 & -6.5 & 2.6 & 3.1 & 1.9 & -0.1 & 4.9 & 1494 & 5160 \\
\hline GNBK & 175.2381 & -40.0803 & -11.1 & 3.9 & -3.9 & 3.2 & 0.0 & 5.6 & -13.1 & 2.0 & -4.1 & 0.9 & -0.3 & 2.8 & 3009 & 5160 \\
\hline GRAC & 174.9170 & -41.2351 & -28.8 & 4.2 & -5.8 & 3.1 & -2.8 & 7.5 & -29.9 & 3.2 & -6.0 & 2.7 & -4.1 & 5.4 & 26 & 2431 \\
\hline GRNG & 175.4593 & -39.9763 & -10.6 & 4.4 & -5.1 & 2.7 & 0.9 & 5.5 & -13.6 & 1.8 & -4.1 & 1.3 & 0.5 & 2.6 & 2730 & 5160 \\
\hline HANA & 177.5694 & -38.6868 & -2.7 & 3.9 & -18.4 & 2.1 & 2.9 & 5.5 & -5.4 & 1.1 & -18.2 & 1.2 & 4.2 & 3.5 & 2777 & 5159 \\
\hline HAST & 176.7266 & -39.6170 & -9.7 & 5.1 & -14.4 & 2.5 & -2.1 & 6.3 & -15.4 & 1.6 & -13.6 & 0.9 & -5.5 & 3.7 & 993 & 5159 \\
\hline HIKB & 178.3034 & -37.5610 & 2.1 & 3.4 & -19.3 & 2.0 & 1.3 & 6.3 & 1.1 & 1.7 & -19.9 & 1.5 & 1.2 & 4.4 & 1233 & 5159 \\
\hline HOLD & 175.5152 & -40.8972 & -27.9 & 5.5 & -9.0 & 2.3 & -1.1 & 10.3 & -29.3 & 3.6 & -8.9 & 1.8 & -2.4 & 9.1 & 2632 & 5156 \\
\hline KAHU & 176.8763 & -39.7938 & -14.5 & 4.8 & -14.3 & 2.7 & 0.9 & 6.0 & -19.2 & 1.6 & -12.4 & 0.9 & 2.0 & 3.1 & 2302 & 5160 \\
\hline KAIK & 173.5337 & -42.4255 & -34.2 & 3.1 & -7.7 & 2.5 & -0.1 & 6.7 & -34.4 & 1.8 & -8.1 & 1.7 & -0.1 & 4.2 & 1493 & 5160 \\
\hline KAPT & 174.9098 & -40.8609 & -19.0 & 7.7 & -3.0 & 3.7 & 0.9 & 7.5 & -23.9 & 2.1 & -4.3 & 1.8 & -3.4 & 3.7 & 1623 & 5160 \\
\hline
\end{tabular}




\begin{tabular}{|c|c|c|c|c|c|c|c|c|c|c|c|c|c|c|c|c|}
\hline \multirow{3}{*}{$\begin{array}{l}\text { Site } \\
\text { name }\end{array}$} & \multirow{3}{*}{$\begin{array}{l}\text { Long } \\
\text { degE }\end{array}$} & \multirow{3}{*}{$\begin{array}{c}\text { Lat } \\
\operatorname{degN}\end{array}$} & \multicolumn{6}{|c|}{ Overall secular velocity components } & \multicolumn{6}{|c|}{ SSE-free secular velocity components } & \multirow{2}{*}{\multicolumn{2}{|c|}{$\begin{array}{l}\text { days from } \\
\text { 01-Jan-00 }\end{array}$}} \\
\hline & & & Ve & RMSe & $\mathrm{Vn}$ & RMSn & $\mathrm{Vu}$ & RMSu & V'e & RMS'e & $V^{\prime} n$ & RMS'n & $\mathrm{V}^{\prime} \mathrm{u}$ & RMS'u & & \\
\hline & & & $\mathrm{mm} / \mathrm{yr}$ & $\mathrm{mm}$ & $\mathrm{mm} / \mathrm{yr}$ & $\mathrm{mm}$ & $\mathrm{mm} / \mathrm{yr}$ & $\mathrm{mm}$ & $\mathrm{mm} / \mathrm{yr}$ & $\mathrm{mm}$ & $\mathrm{mm} / \mathrm{yr}$ & $\mathrm{mm}$ & $\mathrm{mm} / \mathrm{yr}$ & $\mathrm{mm}$ & start & end \\
\hline KAWK & 176.4228 & -39.4240 & -5.4 & 3.5 & -13.8 & 3.4 & 0.7 & 7.5 & -5.8 & 1.6 & -15.3 & 1.4 & 0.1 & 3.9 & 3806 & 5160 \\
\hline KERE & 176.3701 & -39.6432 & -8.1 & 6.7 & -12.0 & 4.0 & 0.5 & 10.8 & -12.7 & 3.8 & -14.8 & 1.9 & -5.1 & 6.4 & 2330 & 5160 \\
\hline КОКО & 177.6678 & -39.0161 & -5.2 & 5.5 & -22.0 & 2.2 & 0.9 & 5.6 & -14.5 & 1.1 & -19.4 & 1.0 & 3.9 & 3.2 & 1770 & 5160 \\
\hline KORO & 175.4241 & -40.4093 & -19.8 & 6.0 & -4.6 & 4.0 & -1.6 & 6.8 & -19.4 & 1.6 & -6.1 & 0.9 & -3.8 & 3.4 & 2728 & 5160 \\
\hline KUTA & 177.0698 & -39.1723 & -4.6 & 3.2 & -17.9 & 2.5 & 1.1 & 6.3 & -9.8 & 1.2 & -15.2 & 1.2 & -0.2 & 4.0 & 3750 & 5160 \\
\hline LEVN & 175.2406 & -40.5888 & -21.0 & 8.2 & -4.4 & 3.7 & 0.3 & 8.2 & -26.3 & 1.9 & -5.2 & 0.8 & -3.9 & 3.6 & 3133 & 5159 \\
\hline LEYL & 176.9367 & -39.3323 & -5.4 & 3.1 & -17.7 & 2.1 & -1.2 & 6.2 & -9.1 & 2.1 & -17.2 & 1.1 & -2.5 & 3.5 & 2717 & 5158 \\
\hline MAHA & 173.7938 & -41.2914 & -13.1 & 8.3 & -0.9 & 2.0 & 0.4 & 6.6 & -18.7 & 1.9 & -0.4 & 1.5 & 2.5 & 3.9 & 3608 & 5156 \\
\hline MAHI & 177.9070 & -39.1526 & -6.9 & 8.9 & -21.8 & 3.7 & 2.6 & 6.0 & -25.1 & 1.5 & -14.3 & 1.0 & 1.7 & 3.8 & 2774 & 5159 \\
\hline MAKO & 178.1291 & -38.6438 & -12.9 & 5.1 & -18.0 & 1.9 & 2.7 & 6.0 & -24.6 & 1.0 & -15.4 & 1.0 & 4.4 & 3.4 & 3967 & 5160 \\
\hline MANG & 175.5749 & -40.6687 & -25.9 & 7.7 & -6.6 & 3.2 & -1.0 & 10.2 & -27.0 & 5.8 & -6.9 & 2.9 & -2.1 & 8.9 & 1870 & 5157 \\
\hline MAST & 175.5846 & -41.0620 & -31.9 & 3.3 & -10.0 & 1.8 & -2.4 & 6.8 & -31.7 & 1.8 & -10.1 & 1.4 & -2.4 & 4.7 & 1100 & 5159 \\
\hline MATW & 177.5262 & -38.3338 & -1.6 & 3.4 & -17.5 & 1.8 & 2.9 & 5.8 & -1.6 & 1.9 & -17.5 & 1.2 & 3.1 & 3.8 & 1561 & 5157 \\
\hline MCNL & 176.6965 & -39.4442 & -5.7 & 3.7 & -15.3 & 2.6 & 0.3 & 6.8 & -9.6 & 2.1 & -16.0 & 1.0 & -5.3 & 3.8 & 3070 & 5157 \\
\hline MING & 176.7497 & -38.6169 & 0.1 & 2.8 & -13.1 & 1.7 & 2.3 & 5.1 & 0.2 & 1.5 & -13.5 & 1.4 & 3.3 & 3.6 & 3771 & 5157 \\
\hline MKNO & 176.0288 & -39.7034 & -12.8 & 3.3 & -11.1 & 1.8 & -3.3 & 6.9 & -13.2 & 1.9 & -11.3 & 1.2 & -2.8 & 5.3 & 4154 & 5158 \\
\hline MNHR & 176.2234 & -40.4686 & -26.4 & 4.7 & -10.8 & 2.3 & -4.1 & 9.3 & -27.8 & 3.4 & -10.8 & 1.6 & -3.1 & 6.8 & 4080 & 5160 \\
\hline MTBL & 175.5362 & -40.1814 & -15.2 & 3.9 & -6.6 & 3.5 & -1.1 & 5.8 & -16.1 & 2.0 & -7.0 & 1.0 & -4.0 & 2.6 & 3012 & 5160 \\
\hline MTQN & 175.2414 & -41.0016 & -26.0 & 5.6 & -7.3 & 2.2 & -0.2 & 7.3 & -30.0 & 1.7 & -6.1 & 1.6 & -2.1 & 5.9 & 3693 & 5156 \\
\hline NLSN & 173.4337 & -41.1835 & -10.8 & 5.6 & 1.4 & 2.1 & -0.4 & 6.3 & -13.8 & 1.9 & 1.9 & 1.2 & 0.7 & 3.9 & 1501 & 5159 \\
\hline NMAI & 176.8066 & -39.0970 & -2.6 & 3.6 & -15.8 & 3.1 & 3.1 & 6.4 & -4.5 & 2.6 & -18.3 & 2.3 & 0.4 & 4.2 & 3020 & 5159 \\
\hline NPLY & 174.1182 & -39.1826 & -4.4 & 3.5 & 0.5 & 1.9 & 1.0 & 7.6 & -4.6 & 2.2 & 0.6 & 1.5 & 0.9 & 5.4 & 1176 & 5159 \\
\hline NRRD & 175.7613 & -40.3854 & -23.0 & 5.6 & -8.0 & 3.6 & -2.4 & 6.5 & -23.1 & 3.1 & -7.4 & 1.6 & -7.3 & 4.1 & 3009 & 5159 \\
\hline NRSW & 176.2000 & -40.1133 & -19.2 & 3.8 & -7.6 & 1.9 & -4.6 & 7.7 & -21.2 & 1.9 & -7.7 & 1.2 & -5.2 & 5.0 & 4036 & 5160 \\
\hline OHIN & 175.7907 & -39.9183 & -10.3 & 5.1 & -8.0 & 2.1 & 3.3 & 7.4 & -15.1 & 1.7 & -9.6 & 0.8 & -2.7 & 2.8 & 3169 & 5160 \\
\hline $\mathrm{OKOH}$ & 174.0603 & -41.0193 & -14.9 & 6.4 & 1.8 & 4.7 & 0.5 & 6.4 & -19.8 & 1.9 & -0.8 & 1.6 & 2.3 & 4.3 & 3608 & 5160 \\
\hline OPTK & 177.3076 & -38.0465 & -0.9 & 3.2 & -15.2 & 1.6 & 0.8 & 5.6 & -1.5 & 1.3 & -15.3 & 0.9 & 2.4 & 3.5 & 3520 & 5160 \\
\hline OROA & 176.6807 & -40.1044 & -17.6 & 3.9 & -14.3 & 2.5 & 0.4 & 6.0 & -22.9 & 1.3 & -12.9 & 0.9 & 2.7 & 3.4 & 3965 & 5160 \\
\hline OTAK & 175.1704 & -40.8165 & -23.4 & 7.9 & -4.9 & 2.0 & 0.3 & 8.1 & -26.2 & 1.7 & -5.8 & 1.1 & -2.0 & 4.2 & 2838 & 5160 \\
\hline PAEK & 174.9521 & -41.0218 & -22.8 & 6.0 & -4.4 & 3.0 & 2.2 & 8.9 & -26.9 & 1.8 & -4.5 & 2.0 & -2.6 & 5.3 & 102 & 5159 \\
\hline
\end{tabular}




\begin{tabular}{|c|c|c|c|c|c|c|c|c|c|c|c|c|c|c|c|c|}
\hline \multirow{3}{*}{$\begin{array}{l}\text { Site } \\
\text { name }\end{array}$} & \multirow{3}{*}{$\begin{array}{l}\text { Long } \\
\text { degE }\end{array}$} & \multirow{3}{*}{$\begin{array}{c}\text { Lat } \\
\operatorname{deg} N\end{array}$} & \multicolumn{6}{|c|}{ Overall secular velocity components } & \multicolumn{6}{|c|}{ SSE-free secular velocity components } & \multirow{2}{*}{\multicolumn{2}{|c|}{$\begin{array}{l}\text { days from } \\
\text { 01-Jan-00 }\end{array}$}} \\
\hline & & & $\mathrm{Ve}$ & RMSe & $\mathrm{Vn}$ & RMSn & $\mathrm{Vu}$ & RMSu & V'e & RMS'e & $V^{\prime} n$ & RMS'n & V'u & RMS'u & & \\
\hline & & & $\mathrm{mm} / \mathrm{yr}$ & $\mathrm{mm}$ & $\mathrm{mm} / \mathrm{yr}$ & $\mathrm{mm}$ & $\mathrm{mm} / \mathrm{yr}$ & $\mathrm{mm}$ & $\mathrm{mm} / \mathrm{yr}$ & $\mathrm{mm}$ & $\mathrm{mm} / \mathrm{yr}$ & $\mathrm{mm}$ & $\mathrm{mm} / \mathrm{yr}$ & $\mathrm{mm}$ & start & end \\
\hline PAKI & 178.0826 & -37.8940 & 1.5 & 4.2 & -16.9 & 4.9 & 3.9 & 8.5 & -4.1 & 2.4 & -21.2 & 4.3 & 3.3 & 6.8 & 3900 & 5159 \\
\hline PALI & 175.2548 & -41.5692 & -38.9 & 3.5 & -8.9 & 2.0 & -2.2 & 6.3 & -38.9 & 1.9 & -8.5 & 1.4 & -2.2 & 4.6 & 2638 & 5156 \\
\hline PARI & 177.8833 & -38.9226 & -1.4 & 11.1 & -21.7 & 3.1 & -0.5 & 6.5 & -20.4 & 1.8 & -15.5 & 0.9 & 1.2 & 3.8 & 2771 & 5160 \\
\hline PARW & 175.4269 & -41.3815 & -38.0 & 3.5 & -9.0 & 2.1 & -2.7 & 7.0 & -38.4 & 2.3 & -8.3 & 1.5 & -3.5 & 5.3 & 1658 & 5160 \\
\hline PAWA & 176.8639 & -40.0331 & -15.6 & 8.4 & -16.9 & 4.2 & 1.5 & 7.5 & -22.9 & 1.5 & -14.0 & 1.4 & 5.1 & 3.7 & 1910 & 5160 \\
\hline PKNO & 175.1819 & -39.8048 & -11.1 & 2.9 & -0.4 & 2.2 & 3.8 & 6.1 & -11.1 & 0.8 & -1.8 & 1.1 & 3.1 & 2.8 & 4080 & 5159 \\
\hline PNUI & 176.2005 & -39.9168 & -12.5 & 8.5 & -11.3 & 2.4 & 0.3 & 14.1 & -18.7 & 4.3 & -10.6 & 1.5 & -8.0 & 8.6 & 2373 & 5156 \\
\hline PORA & 176.6352 & -40.2664 & -21.4 & 6.3 & -15.3 & 3.1 & -0.4 & 6.3 & -24.9 & 2.4 & -13.3 & 1.2 & -1.8 & 3.5 & 2276 & 5159 \\
\hline PRTU & 177.6979 & -38.8142 & -4.5 & 6.6 & -18.9 & 2.6 & 4.3 & 6.1 & -14.0 & 1.3 & -18.4 & 1.6 & 5.6 & 3.8 & 3518 & 5159 \\
\hline PTOI & 175.9993 & -40.6011 & -29.5 & 4.2 & -10.4 & 2.6 & -2.4 & 6.6 & -28.7 & 2.5 & -9.9 & 1.8 & -2.9 & 5.6 & 2273 & 5160 \\
\hline PUKE & 178.2574 & -38.0714 & 2.7 & 4.2 & -19.8 & 1.9 & 3.0 & 5.7 & -3.5 & 1.1 & -19.6 & 1.0 & 5.5 & 4.0 & 1508 & 5160 \\
\hline RAHI & 177.0861 & -38.9162 & -2.0 & 3.2 & -16.9 & 2.1 & 1.4 & 6.6 & -4.2 & 1.8 & -15.5 & 1.5 & -0.7 & 4.2 & 3062 & 5160 \\
\hline RAKW & 176.6212 & -39.7472 & -14.9 & 3.1 & -14.5 & 1.8 & -4.6 & 6.3 & -17.5 & 1.1 & -13.7 & 1.0 & -2.4 & 3.4 & 4080 & 5156 \\
\hline RAUM & 177.6775 & -37.9650 & 0.4 & 3.1 & -17.5 & 1.8 & 2.5 & 5.7 & -0.1 & 1.3 & -18.2 & 1.3 & 4.8 & 3.8 & 4157 & 5157 \\
\hline RAWI & 177.4154 & -38.4956 & -2.4 & 3.4 & -17.4 & 2.5 & 2.9 & 5.7 & -2.4 & 1.2 & -17.0 & 2.0 & 4.1 & 4.0 & 3449 & 5156 \\
\hline RDLV & 175.4043 & -41.1869 & -35.4 & 3.7 & -9.6 & 2.3 & -2.2 & 7.0 & -34.8 & 2.3 & -9.3 & 1.7 & -3.0 & 4.9 & 3302 & 5157 \\
\hline RGAR & 176.3430 & -38.5620 & -2.9 & 3.3 & -6.6 & 1.8 & -0.8 & 5.6 & -1.3 & 2.0 & -7.5 & 1.0 & -0.6 & 3.6 & 2868 & 5157 \\
\hline RGAW & 176.8962 & -38.0032 & 0.4 & 3.0 & -13.4 & 1.7 & 2.3 & 5.6 & 2.7 & 1.3 & -13.8 & 1.1 & 2.1 & 3.3 & 3371 & 5160 \\
\hline RGKW & 176.7728 & -38.0525 & -2.6 & 3.0 & -12.1 & 1.7 & 0.9 & 5.8 & -0.2 & 1.6 & -11.7 & 1.2 & -0.1 & 3.9 & 3255 & 5159 \\
\hline RGMT & 176.7247 & -37.9155 & -1.2 & 3.3 & -7.8 & 3.2 & 8.0 & 6.6 & 0.3 & 1.4 & -7.8 & 1.5 & 9.7 & 3.9 & 2860 & 5157 \\
\hline RGRR & 176.5146 & -38.3389 & -3.0 & 3.2 & -7.1 & 1.7 & -1.8 & 5.8 & -1.1 & 2.0 & -7.7 & 1.0 & -0.5 & 3.4 & 2905 & 5159 \\
\hline RIPA & 176.4925 & -39.1655 & -2.8 & 3.7 & -12.0 & 3.0 & 2.4 & 7.8 & -3.4 & 2.6 & -13.1 & 2.0 & 1.3 & 5.7 & 1514 & 5158 \\
\hline SNST & 177.3475 & -38.7796 & -2.3 & 3.8 & -18.3 & 2.2 & 2.6 & 6.6 & -2.7 & 1.7 & -18.7 & 1.6 & 2.3 & 4.8 & 3447 & 5158 \\
\hline TAKP & 175.9629 & -40.0616 & -14.8 & 8.8 & -9.6 & 3.7 & -0.1 & 11.2 & -19.8 & 1.9 & -8.7 & 1.2 & -6.1 & 5.1 & 1492 & 5156 \\
\hline TAUW & 178.0059 & -38.1624 & -2.9 & 3.4 & -19.7 & 2.0 & 5.0 & 6.1 & -10.5 & 1.6 & -14.8 & 1.3 & 4.7 & 4.1 & 3519 & 5158 \\
\hline TEMA & 175.8905 & -41.1066 & -33.5 & 3.1 & -11.0 & 1.6 & -1.6 & 5.5 & -32.8 & 1.7 & -10.6 & 1.0 & -1.6 & 3.3 & 2634 & 5158 \\
\hline TGRA & 175.7701 & -38.8634 & -5.6 & 3.3 & -2.4 & 1.8 & 2.8 & 6.2 & -4.6 & 1.9 & -2.5 & 1.3 & 2.3 & 4.1 & 2294 & 5156 \\
\hline TGRI & 175.8585 & -38.9771 & -2.9 & 2.9 & -4.9 & 1.7 & 0.7 & 6.1 & -3.0 & 1.6 & -5.3 & 1.1 & 1.2 & 3.9 & 4112 & 5159 \\
\hline THAP & 175.7856 & -39.6825 & -7.3 & 4.6 & -6.4 & 1.8 & 3.9 & 6.6 & -10.7 & 1.7 & -7.2 & 1.0 & 1.0 & 3.7 & 3094 & 5158 \\
\hline TINT & 175.8857 & -40.7760 & -30.5 & 3.7 & -9.7 & 1.8 & -3.4 & 6.0 & -30.1 & 1.8 & -9.3 & 1.2 & -4.0 & 3.7 & 2288 & 5156 \\
\hline TKAR & 177.8114 & -38.4375 & -3.5 & 3.4 & -19.4 & 1.9 & 1.3 & 6.0 & -9.1 & 1.5 & -18.9 & 1.1 & 0.7 & 4.6 & 3807 & 5159 \\
\hline TORY & 174.2801 & -41.1916 & -17.9 & 6.9 & 0.0 & 8.8 & 3.3 & 11.9 & -19.9 & 2.0 & -1.5 & 1.9 & -0.4 & 5.1 & 1938 & 5156 \\
\hline
\end{tabular}




\begin{tabular}{|c|c|c|c|c|c|c|c|c|c|c|c|c|c|c|c|c|}
\hline \multirow{3}{*}{$\begin{array}{l}\text { Site } \\
\text { name }\end{array}$} & \multirow{3}{*}{$\begin{array}{l}\text { Long } \\
\text { degE }\end{array}$} & \multirow{3}{*}{$\begin{array}{c}\text { Lat } \\
\operatorname{degN}\end{array}$} & \multicolumn{6}{|c|}{ Overall secular velocity components } & \multicolumn{6}{|c|}{ SSE-free secular velocity components } & \multirow{2}{*}{\multicolumn{2}{|c|}{$\begin{array}{l}\text { days from } \\
\text { 01-Jan-00 }\end{array}$}} \\
\hline & & & $\mathrm{Ve}$ & RMSe & $\mathrm{Vn}$ & RMSn & $\mathrm{Vu}$ & RMSu & V'e & RMS'e & $V^{\prime} n$ & RMS'n & V'u & RMS'u & & \\
\hline & & & $\mathrm{mm} / \mathrm{yr}$ & $\mathrm{mm}$ & $\mathrm{mm} / \mathrm{yr}$ & $\mathrm{mm}$ & $\mathrm{mm} / \mathrm{yr}$ & $\mathrm{mm}$ & $\mathrm{mm} / \mathrm{yr}$ & $\mathrm{mm}$ & $\mathrm{mm} / \mathrm{yr}$ & $\mathrm{mm}$ & $\mathrm{mm} / \mathrm{yr}$ & $\mathrm{mm}$ & start & end \\
\hline TRAV & 175.6879 & -41.3980 & -38.5 & 3.2 & -9.5 & 1.8 & -2.6 & 6.2 & -38.8 & 1.6 & -9.1 & 1.2 & -2.1 & 3.9 & 2001 & 5159 \\
\hline TRWH & 174.6276 & -41.2781 & -20.3 & 7.1 & 3.9 & 8.1 & -0.8 & 6.6 & -24.7 & 1.2 & -4.3 & 1.7 & -2.4 & 4.1 & 3989 & 5158 \\
\hline TURI & 176.3826 & -40.2650 & -22.9 & 3.5 & -10.0 & 1.7 & -0.8 & 6.6 & -23.2 & 1.9 & -10.6 & 1.1 & -0.3 & 4.5 & 4262 & 5156 \\
\hline UTKU & 175.8314 & -39.7454 & -8.0 & 5.1 & -7.0 & 2.0 & 4.2 & 7.1 & -12.2 & 1.9 & -8.9 & 0.9 & -0.1 & 3.3 & 3241 & 5157 \\
\hline VGFW & 175.5525 & -39.2550 & -4.1 & 3.6 & -2.1 & 2.3 & 1.3 & 7.4 & -4.8 & 2.1 & -1.3 & 1.5 & 2.0 & 6.2 & 1541 & 5160 \\
\hline VGKR & 175.6413 & -39.0944 & -3.5 & 3.3 & -3.6 & 1.9 & -0.1 & 6.2 & -2.7 & 2.1 & -3.6 & 1.6 & -0.4 & 4.3 & 1888 & 5159 \\
\hline VGMO & 175.7543 & -39.4074 & -5.7 & 3.2 & -4.6 & 2.2 & 2.3 & 6.8 & -6.1 & 1.8 & -4.7 & 1.4 & 3.3 & 5.1 & 1917 & 5160 \\
\hline VGMT & 175.4705 & -39.3846 & -5.2 & 3.5 & -2.3 & 2.5 & 2.3 & 5.9 & -6.1 & 1.4 & -1.2 & 1.2 & 3.4 & 3.6 & 1859 & 5159 \\
\hline VGOB & 175.5422 & -39.1998 & -3.7 & 3.4 & -2.0 & 2.0 & 0.5 & 6.5 & -3.4 & 2.0 & -1.6 & 1.3 & 1.0 & 4.7 & 1503 & 5160 \\
\hline VGOT & 175.6651 & -39.1631 & -4.4 & 3.7 & -2.8 & 2.5 & -0.4 & 7.7 & -3.7 & 3.0 & -2.6 & 2.0 & -0.5 & 6.5 & 2352 & 5160 \\
\hline VGPK & 175.3464 & -39.2893 & -5.0 & 3.1 & -1.8 & 2.5 & 1.6 & 6.2 & -5.4 & 1.5 & -0.7 & 1.0 & 2.7 & 4.3 & 1848 & 5160 \\
\hline VGTR & 175.5483 & -39.2984 & -4.9 & 3.8 & -2.3 & 3.3 & 1.5 & 9.9 & -4.8 & 2.5 & -1.7 & 2.4 & 2.5 & 7.6 & 1869 & 5157 \\
\hline VGTS & 175.6089 & -39.2773 & -4.8 & 4.6 & -2.6 & 3.2 & 1.2 & 9.0 & -4.8 & 3.7 & -2.2 & 2.7 & 2.6 & 7.4 & 1903 & 5160 \\
\hline VGWH & 175.5890 & -39.2824 & -4.6 & 4.4 & -2.5 & 3.0 & 1.1 & 8.4 & -4.4 & 3.5 & -1.9 & 2.4 & 2.7 & 6.8 & 1883 & 5158 \\
\hline VGWN & 175.5979 & -39.3269 & -4.8 & 4.1 & -2.8 & 3.2 & 2.2 & 8.6 & -4.4 & 2.8 & -2.4 & 2.5 & 2.3 & 7.1 & 1600 & 5160 \\
\hline VGWT & 175.5897 & -39.1151 & -1.8 & 2.9 & -1.7 & 1.8 & -0.2 & 6.1 & -2.8 & 1.6 & -2.4 & 1.5 & 0.7 & 4.9 & 4333 & 5158 \\
\hline WAHU & 177.2344 & -39.0772 & -4.0 & 3.5 & -18.4 & 2.6 & 1.2 & 5.6 & -8.6 & 1.1 & -14.3 & 1.1 & 1.5 & 3.6 & 3447 & 5160 \\
\hline WANG & 174.8214 & -39.7869 & -7.0 & 5.3 & -0.9 & 2.3 & 1.4 & 5.4 & -9.7 & 1.5 & 0.3 & 0.9 & 2.1 & 3.0 & 1204 & 5158 \\
\hline WGTN & 174.8059 & -41.3235 & -29.0 & 4.0 & -6.3 & 2.9 & -0.7 & 6.7 & -30.1 & 2.7 & -5.8 & 1.4 & -2.0 & 4.8 & 2 & 5156 \\
\hline WGTT & 174.7816 & -41.2904 & -27.2 & 4.3 & -5.4 & 3.9 & -1.0 & 7.6 & -28.2 & 2.3 & -5.5 & 1.9 & -3.1 & 4.8 & 6 & 5158 \\
\hline WHKT & 177.0139 & -37.9817 & 0.5 & 3.2 & -14.1 & 1.6 & 2.0 & 5.5 & 4.3 & 1.3 & -13.0 & 0.9 & 1.7 & 3.4 & 3370 & 5160 \\
\hline WHVR & 175.4517 & -39.7301 & -10.6 & 2.9 & -1.0 & 1.8 & 3.2 & 6.1 & -11.2 & 1.2 & -1.6 & 1.3 & 3.9 & 3.1 & 4292 & 5160 \\
\hline WITH & 173.9843 & -41.5606 & -4.7 & 30.6 & -5.5 & 5.4 & -1.1 & 7.7 & -23.0 & 1.4 & -1.5 & 1.2 & 1.9 & 3.5 & 3602 & 5160 \\
\hline WMAT & 178.4087 & -37.8250 & 3.9 & 3.5 & -21.3 & 1.9 & 2.0 & 5.9 & -7.6 & 1.4 & -20.6 & 1.4 & 6.8 & 4.0 & 3727 & 5159 \\
\hline WPAW & 176.5430 & -39.8959 & -15.0 & 5.8 & -12.6 & 2.1 & -1.5 & 7.8 & -20.0 & 1.9 & -10.6 & 1.0 & -4.5 & 4.3 & 2303 & 5160 \\
\hline WPUK & 176.4406 & -40.0642 & -17.3 & 5.4 & -12.1 & 3.4 & -0.5 & 7.8 & -20.7 & 2.0 & -9.4 & 1.2 & -3.9 & 4.1 & 2302 & 5160 \\
\hline
\end{tabular}

Site name is that used by the GeoNet cGPS network

$\mathrm{Ve}$ is towards the east, $\mathrm{Vn}$ is towards the north and $\mathrm{Vu}$ is upwards

'RMS' is root mean square residual to a linear fit for the full time series used 


\section{Appendix 12 - Regional strain fields from secular velocity data (ref. 3.3)}

\begin{tabular}{|c|c|c|c|c|c|c|c|c|c|c|}
\hline \multirow[t]{2}{*}{ Region } & \multicolumn{5}{|c|}{ SSE-free } & \multicolumn{5}{|c|}{ Average SSE } \\
\hline & $\begin{array}{l}\text { Ppal strain } 1 \\
\text { extensional } \\
\text { (10E-9/yr) }\end{array}$ & $\begin{array}{l}\mathrm{Az} \\
\text { extensional }\end{array}$ & $\begin{array}{l}\text { Ppal strain } 2 \\
(10 \mathrm{E}-9 / \mathrm{yr})\end{array}$ & $A z 2$ & $\begin{array}{l}\text { Rotation } \\
\text { rate } \\
(\% / \mathrm{Ma})\end{array}$ & $\begin{array}{l}\text { Ppal strain } 1 \\
\text { extensional } \\
\text { (10E-9/yr) }\end{array}$ & $\begin{array}{l}\mathrm{Az} \\
\text { extensional }\end{array}$ & $\begin{array}{l}\text { Ppal strain } 2 \\
(10 \mathrm{E}-9 / \mathrm{yr})\end{array}$ & $A z 2$ & $\begin{array}{l}\text { Rotation } \\
\text { rate } \\
(\% / \mathrm{Ma})\end{array}$ \\
\hline NE coast 9 sites & $78(1)$ & $071(2)$ & $-65(8)$ & $161(2)$ & $7.6(0.1)$ & $71(1)$ & $168(4)$ & $-11(8)$ & $078(4)$ & $-2.8(0.1)$ \\
\hline Mahi-Gisborne & $49(20)$ & $165(5)$ & $-97(24)$ & $075(5)$ & n.r. & $90(23)$ & $075(9)$ & $-4(20)$ & $165(9)$ & n.r. \\
\hline Alt. NE coast 14 sites & $31(4)$ & $069(6)$ & $-15(8)$ & $159(6)$ & $4.9(0.1)$ & $41(4)$ & $159(6)$ & $-8(9)$ & $069(6)$ & $-1.9(0.1)$ \\
\hline Southern Hawkes Bay & $53(6)$ & $092(5)$ & $+4(4)$ & $002(5)$ & $4.3(0.1)$ & $24(6)$ & $114(8)$ & $-8(5)$ & $024(8)$ & $0.6(0.1)$ \\
\hline Wellington/Wairarapa & $12(2)$ & $178(1)$ & $-120(1)$ & $088(1)$ & $3.4(0.1)$ & $1(2)$ & $173(4)$ & $-21(1)$ & $083(4)$ & $0.1(0.1)$ \\
\hline Manawatu & $37(3)$ & $131(2)$ & $-26(2)$ & $041(2)$ & $4.1(0.1)$ & $8(3)$ & $052(10)$ & $-3(2)$ & $142(10)$ & $-0.7(0.1)$ \\
\hline Ruapehu & $32(10)$ & $131(14)$ & $-51(38)$ & $041(14)$ & $3.2(0.1)$ & $15(10)$ & $029(56)$ & $-17(39)$ & $119(56)$ & $-0.9(0.1)$ \\
\hline TVZ & $39(9)$ & $141(7)$ & $-18(10)$ & $051(7)$ & $2.3(0.1)$ & $-1(10)$ & $141(54)$ & $-12(10)$ & $051(54)$ & $0.0(0.1)$ \\
\hline All sites & $12(3)$ & $148(13)$ & $+2(2)$ & $058(13)$ & $3.8(0.3)$ & $17(3)$ & $104(5)$ & $-1(2)$ & $014(5)$ & $0.0(0.1)$ \\
\hline
\end{tabular}

+ve strain rates are extensional and +ve rotation rates are clockwise. SSE-free poles are located within the region. Avg. SSE poles are distant and external to the region (except for Ruapehu). Values in parentheses are $95 \%$ confidence limits for the specific site combinations shown below. n.r. - not considered reliable.

On the following pages, plots for each region show respectively:

\begin{tabular}{|l|l|}
\hline $\begin{array}{l}\text { Secular velocity components remaining after deduction of reference site velocities. } \\
\text { Actual site secular velocities shown for the reference site only. Red = overall } \\
\text { average, blue = SSE-free, black = SSE only. }\end{array}$ & $\begin{array}{l}\text { Normalised strain rate ellipse for SSE-free velocities* } \\
\text { * azimuths are shown correctly in ellipses but in some cases azimuth values are incorrectly assigned to } \\
\text { the principal strain axes in the annotation }\end{array}$ \\
\hline $\begin{array}{l}\text { Normalised strain rate ellipse for average SSE velocities* } \\
\text { * azimuths are shown correctly in ellipses but in some cases azimuth values are incorrectly assigned to } \\
\text { the principal strain axes in the annotation }\end{array}$ & $\begin{array}{l}\text { Asymmetric components of residuals after removal of symmetric (strain) } \\
\text { components, together with best estimate rotation pole for SSE-free velocities. Cyan } \\
\text { lines indicate actual residuals and black lines indicate least squares modelled } \\
\text { rotation components. }\end{array}$ \\
\hline $\begin{array}{l}\text { Asymmetric component of residuals after removal of symmetric (strain) component } \\
\text { for average SSE velocities. Rotation pole is usually outside the region. }\end{array}$ & SSE-free pole location estimates from 500 Monte Carlo simulations \\
\hline
\end{tabular}


Appendices

173

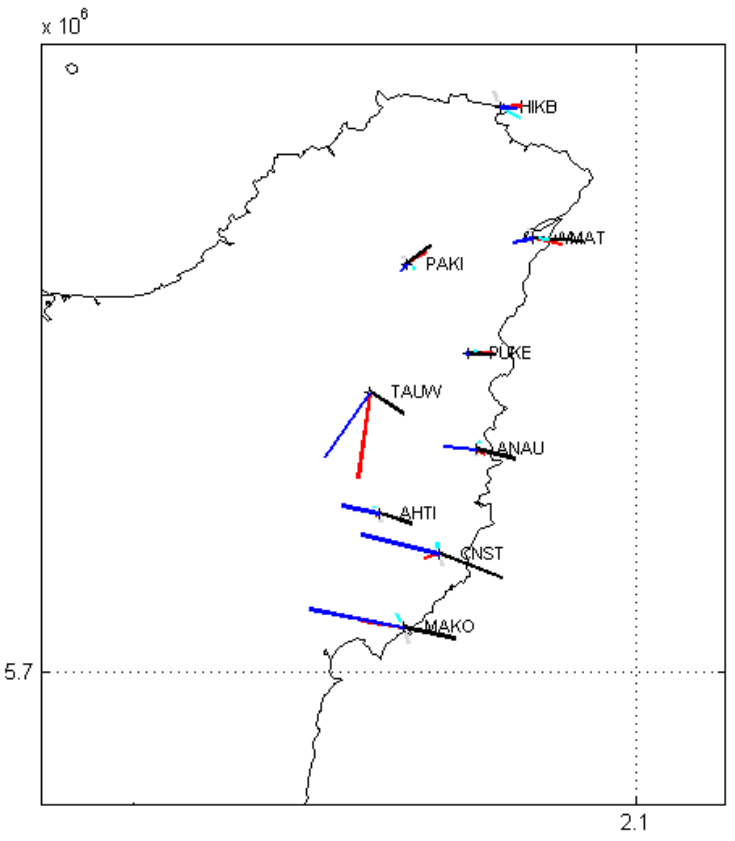

Strain ellipse-SSE vel $\times 2.9 e+06$
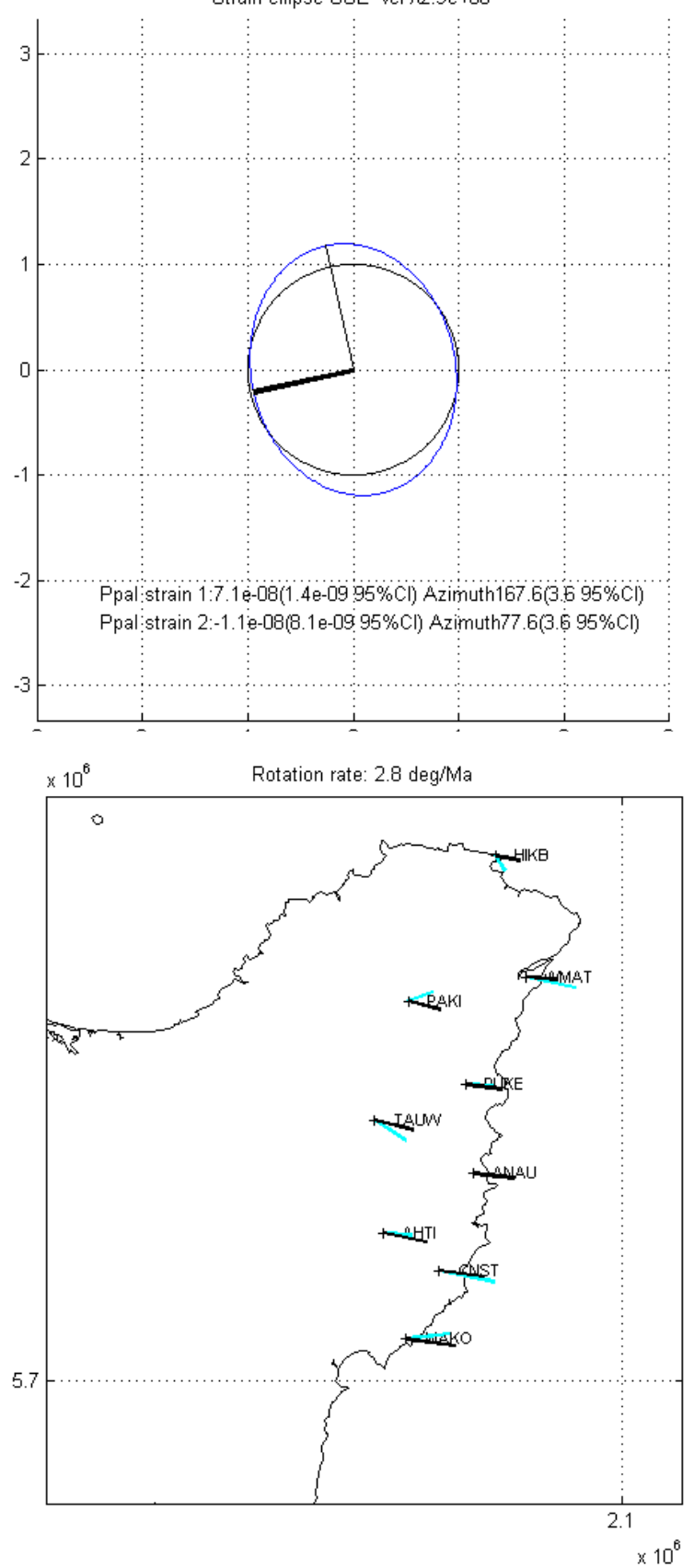

$\underline{\text { Northeast coast }-9 \text { sites }}$
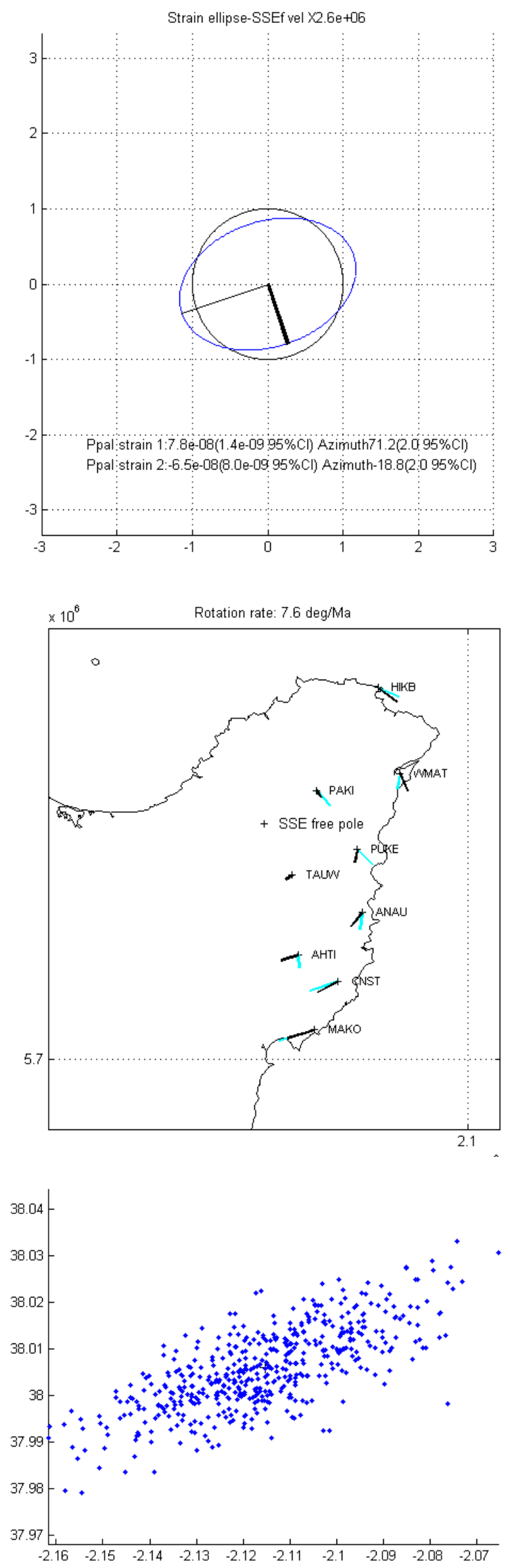

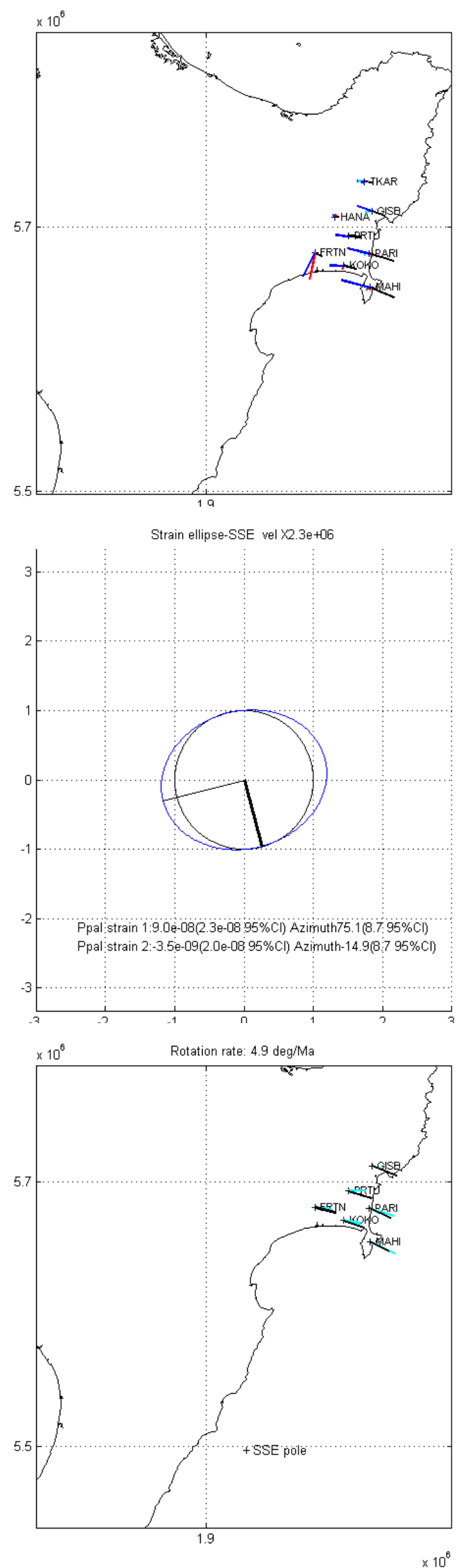

Mahi - Gisborne
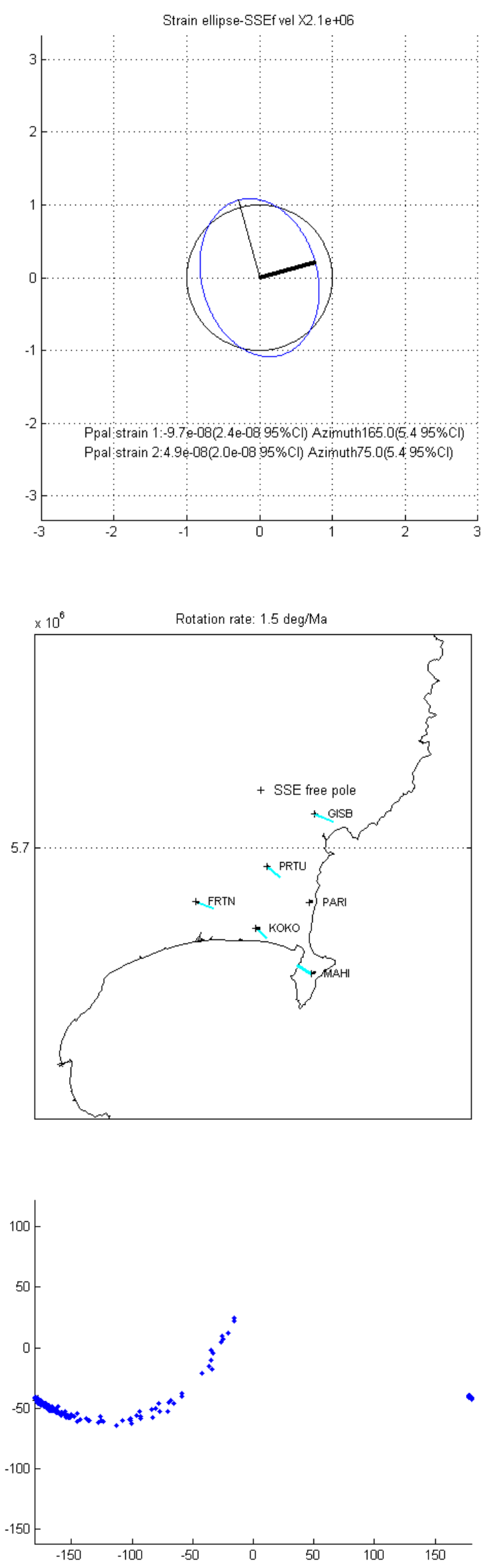
ICK
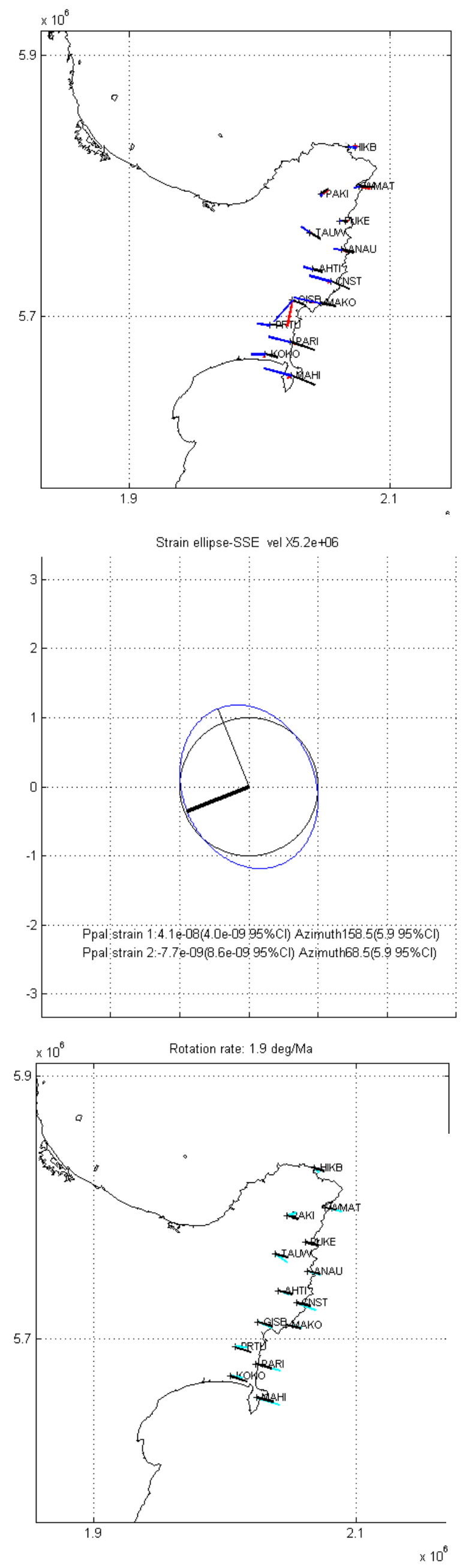

\section{$\underline{\text { Alternative - NE coast } 14 \text { sites }}$}
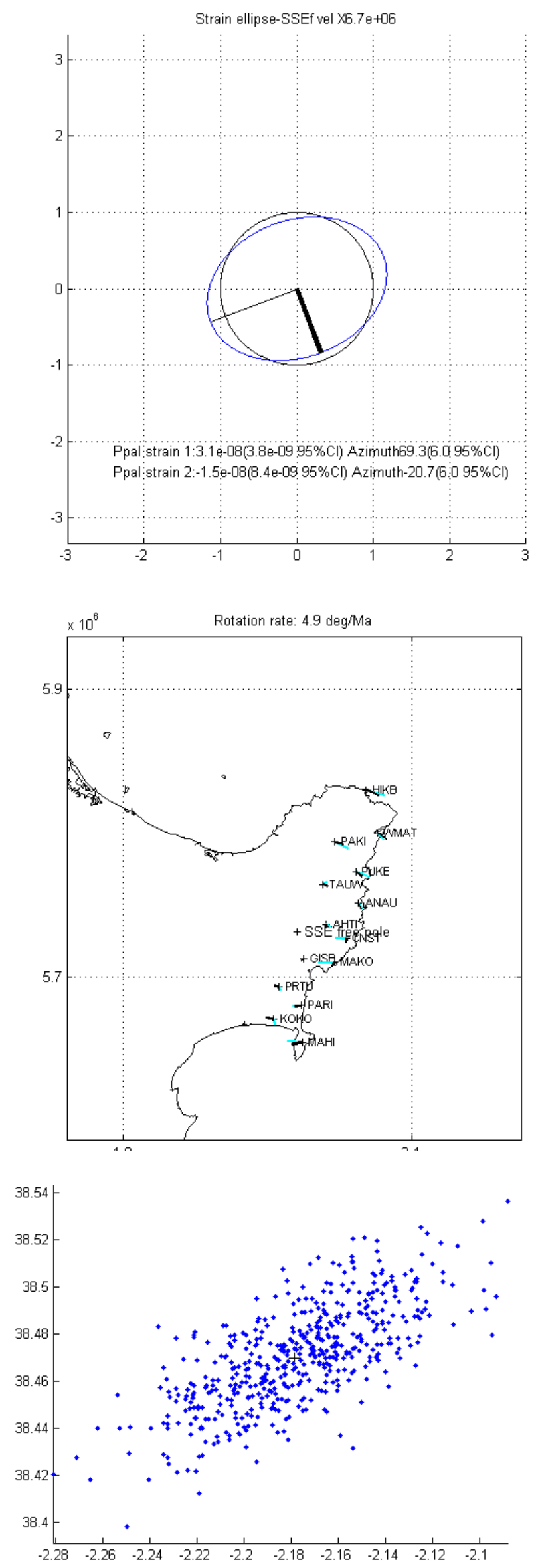

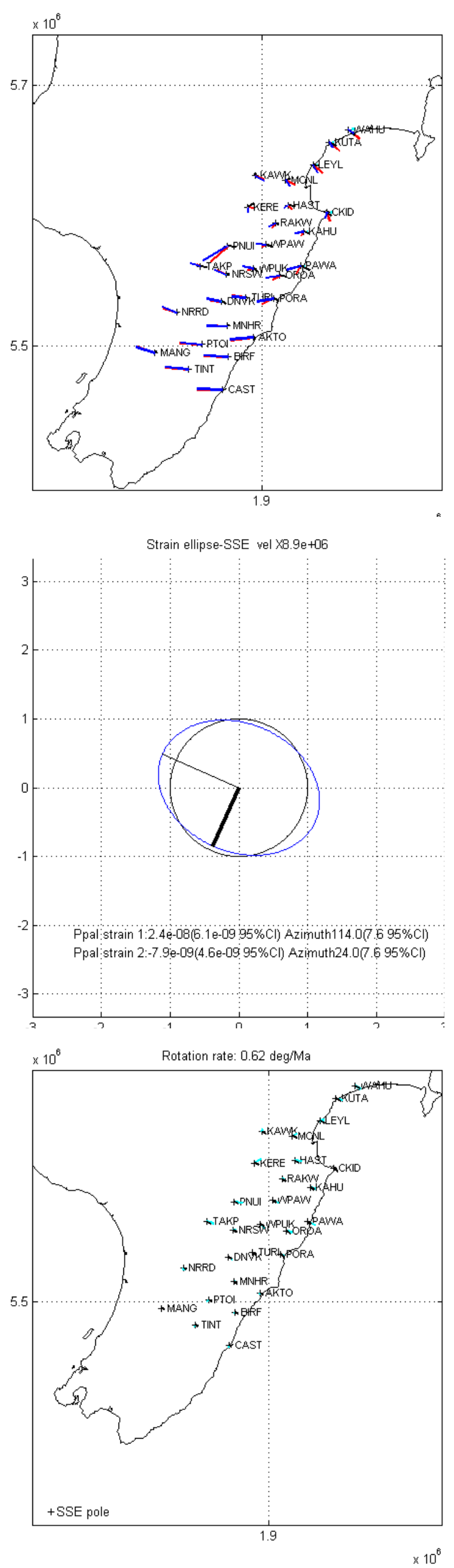

\section{$\underline{\text { Southern Hawkes Bay }}$}
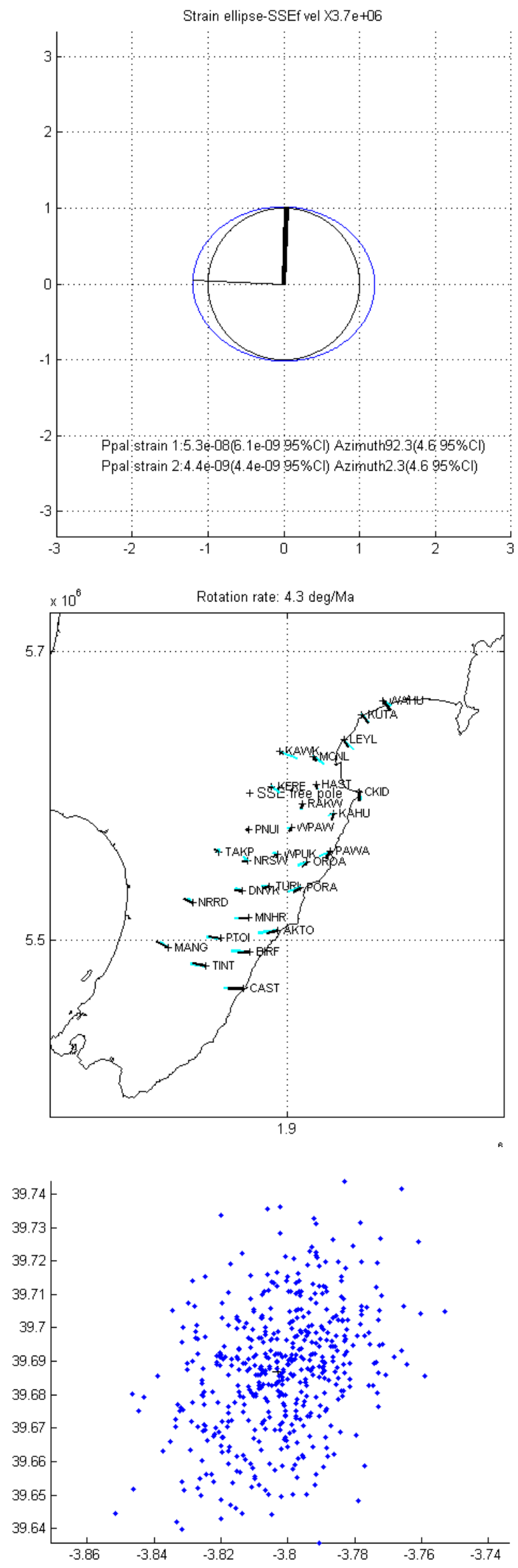

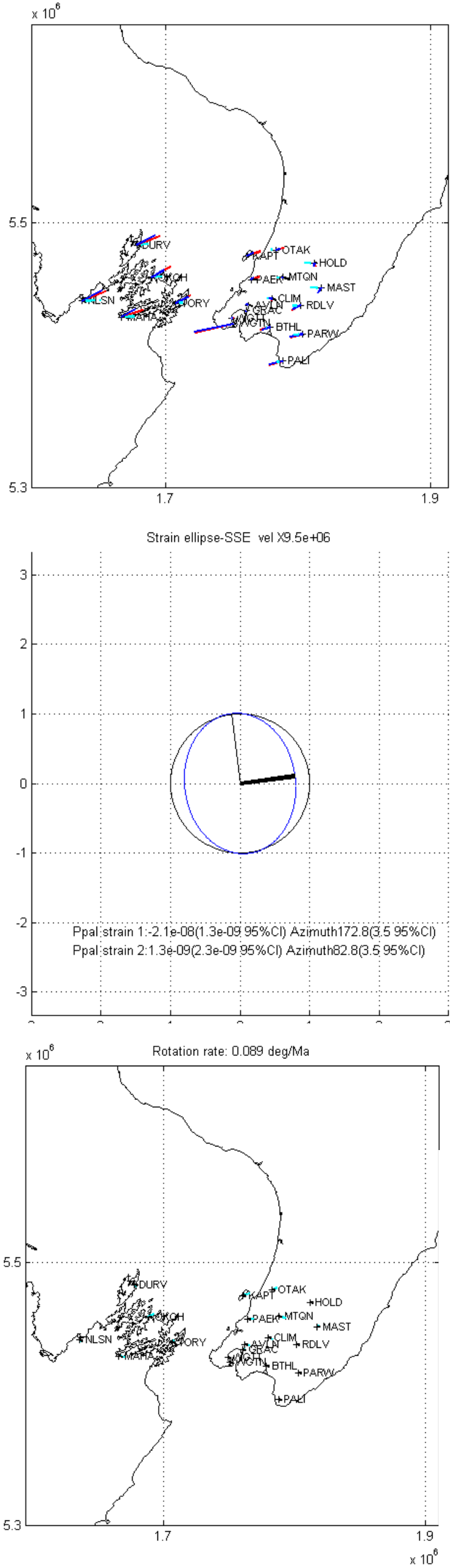

\section{Wellington \& south Wairarapa}
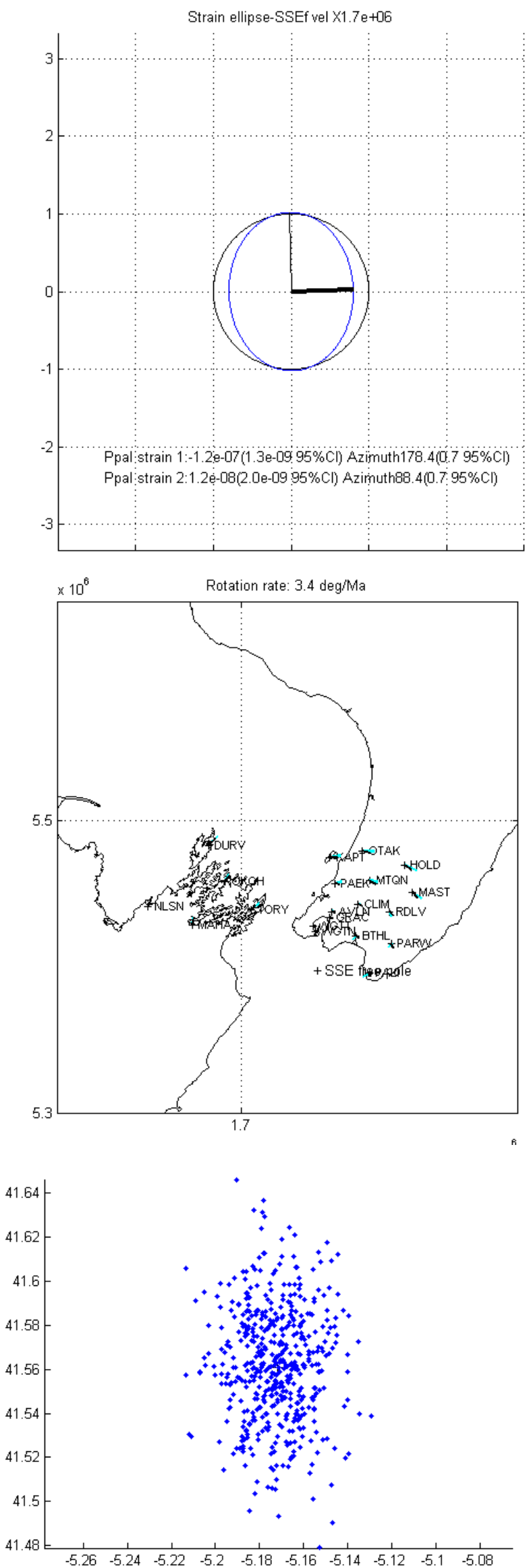


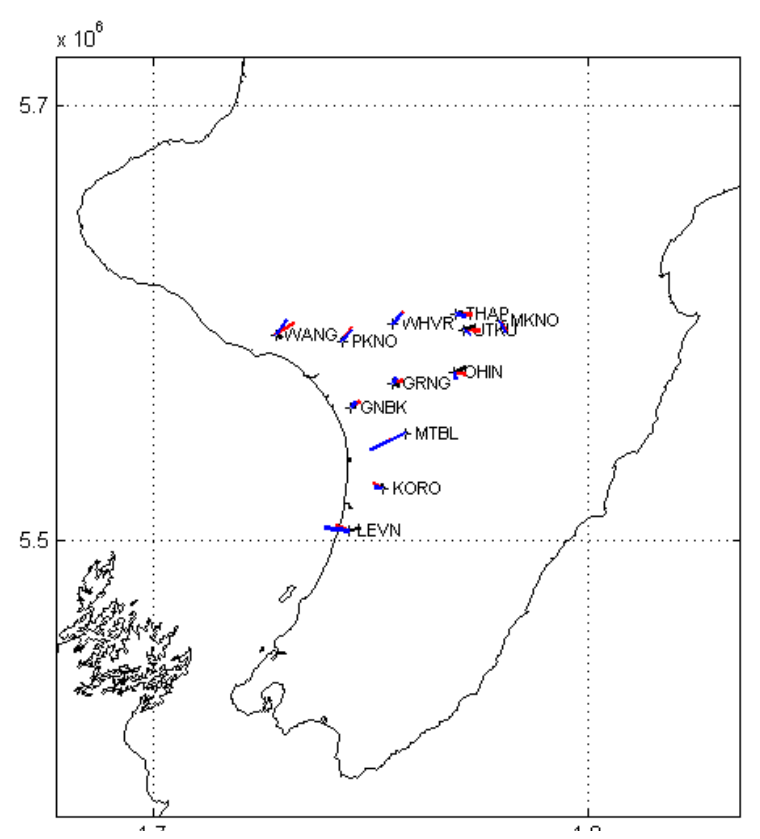

Strain ellipse-SSE vel $\times 3 e+07$
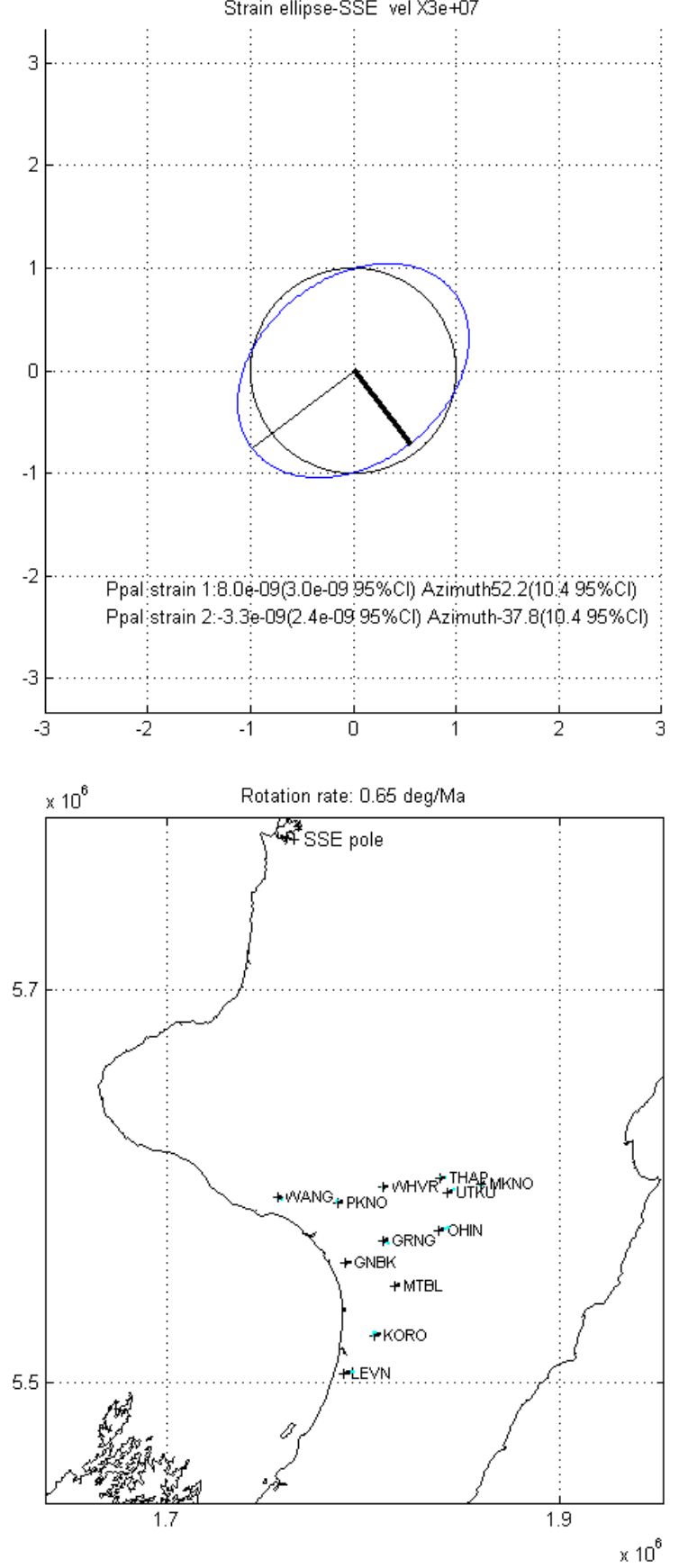

\section{Manawatu}
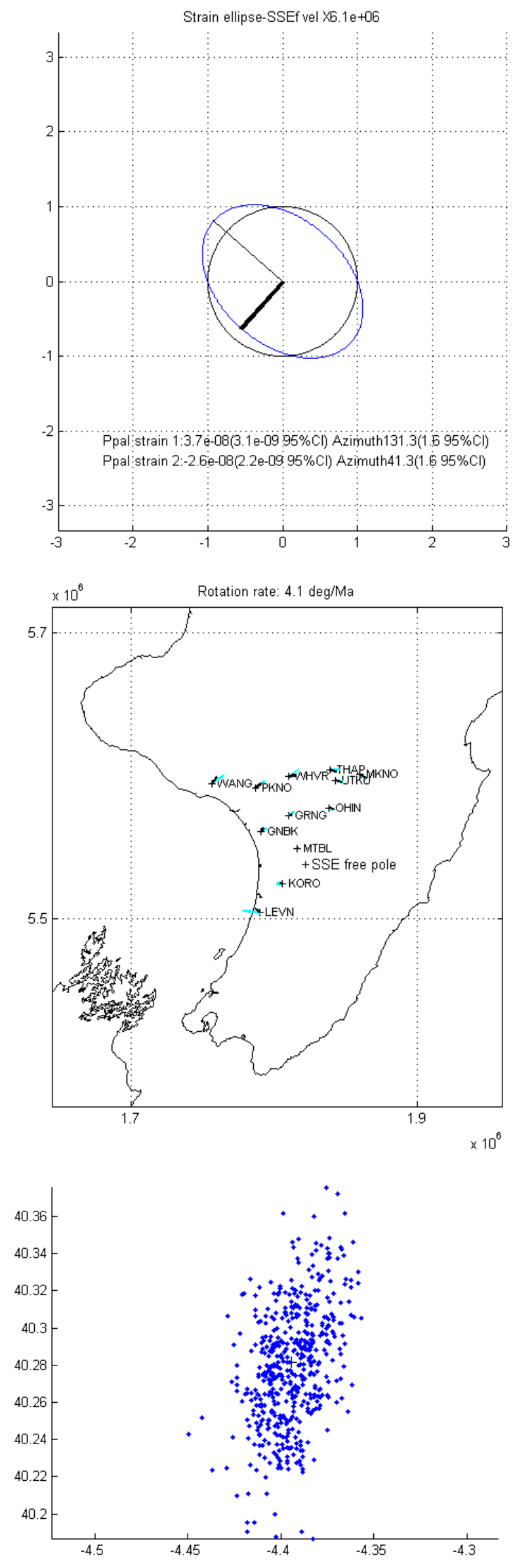


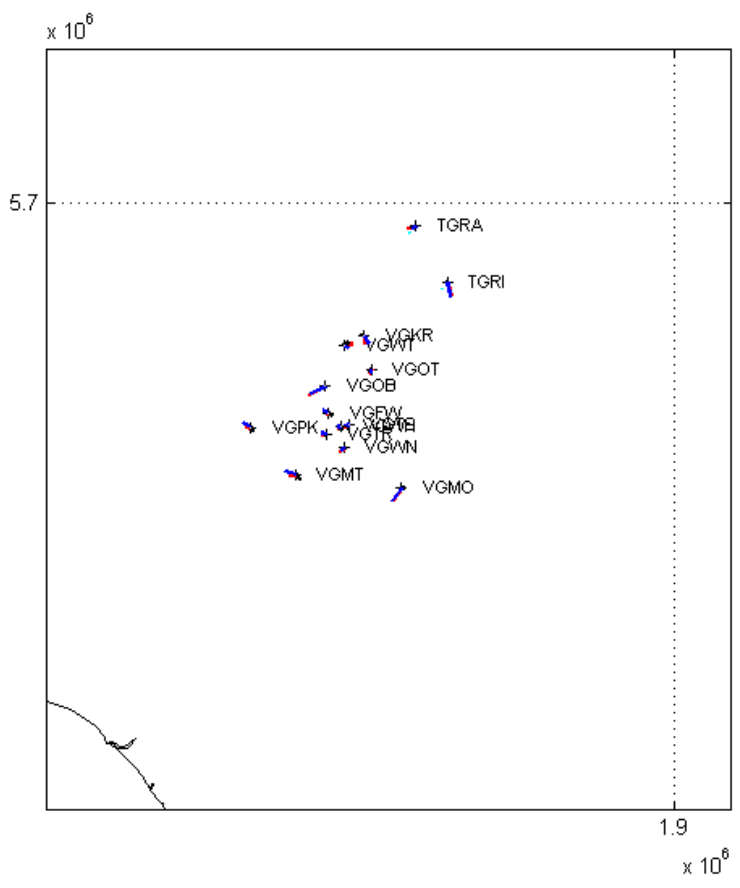

Strain ellipse-SSE vel $\times 1.2 \mathrm{e}+07$
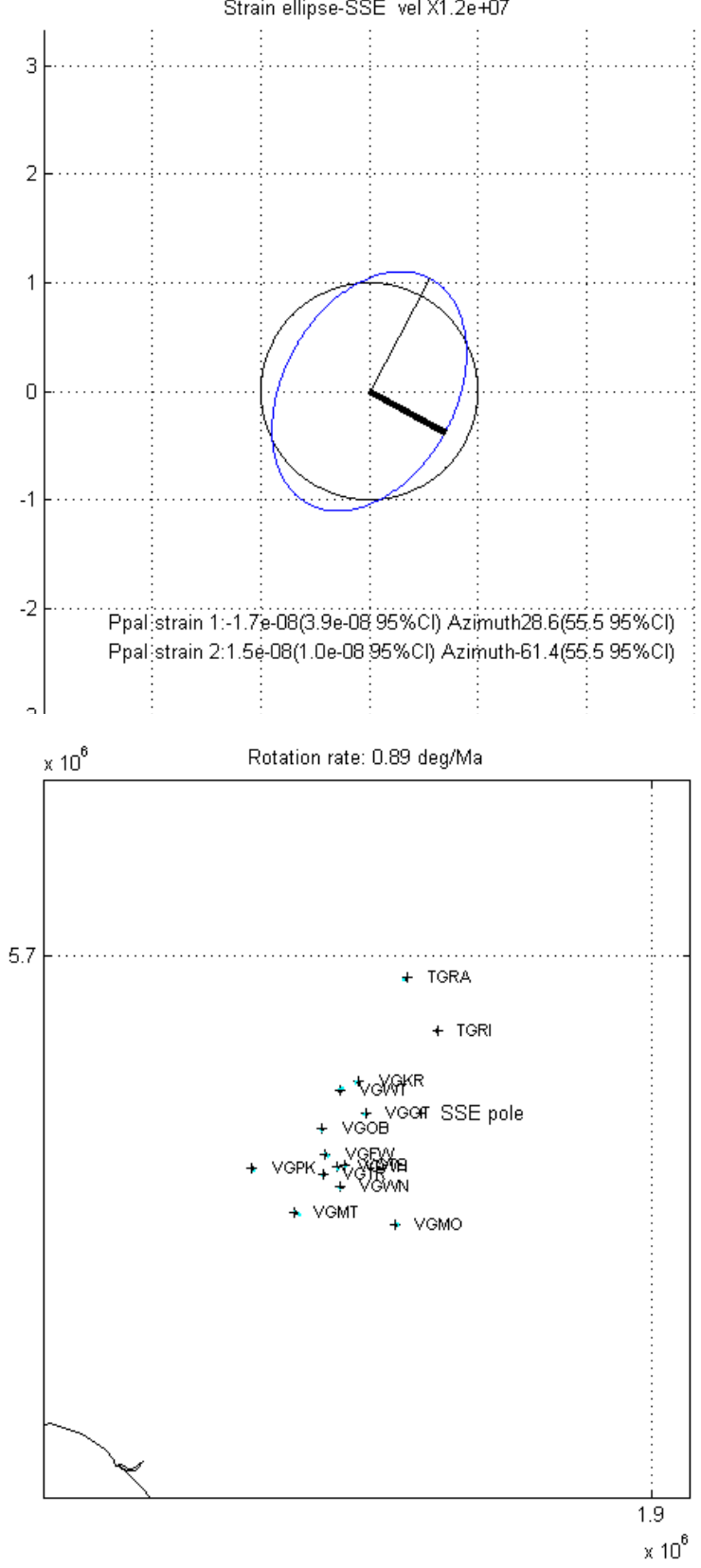

\section{Ruapehu}
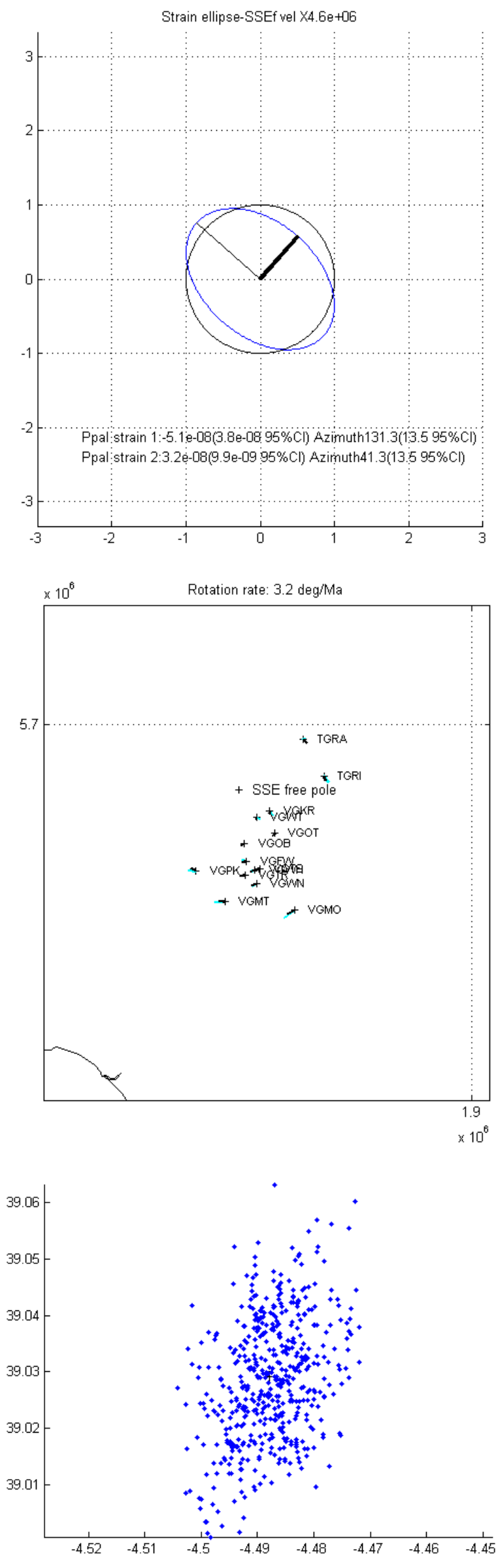

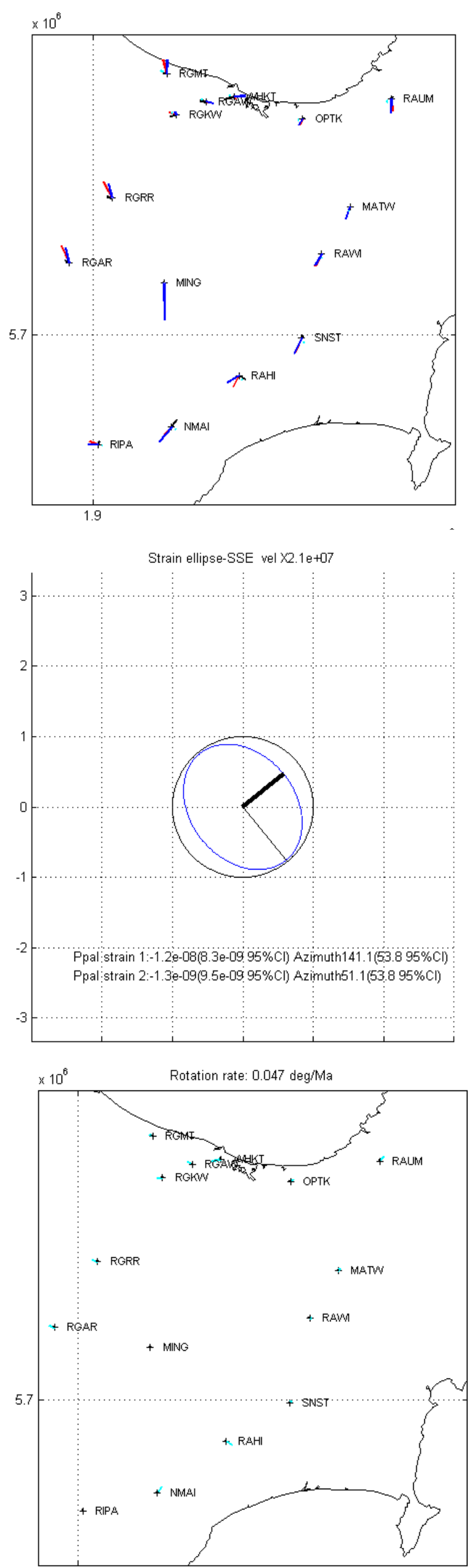

1.9

\section{$\underline{\text { Taupo Volcanic Zone }}$}
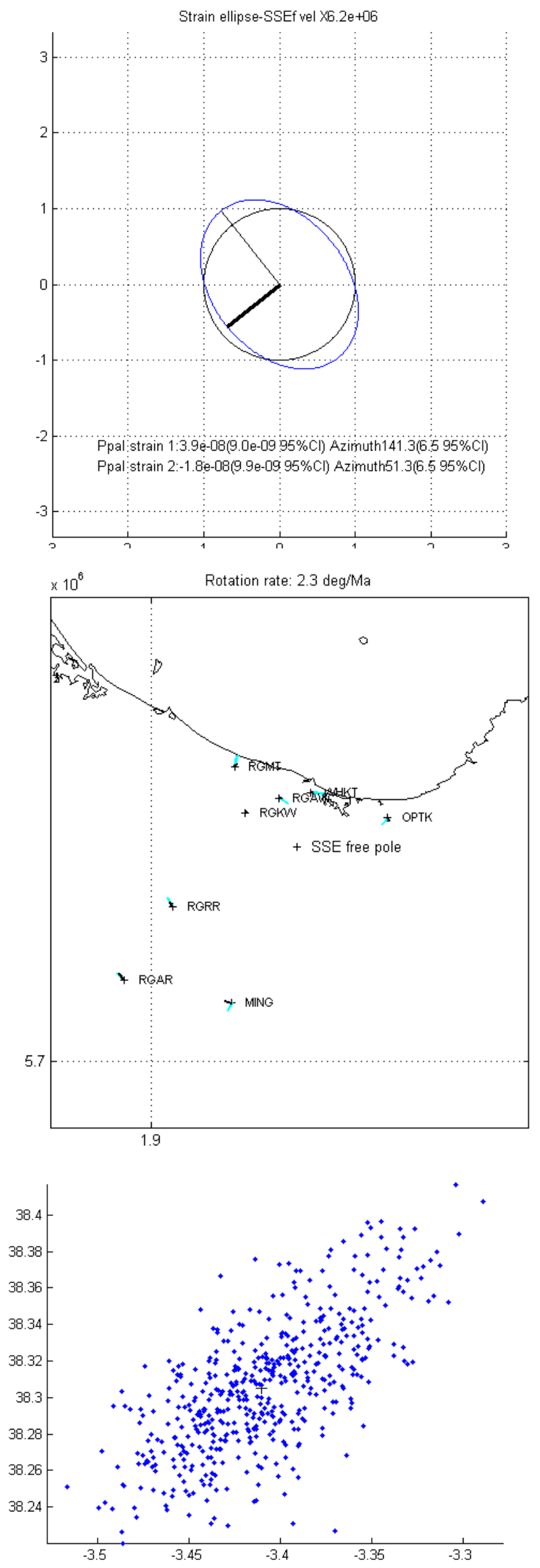


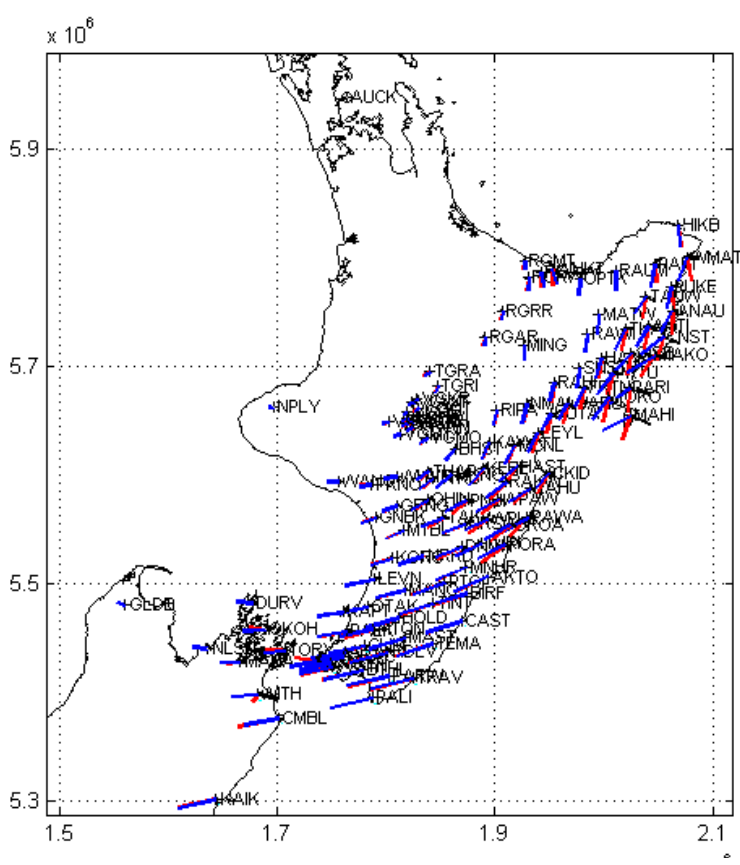

Strain ellipse-SSE vel $\times 1.2 e+07$
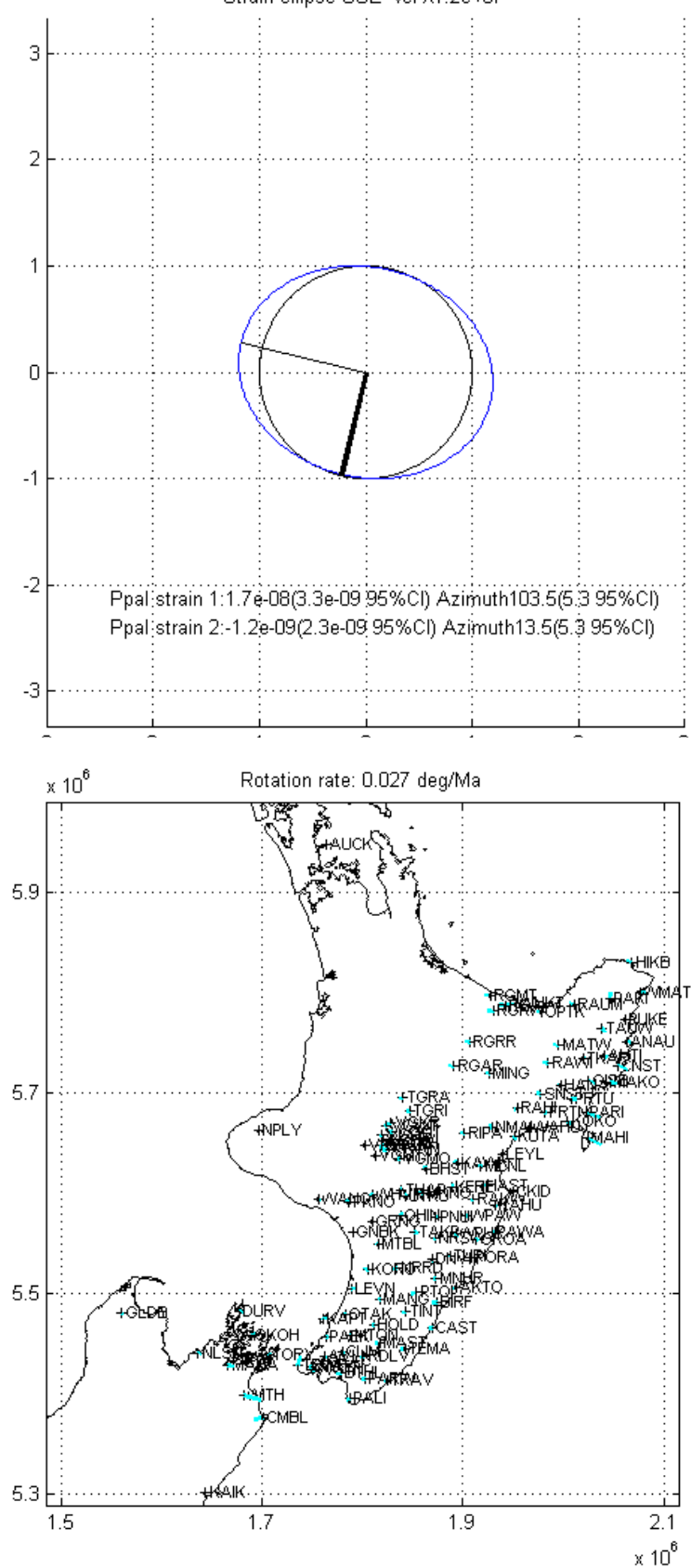

\section{$\underline{\text { All sites }}$}
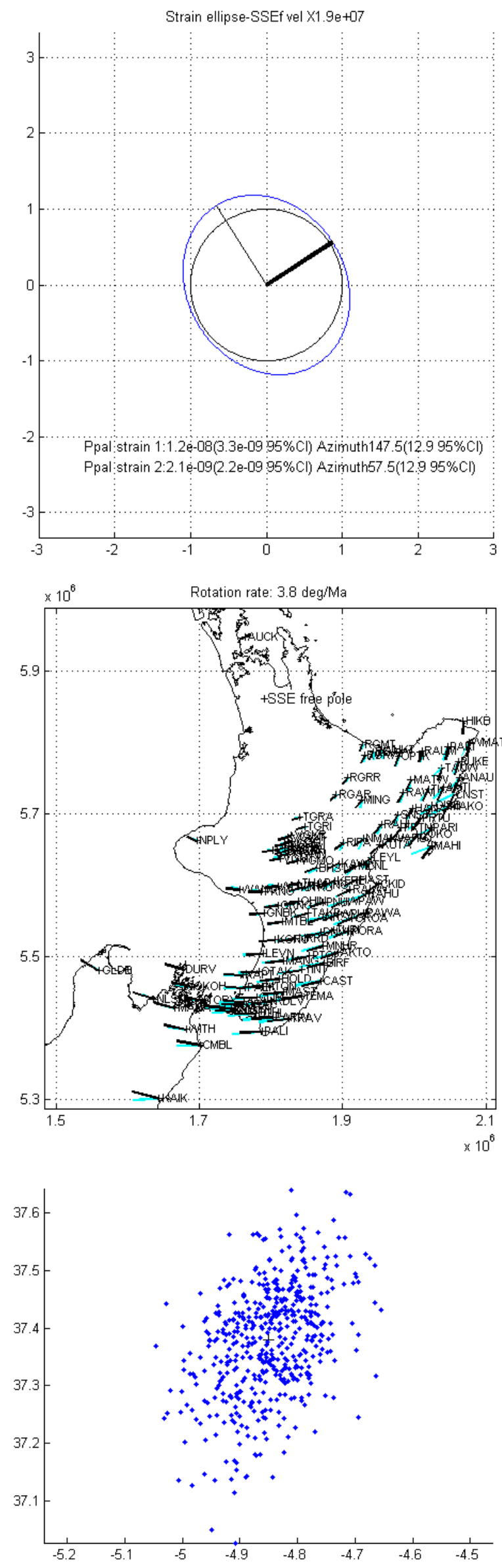


\section{Appendix 13 - Parametric study of the effects of layering}

(ref. 4.5) The following method was used for this study. For each parameter combination, input files were generated for EDGRN and EDCMP. In the case of the Okada solution, depth-weighted 'average' velocities and densities were converted to Lamé parameters. In each case, the code was run in both Okada and layered modes, and the full surface displacement data from each solution (across (Y) and along (X) strike above the centre of the length of the fault) were plotted, and the differences calculated across the full profile. For the purpose of comparing the parametric effects, the maximum difference for each parametric combination was calculated as a percentage of the maximum Okada displacement. An example of an individual set of comparisons (from changes in rake) is shown in Fig. A13a.

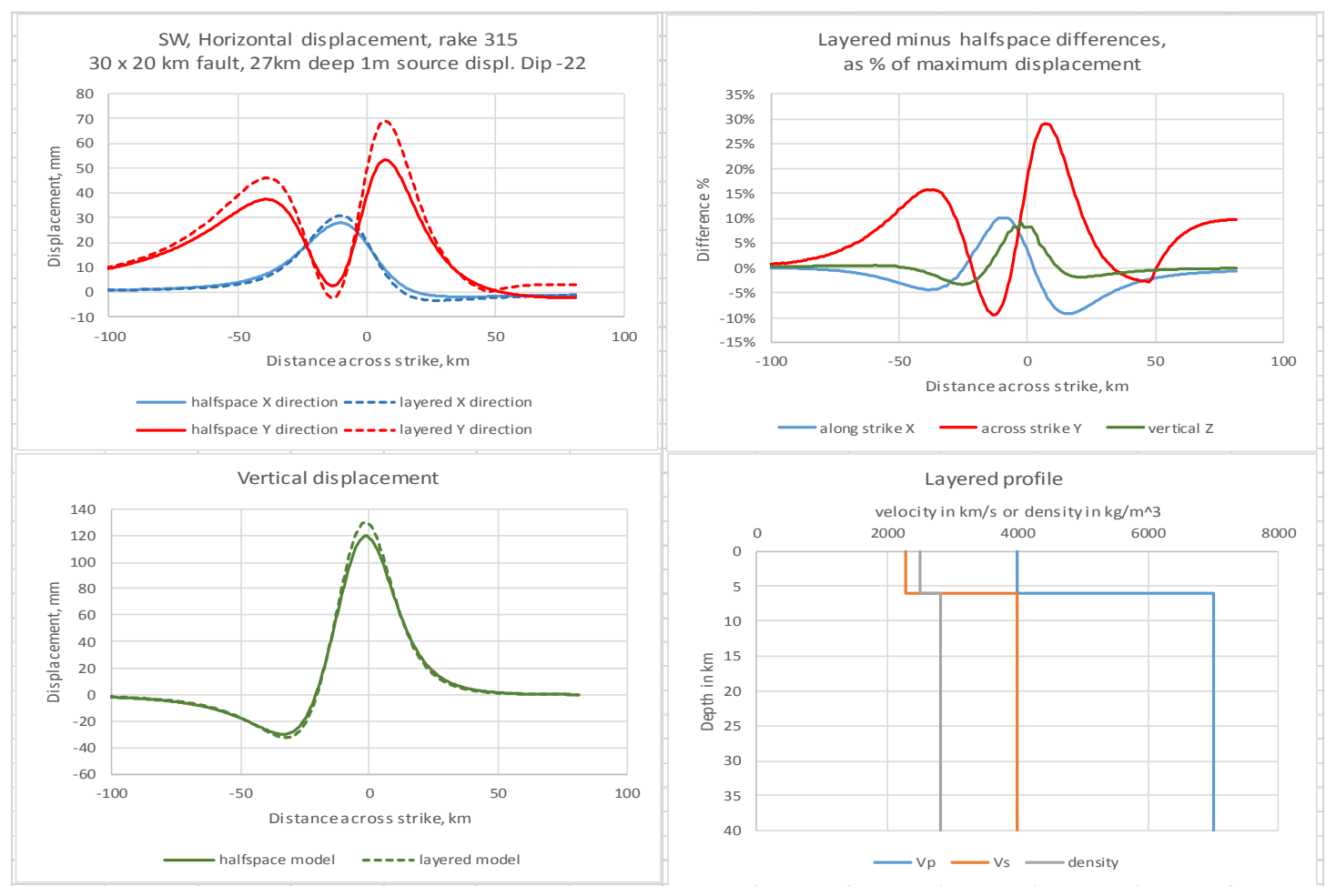

Figure A13a: Clockwise from top left, the plots show: surface displacement in $\mathrm{X}$ and $\mathrm{Y}$ directions along and across the strike respectively, above the centre of the length of the fault; percentage change in surface displacement as a proportion of the maximum in the Okada model; the profile of $\mathrm{V}_{\mathrm{P}}, \mathrm{V}_{\mathrm{S}}$, and density with depth used in the layered model; and the surface displacements in the upwards direction across the strike of the fault.

Analyses were conducted to simulate SSEs in the northeast (NE) and southwest (SW) of the North Island. 


\section{Part 1: Shallow thrust fault (NE of South Island)}

A buried fault, $30 \mathrm{~km}$ long (along strike) and $20 \mathrm{~km}$ wide (down dip), was modeled using the EDGRN/EDCMP layer code and Okada's homogenous half-space code, and the results compared. In the plots below, the results are simplified by plotting only the maximum displacements at the surface in the horizontal and vertical directions. In all cases the source movement is taken as $1 \mathrm{~m}$ perpendicular to the strike. The differences (bold lines, right hand $\mathrm{Y}$ scale) are expressed as percentages of the maximum displacement produced by the Okada model. In all cases the lower half-space has properties of $\mathrm{Vp}=7000 \mathrm{~m} / \mathrm{s}, \mathrm{Vs}=4000 \mathrm{~m} / \mathrm{s}$ and density $=2800 \mathrm{~kg} / \mathrm{m}^{3}$. The equivalent half-space model used in the Okada code is calculated, for a total depth of $40 \mathrm{~km}$, in each case using the depth-weighted modulus-equivalent (i.e. using velocity ${ }^{2}$ values), although how this is calculated has negligible effect on the comparison. Except where the elastic properties of the upper layer are varied, they otherwise take the values of $\mathrm{Vp}$ $=4000 \mathrm{~m} / \mathrm{s}, \mathrm{Vs}=2286 \mathrm{~m} / \mathrm{s}$, and density $=2500 \mathrm{~kg} / \mathrm{m}^{3}$. In all simulations, Poisson's ratio $=0.26$.

The parameters explored are:

- Change in dip angle from $-10^{\circ}$ to $-18^{\circ}$, depth to fault top $=18 \mathrm{~km}$, depth of upper layer $=6 \mathrm{~km}$, elastic properties held fixed

- Change in fault depth from 10 to $26 \mathrm{~km}$, dip $=-12^{\circ}$, depth of upper layer $=6 \mathrm{~km}$, elastic properties held fixed

- Change in depth of upper layer from 2 to $10 \mathrm{~km}$, fault depth $=18 \mathrm{~km}$, dip $=-12^{\circ}$, elastic properties held fixed

- Change in elastic properties of upper layer, by varying Vp from 2000 to 6000 $\mathrm{m} / \mathrm{s}$, Vs from 1143 to $3429 \mathrm{~m} / \mathrm{s}$ (i.e. keeping ratio constant), and density from 2100 to $2700 \mathrm{~kg} / \mathrm{m}^{3}$ (giving E values ranging from $5.5 \mathrm{E}+09$ to $6.3 \mathrm{E}+10 \mathrm{~Pa}$ ). Upper layer depth held constant at $6 \mathrm{~km}$, fault depth $=18 \mathrm{~km}$, and dip $=12^{\circ}$.

The first three models are illustrated in the simulated cross section, across strike, shown in Fig. A13b. 


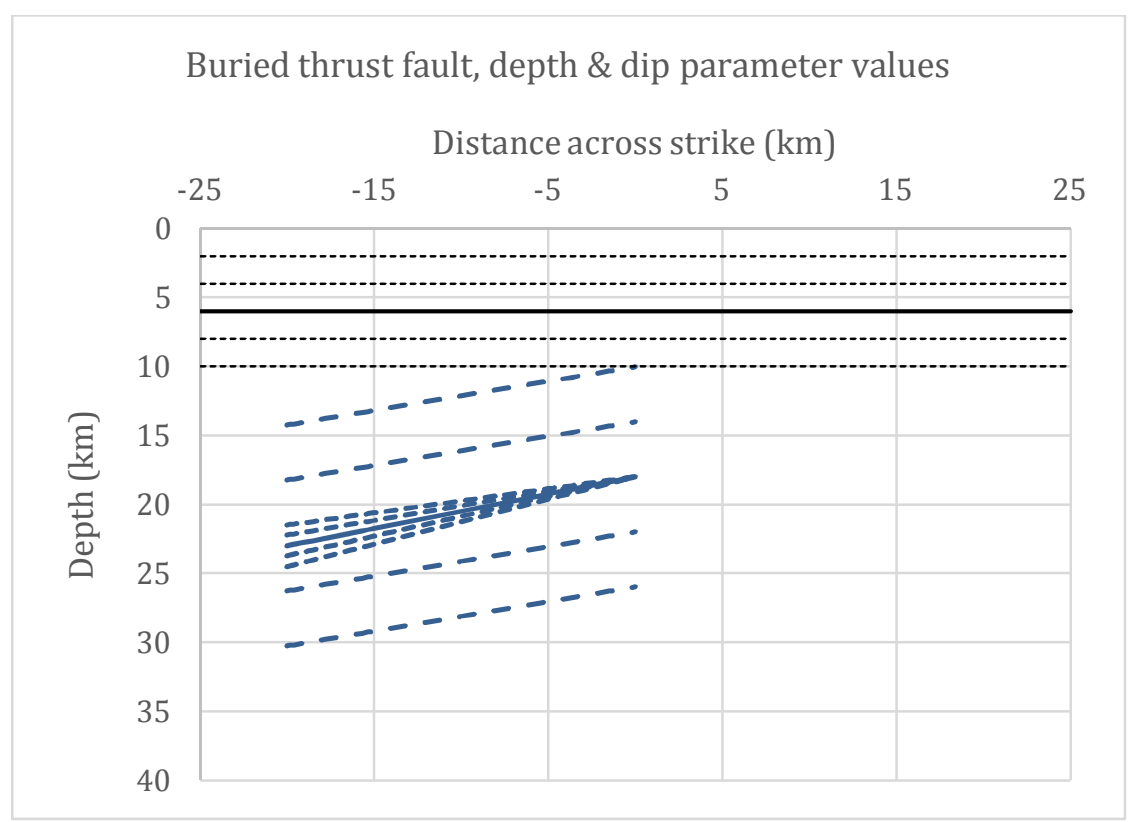

Figure A13b: Cross-section along strike illustrating the geometries examined in the shallow thrust fault simulation. Bold continuous lines indicate default values when other parameters are varied. Dashed lines indicate the range of dips and depths explored in the analyses. The horizontal black lines indicate the boundary between the upper layer and the half-space.

Fig. A13c illustrates variations in surface displacements and relative differences for the two models over the range of shallow thrust fault models indicated above. These plots indicate that:

- Variations in dip angle, over the range $10^{\circ}$ to $18^{\circ}$ have little significant effect on comparisons between surface displacements between the Okada and layered models. The differences remain constant in both the $\mathrm{Y}$ and $\mathrm{Z}$ components.

- Variations in the depth of the fault, over the range 12 to $26 \mathrm{~km}$ have a small effect $(\sim 10 \%)$ only on the comparison on the vertical displacements (Uz). At less than $11 \mathrm{~km}$ depth, the projection of the fault increasingly intersects the boundary of the upper layer, causing significantly increasing displacements.

- Variations in the upper layer thickness from 2 to $10 \mathrm{~km}$ has a small effect (from $\sim 3$ to $\sim 8 \%$ ) on the comparison of vertical displacements but a much more significant effect on horizontal displacements ( 17 to $\sim 33 \%)$.

- Variations in elasticity ratio between the upper layer and the half-space appear to have a significant effect on the comparison in both the vertical and horizontal directions. As the velocity in the upper layer decreases from $6 \mathrm{~km} / \mathrm{s}$ to $2 \mathrm{~km} / \mathrm{s}$, the difference in vertical displacements between the 2 models increases from $\sim 2 \%$ to $13 \%$, and that in the $\mathrm{Y}$ direction increases from $\sim 8 \%$ to $\sim 43 \%$. 

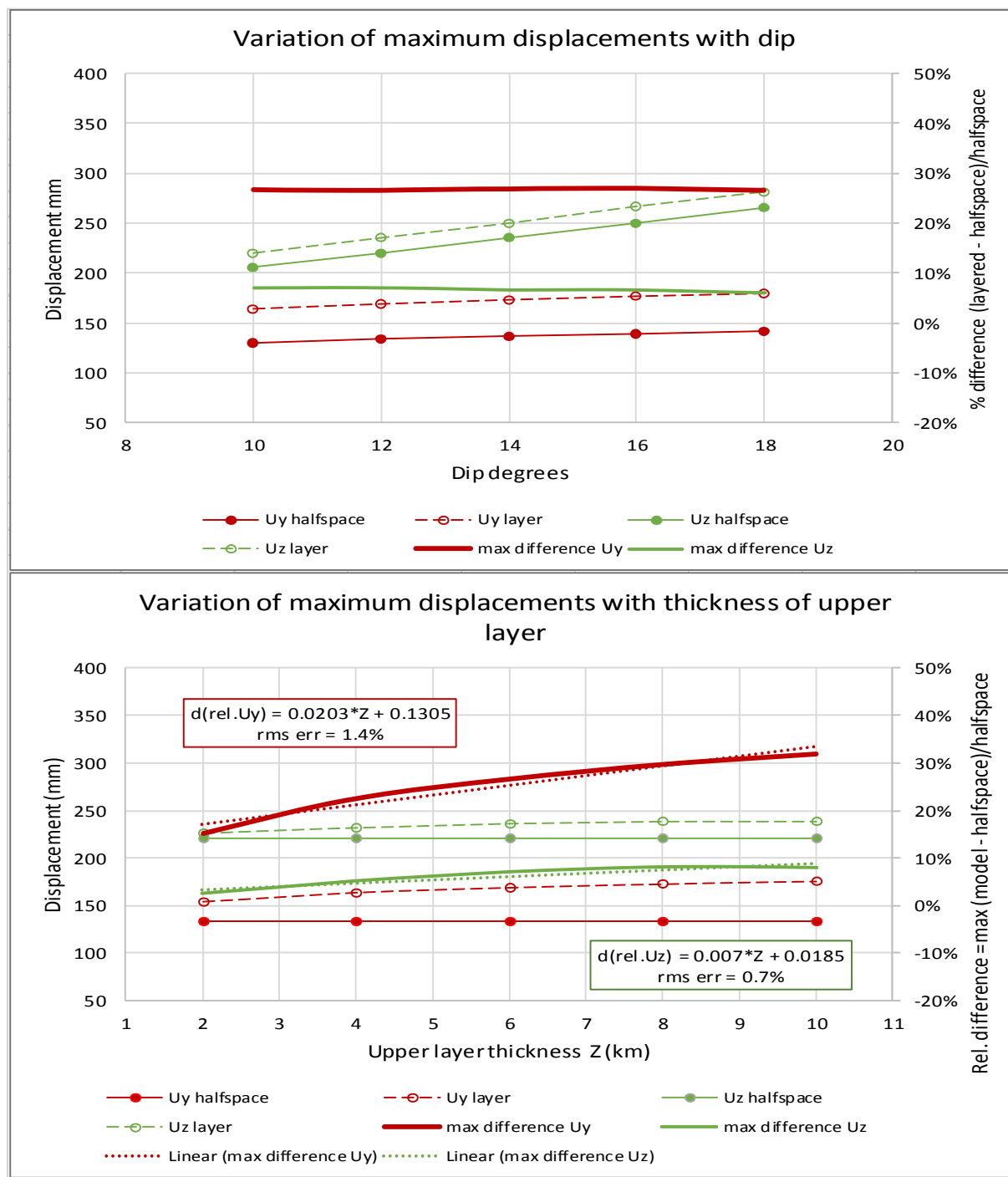

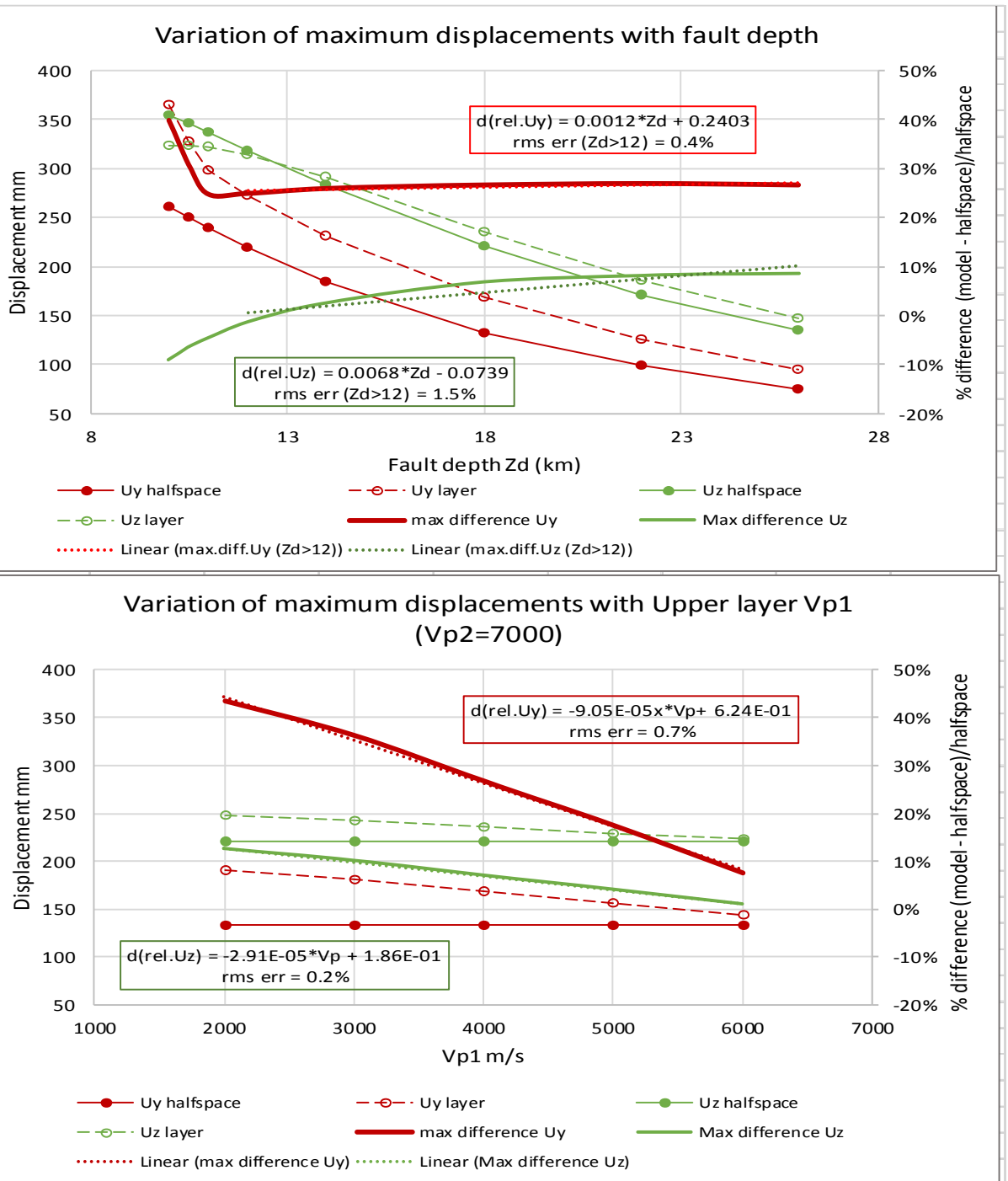


blank 
Figure A13c: Variation of surface displacements for shallow thrust fault with differing values of (in clockwise order from top left): dip (from $10^{\circ}$ to $18^{\circ}$ ); fault depth (from 10 to $26 \mathrm{~km}$ ); thickness of the upper layer (from 2 to $10 \mathrm{~km}$ ); and upper layer seismic velocity (from 2 to $6 \mathrm{~km} / \mathrm{s}$, corresponding to E ratios of 0.06, 0.15, 0.28, 0.46 and 0.71 when compared with plots in Cattin et al. [1999]). Solid lines with markers indicate maximum displacements from the Okada model, dashed lines indicate displacements from the layered model (both on the left scale), bold lines with no markers indicate percentage differences between the models (right scale), and dotted lines indicate linear regressions on the differences. Linear regression models with rms errors from linearity are shown in boxes, where relevant.

\section{Part 2: Deep strike-slip fault (SW of North Island)}

In this case an additional variant is the rake angle, which was varied from $270^{\circ}$ (pure thrust) to $360^{\circ}$ (pure strike-slip). In other respects similar conditions hold as above, except that dip is varied from $-18^{\circ}$ to $-26^{\circ}$, fault depth is varied from 17 to $50 \mathrm{~km}$, and upper layer thickness is varied from 3 to $15 \mathrm{~km}$. Default conditions are $0^{\circ}$ rake, $-22^{\circ}$ dip, $27 \mathrm{~km}$ fault depth, and upper layer thickness $6 \mathrm{~km}$. In this set of cases, the Green's function calculations are carried down to $70 \mathrm{~km}$ depth.

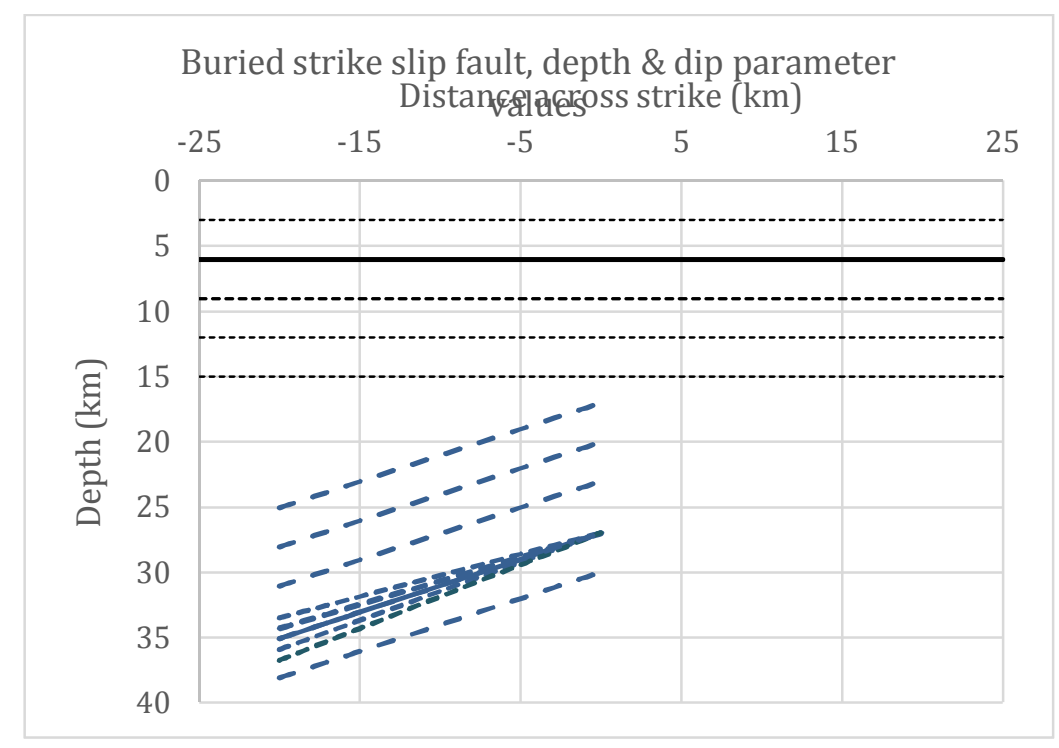

Figure A13d: Cross-section along strike illustrating the geometries examined in the deep strike-slip fault simulation. Bold continuous lines indicate default values when other parameters are varied. Dashed lines indicate the range of dips and depths explored in the analyses. The horizontal black lines indicate the boundary between the upper layer and the half-space.

Fig. A13e illustrates variations in surface displacements and relative differences for the two models over the range of deep strike-slip fault models indicated above. 
blank 


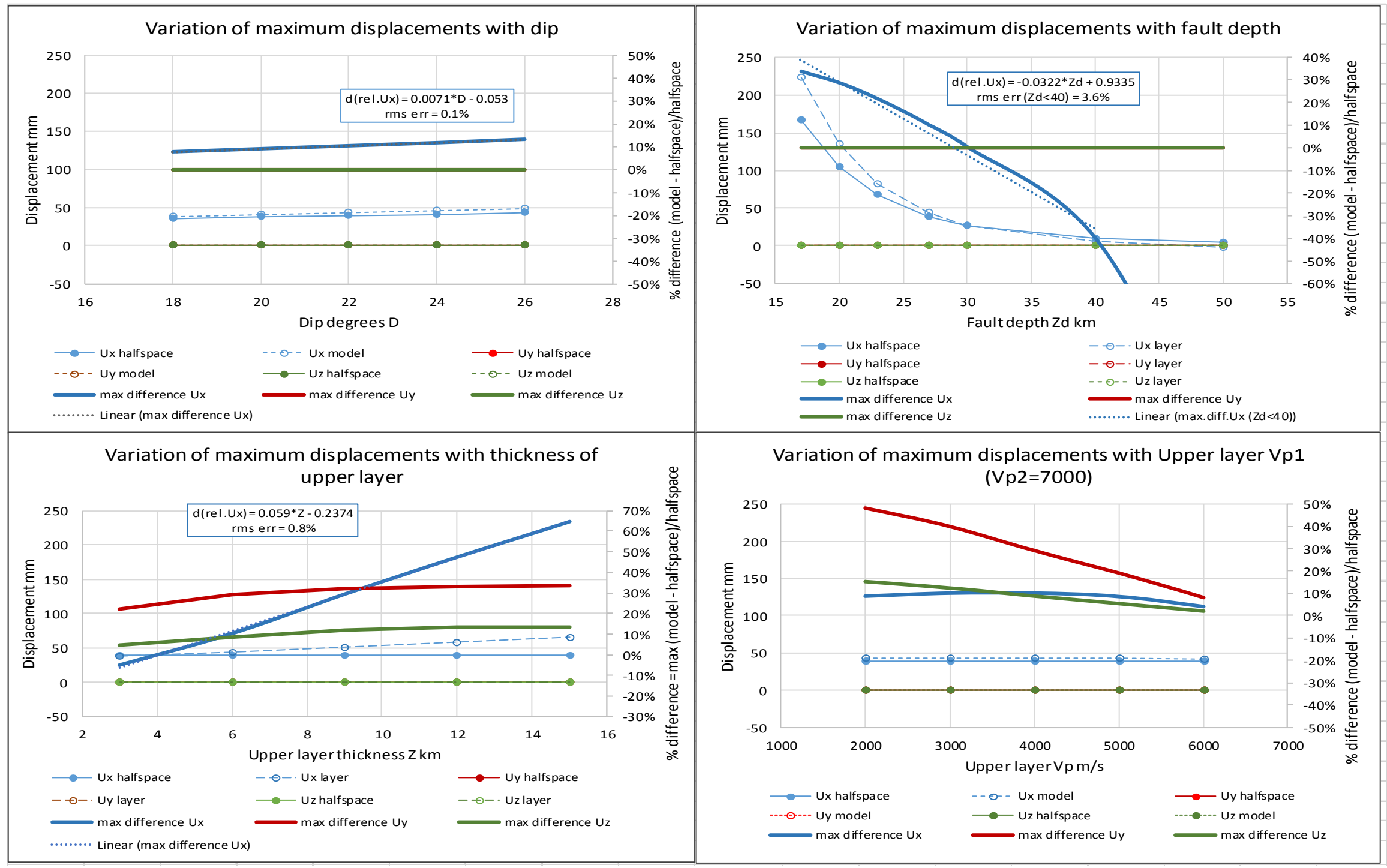


blank 
Figure A13e: Variation of surface displacements for a deep strike-slip fault with differing values of (in clockwise order from top left): dip (from $18^{\circ}$ to $26^{\circ}$ ); fault depth (from 17 to $50 \mathrm{~km}$ ); thickness of the upper layer (from 3 to $15 \mathrm{~km}$ ); and upper layer seismic velocity (from 2 to $6 \mathrm{~km} / \mathrm{s}$, corresponding to $\mathrm{E}$ ratios of 0.06, 0.15, 0.28, 0.46 and 0.71 when compared with plots in Cattin et al. [1999]). Solid lines with markers indicate maximum displacements from the Okada model, dashed lines indicate displacements from the layered model (both on the left scale), bold lines with no markers indicate percentage differences between the models (right scale), and dotted lines indicate linear regressions on the differences. Linear regression models with rms errors from linearity are shown in boxes, where relevant.

The results in Fig. A13e show that for the deep strike-slip scenario:

- Variations in dip angle have little significant effect ( 5\% in displacements along the strike direction) on the comparison between the Okada and layered models.

- Variations in fault depth give rise to significant differences in the along-strike displacements that are almost linear with respect to depth until approximately $40 \mathrm{~km}$ depth. At less than $30 \mathrm{~km}$ depth, the layered model shows less horizontal surface displacement than the Okada model, but the magnitudes of displacement are then very small.

- Variations in upper layer thickness give rise to differences in along-strike displacement that are very significant, but almost linear (from almost zero at $3 \mathrm{~km}$ thickness to $\sim 65 \%$ at $15 \mathrm{~km}$ thickness).

- Variations in elasticity ratio give rise to relatively small and reasonably constant alongstrike displacement differences. Whilst the proportional differences in the $\mathrm{Y}$ and $\mathrm{Z}$ directions appear to change significantly, the absolute magnitudes of these displacements are close to zero.

Variations in surface displacements and relative differences for the two models, as the rake is varied from pure thrust to strike-slip, are shown in Fig. A13f.

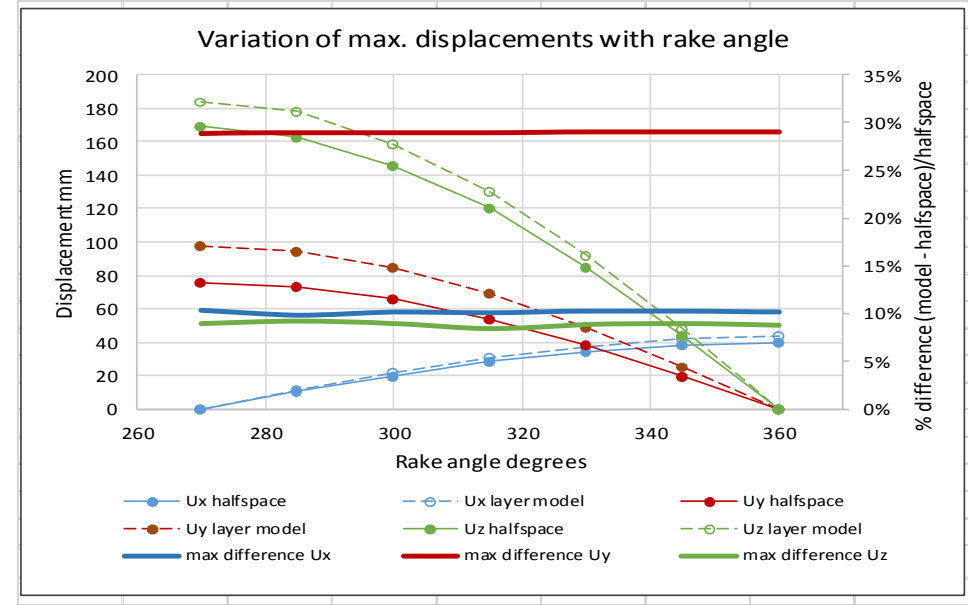

Figure A13f: Variations in surface displacements and relative differences between the Okada and layered models arising from change in rake from thrust to strike-slip. Whilst individual component displacements change significantly in magnitude as the rake is changed from pure thrust to pure strike-slip, the proportional differences between the models remain almost constant. 
As indicated in references noted in 2.6.4, softer strata above a half-space containing a fault are expected to produce larger surface displacements than an equivalent homogenous half-space model. However, it will be noted in some of the strike-slip models shown in Fig. A13f, that the layered model produced significantly smaller surface displacements than the Okada model in some cases. It was considered important to verify that this result is consistent with published information.

The ground displacement response from the 1997 strike-slip earthquake at Izmit, Turkey has been analysed by Hearn and Burgmann [2005] and $R$ Wang et al. [2003]. Wang's Fig. 3 suggests that surface displacements with the layered model would be less than with the homogenous half-space model. Hearn comments that with a layered model (and lower modulus in the upper layer) "forward-modeled surface displacements attenuate more dramatically with distance from the rupture than they do in a uniform half-space." This is the behaviour seen in plot 6 above, as the depth of the fault is increased - the initially greater surface displacements diminish more rapidly in the layered model, and beyond about $30 \mathrm{~km}$ depth, they become less than the displacements from the Okada model, hence moving the difference plot into negative space in the case of the strike-slip geometry (the same effect is seen in the thrust model plots, but the magnitude of the attenuation is less and the difference plots remains in positive space).

\section{Summary}

The effects can be summarised in terms of the proportionate difference in surface displacements between the layered model and the Okada model on a fault of fixed extent and with a fixed source displacement. It may be noted that varying the elastic parameters used in the Okada model has little effect within the normal range. 


\begin{tabular}{|c|c|c|}
\hline & \multicolumn{2}{|c|}{$\begin{array}{l}\text { Effect on ratio of maximum displacement from layered model to Okada } \\
\text { (homogenous) half-space model }\end{array}$} \\
\hline $\begin{array}{l}\text { Due to } \\
\text { changes in: }\end{array}$ & Thrust fault & Strike-slip fault \\
\hline Dip & Negligible $\left(10^{\circ}\right.$ to $\left.18^{\circ}\right)$ & Small effect $(\sim 5 \%)$ on $\mathrm{Ux}\left(18^{\circ}\right.$ to $\left.26^{\circ}\right)$ \\
\hline Fault depth & $\begin{array}{l}\text { Small effect on } \mathrm{Uz}(+10 \%) \text { as depth } \\
\text { increases from } 12 \text { to } 26 \mathrm{~km}\end{array}$ & $\begin{array}{l}\text { Significant negative effect }(-50 \%) \text { on Ux } \\
\text { as depth increases from } 17 \text { to } 40 \mathrm{~km}\end{array}$ \\
\hline $\begin{array}{l}\text { Upper layer } \\
\text { thickness }\end{array}$ & $\begin{array}{l}\text { Significant effect on } \mathrm{Uy}(+17 \%) \text { and } \\
\text { less on } \mathrm{Uz}(+7 \%) \text { with upper layer } \\
\text { thickness increasing from } 2 \text { to } 10 \mathrm{~km}\end{array}$ & $\begin{array}{l}\text { Significant effect on } \mathrm{Ux}(+70 \%) \text { as } \\
\text { upper layer thickness increases from } 3 \text { to } \\
15 \mathrm{~km})\end{array}$ \\
\hline $\begin{array}{l}\text { Modulus } \\
\text { ratio } \\
\text { (upper/lower) }\end{array}$ & $\begin{array}{l}\text { Significant effect on } \mathrm{Uy}(+40 \%) \text { and } \\
\mathrm{Uz}(+11 \%) \text { as modulus ratio } \\
\text { increases from } 1.4 \text { to } 16.7 \text { (= } \mathrm{Vp} \\
\text { reduction from } 86 \% \text { to } 29 \%)\end{array}$ & $\begin{array}{l}\text { Very small effect on } \mathrm{Ux}(\sim 5 \%) \text { for the } \\
\text { same change in modulus ratio. (Uy and } \\
\text { Uz displacements are zero) }\end{array}$ \\
\hline Source rake & \multicolumn{2}{|c|}{$\begin{array}{l}\text { No significant effect on the proportionate differences between the homogenous } \\
\text { and layered models as Rake changes from pure thrust }\left(270^{\circ}\right) \text { to pure strike slip } \\
\left(360^{\circ}\right) \text {. }\end{array}$} \\
\hline
\end{tabular}

Whilst the effect of varying the fault depth on the differences between the models is small and can probably be ignored in the thrust model, this is not the case in the strikeslip model. Whilst this apparent complication should be taken into account, in practice it may not be necessary, since the events in the south west appear to have taken place at similar depths, according to Wallace and Beavan [2010]. Furthermore, whilst linear approximations have been suggested for the relatively shallow depth ranges used in the calculations, it can be seen that the effects are curvilinear on the absolute magnitudes of displacement, and diminish significantly as the depths increase. The range of depths was extended to $50 \mathrm{~km}$ in the strike-slip case to examine this situation.

It can be seen in Fig. A13e that as the fault depth increases in the deep strike-slip scenario, the absolute ratio of the difference between the two models in Ux continues to increase, but the absolute values of the surface displacements become so small as render the apparent discrepancy of almost no practical effect within the range that we are considering. 
On the assumption that inversion calculations based on the Okada homogenous halfspace model will be primarily controlled by the maximum values of surface displacements at the various cGPS locations, the above observations lend themselves to the possibility of a first-order correction to the calculated source displacements if an appropriate simplified single layer model for each site can be developed. This is discussed in section 4.6. 


\section{Appendix 14 - Modeling 'negative' surface displacements (ref. 5.2.3)}

A. Subduction thrusts

Appendix 13 reports a study where parameters were varied one by one, and displacements calculated for a section perpendicular to strike at mid-length of the modeled source slip plane. Whilst this shows that 'negative' eastwards surface displacements could result for a strike-slip event at depth, no such 'negative' displacements are shown for a pure thrust fault. In order to explore whether more realistic geometries could give rise to 'negative' surface displacements, the model was extended to cover two dimensions on the surface, and the fault strike and thrust vectors adjusted to more closely represent situations pertaining in the northeast and southwest parts of the North Island. Estimates of fault strike were taken from Fig. 1 of Lamb and Smith [2013], and thrust direction was estimated from the mean SSE vectors shown in Fig. 3.3a of this thesis.

In the two cases, analyses were performed with and without layering using the EDGRN/EDCMP code. For the purposes of demonstration, in both cases, an upper layer of $4 \mathrm{~m} / \mathrm{s}$ velocity and $6 \mathrm{~km}$ thickness was used.

The figures illustrate displacements in the E direction over a 200 by $200 \mathrm{~km}$ surface area centred over the centre of the slip on the $30 \mathrm{~km}$ long by $20 \mathrm{~km}$ wide fault. The fault strikes, depths and dips, and thrust rakes are shown in the figures. In each case, the homogenous half-space model is shown first. In all cases the approximately triangular pale blue areas to the south represent areas with 'negative' surface displacements.

It can be seen that both geometries give rise to the possibility of areas of 'negative' surface E-component displacement, and that imposing a layered structure, whilst changing the magnitude of the positive displacement, has only a small effect on the magnitude and extent of the 'negative' displacements. 


\section{Northeast:}

Okada option, E displacements from fault strike 025, depth $30 \mathrm{~km}$, dip - 10 , source rake 280

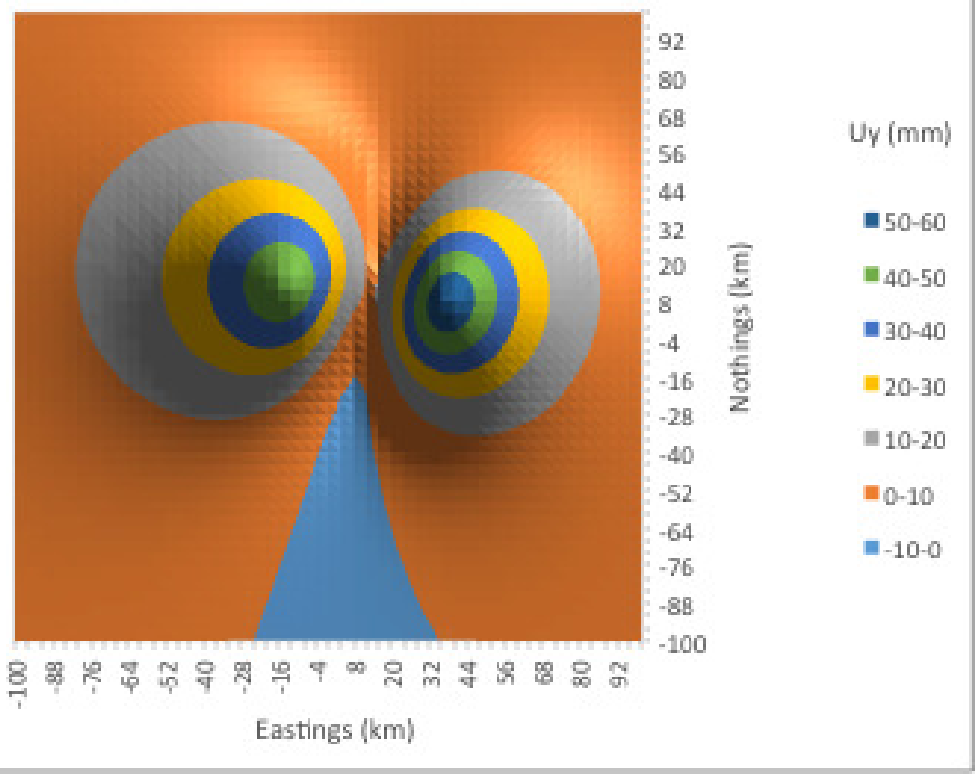

Layered option, E displacements from fault strike 025 , depth $30 \mathrm{~km}$, source rake 280

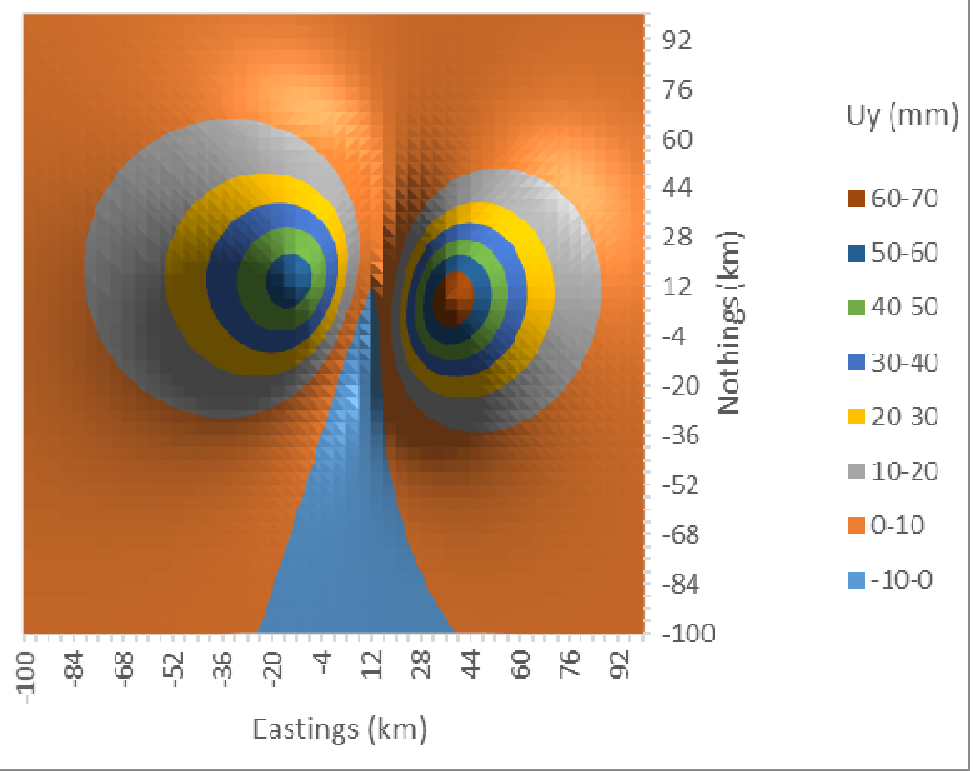




\section{Southwest:}

Okada option, E displacements from fault strike 075 , depth $45 \mathrm{~km}$, dip -22 , source rake 345

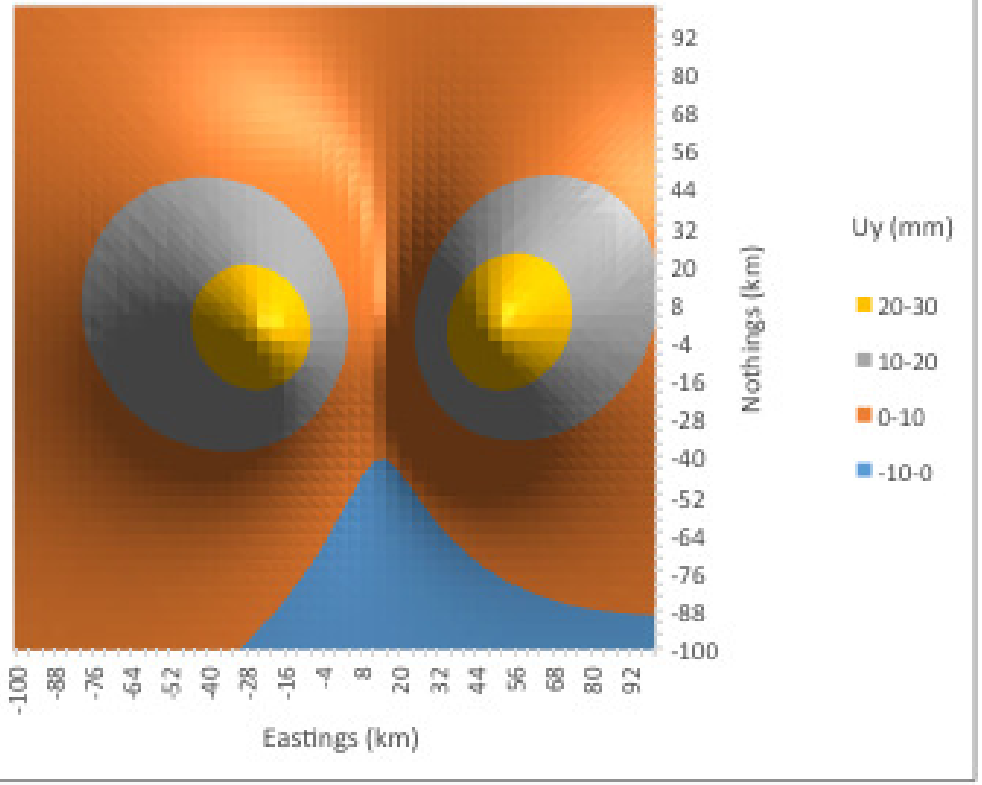

Layered option, E displacements from fault strike 075 , depth $45 \mathrm{~km}$, dip -22, source rake 345

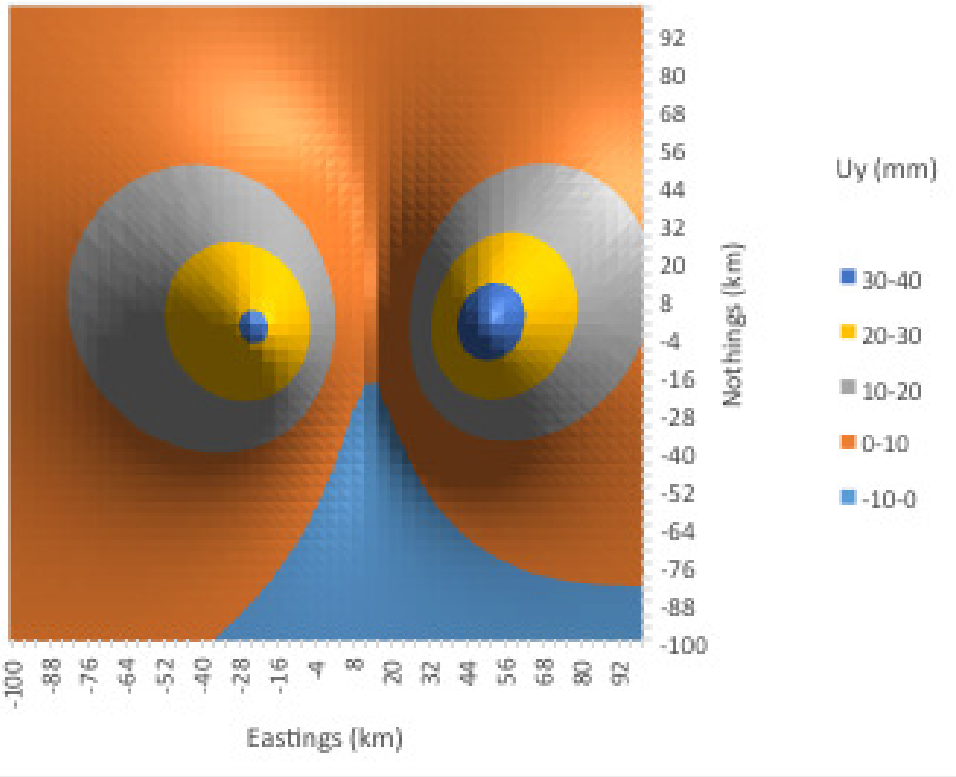




\section{B. Imbricate faults and back-thrusts}

In order to establish whether imbricate faults and backthrusts, which, from seismic sections, appear relatively common along and offshore the East coast, modeling was carried out using the Okada code. A small $(5 \times 5.7 \mathrm{~km})$, buried (15 km depth) fault, striking 000 and subjected to thrust with $1 \mathrm{~m}$ slip and rake 270 was modeled at different dip angles. The surface displacements in all 3 components over an area of $100 \mathrm{~km}$ square were calculated, but for the purposes of illustration, the $\mathrm{E}$ component displacements across the middle of the fault are shown below. These suggest that for imbricate faults with dip exceeding $60^{\circ}$, or for backthrusts with dip exceeding $40^{\circ}, \mathrm{E}$ component surface displacements would appear 'negative' (i.e. trending west):
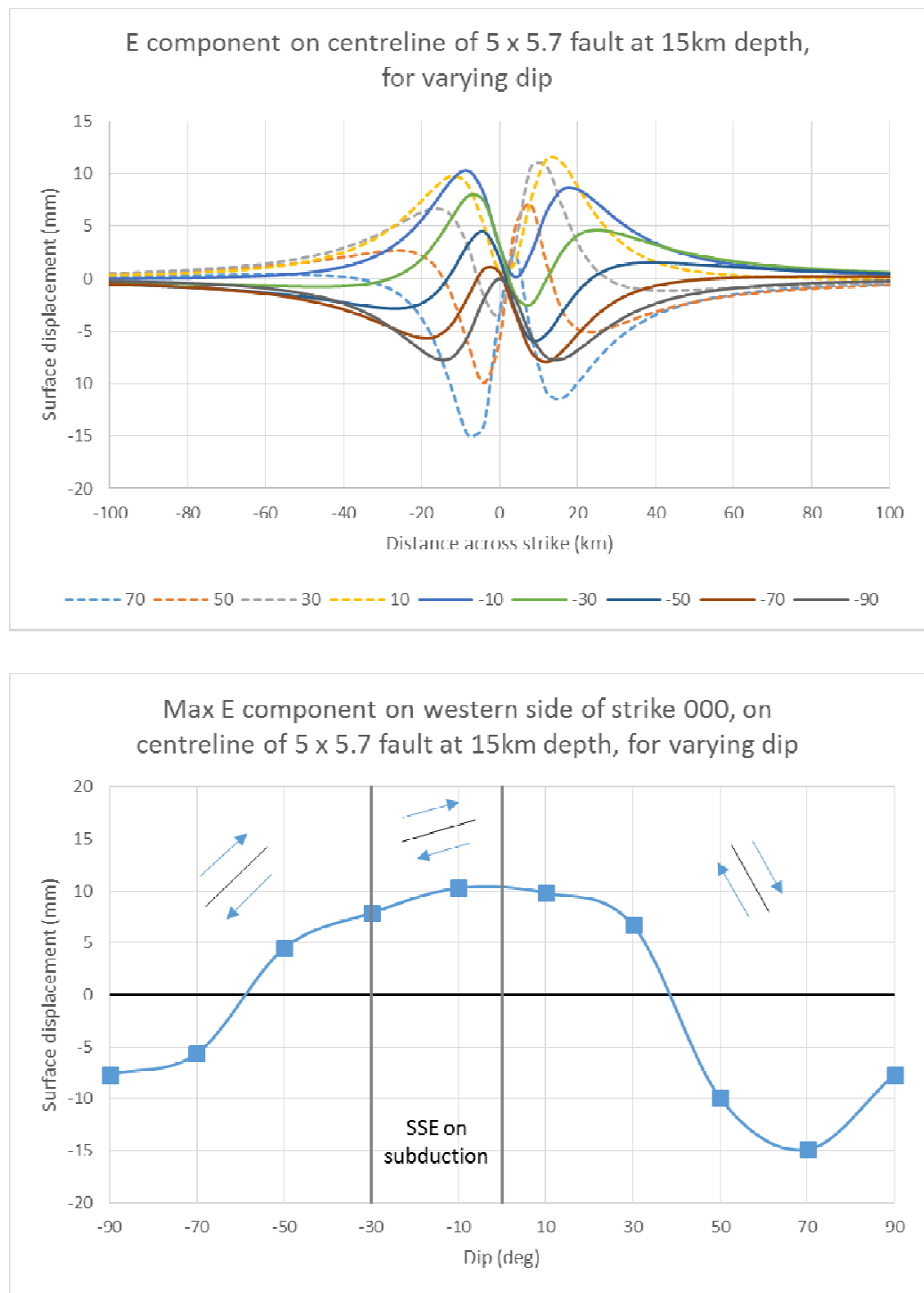


\section{Appendix 15 - Comparison of inversion results with Table 2 of Wallace and Beavan (2010)}

(ref 5.4.1)

\begin{tabular}{|c|c|c|c|c|c|c|c|c|c|c|c|c|c|c|c|c|c|c|c|c|c|}
\hline \multicolumn{12}{|c|}{ Wallace \& Beavan Table 2} & \multicolumn{10}{|c|}{ This project } \\
\hline & \multicolumn{3}{|c|}{ Event } & $\begin{array}{c}\text { Avg.Slip } \\
\text { (cm) }\end{array}$ & $\begin{array}{c}\text { Area } \\
(\mathrm{km} 2)\end{array}$ & $\begin{array}{c}\text { Moment } \\
(\mathrm{Nm})\end{array}$ & \multirow{2}{*}{$\begin{array}{c}\text { Moment } \\
(\mathrm{Nm})\end{array}$} & \multirow[t]{2}{*}{ Mw } & \multirow{2}{*}{$\mid \begin{array}{c}\text { Duration } \\
\text { (days) }\end{array}$} & \multirow{2}{*}{\multicolumn{2}{|c|}{$\begin{array}{l}\text { Est. main centre } \\
\text { Lat | Long }\end{array}$}} & \multirow{3}{*}{$\begin{array}{l}\text { Event } \\
\text { no. }\end{array}$} & \multicolumn{2}{|c|}{ Slip } & \multirow{3}{*}{\begin{tabular}{c|} 
Area \\
$(\mathrm{km} 2)$
\end{tabular}} & \multirow{3}{*}{$\begin{array}{c}\text { Moment } \\
(\mathrm{Nm})\end{array}$} & \multirow{3}{*}{$\begin{array}{l}95 \% \mathrm{Cl} \\
(\mathrm{Nm})\end{array}$} & \multirow[t]{3}{*}{ Mw } & \multirow{3}{*}{\begin{tabular}{|c|} 
Duration \\
(days)
\end{tabular}} & \multirow[t]{3}{*}{ Lat } & \multirow[t]{3}{*}{ Long } \\
\hline \multirow{2}{*}{\multicolumn{12}{|c|}{ 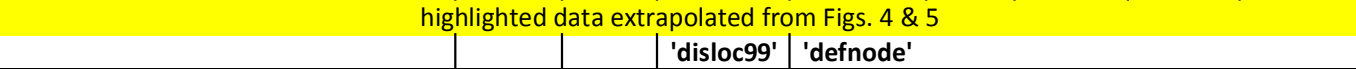 }} & & & (cm) & & & & & & & \\
\hline & & & & & & & & & & & & & & & & & & & & & \\
\hline \multicolumn{12}{|l|}{ Southern } & & & & & & & & & & \\
\hline Kapiti & Nov & 2003 & & 15.7 & 1704 & $1.07 E+19$ & $1.13 \mathrm{E}+19$ & 6.6 & 202 & -40.9 & 174.5 & 3 & 10 & 6 & 1356 & $6.49 \mathrm{E}+18$ & $1.60 E+18$ & 6.5 & 198 & -41.1 & 174.6 \\
\hline Manawatu & Feb & 2004 & (stage 1) & 9.2 & 6390 & $2.35 \mathrm{E}+19$ & $2.74 E+19$ & 6.9 & 323 & -39.9 & 175.6 & 6 & 12 & 2 & 3386 & $1.64 \mathrm{E}+19$ & $7.06 \mathrm{E}+18$ & 6.7 & 295 & -40.2 & 175.3 \\
\hline Manawatu & Jan & 2005 & (stage 2) & 24.4 & 2309 & $2.25 E+19$ & $2.49 E+19$ & 6.9 & 87 & -39.9 & 175.6 & 16 & 31 & 2 & 2159 & $2.67 \mathrm{E}+19$ & $7.88 \mathrm{E}+18$ & 6.9 & 217 & -40.0 & 175.9 \\
\hline Manawatu & Mar & 2005 & (stage 3) & 23.3 & 2127 & $1.98 \mathrm{E}+19$ & $1.99 E+19$ & 6.8 & 83 & -40.2 & 175.4 & & & & & & & & & & \\
\hline Manawatu & & 2004 & total & & & $6.58 E+19$ & $7.19 E+19$ & 7.2 & 492 & & & & & & & & & & & & \\
\hline Kapiti & Sep & 2007 & (stage 1) & 5.8 & 1271 & $2.94 \mathrm{E}+18$ & $3.35 E+18$ & 6.3 & 80 & -40.8 & 174.5 & & & & & & & & & & \\
\hline Kapiti & Jan & 2008 & (stage 2) & 9.7 & 4075 & $1.58 \mathrm{E}+19$ & $1.65 E+19$ & 6.7 & 100 & -40.8 & 174.4 & & & & & & & & & & \\
\hline Kapiti & Mar & 2008 & (stage 3) & 2.6 & 9163 & $9.55 E+18$ & $9.64 E+18$ & 6.6 & 110 & -41.3 & 174.4 & & & & & & & & & & \\
\hline Kapiti & Jul & 2008 & (stage 4) & 2.9 & 6706 & $7.67 \mathrm{E}+18$ & $5.90 E+18$ & 6.5 & 190 & -41.2 & 174.4 & & & & & & & & & & \\
\hline Kapiti & & 2008 & total & & & $3.59 \mathrm{E}+19$ & $3.53 \mathrm{E}+19$ & 7 & 479 & & & 41 & 26 & 6 & 3497 & $3.75 \mathrm{E}+19$ & $6.55 \mathrm{E}+18$ & 7 & 419 & -41.0 & 174.3 \\
\hline \multicolumn{4}{|l|}{ Northern } & & & & & & & & & & & & & & & & & & \\
\hline Gisborne & Oct & 2002 & & 10.2 & 2169 & $8.81 \mathrm{E}+18$ & $1.81 \mathrm{E}+19$ & 6.8 & 14 & -38.9 & 178.1 & 2 & 11 & -5 & 1261 & $6.14 \mathrm{E}+18$ & $1.27 \mathrm{E}+17$ & 6.5 & 56 & -38.6 & 178.0 \\
\hline Gisborne & Nov & 2004 & & 12 & 1047 & $5.02 \mathrm{E}+18$ & $1.21 \mathrm{E}+19$ & 6.7 & 17 & -38.8 & 178.2 & 14 & 27 & 1 & 635 & $7.26 \mathrm{E}+18$ & $4.38 \mathrm{E}+18$ & 6.5 & 40 & -38.8 & 178.2 \\
\hline Tolaga Bay & Dec & 2004 & & 1.5 & 1667 & $9.71 \mathrm{E}+17$ & $4.01 \mathrm{E}+18$ & 6.3 & 20 & -38.2 & 178.7 & 15 & 4 & 0 & 2059 & $4.28 \mathrm{E}+18$ & $4.59 E+18$ & 6.4 & 37 & -38.3 & 178.6 \\
\hline S.Hawkes Bay & Jun & 2006 & & 11.1 & 908 & $4.04 \mathrm{E}+18$ & $5.29 \mathrm{E}+18$ & 6.4 & 7 & -40.3 & 177.3 & 25 & 7 & 1 & 1243 & $3.58 \mathrm{E}+18$ & $6.23 E+17$ & 6.3 & 46 & -39.9 & 177.2 \\
\hline Gisborne & Jul & 2006 & & 2.9 & 4903 & $5.64 \mathrm{E}+18$ & $1.15 \mathrm{E}+19$ & 6.6 & 6 & -38.8 & 178.3 & 26 & 13 & 4 & 1241 & $6.83 \mathrm{E}+18$ & $1.73 E+18$ & 6.5 & 23 & -38.9 & 178.2 \\
\hline S.Hawkes Bay & Aug & 2006 & & 14.7 & 3251 & $1.91 \mathrm{E}+19$ & $1.78 \mathrm{E}+19$ & 6.8 & 7 & -40.4 & 177.3 & 29 & 20 & -8 & 3042 & $2.71 \mathrm{E}+19$ & $3.51 \mathrm{E}+18$ & 6.9 & 50 & -40.4 & 177.3 \\
\hline N.of Gisborne & Dec & 2007 & & 7 & 679 & $1.90 \mathrm{E}+18$ & $6.72 \mathrm{E}+18$ & 6.5 & 34 & -38.5 & 178.6 & 44 & 15 & 6 & 2041 & $1.37 \mathrm{E}+19$ & $4.89 \mathrm{E}+18$ & 6.7 & 50 & -38.8 & 178.5 \\
\hline S.Hawkes Bay & Feb & 2008 & & 3.8 & 1958 & $2.95 \mathrm{E}+18$ & $4.75 E+18$ & 6.4 & 5 & -40.2 & 177.3 & 46 & 3 & -3 & 1241 & $2.10 \mathrm{E}+18$ & $4.01 \mathrm{E}+17$ & 6.1 & 26 & -40.0 & 177.2 \\
\hline Mahia & Mar & 2008 & & 5 & 6005 & $1.20 \mathrm{E}+19$ & $1.30 \mathrm{E}+19$ & 6.7 & 15 & -39.3 & 177.9 & 50 & 5 & -1 & 2061 & $4.35 \mathrm{E}+18$ & $6.25 \mathrm{E}+17$ & 6.4 & 37 & -39.1 & 177.8 \\
\hline Tolaga Bay & Aug & 2008 & & 5.1 & 1028 & $2.10 E+18$ & $4.99 \mathrm{E}+18$ & 6.4 & 12 & -38.5 & 178.7 & 59 & 4 & -3 & 2061 & $4.12 \mathrm{E}+18$ & $1.68 \mathrm{E}+18$ & 6.3 & 42 & -38.4 & 178.5 \\
\hline $\begin{array}{l}\text { Tolaga Bay } \\
\text { Mahia }\end{array}$ & Feb & 2010 & & $\begin{array}{c}6.5 \\
3\end{array}$ & 6897 & $9.66 \mathrm{E}+18$ & $1.52 E+19$ & 6.7 & 14 & $\begin{array}{l}-38.5 \\
-39.3\end{array}$ & $\begin{array}{l}178.6 \\
177.8\end{array}$ & 81 & 20 & -2 & 3048 & $2.47 \mathrm{E}+19$ & $4.82 E+18$ & 6.9 & 59 & -39.5 & 177.9 \\
\hline Gisborne & Mar & 2010 & & 11.5 & 3146 & $1.44 \mathrm{E}+19$ & $1.65 E+19$ & 6.7 & 16 & -39.0 & 178.2 & 90 & 7 & -4 & 1230 & $6.26 \mathrm{E}+18$ & $5.46 \mathrm{E}+18$ & 6.5 & 52 & -39.2 & 178.4 \\
\hline
\end{tabular}




\section{Appendix 16 - Comparison of displacements at sites WGTN and WGTT}

As discussed in section 5.5, WGTN and WGTT are located within $5 \mathrm{~km}$ of each other. 10 events were detected at WGTN and 14 at WGTT during the 14-year period covered by this report. The individual amplitudes are shown plotted below for each component, together with the larger of the rms errors for the two sites. Events 144 and 145 relate to the 2013 Cook Strait and Seddon earthquakes. It should also be noted that events are 'picked' using the $\mathrm{E}$ component, and displacements for $\mathrm{N}$ and $\mathrm{U}$ components are automatically calculated from the picked start and end times.
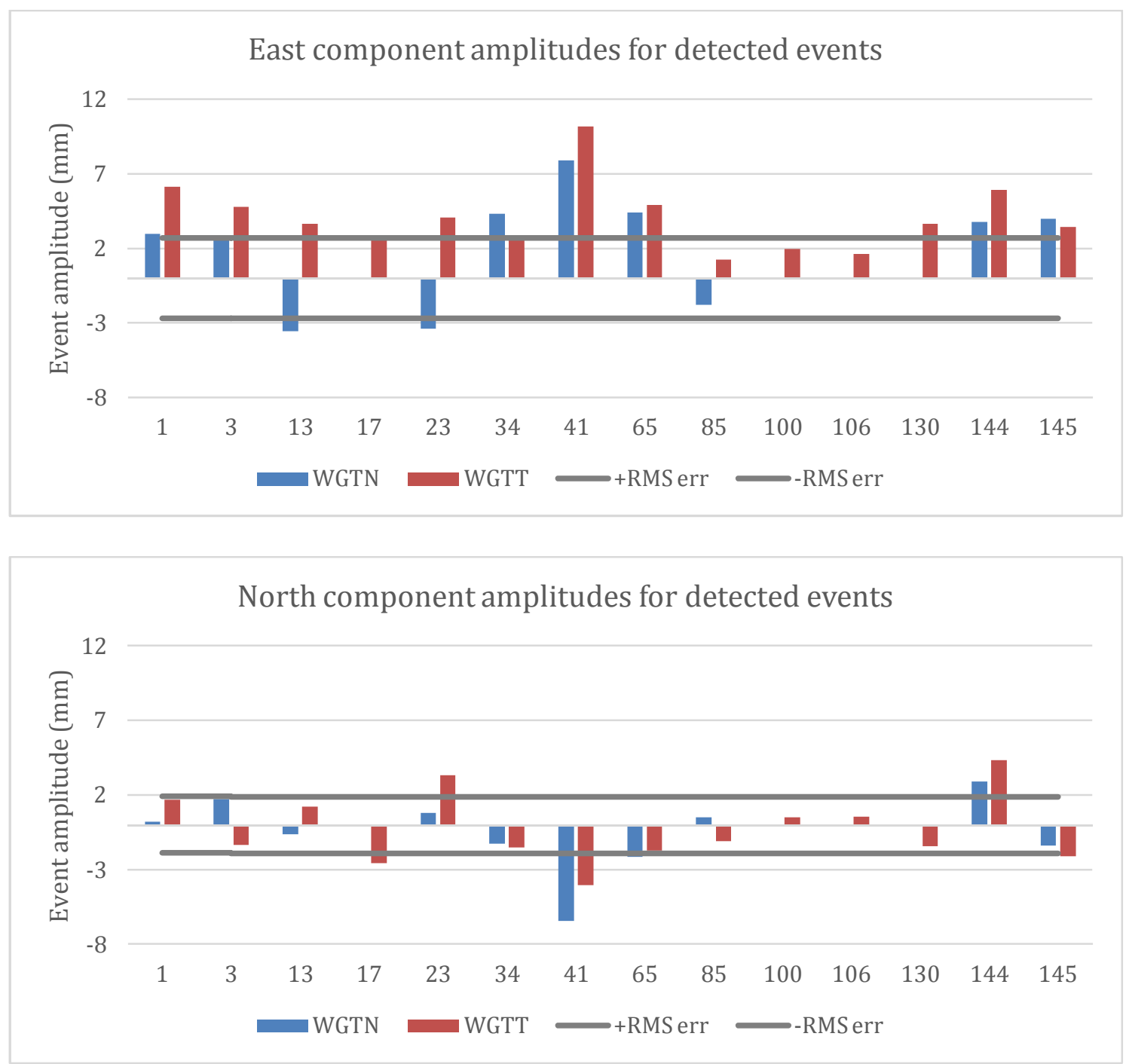


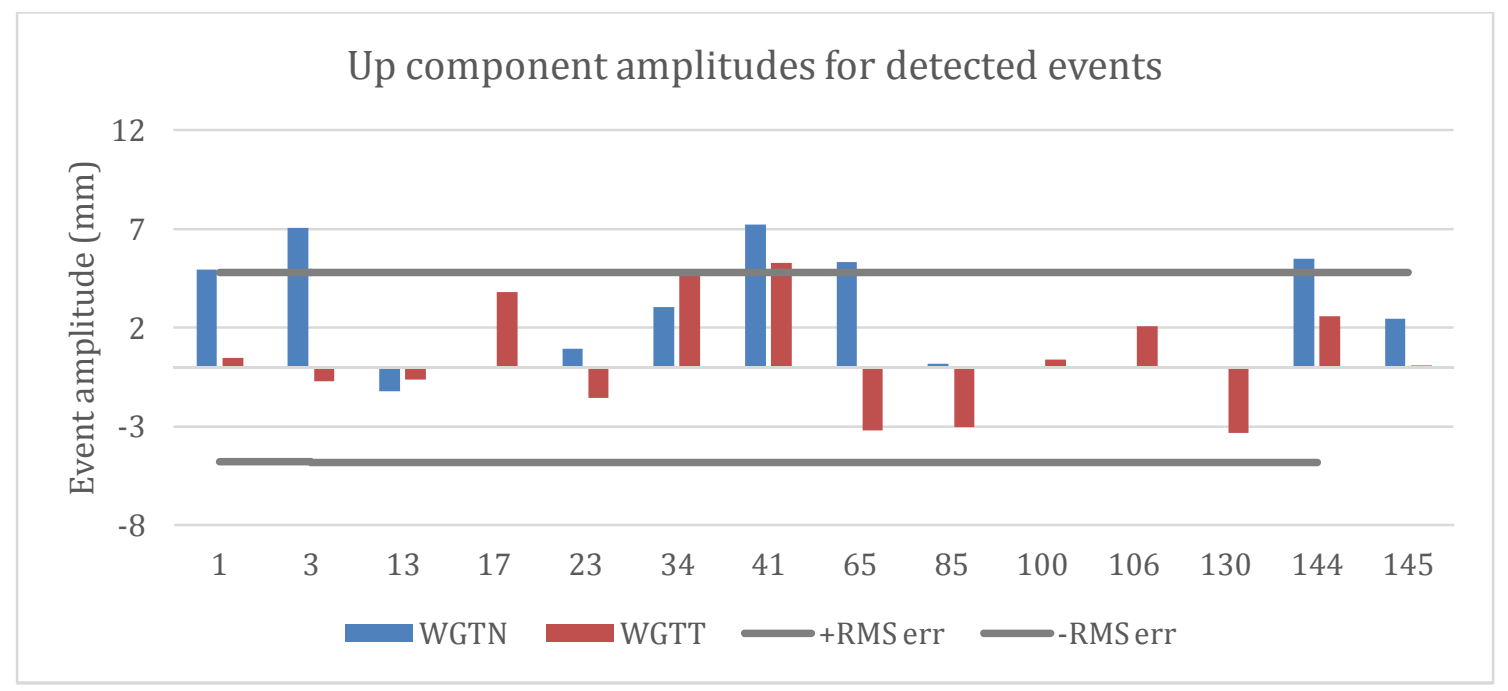

If all results are considered equally valid, then due to SSEs over the 14 years, WGTN moved a total of $39 \mathrm{~mm} \mathrm{E,} 18 \mathrm{~mm} \mathrm{~N}$ and $38 \mathrm{~mm} \mathrm{U}$ compared with $57 \mathrm{~mm} \mathrm{E,} 27 \mathrm{~mm} \mathrm{~N}$ and $32 \mathrm{~mm} \mathrm{U}$ at WGTT; or, in terms of paired differences for the 10 events identified in common, the average differences WGTT-WGTN were $21(+/-45) \%$ E, $25(+/-291) \%$ $\mathrm{N}$, and $-246(+/-508) \% \mathrm{U}$. The average paired differences for the $\mathrm{E}$ and $\mathrm{U}$ directions are significantly different to zero on a 1 -sided t-test at only the $8 \%$ level (whereas $5 \%$ is usually taken, somewhat arbitrarily, as the criterion for significance).

Several of these individual displacements are at or below what may be considered the noise threshold (+/- $1 \mathrm{rms}$ error) for the E component, and most are at or below the thresholds for $\mathrm{N}$ and $\mathrm{U}$ components. It can be seen that in some cases one site registered a displacement in the opposite direction to the other, and given that all source events are assumed to have taken place in the far field, it seems improbable that such individual comparisons are valid. It was therefore considered potentially inappropriate to assess the comparative statistics using all 10 individual paired differences.

For the 7 comparisons for the E component where the SSE displacements exceed the rms error level, and are in the same direction (i.e. events 1, 3, 34, 41, 65, 144 and 145), the WGTT amplitude exceeds the WGTN amplitude by an average of $31 \%$ (sd 50\%), which is statistically significantly different to zero only at the $7 \%$ level. 